\title{
Timing of Introduction of Complementary Foods and Beverages and Growth, Size, and Body Composition: A Systematic Review
}

The Pregnancy and Birth to 24 Months Project

Published date: April 15 2019

Nutrition Evidence Systematic Review

Center for Nutrition Policy and Promotion

Food and Nutrition Service

U.S. Department of Agriculture

3101 Park Center Drive

Alexandria, Virginia 
This systematic review was conducted for the Pregnancy and Birth to 24 Months Project (P/B-24 Project) by the Nutrition Evidence Systematic Review (NESR) team at the Center for Nutrition Policy and Promotion, Food and Nutrition Service, USDA. All systematic reviews from the P/B-24 Project are available on the NESR website: https://nesr.usda.gov.

Conclusion statements drawn as part of this systematic review describes the state of science related to the specific question examined. Conclusion statements do not draw implications, nor should they be interpreted to be dietary guidance.

The contents of this document may be used and reprinted without permission. Endorsement by NESR, the Center for Nutrition Policy and Promotion, the Food and Nutrition Service, or the U.S. Department of Agriculture of derivative products developed from this work may not be stated or implied.

In accordance with Federal civil rights law and U.S. Department of Agriculture (USDA) civil rights regulations and policies, the USDA, its Agencies, offices, and employees, and institutions participating in or administering USDA programs are prohibited from discriminating based on race, color, national origin, religion, sex, gender identity (including gender expression), sexual orientation, disability, age, marital status, family/parental status, income derived from a public assistance program, political beliefs, or reprisal or retaliation for prior civil rights activity, in any program or activity conducted or funded by USDA (not all bases apply to all programs). Remedies and complaint filing deadlines vary by program or incident.

Persons with disabilities who require alternative means of communication for program information (e.g., Braille, large print, audiotape, American Sign Language, etc.) should contact the responsible Agency or USDA's TARGET Center at (202) 720-2600 (voice and TTY) or contact USDA through the Federal Relay Service at (800) 877-8339. Additionally, program information may be made available in languages other than English.

To file a program discrimination complaint, complete the USDA Program Discrimination Complaint Form, AD3027, found online at How to File a Program Discrimination Complaint and at any USDA office or write a letter addressed to USDA and provide in the letter all of the information requested in the form. To request a copy of the complaint form, call (866) 632-9992. Submit your completed form or letter to USDA by: (1) mail:

U.S. Department of Agriculture, Office of the Assistant Secretary for Civil Rights, 1400 Independence Avenue, SW, Washington, D.C. 20250-9410; (2) fax: (202) 690-7442; or (3) email: program.intake@usda.gov.

USDA is an equal opportunity provider, employer, and lender.

Suggested citation for this systematic review: Nutrition Evidence Systematic Review Team and Complementary Feeding Technical Expert Collaborative. Timing of Introduction of Complementary Foods and Beverages and Growth, Size, and Body Composition: A Systematic Review. Pregnancy and Birth to 24 Months Project. Alexandria, VA: U.S. Department of Agriculture, Food and Nutrition Service, Center for Nutrition Policy and Promotion, February 2019. Available at: https://nesr.usda.gov/project-specific-overviewpb-24-0

This systematic review has also been published in the American Journal of Clinical Nutrition: English L, Obbagy JE, Wong YP, Butte NF, Dewey KG, Fox MK, Greer FR, Krebs NF, Scanlon KS, Stoody EE. Timing of introduction of complementary foods and beverages and growth, size, and body composition: a systematic review. Am J Clin Nutr. 2019: 109(7): 935S-55S doi: 10.1093/ajcn/nqy267

\section{Related citations:}

- P/B-24 Project overview: Stoody EE, Spahn JM, Casavale KO. The Pregnancy and Birth to 24 Months Project: a series of systematic reviews on diet and health. Am J Clin Nutr. 2019;109(7):685S-697S. doi: 10.1093/ajcn/ngy372

- P/B-24 systematic review methodology: Obbagy JE, Spahn JS, Psota TL, Spill MK, Dreibelbis C, Gungor DE, Nadaud PN, Raghavan R, Callahan EH, English LK, Kingshipp BJ, Lapergola CC, Shapiro MJ, Stoody EE. Systematic review methodology used in the Pregnancy and Birth to 24 Months Project. Am J Clin Nutr 2019;109(7):698S-704S. doi: 10.1093/ajcn/nqy226

- Related systematic reviews from the P/B-24 Project: English LK, Obbagy JO, Wong YP, Butte 
NF, Dewey KG, Fox MK, Greer FR, Krebs NK, Scanlon KS, Stoody EE. Types and amounts of complementary foods and beverages consumed and growth, size, and body composition: a systematic review. Am J Clin Nutr. 2019;109(7):956S-77S. doi: 10.1093/ajcn/nqy281. 


\section{ACKNOWLEDGEMENTS}

\section{Complementary Feeding Technical Expert Collaborative (TEC) members:}

- Nancy F. Butte, PhD, RD, USDA/ARS Children's Nutrition Research Center, Baylor College of Medicine, Department of Pediatrics, Emeritus

- Kathryn G. Dewey, PhD, University of California, Davis, Department of Nutrition

- David M. Fleischer, MD, Children's Hospital Colorado, University of Colorado School of Medicine, Department of Pediatrics, Section of Allergy and Immunology

- Mary Kay Fox, Med, Mathematic Policy Research

- Frank R. Greer, MD, University of Wisconsin School of Medicine and Public Health, Department of Pediatrics, Emeritus

- Nancy F. Krebs, MD, MS, University of Colorado School of Medicine, Department of Pediatrics

- Kelley S. Scanlon, PhD, RD, USDA Food and Nutrition Service; formerly of the Centers for Disease Control and Prevention, Division of Nutrition, Physical Activity, and Obesity

\section{Nutrition Evidence Systematic Review (NESR) team:}

- Laural K. Englishi, PhD, Panum Group, Lead Analyst (11/2016-project completion)

- Julie E. Obbagy, PhD, RD, USDA, Analyst (05/2016-project completion)

- Yat Ping Wong, MLS, MPH, USDA, Librarian

- Tricia Psota, PhD, USDA, Analyst (07/2015-06/2016)

- Perrine Naudadi, PhD, Panum Group, Analyst (07/2015-05/2016)

- Kirsten Johnsi, MS, Panum Group, Analyst (07/2015-05/2016)

- Nancy Terry, MLS, NIH, Librarian

\section{Project Leads:}

- Eve Essery Stoody, PhD, USDA

\section{Federal Expert Group (FEG)-Technical Expert Collaborative (TEC) Liaisons:}

- Kelley Scanlon, PhD RD, USDA Food and Nutrition Service; formerly of the Centers for Disease Control and Prevention, Division of Nutrition, Physical Activity, and Obesity

All TEC and NESR team members, Project leads, and FEG-TEC liaisons participated in establishing the research questions, analytic framework, and study inclusion and exclusion criteria. LKE, JEO, YWP, TLP, PN, KJ, and NT developed and conducted the literature search, screened search results, and identified studies for inclusion. LKE and JEO extracted data and assessed risk of bias for included studies. NFC, KGD, DMF, MKF, FRG, NFK, and KSS reviewed and provided substantive feedback on all systematic review materials, including the synthesis of the body of evidence, conclusion statement, and grade of the strength of the evidence. LKE prepared this

i Under contract with the Food and Nutrition Service, United States Department of Agriculture. 
report and EES provided oversight. All authors critically reviewed and approved the final report. The authors declare no conflicts of interest.

FUNDING SOURCE: United States Department of Agriculture, Food and Nutrition Service, Center for Nutrition Policy and Promotion, Alexandria, VA 


\section{TABLE OF CONTENTS}

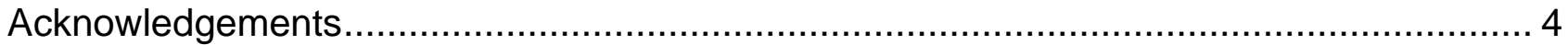

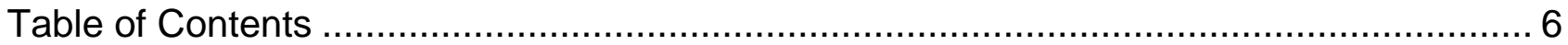

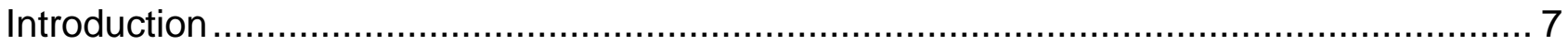

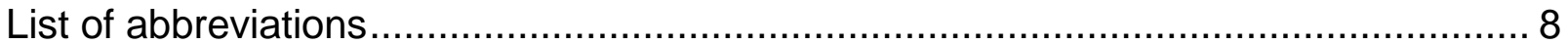

What is the relationship between the timing of introduction of complementary foods and beverages and growth, size, and body composition? .................................................... 9

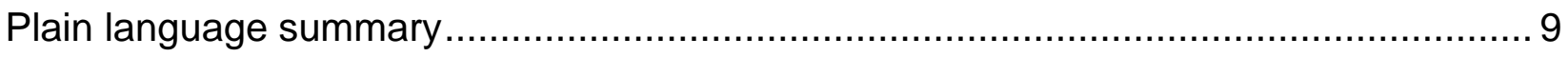

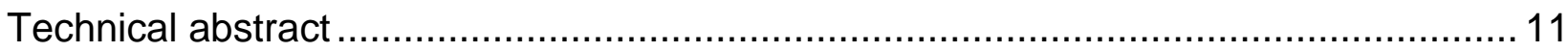

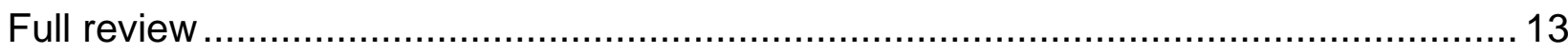

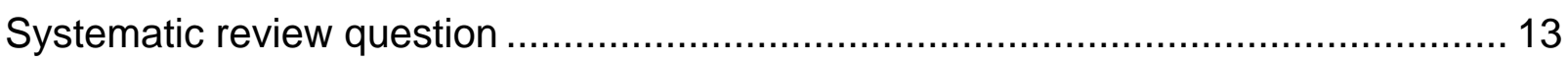

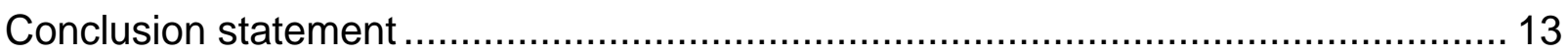

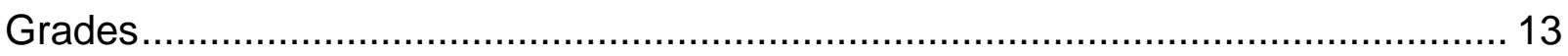

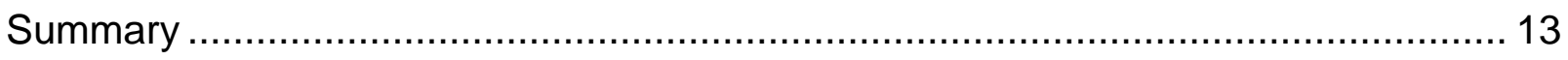

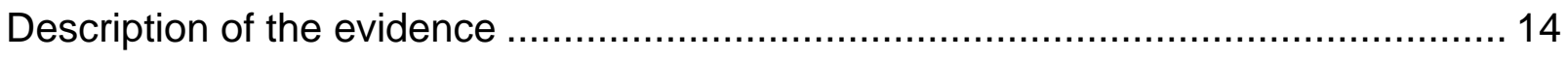

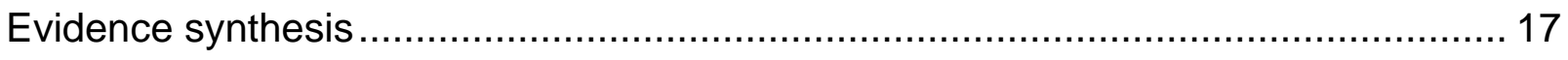

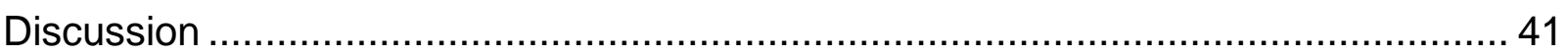

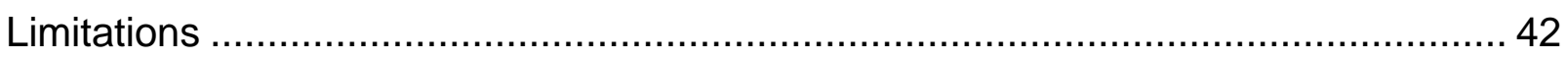

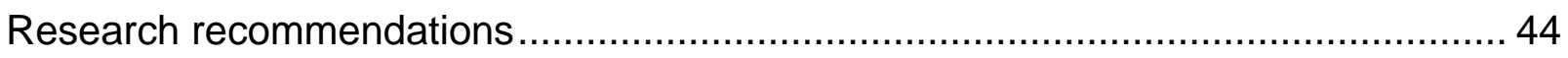

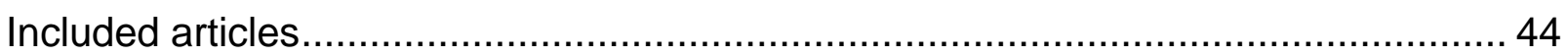

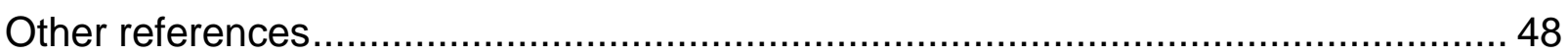

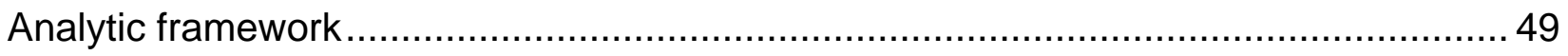

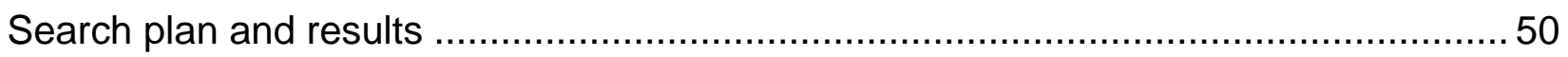

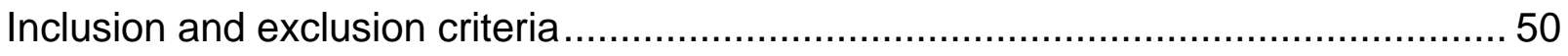

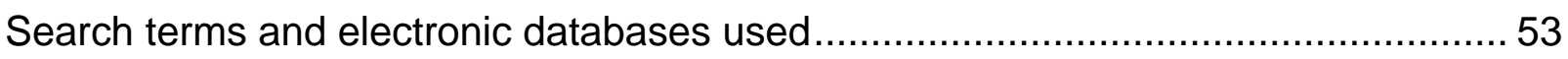

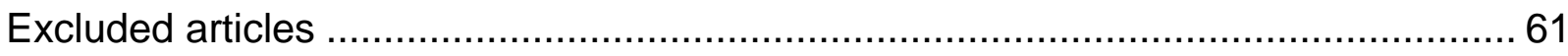

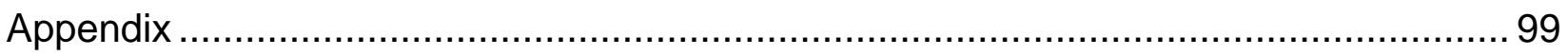

Table 1. Studies that examined the timing of introduction of complementary oods and beverages and growth, size, and body composition ...................................................... 23

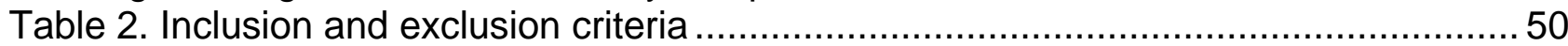

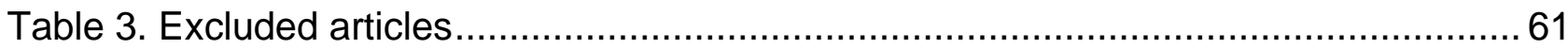

Supplemental Table S1. Description of the evidence examining timing of introduction of CFB and growth, size, and body composition............................................................ 99

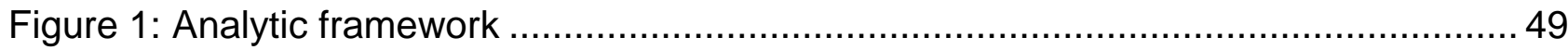

Figure 2: Flow chart of literature search and screening results....................................6 60 


\section{INTRODUCTION}

This document describes a systematic review conducted to answer the following question: What is the relationship between timing of introduction of complementary foods and beverages and growth, size, and body composition?. This systematic review was conducted as part of the Pregnancy and Birth to 24 Months Project (P/B-24 Project) by USDA's Nutrition Evidence Systematic Review (NESR).

The purpose of the P/B-24 Project was to conduct a series of systematic reviews on diet and health for women who are pregnant and for infants and toddlers from birth to 24 months of age. This project was a joint initiative led by USDA and HHS, and USDA's NESR carried out all of the systematic reviews. A Federal Expert Group (FEG), a broadly representative group of Federal researchers and program leaders, also provided input throughout the P/B-24 Project. More information about the P/B-24 Project has been publishedii and is available on the NESR website: https://nesr.usda.gov/project-specificoverview-pb-24-0

NESR, formerly the Nutrition Evidence Library (NEL), specializes in conducting food- and nutrition-related systematic reviews using a rigorous, protocol-driven methodology. To conduct each P/B-24 systematic review, NESR's staff worked with a Technical Expert Collaborative (TEC), which is a group of 7-8 leading subject matter experts.

NESR's systematic review methodology involves developing and prioritizing systematic review questions, searching for and selecting studies, extracting and assessing the risk of bias of data from each included study, synthesizing the evidence, developing a conclusion statement, grading the evidence underlying the conclusion statement, and recommending future research. A detailed description of the methodology used in conducting systematic reviews for the P/B-24 Project has been publishediii and is available on the NESR website: https://nesr.usda.gov/pb-24-project-methodology-0. In addition, starting on page 49, this document includes details about the methodology as it was applied to the systematic review described herein. An analytic framework that illustrates the overall scope of the question, including the population, the interventions and/or exposures, comparators, and outcomes of interest, is found on page 49. In addition, the literature search plan that was used to identify studies included in this systematic review is found on page 50.

\footnotetext{
ii Stoody EE, Spahn JM, Cassavale KO. The Pregnancy and Birth to 24 Months Project: a series of systematic reviews on diet and health. Am J Clin Nutr. 2019;109(7):685S-697S. doi:10.1093/ajcn/ngy372

iii Obbagy JE, Spahn JS, Psota TL, Spill MK, Dreibelbis C, Gungor DE, Nadaud PN, Raghavan R, Callahan EH, English LK, Kingshipp BJ, Lapergola CC, Shapiro MJ, Stoody EE. Systematic review methodology used in the Pregnancy and Birth to 24 Months Project. Am J Clin Nutr. 2019;109(7):698S704S. doi: 10.1093/ajen/nqy226
} 


\section{List of abbreviations}

\begin{tabular}{ll}
\hline Abbreviation & Full name \\
\hline BF & Breast fed \\
\hline BMIZ & Body mass index z-score \\
\hline CF & Complementary feeding \\
\hline CFB & Complementary food and beverage \\
\hline DXA & Dual-energy X-ray absorptiometry \\
\hline EBF & Exclusively breast-fed \\
\hline EFF & Exclusively formula fed \\
\hline FEG & Federal expert group \\
\hline FF & Formula fed \\
\hline FFM & Fat-free mass \\
\hline FM & Fat mass \\
\hline FMZ & Fat mass z-score \\
\hline HAZ & Height-for-age z-score \\
\hline HC & Head circumference \\
\hline HHS & Department of Health and Human Services \\
\hline LAZ & Length-for-age z-score \\
\hline NEL & Nutrition Evidence Library \\
\hline NESR & Nutrition Evidence Systematic Review \\
\hline NIH & National Institutes of Health \\
\hline P/B-24 & Pregnancy and Birth to 24 Months Project \\
\hline RCT & Randomized controlled trial \\
\hline TEC & Technical Expert Collaborative \\
\hline USDA & United States Department of Agriculture \\
\hline WAZ & Weight-for-age $z$ score \\
\hline WC & Waist circumference \\
\hline WHZ & Weight-for-height $z$ score \\
\hline & Weight-for-length z score \\
\hline
\end{tabular}




\section{WHAT IS THE RELATIONSHIP BETWEEN THE TIMING OF INTRODUCTION OF COMPLEMENTARY FOODS AND BEVERAGES AND GROWTH, SIZE, AND BODY COMPOSITION?}

\section{PLAIN LANGUAGE SUMMARY}

What is the question?

- The question is: What is the relationship between the timing of introduction of complementary foods and beverages (CFB) and growth, size, and body composition?

What is the answer to the question?

- Moderate evidence suggests that first introduction of any CFB between 4-5mo compared to approximately $6 \mathrm{mo}$ of age is not associated with weight status, body composition, body circumferences, weight, or length among generally healthy, full-term infants.

- Limited evidence suggests that introducing CFB before $4 \mathrm{mo}$ of age may be associated with higher odds of overweight/obesity.

- There is not enough evidence to determine the relationship between introduction of CFB at $7 \mathrm{mo}$ of age or older on growth, size, or body composition.

Why was this question asked?

- This important public health question was identified and prioritized as part of the U.S. Department of Agriculture and Department of Health and Human Services Pregnancy and Birth to 24 Months Project.

How was this question answered?

- A team of Nutrition Evidence Systematic Review staff conducted a systematic review in collaboration with a group of experts called a Technical Expert Collaborative

What is the population of interest?

- Generally healthy infants and toddlers who were fed complementary foods and beverages from ages $0-24 \mathrm{mo}$ and had growth, size, and/or body composition outcomes measured across the lifespan

What evidence was found?

- Eighty-one articles examined the association between timing of introduction of CFB, as early as $1 \mathrm{mo}$ and as late as $12 \mathrm{mo}$ of age, and growth, size, and/or body composition outcomes across the lifespan

- In the majority of studies, no significant associations were reported. A limited number of observational studies suggested that CFB introduction before age $4 \mathrm{mo}$ was associated with higher odds of overweight/obesity. No conclusion was drawn regarding the introduction of CFB at 7 mo or older.

- Limitations in the evidence included inconsistency among all studies and a preponderance of null findings 
How up-to-date is this review?

- This review includes literature from 01/1980 to 07/2016 


\section{TECHNICAL ABSTRACT}

\section{Background}

- Systematic reviews were conducted as part of the U.S. Department of Agriculture and Department of Health and Human Services Pregnancy and Birth to 24 Months Project.

- The goal of this systematic review was to examine the following question: What is the relationship between timing of introduction of complementary foods and beverages (CFB) and growth, size, and body composition?

- Complementary feeding is the process that starts when human milk or infant formula is complemented by other foods and beverages, beginning during infancy and typically continuing to 24 months of age. CFB were defined as foods and/or beverages other than human milk or infant formula (liquids, semisolids, and solids) provided to an infant or young child to provide nutrients and energy.

\section{Conclusion Statement and Grades}

- Moderate evidence suggests that first introduction of any complementary food or beverage (CFB) between 4-5 months compared to approximately 6 months of age is not associated with weight status, body composition, body circumferences, weight, or length among generally healthy, full-term infants.

\section{Grade: Moderate}

- Limited evidence suggests that introducing CFB before 4 months of age may be associated with higher odds of overweight/obesity. Grade: Limited

- There is not enough evidence to determine the relationship between introduction of CFB at 7 months of age or older on growth, size, or body composition. Grade: Grade Not Assignable

\section{Methods}

- This systematic review was conducted by a team of staff from the Nutrition Evidence Library in collaboration with a Technical Expert Collaborative.

- Literature search was conducted using 4 databases (PubMed, Cochrane, Embase, and $\mathrm{CINAHL}$ ) to identify articles that evaluated the intervention or exposure of timing of CFB introduction and the outcomes of developmental milestones. A manual search was conducted to identify articles that may not have been included in the electronic databases searched. Articles were screened by two analysts? independently for inclusion based on pre-determined criteria.

- Data extraction and risk of bias assessment were conducted for each included study, and both were checked for accuracy. The body of evidence was qualitatively synthesized to inform development of a conclusion statement(s), and the strength of evidence was graded using pre-established criteria evaluating the body of evidence on risk of bias, adequacy, consistency, impact, and generalizability.

\section{Summary of Evidence}

- This review includes 81 articles that examined the association between timing of introduction of CFB and growth, size, and/or body composition across the 


\section{lifespan}

- Timing of CFB introduction is the age at which any or specific types of CFB were first consumed and was examined as early as 1 month and as late as 12 months of age.

- Timing of CFB introduction was not associated with growth, size, body composition, and/or weight status in the majority of included studies. A limited number of observational studies suggested that CFB introduction before 4 months of age was associated with higher odds of overweight/obesity.

- Given the normal variation in healthy child growth patterns, caution should be used when interpreting results between timing, types and/or amounts of CFB and outcomes for individuals based on findings at the population level. 


\section{FULL REVIEW}

\section{Systematic review question}

What is the relationship between timing of introduction of complementary foods and beverages and growth, size, and body composition?

\section{Conclusion statement}

Moderate evidence suggests that first introduction of any complementary food or beverage (CFB) between 4-5 months compared to approximately 6 months of age is not associated with weight status, body composition, body circumferences, weight, or length among generally healthy, full-term infants.

Limited evidence suggests that introducing CFB before 4 months of age may be associated with higher odds of overweight/obesity.

There is not enough evidence to determine the relationship between introduction of CFB at 7 months of age or older on growth, size, or body composition.

\section{Grades}

Moderate- Introduction of CFB between 4-5 compared to 6 months of age

Limited - Introduction of CFB before 4 months of age

Grade Not Assignable - Introduction of CFB at 7 months of age or older

\section{Summary}

- This review includes 81 articles that examined the association between timing of complementary foods and beverages (CFB) introduction and growth, size, and/or body composition from birth through adulthood, including:

- Five articles from two randomized controlled trials (RCTs)

- 71 prospective cohort studies

- One retrospective cohort study

- One nested case-control study

- Three case-control studies

- Timing of CFB introduction is the age at which any or specific types of CFB were first consumed and was examined as early as 1 month and as late as 12 months of age. Studies that examined the introduction of specific types or amounts of CFB are addressed in a separate review.

- Outcomes included weight, length/height, body circumferences (e.g., head, waist, chest, arm, thigh), adiposity (e.g., fat mass, skinfold thickness), weight-toheight ratio (e.g., body mass index (BMI), weight-for-length Z-score (WLZ)), and weight status (e.g., risk of overweight or obesity). Outcomes were assessed at a range of ages from birth to $42 y$ of age at either a single, pre-determined time point or at multiple time points or as continuous variables to capture change over time. Having data both before and after introduction of CFB is very important, as outcomes that do not take into account the infant's initial anthropometric status (before any CFB are introduced) are hard to interpret (e.g., risk of reverse causation) 
- Timing of CFB introduction was not associated with growth, size, body composition, and/or weight status in the majority of included studies.

- A limited number of observational studies suggested that CFB introduction before 4 months of age was associated with higher odds of overweight/obesity. However, due to inconsistency in findings and methodological limitations, a stronger conclusion was not made considering the preponderance of studies with null findings.

- There is insufficient evidence to support any association between timing of CFB introduction and body circumferences among generally healthy, full-term infants.

- Given the normal variation in healthy child growth patterns, caution should be used when interpreting results between timing, types and/or amounts of CFB and outcomes for individuals based on findings at the population level.

- Many studies examined the relationship between timing of CFB introduction and only one outcome, primarily weight, which, without information about other aspects of growth (i.e., length/height, or weight for length/height), provided limited information towards understanding how timing of CFB introduction may impact overall growth patterns. Therefore, studies that examined multiple outcomes, including outcomes that could be used to determine whether growth was healthy or unhealthy (e.g., risk of obesity, fat mass) were weighted more heavily in drawing conclusions from the body of evidence.

- Additional factors, which may mediate or moderate the relationship between the time at which CFB are introduced and growth, size, body composition, and/or weight status outcomes need to be considered including:

- Specific types and/or amounts of CFB introduced

- Early infant milk feeding practices (human milk, formula, and/or mixed)

- Parental feeding styles and the rationale for determining when to initiate CFB (e.g., perceived infant readiness, infant growth or size, fear of inadequate milk supply, sleep promotion, responsive feeding, infant food neophobia/acceptance)

- Early infant growth patterns

- Cultural preference related to parental history and/or feeding practices

\section{Description of the evidence}

This systematic review includes 81 articles that examined the association between the timing of introduction to CFB and growth, size, body composition, and/or weight status from infancy through adulthood. These articles include two RCTs, which are reported in five articles (1-5); 71 prospective cohort studies (6-76), one retrospective cohort study (77), one nested case-control study (78), and three case-control studies (79-81) (Supplemental Table S1). Multiple articles reported data from the same cohort but presented different types of outcomes, the same outcomes at different ages, or 
different outcomes at different ages $(21,24,25,30,49,51,56,60)(9,18,19,63)(10$, $12,28,31,32,35,57)$.

\section{Description of subject characteristics}

Sixty-eight articles were from studies conducted in countries categorized as "very high" according to the Human Development Index, which is a composite measure of life expectancy, education, and standards of living of countries (82): Australia (8, 29, 62, 68); Canada (27, 42-45, 58, 75); Denmark (11, 20, 61); Finland (50, 59); Germany (40); Iceland (2-5, 38); Ireland (48); Israel (22); Norway (46); Netherlands (9, 17-19, 63); U.K. (10, 21, 31-33, 35, 39, 49, 56, 57, 60, 66, 67, 70); Scotland (6, 26); U.S. (1, $7,12-14,16,23-25,28,30,36,37,47,51,53,55,71,73,74,77,79)$. Nine studies were conducted in countries categorized as "high" according to the Human Development Index (82): Brazil (52, 64); China (76, 80, 81); Iran (41); Mexico (15, 65); Peru (54).

Four multi-site studies were conducted with participants from various countries. Two studies included participants from European countries all categorized as "very high" $(34,78)$, one study included participants from three countries categorized as "very high" or "high" (72), and one study combined participants from seven countries categorized as "very high", "high", "medium", and "low" (69).

The majority of studies included participants who were healthy, full-term infants, though six prospective cohort studies did not report gestational age and birthweight of participants $(15,21,62,66,70,71)$. Almost all studies enrolled both girls and boys ( 43-55\% female), with one study including only girls (23).

Studies varied in terms of whether infants were fed human milk, infant formula, or both. Most studies included participants regardless of their feeding method and adjusted for feeding method in the analyses. However, several studies only included infants who were either exclusively breastfed (EBF) $(2-5,41,55,64,69)$ or exclusively formula-fed (EFF) $(1,33,73,74)$. In a few studies, the relationship between timing of CFB introduction and growth, size, and/or body composition was analyzed separately in BF and FF infants $(14,36,37)$.

\section{Description of timing of introduction of complementary foods and beverages}

Timing of CFB introduction was determined as the age at which CFB was first introduced. However, studies differed in terms of whether timing was analyzed categorically or continuously, what categories of ages were evaluated and compared, and the methods used for determining age of CFB introduction, whether prospectively or retrospectively. For specific time points of exposures, see Supplemental Table 1.

One RCT (reported in 4 articles) randomized EBF infants to either receive CFB starting at 4 mo of age or remain EBF from 4 until 6 mo of age (2-5). The second RCT randomized EFF infants to either receive CFB at age $16 \mathrm{wk}$, or remain EFF from 16 to 26 wk of age (1). Sixty-three observational studies analyzed age of CFB introduction categorically, often considering introduction of CFB before $4 \mathrm{mo}$ as the earliest category and at/after $6 \mathrm{mo}$ as the latest category. Categories of timing of CFB introduction varied across studies, which examined two (e.g., $<4$ mo vs. at/after 4 mo of age), three (e.g., <2 vs. $3-5$ vs. $>6$ mo), or four (1-8 vs. 9-16 vs. $17-24$ vs. $25-32$ wk) comparison groups. Few articles attempted to achieve greater specificity in terms of timing by further differentiating categories of CFB introduction within the 4-6 mo 
timeframe. There were a number of studies that examined more extreme categories of introducing CFB as early as $\leq 1 \mathrm{mo}(44,56)$ or $\leq 2 \mathrm{mo}(49,68,69,75)$ to as late as at/after 12 mo of age (15). Timing of CFB introduction was analyzed continuously in 16 studies $(7,14,16,24,25,42,43,45,50,61,62,65,66,72-74)$. Several studies examined timing of CFB introduction both continuously and categorically $(24,34,36$, $45,50,61)$. Two studies did not clearly define their methods for analyzing timing of CFB introduction $(27,57)$.

\section{Description of growth, size, and body composition outcomes}

Outcomes that were reported in relation to timing of CFB introduction ranged in age from birth to 42 y (see Supplemental Table S1). As defined by the included studies, outcomes relevant to 'growth' were examined over multiple time-points or intervals between baseline and endpoint of the study, outcomes relevant to 'size' were examined as an absolute measurement at one point in time, and 'body composition' outcomes were examined via proxies or indices, both across time and at single time points. Studies varied in how they accounted for growth beyond absolute size, such as by measuring outcomes at or across multiple time points, adjusting for baseline weight or earlier growth, or adjusting for birth weight. Therefore, the outcomes are discussed in the following categories, utilized herein to describe the included evidence: weight status, body composition (including adiposity, weight relative to length/height, and waist circumference (WC)); weight and length or height measures; and body circumferences (except waist).

\section{Weight status}

Twenty-eight articles in this SR (1 RCT; 27 from observational studies) reported weight status $(2,6,12,15,18,21,24,25,30,35,37,45,48,50-53,56,58,61,62,68,76$ 81). The relationships between timing of introduction of CFB and incidence of malnutrition, growth faltering, stunting, or wasting were not reported by studies included in this SR. The majority of studies published after 2007 used either BMI and/or BMI z-score (BMIZ) to classify participants older than $2 \mathrm{y}$ of age as having obesity if $\geq 95^{\text {th }}$ percentile or overweight if $\geq 85^{\text {th }}$ percentile. However, cut-points varied slightly across studies according to international standard references at the time the studies were conducted or respective country growth references (e.g., Centers for Disease Control and Prevention (CDC), International Obesity Task Force (IOTF), World Health Organization (WHO), National Center for Health Statistics (NCHS)). One study classified obesity as a weight-for-age at or above the $98^{\text {th }}$ percentile and computed models based on child BMI to compare with WHO, CDC, and IOTF cut-offs (30). In another study, risk of overweight was defined as having a $\mathrm{BMI} \geq 85^{\text {th }}$ percentile, and obesity was defined as having a combination of $\mathrm{BMI} \geq 85^{\text {th }}$ percentile and skinfolds (triceps and subscapular) $\geq 90^{\text {th }}$ percentile (52).

\section{Body composition}

Adiposity. Nineteen articles in this SR (2 from RCTs; 17 from observational studies) assessed adiposity with measures of fat mass (FM) or skinfold thickness $(1,5,7,9$, $10,14,17-20,37,42,43,49,57,67,70,75,77)$. A wide variety of assessment methods were used including dual-energy X-ray absorptiometry (DXA) to derive FM, FM index (FMI), lean mass, lean mass index, and/or body fat percentage $(18,49,57$, 77 ), total body water to obtain FM, fat free mass (FFM), and/or lean mass $(5,67)$, bioelectrical impedance analysis (BIA) to calculate body fat percentage (70), or a combination of DXA, total body water, and/or bioelectrical impedance to calculate FM, 
FFM, FMI, FFMI, FM Z-score (FMZ), or FFM Z-score (FFMZ) $(14,17,20)$. One article used DXA to examine android-gynoid fat ratio (18). Studies used calipers by trained staff to measure triceps and subscapular $(1,9,10,14,19,67)$, suprailiac $(1,9,19)$, bicep (19), and/or flank and quadriceps skinfold thicknesses (14), or sum of skinfold thicknesses $(7,9,19,37,42,43,75)$.

Weight-to-height. Twenty-nine articles in this SR (2 from RCTs; 27 from observational studies) assessed weight-to-height indices such as BMl and weight-for-length z-score (WLZ) $(2,5,7,17,18,21,23,29,33,34,36-38,42-44,46,55,57,60,61,63,64,68$, 70-72, 76, 77), with most using standard procedures to calculate BMI or WLZ from measurements. Several studies used weight and length or height from parent-report (76), clinical/school records $(46,81)$, a combination of measurements and self-report (61), or did not describe how they obtained weight and length/height (6).

Waist circumference (WC)/abdominal fat. Five articles in this SR (1 RCT; 4 from observational studies) assessed abdominal fat and/or WC via ultrasound (18), measured WC $(1,15)$, self-reported WC $(61)$, or by calculating a sum of core skinfold thickness measures to reflect 'central FM' (19).

\section{Weight and length or height}

Twenty-eight articles in this SR (4 from RCTs; 24 from observational studies) examined both weight and length or height outcomes $(1,2,4,5,8,10,13,14,16,17$, $23,33,34,36-38,41,44,47,55,59,64,65,67,69-72)$. The majority of studies reported growth and/or size outcomes at or in the first year of life, though five studies reported growth and/or size during the second year of life and four studies reported growth and/or size during childhood, between 2-7 y of age. Most studies reported using standard procedures to obtain weight and height, (e.g., duplicate measures from calibrated scales/stadiometers). However, a few studies obtained weight and/or length via parent-report $(11,28,39)$, clinical/school records $(47,71)$, unstandardized methods (44), or did not describe their methods $(1,33)$. Nine studies calculated weight-for-age $z$ scores (WAZ) and eight studies calculated height/length-for-age Z-scores (HAZ/LAZ) from either measured or reported weight and/or length.

\section{Head, arm, and thigh circumference}

Eight studies reported head circumference growth and/or size $(1-5,13,14,44)$. Two of these studies also reported chest, arm, and thigh circumferences $(1,14)$. Most studies reported using standard procedures to obtain head, arm, or thigh circumferences (e.g., non-stretchable tape and standard landmarks).

\section{Evidence synthesis}

Results from the included studies, organized by study design and similar outcomes, are described in Table 1.

\section{Randomized controlled trials}

In the RCTs, EBF infants (2-5) were randomized to start receiving CFB at 4 vs. 6 mo of age or EFF infants were randomized to start receiving CFB at 16 wk vs. 26 wk of age (1). Results from four articles (one RCT) among BF infants (2-5) showed no significant associations between timing of CFB and weight, length, or $\mathrm{HC}$ at 6 mo (5), from 0-6 mo, or from 4-6 mo (4); BMI, lean mass, or FM at 6 mo (5); WAZ/LAZ at 18 mo or change in WAZ/LAZ from 29-38 mo (2); weight status at $18 \mathrm{mo}$ or from 29-38 mo (2); $\mathrm{HC}$ z-score at $6 \mathrm{mo}(5), \mathrm{HC}$ or $\mathrm{HC}$-for-age at $18 \mathrm{mo}(2,3)$; or change in $\mathrm{HC}$ from 29-38 
mo (2).

In the trial with EFF infants, timing of CFB was examined relative to weight, length, adiposity, and body circumference outcomes (1). Significant findings were reported for isolated outcomes. The EFF infants who were introduced to CFB at 16 wk vs. 16-26 wk had increased change in length and a smaller decrease in suprailiac skinfold thickness from 16-26 wk of age. Notably, groups significantly differed in length by $1 \mathrm{~cm}$ at baseline and these differences were not clearly accounted for so the results should be interpreted with caution. Further, the groups did not differ in length at 26 wk. No other significant differences between groups were found in relation to additional outcomes including total skinfold thickness, or change in skinfold thickness, weight, or body circumferences from 16-26 wk of age. Several of these articles from both RCTs $(1,4,5)$ reported outcomes at or before 26 wk of age, when accumulation of FM is maximal relative to body weight, which makes drawing a stronger conclusion on inadequate or excessive growth, size, body composition, and/or weight status difficult without additional follow-up measurements over time.

\section{Observational studies}

\section{Weight status}

Twenty-seven articles from observational studies examined the relationship between timing of CFB introduction and weight status outcomes $(6,12,15,18,21,24,25,30$, $35,37,45,48,50-53,56,58,61,62,68,76-81)$. About half of these 27 articles reported no association between timing of CFB introduction and weight status, such that early introduction of CFB (primarily $<4$ mo of age) was not significantly associated with risk of overweight/obesity during childhood, at ages ranging between 2-14 y (12, $15,18,21,25,45,48,50,52,56,58,77,78,80)$. The remaining articles reported significant negative associations between timing of CFB and weight status, such that earlier introduction of CFB (primarily $<4$ mo of age although timing of CFB varied) was significantly associated with higher weight status $(6,30,35,37,51,53,61,62,68,76$, $79,81)$. Higher odds of overweight/obesity in those with earlier CFB introduction were observed primarily in childhood between 2-6 y of age (range of 2-42 y). Several articles included multiple comparison groups (e.g., introduction of CFB <4, 4-6, >6 mo) but found higher odds of overweight/obesity only for those who were in the earliest CFB introduction group $(6,37,51,68,76,81)$. A few of these articles reported additional null associations, depending on the comparison group and/or age of outcome.

Across the studies that examined timing of CFB introduction relative to weight status outcomes, there was heterogeneity in categories of CFB introduction, age of outcome assessment (ranging from 2-42 y), methods used for outcome assessment (e.g., weight status derived from either measured vs. reported weight and height), and variable cut-offs for either BMI or BMIZ to classify obesity and/or overweight separately or as one "above healthy" category of weight status. Two studies examined age of introduction of sugar-sweetened beverages (SSBs) specifically rather than CFB generally $(15,53)$.Very few of these studies examined other outcomes that may help with interpretation of weight status. For instance, some articles that examined timing of introduction of CFB in relation to weight status also examined timing of CFB in relation to adiposity $(18,37,77)$ and/or weight $(37,48,50)$. Most of those studies found no significant association between timing of CFB and adiposity during childhood (3-6 y) or weight during the first 3 y of life. One study showed no significant association between 
timing of CFB introduction (either $<4,<5$, or $<6 \mathrm{vs}$. $\geq 6 \mathrm{mo}$ ) and weight status at 3 y but observed increased weight at 9 mo (48). Huh et al. reported that earlier CFB introduction ( $<4$ vs. $4-5$ mo but not vs. $\geq 6 \mathrm{mo}$ ) in FF infants was associated with increased weight status, BMI, BMIZ, and WAZ at 3 y but results in BF infants were null (37). In either BF or FF infants in that study, timing of CFB was not significantly associated with skinfold thickness at $3 \mathrm{y}$. Studies also varied in adjusting the results for potentially key confounding factors. For example, all of the articles that reported significant results adjusted for birth weight, but did not consistently adjust for baseline weight, length or other measures of weight status. In addition, studies varied in the magnitude of observed effects.

\section{Body composition}

Adiposity. Seventeen observational studies examined the relationship between timing of CFB introduction and adiposity outcomes $(7,9,10,14,17-20,37,42,43,49,57,67$, $70,75,77)$, of which the majority reported no significant association between timing of CFB introduction and adiposity outcomes in childhood (age ranges between 6 mo and $15 \mathrm{y}$ ), including skinfold thickness, FM and FFM, lean mass and FM indices, and percentage of body fat $(7,9,10,14,18,20,37,43,49,57,67,75,77)$. Three of the 17 articles reported significant negative associations between timing of CFB and either skinfold thickness at $12 \mathrm{mo}$ (52) or $24 \mathrm{mo}$ (19), or body fat percentage at 7 y (70), One of the 17 articles also reported a negative association such that later CFB introduction $>6$ mo vs. < 4 mo was associated with lower FMZ at 5-6y, but this was no longer significant in the final model (17). These studies (4 of 17) found no significant associations when examining other related outcomes (e.g., peripheral FM at $24 \mathrm{mo}$ (19), BMl at 12 mo (42), FFMl at 5-6 y (17)).

Across the studies that assessed timing of CFB relative to adiposity, a number of studies had substantial methodological limitations such as limited generalizability from using a very early (<4 mo) age of introduction of CFB $(10,17,49,57,67,70,75,77)$. Several examined additional outcomes including weight-to-height ratios, weight, length, and/or central adiposity $(7,10,14,17,19,37,43,57,67,70,77)$. Of those studies that examined additional outcomes, the results for adiposity outcomes were primarily null and inconsistent in terms of direction and/or sub-group of interest (e.g., FF vs. BF) relative to other outcomes. For example, Agras et al. (7) found that age of (continuous) introduction of CFB was not significantly associated with skinfold thickness at any age but delayed introduction was positively associated with $\mathrm{BMI}$ at 1 , 2 , and $3 \mathrm{y}$ of age. However, it is unclear what drove those findings with respect to $\mathrm{BMI}$ because Agras et al. (7) did not report weight or height outcomes. Baird et al. (10) found null results between timing of CFB ( $<3$, at 3 , at $4 \mathrm{vs}$. $\geq 5 \mathrm{mo}$ ) and both adiposity and weight from birth -6 mo of age, but found that timing of CFB (at 3 vs. $\geq 5 \mathrm{mo}$ ) was associated with gain in length from birth- 6 mo of age.

There was substantial variation in the adiposity outcomes reported and corresponding assessment methods. For example, some studies reported FM measured via DXA or bioelectrical impedance while others reported FM via sum of skinfold thickness. There were no clear patterns observed based on the technique used to measure of adiposity.

Weight-to-height. Twenty-seven articles from observational studies examined the relationship between timing of CFB introduction and weight-to-height outcomes $(7,17$, $18,21,23,29,33,34,36-38,42-44,46,55,57,60,61,63,64,68,70-72,76,77$ ) of which the majority reported no significant associations between timing of CFB 
introduction and BMI or BMIZ mostly in early childhood (ages ranged between 2 and $11.5 \mathrm{y})$.

Several of the 27 observational studies reported inconsistent but significant associations between timing of CFB and/or BMI, BMIZ, or WLZ. Most of the significant findings identified were negative associations, with earlier CFB introduction significantly associated with higher BMI at 3 y (37), $4-5$ y (76), and 42 y (61), BMIZ at 36 mo (34), and WLZ from 9-12 mo (44). However, across the studies with significant findings, a few positive, null, and/or mixed associations were also reported $(7,33,34$, 55). See Table 2 for more details. Grote et al. (33) reported a significant negative association between timing of CFB introduction ( $\leq 13 \mathrm{vs}$. $\geq 22 \mathrm{wk}$ ) and BMl-for-age trajectory until 24 mo but found no relationship relative to WLZ, BMI or BMIZ at 24 mo of age. Notably, most of the studies with significant associations (in either direction) compared groups of CFB introduction at either very early ages such as $<4$ mo relative to various other ages, or at unique ages such as 9 mo. In addition, most of the studies with any significant finding relative to weight-to-height indices had substantial methodological limitations such as limited generalizability and potential for reverse causality.

Across the studies that examined timing of introduction of CFB and weight-to-height, there was heterogeneity in terms of: age of outcome assessment (6 mo - $42 \mathrm{y})$, methods used for outcome assessment (e.g., BMl obtained from measured vs. selfreported weight and height), type of CFB introduced (e.g., juice, cereal, meat), and which potential key confounders were adjusted for in analyses. For instance, the majority adjusted for birth weight in addition to baseline anthropometric values but did not account for maternal age, gestational age, or parental weight/height status in analyses. Most of the studies that examined weight-to-height indices examined additional outcomes, such as adiposity, weight, and/or length or height. Many, but not all, of those studies examined weight and/or length or height outcomes at the same or different ages. Results were inconsistent across these studies for weight-to-height indices and weight. For example, although Grote et al. (33) found no significant association of timing of introduction of CFB with respect to BMI, WLZ, or BMIZ at 24 mo, those who were introduced to CFB earlier vs. later ( $\leq 13 \mathrm{vs.} 14-17 \mathrm{wk}$ ) or later vs. earlier (18-21 vs. 14-17 wk) had higher WAZ at 24 mo of age. Haschke et al. (34) reported that EBF infants introduced to CFB $<4$ mo compared to those who remained EBF from 4-6 mo had lower BMIZ at each mo assessed (between 1 and 36 mo of age), and lower WAZ at $3,4,5$, and 6 mo of age, but no significant association with WAZ at younger or older ages (birth, 1, 2, 9, 12, 18, 24, 30, or $36 \mathrm{mo}$ ). In addition, Haschke also reported significant positive correlations between age of CFB introduction and BMI gain $\left(\mathrm{kg} / \mathrm{m}^{2} / \mathrm{y}\right)$ and weight gain $(\mathrm{g} / \mathrm{mo})$ from 1-12 mo, but these associations were not significant for outcomes from 1-24 mo or 1-36 mo of age. A few studies examined only weight-to-height (length) indices, all of which had null findings $(29,46,60,63)$.

Because weight-to-height indices are a proxy for body composition, results from these studies are difficult to interpret given the different implications of body composition early in infancy/childhood as opposed to adulthood. Weight-to-height indices, such as $\mathrm{BMI}$, alone provide limited information without other relevant outcomes (e.g., weight status, adiposity, weight and length outcomes).

Waist circumference (WC)/Abdominal fat. Four observational articles examined the 
relationship between timing of CFB introduction and central adiposity outcomes (15, $18,19,61)$, of which three reported no significant associations with WC at 24 mo or $42 \mathrm{y}$, or abdominal fat at $6 \mathrm{y}$ or $8-14$ y $(15,18,19,61)$. Earlier CFB introduction, measured continuously, was associated with increased WC at $42 \mathrm{y}$ in one study (61). However, that study had substantial methodological limitations including use of nonstandardized anthropometric assessments (e.g., self-report) and limited generalizability due to CFB practices at the time of original data collection (e.g., 19591960). Two of the studies had very long time to follow-up at 8-14 y and $42 \mathrm{y}$ and reported associations for categories of age of CFB introduction at very early $(<4 \mathrm{mo})$ or very late $(<12 \mathrm{mo})$ ages $(15,61)$. Across these four observational studies, there was variability in terms of methods used to measure central adiposity, age of outcome assessment and methodological limitations.

\section{Weight and length/height}

Twenty-four articles from observational studies examined the relationship between timing of CFB introduction and both weight and length or height outcomes $(8,10,13$, $14,16,17,23,33,34,36-38,41,44,47,55,59,64,65,67,69-72)$, of which the majority (16 studies) reported at least one significant association with weight and/or length $(8,10,13,16,17,33,34,36-38,44,55,59,65,69,70)$ and eight reported null findings for both weight and length $(14,23,41,47,64,67,71,72)$. Of the articles that found significant relationships, the direction of findings was inconsistent. In eight of the 24 studies, there were no significant associations between timing of CFB relative to length/height $(13,16,36,37,39,55,65,70)$, but either positive $(13,55)$ or negative associations relative to weight $(36,37,39,65,70)$. Several observational studies found negative associations between timing of CFB and length/height, such that either earlier introduction of CFB was associated with greater length/height, or later introduction of CFB was associated with shorter length/height $(18,20,27,69(8,17)$, but found either no significant association with weight $(10,59)$ or negative associations with weight $(8,17)$. The studies with mixed findings also varied based on the timing of CFB introduction, age at which outcome was assessed, or the type of CFB introduced.

Across the studies that examined the relationship between timing of introduction of CFB and weight and length/height, most studies also examined the relationship between timing of CFB introduction and at least one other relevant outcome with potential health implications (e.g., obesity, adiposity, weight-to-height indices). Huh et al. examined obesity at age $3 \mathrm{y}$ in addition to weight, length, weight-to-height ratios, and adiposity outcomes (37). As noted in the section on weight status results, all of the significant associations that Huh et al. identified were found in FF infants, and no associations were found in BF infants (when comparing CFB introduction $<4$ vs. $4-5$ mo, or $\geq 6$ vs. $4-5 \mathrm{mo}$ ). Other articles also examined adiposity outcomes, in addition to weight and length $(10,14,17,67,70)$. A few studies shared the same direction of findings for weight and adiposity but the findings across these studies were inconsistent in terms of direction, age of outcome assessment (e.g., weight at 6-12 mo but adiposity at 5-6 y), and specific timing interval for CFB introduction. For example, Wilson et al. (70 (Wilson, 1998 \#105) found a negative association between timing of CFB ( $<15$ vs. $>15$ wk) and weight as well as FM at $7 \mathrm{y}$, but found no relationship with height at $7 \mathrm{y}$. deBeer et al. (17) found that timing of CFB ( $<4 \mathrm{vs}$. $>6 \mathrm{mo}$ ) was negatively associated with conditional weight at 6-12 mo, height at 6-12 mo, and FMZ at 5-6 y, but the association with FMZ at 5-6y was no longer significant after fully adjusting models and there were no associations with other outcomes (e.g., BMIZ, FFMZ). 


\section{Head, arm, and thigh circumference}

Timing of CFB introduction was not significantly associated with head circumference $(\mathrm{HC})$ in most of the articles in this SR that examined body circumferences (8 articles total: 3 from observational studies discussed here and 5 from 2 RCTs previously discussed). These null findings were observed at various ages, ranging from approximately 6 mo to $\sim 3$ y of age, and when $\mathrm{HC}$ was examined at a single time point, or when change in $\mathrm{HC}$ over time was considered. One observational study (44) reported that introduction of cereal at $3 \mathrm{mo}$ of age, but not at 6 or $9 \mathrm{mo}$, was associated with lower HC from 3-6 mo. Notably, cereals consumed in that study were not representative of infant cereals consumed in the U.S. and only $1.2 \%$ of the children were consuming cereals at $3 \mathrm{mo}$. In addition, that study is limited by the potential for reverse causality and use of unstandardized anthropometric assessments.

Only two studies in this SR, one from an RCT previously discussed and one observational study, examined other circumferences (arm, thigh, and chest); differences between groups were not significant at $26 \mathrm{wk}$, from 16-26 wk, or between $6-24$ mo of age $(1,14)$. Although these two studies had minimal methodological limitations, they varied in study design and assessment of timing of CFB. 
Table 1. Studies that examined the timing of introduction of complementary oods and beverages and growth, size, and body composition

\begin{tabular}{|c|c|c|c|c|c|c|}
\hline $\begin{array}{l}\text { Reference, country, } \\
\text { analytic sample size }\end{array}$ & $\begin{array}{l}\text { Intervention/ } \\
\text { Exposure }\end{array}$ & Weight Status Results & $\begin{array}{l}\text { Body Composition } \\
\text { Results }\end{array}$ & $\begin{array}{l}\text { Weight } \\
\text { Results }\end{array}$ & $\begin{array}{l}\text { Length or } \\
\text { Height Results }\end{array}$ & $\begin{array}{l}\text { Head, Arm, Thigh } \\
\text { Circumference } \\
\text { Results }\end{array}$ \\
\hline \multicolumn{7}{|l|}{$\begin{array}{l}\text { Randomized } \\
\text { Controlled Trials }\end{array}$} \\
\hline $\begin{array}{l}\text { Bainbridge, } 1996 \text { (1) } \\
\text { U.S. } \\
\text { N: } 41\end{array}$ & $\begin{array}{l}\text { CFB at } 16 w k \text { vs. } \\
\text { EFF 16-26wk }\end{array}$ & & $\begin{array}{l}\text { Suprailiac skinfold } \\
\text { thickness at age } \\
26 w k, N S G D ; \text { change } \\
\text { from age } 16-26 w k,- \\
0.20 \mathrm{~mm}, \mathrm{SD}: 0.80 \text { vs. } \\
-0.92 \mathrm{~mm}, \mathrm{SD}: 1.25 \text {, } \\
\mathrm{P}=0.04 \text {. Mid-thigh, } \\
\text { triceps, or subscapular } \\
\text { skinfold thickness at } \\
\text { age } 26 w \mathrm{wk} \text { or change } \\
\text { from age 16-26wk, } \\
\text { NSGD; abdominal } \\
\text { circumference at age } \\
26 w k \text { or change from } \\
\text { age } 16-26 w k, \text { NSGD; } \\
\text { Chest, MUAC, mid- } \\
\text { thigh circumference at } \\
\text { age 26wk or change } \\
\text { from age 16-26wk, } \\
\text { NSGD }\end{array}$ & $\begin{array}{l}\text { Weight change } \\
\text { at age 26wk or } \\
\text { from age 16- } \\
26 w k, N S G D\end{array}$ & $\begin{array}{l}\text { Length change } \\
\text { at age 26wk, } \\
\text { NSGD; change } \\
\text { from age } 16- \\
26 w k, 5.03 \text {, SD: } \\
1.05 \text { vs. 3.62, } \\
\text { SD:2.72, } \\
P=0.05 \text {. }\end{array}$ & $\begin{array}{l}\mathrm{HC} \text { at age } 26 \mathrm{wk} \text { or } \\
\text { change from age } \\
16-26 w k, N S G D\end{array}$ \\
\hline $\begin{array}{l}\text { Jonsdottir, } 2012 \text { (4) } \\
\text { Iceland } \\
\mathrm{N}: 100\end{array}$ & $\begin{array}{l}\text { CFB at } 4 \mathrm{mo} \text { vs. } \\
\text { EBF } 4-6 \mathrm{mo}\end{array}$ & & & $\begin{array}{l}\text { Weight gain } \\
\text { from age birth- } \\
6 \mathrm{mo} \text { or } 4-6 \mathrm{mo} \text {, } \\
\text { NSGD }\end{array}$ & $\begin{array}{l}\text { Length gain from } \\
\text { age birth-6mo or } \\
\text { 4-6mo, NSGD }\end{array}$ & $\begin{array}{l}\text { HC gain from age } \\
\text { birth-6mo or 4- } \\
6 m o, \text { NSGD }\end{array}$ \\
\hline $\begin{array}{l}\text { Jonsdottir, } 2013 \text { (3) } \\
\text { Iceland } \\
\text { N: } 54-78\end{array}$ & $\begin{array}{l}\text { CFB at } 4 \mathrm{mo} \text { vs. } \\
\text { EBF } 4-6 \mathrm{mo}\end{array}$ & & & & & $\begin{array}{l}\text { HC gain from age } \\
\text { birth-18mo, NSGD }\end{array}$ \\
\hline $\begin{array}{l}\text { Jonsdottir, } 2014 \text { (2) } \\
\text { Iceland }\end{array}$ & $\begin{array}{l}\text { CFB at } 4 \mathrm{mo} \text { vs. } \\
\text { EBF } 4-6 \mathrm{mo}\end{array}$ & $\begin{array}{l}\text { Overweight, overweight, } \\
\text { or obesity at age } 18 \mathrm{mo} \text {, }\end{array}$ & $\begin{array}{l}\text { BMl-for-age at age } \\
18 \mathrm{mo} \text {, or } 29-38 \mathrm{mo},\end{array}$ & $\begin{array}{l}\text { WAZ at age } \\
18 \mathrm{mo}, \text { or } 29-\end{array}$ & $\begin{array}{l}\text { LAZ at age } \\
18 \mathrm{mo}, \text { or } 29-\end{array}$ & $\begin{array}{l}\text { HC-for-age at age } \\
18 \mathrm{mo}, \text { or } 29-38 \mathrm{mo},\end{array}$ \\
\hline
\end{tabular}




\begin{tabular}{|c|c|c|c|c|c|c|}
\hline $\mathrm{N}: 100$ & & or 29-38mo, NSGD & NSGD & $38 \mathrm{mo}$ NSGD & $38 \mathrm{mo}$ NSGD & NSGD \\
\hline $\begin{array}{l}\text { Wells, } 2012 \\
\text { (5) Iceland } \\
\text { N. } 100\end{array}$ & $\begin{array}{l}\text { CFB at } 4 m o \text { vs. } \\
\text { EBF } 4-6 m o\end{array}$ & & $\begin{array}{l}\text { BMI, lean mass, or FM } \\
\text { at age } 6 \text { mo, NSGD }\end{array}$ & $\begin{array}{l}\text { Weight at age } \\
6 \mathrm{mo} \text {, NSGD }\end{array}$ & Length at age & $\begin{array}{l}\text { HCZ at age } 6 \mathrm{mo} \text {, } \\
\text { NSGD }\end{array}$ \\
\hline
\end{tabular}

\section{Prospective cohort}

\section{studies}

\begin{tabular}{lll}
\hline Abraham, $2012(6)$ & $4-5$ vs. 0-3mo & Overweight/obesity at \\
Scotland & & age $4 y, O R=0.74,95 \%$
\end{tabular}

N: 3994

$\mathrm{Cl}: 0.57,0.97, \mathrm{P}=0.009$

6-10 vs. 0-3mo Overweight/obesity at

age $4 \mathrm{y}, \mathrm{OR}=0.72,95 \%$

Cl: $0.48,1.09, P=0.009$

\begin{tabular}{|c|c|c|c|c|}
\hline $\begin{array}{l}\text { Agras, } 1990(7) \\
\text { U.S. } \\
\text { N: } 54\end{array}$ & Continuous, wk & $\begin{array}{l}\text { BMI at age 1y: partial } \\
\mathrm{r}^{2}=0.096, P=0.03 ; \mathrm{BMI} \\
\text { at age } 2 \mathrm{y}: \text { partial } \\
\mathrm{r}^{2}=0.129, \mathrm{P}=0.02 ; \mathrm{BMI} \\
\text { at age } 3 \mathrm{y}: \text { partial } \\
\mathrm{r}^{2}=0.148, \mathrm{P}=0.01 ; \mathrm{BMI} \\
\text { at age } 6 \mathrm{y}: \mathrm{NSA} \\
\text { Skinfold thickness at } \\
\text { age } 1,2 \text {, or } 6 \mathrm{y}, \mathrm{NSA}\end{array}$ & & \\
\hline $\begin{array}{l}\text { Atkins, } 2016 \\
\text { (8) Australia } \\
\text { N: } 423\end{array}$ & $<6$ vs. $\geq 6 \mathrm{mo}$ & & $\begin{array}{l}\text { Weight at age } \\
\text { 20mo: mean } \\
\text { difference } \\
0.4 \mathrm{~kg}, 95 \% \mathrm{Cl} \text { : } \\
0.1,0.7 \\
\mathrm{P}=0.006\end{array}$ & $\begin{array}{l}\text { Length at age } \\
\text { 20mo: mean } \\
\text { difference of } \\
0.9 \mathrm{~cm}, 95 \% \mathrm{Cl} \text { : } \\
0.2,1.7, \mathrm{P}= \\
0.010\end{array}$ \\
\hline $\begin{array}{l}\text { Ay, } 2008(9) \\
\text { Netherlands } \\
\text { N: } 1012\end{array}$ & $<5 \mathrm{mo}$ or not & $\begin{array}{l}\text { Sum skinfold } \\
\text { thickness at age } \\
24 \mathrm{mo} \text { : NSA }\end{array}$ & & \\
\hline $\begin{array}{l}\text { Baird, } 2008 \\
(10) \text { U.K. } \\
\mathrm{N}: 1,740 \text { at } 6 \mathrm{mo} ; \\
1335 \text { at } 12 \mathrm{mo}\end{array}$ & $<3$ vs. $\geq 5 \mathrm{mo}$ & $\begin{array}{l}\text { Gain in skinfold } \\
\text { thickness from age } \\
\text { birth-6mo, or skinfold } \\
\text { thickness at age } 6 \mathrm{mo} \text { : }\end{array}$ & $\begin{array}{l}\text { Weight gain } \\
\text { from age birth- } \\
6 \mathrm{mo} \text { or mean } \\
\text { weight at } 6 \mathrm{mo} \text { : }\end{array}$ & $\begin{array}{l}\text { Length gain from } \\
\text { age birth- } 6 \text { mo or } \\
\text { mean length at } \\
\text { age } 6 \mathrm{mo}, \mathrm{NSA}\end{array}$ \\
\hline
\end{tabular}


at 3 vs. $\geq 5 \mathrm{mo}$

\begin{tabular}{|c|c|c|c|c|c|}
\hline & at 3 vs. $\geq 5 \mathrm{mo}$ & $\begin{array}{l}\text { Gain in skinfold } \\
\text { thickness from age } \\
\text { birth-6mo, or skinfold } \\
\text { thickness at age } 6 \mathrm{mo} \text { : } \\
\text { NSA }\end{array}$ & $\begin{array}{l}\text { Weight gain } \\
\text { from age birth- } \\
6 \text { mo or mean } \\
\text { weight at } 6 \mathrm{mo} \text { : } \\
\text { NSA }\end{array}$ & $\begin{array}{l}\text { Length gain from } \\
\text { age birth-6mo: } \\
\beta=0.21,95 \% \mathrm{Cl} \text { : } \\
0.02,0.39 \text {, } \\
\mathrm{P}<0.05 . \text { Mean } \\
\text { length at age } \\
6 \mathrm{mo}: \beta=0.68 \text {, } \\
95 \% \mathrm{Cl}: 0.27 \\
1.08, \mathrm{P}<0.05\end{array}$ & \\
\hline & at 4 vs. $\geq 5 \mathrm{mo}$ & $\begin{array}{l}\text { Gain in skinfold } \\
\text { thickness from age } \\
\text { birth-6mo, or skinfold } \\
\text { thickness at age } 6 \mathrm{mo} \text { : } \\
\text { NSA }\end{array}$ & $\begin{array}{l}\text { Weight gain } \\
\text { from age birth- } \\
6 \mathrm{mo} \text { or mean } \\
\text { weight at } 6 \mathrm{mo} \text { : } \\
\text { NSA }\end{array}$ & $\begin{array}{l}\text { Length gain from } \\
\text { age birth-6mo or } \\
\text { mean length at } \\
\text { age } 6 \mathrm{mo}, \mathrm{NSA}\end{array}$ & \\
\hline \multirow[t]{2}{*}{$\begin{array}{l}\text { Baker, } 2004 \\
\text { (11) Denmark } \\
\mathrm{N}: 3,768\end{array}$} & $\begin{array}{l}<16 \text { vs. } \geq 16 w k, B F \\
\text { for }<20 w k\end{array}$ & & $\begin{array}{l}\text { Weight gain } \\
\text { from age birth- } \\
\text { 1y: } \beta=365.1 \\
\text { SE: } 82.9 \\
P<0.0001\end{array}$ & & \\
\hline & $\begin{array}{l}<16 \text { vs. } \geq 16 w k, B F \\
\text { for }>20-40 w k\end{array}$ & & $\begin{array}{l}\text { Weight gain } \\
\text { from age birth- } \\
1 \mathrm{y}, \mathrm{NSA}\end{array}$ & & \\
\hline $\begin{array}{l}\text { Barrera, } 2016(12) \\
\text { U.S. } \\
\text { N: } 1181\end{array}$ & $\begin{array}{l}<4,4-<6 \text {, and } \\
\geq 6 \mathrm{mo}\end{array}$ & & & & \\
\hline $\begin{array}{l}\text { Barton, } 2002(13) \\
\text { U.S. } \\
\text { N: } 52\end{array}$ & $<4$ vs. $>4 \mathrm{mo}$ & & $\begin{array}{l}\text { Weight at age } \\
4-6 \mathrm{mo}: 263.7 \pm \\
30.9 \text { oz. vs. } \\
291.4 \pm \\
44.80 z ., P=0.04\end{array}$ & $\begin{array}{l}\text { Length at age 4- } \\
6 \mathrm{mo}: \text { NSA }\end{array}$ & $\begin{array}{l}\mathrm{HC} \text { at age 4-6mo: } \\
\mathrm{NSA}\end{array}$ \\
\hline $\begin{array}{l}\text { Butte, } 2000(14) \\
\text { U.S. } \\
\text { N: } 72\end{array}$ & $\begin{array}{l}\text { Continuous, } \\
\text { among BF or FF } \\
\text { infants }\end{array}$ & $\begin{array}{l}\text { Between age 6-24mo: } \\
\text { triceps, flank, } \\
\text { subscapular, or } \\
\text { quadriceps skinfold } \\
\text { thickness, NSA; } \\
\text { FM. FFM. \% FM. }\end{array}$ & $\begin{array}{l}\text { Between age 6- } \\
\text { 24mo: Weight, } \\
\text { NSA }\end{array}$ & $\begin{array}{l}\text { Between age 6- } \\
24 \mathrm{mo} \text { : Length, } \\
\text { NSA }\end{array}$ & $\begin{array}{l}\text { Between age 6- } \\
\text { 24mo: HC, NSA }\end{array}$ \\
\hline
\end{tabular}


NSA; Chest, arm,

thigh circumferences,

NSA

\begin{tabular}{|c|c|c|c|c|c|}
\hline $\begin{array}{l}\text { Cantoral, } 2016 \text { (15) } \\
\text { Mexico } \\
\text { N: } 227\end{array}$ & $\leq 12$ vs $>12 \mathrm{mo}$ & Obesity at 8-14y: NSA & $\begin{array}{l}\text { Abdominal obesity at } \\
\text { age } 8-14 y, \text { NSA }\end{array}$ & & \\
\hline $\begin{array}{l}\text { Carruth, } 2000 \\
\text { (16) U.S. } \\
\text { N: } 94\end{array}$ & Continuous & & & $\begin{array}{l}\text { Rate of weight } \\
\text { change from } \\
\text { age } 12-24 \mathrm{mo} \text {, } \\
\text { NSA }\end{array}$ & $\begin{array}{l}\text { length slope } \\
\text { from age 2-8mo, } \\
\text { NSA }\end{array}$ \\
\hline \multirow[t]{2}{*}{$\begin{array}{l}\text { de Beer, } 2015 \\
\text { (17) Netherlands } \\
\text { N: } 2227\end{array}$} & $>6$ vs. $<4 \mathrm{mo}$ & & $\begin{array}{l}\text { At age 5-6y: FMZ } \\
\beta=-0.23 ; 95 \% \mathrm{Cl} \text { : } \\
-0.39,-0.08, P=0.004 \\
\text { final model, NSA; } \\
\text { FFMZ, NSA; BMIZ, } \\
\text { NSA }\end{array}$ & $\begin{array}{l}\text { Conditional } \\
\text { weight } z \text {-scores } \\
\text { from age } 6- \\
12 \mathrm{mo}: \beta=-0.21 \text {, } \\
95 \% \mathrm{Cl}:-0.38 \\
-0.03, P=\mathrm{NR}\end{array}$ & $\begin{array}{l}\text { Conditional } \\
\text { height } z \text {-scores } \\
\text { from age 6- } \\
12 \mathrm{mo}: \beta=-0.20 \text {; } \\
95 \% \mathrm{Cl} \text { - }-0.37 \text {, } \\
-0.02, \mathrm{P}=\mathrm{NR} ; \\
\text { Height } z \text {-scores } \\
\text { at age } 5-6 \mathrm{y}: \beta= \\
-0.13 ; 95 \% \mathrm{Cl} \text { : } \\
-0.25,-0.01 \text {, } \\
\mathrm{P}=\mathrm{NR}\end{array}$ \\
\hline & $4-6$ vs. $<4 \mathrm{mo}$ & & $\begin{array}{l}\text { At age 5-6y, FMZ, } \\
\text { NSA; FFMZ, NSA; } \\
\text { BMIZ, NSA }\end{array}$ & $\begin{array}{l}\text { Conditional } \\
\text { weight z-scores } \\
\text { from age 6- } \\
\text { 12mo: NSA }\end{array}$ & $\begin{array}{l}\text { Conditional } \\
\text { height z-scores } \\
\text { from age 6- } \\
\text { 12mo: NSA; } \\
\text { Height z- } \\
\text { scores at 5- } \\
\text { 6y: } \beta=-0.13 \text {; } \\
\text { 95\%Cl: } \\
\text {-0.25, }-0.01 \text {, } \\
\text { P=NR }\end{array}$ \\
\hline \multirow[t]{3}{*}{$\begin{array}{l}\text { Durmus, } 2012 \text { (19) } \\
\text { Netherlands N: } 779\end{array}$} & $<4$ vs. $>5 \mathrm{mo}$ & & $\begin{array}{l}\text { Peripheral, central, or } \\
\text { total FM at ages } 6 \mathrm{mo} \\
\text { or } 24 \mathrm{mo}, \mathrm{NSA}\end{array}$ & & \\
\hline & $4-5$ vs. $>5 \mathrm{mo}$ & & $\begin{array}{l}\text { Total FM at age } 24 \mathrm{mo}: \\
\beta=1.46,95 \% \mathrm{Cl}: 0.05 \text {, } \\
2.88, P=0.08 .\end{array}$ & & \\
\hline & & & Peripheral, central, or & & \\
\hline
\end{tabular}


total FM at age $6 \mathrm{mo}$,

peripheral or central

FM at age 24mo, NSA

\begin{tabular}{|c|c|c|c|c|c|}
\hline $\begin{array}{l}\text { Durmus, } 2014 \\
\text { (18) Netherlands } \\
\text { N: } 5063\end{array}$ & $<4,4-4.9, \geq 5 \mathrm{mo}$ & $\begin{array}{l}\text { Overweight or obesity at } \\
\text { age 6y: NSA }\end{array}$ & $\begin{array}{l}\text { Total FM, android- } \\
\text { gynoid fat ratio, or BMI } \\
\text { at age 6y, NSA; } \\
\text { Abdominal fat at age } \\
6 y, \text { NSA }\end{array}$ & & \\
\hline $\begin{array}{l}\text { Ejlerskov, } 2015(20) \\
\text { Denmark } \\
\mathrm{N}: 233\end{array}$ & Continuous, mo & & $\begin{array}{l}\text { FMI or FFMI at age } \\
\text { 3y, data NR, NSA }\end{array}$ & & \\
\hline $\begin{array}{l}\text { Fairley, } 2015(21) \\
\text { U.K. } \\
\text { N: } 987\end{array}$ & $<17$ or $\geq 17 w k$ & $\begin{array}{l}\text { Overweight at age } 36 \mathrm{mo} \text { : } \\
\text { NSA }\end{array}$ & $\begin{array}{l}\text { BMIZ at age } 36 \mathrm{mo}: \\
\text { NSA }\end{array}$ & & \\
\hline \multirow[t]{2}{*}{$\begin{array}{l}\text { Fawzi, } 1997 \\
\text { (22) Israel } \\
\text { N: } 351\end{array}$} & $1 \mathrm{mo}$ vs. EBF & & & $\begin{array}{l}\text { Weight at age } \\
2 \mathrm{mo}: 4753 \text { vs. } \\
5113 \mathrm{~g}, \mathrm{P} \leq 0.05 \text {; } \\
\text { Weight at age } \\
\text { 3mo: NSA }\end{array}$ & \\
\hline & $2 \mathrm{mo}$ vs. EBF & & & $\begin{array}{l}\text { Weight at age } \\
\text { 3mo: NSA }\end{array}$ & \\
\hline $\begin{array}{l}\text { Ferris, } 1980(23) \\
\text { U.S. } \\
\text { N: } 92\end{array}$ & 2 or $>2 \mathrm{mo}$ & & $\begin{array}{l}\text { Weight/length }{ }^{2} \text { from } \\
\text { age birth-6mo: NSA }\end{array}$ & $\begin{array}{l}\text { Weight from } \\
\text { age birth-6mo: } \\
\text { NSA }\end{array}$ & $\begin{array}{l}\text { Length from age } \\
\text { birth-6mo: NSA }\end{array}$ \\
\hline \multirow[t]{2}{*}{$\begin{array}{l}\text { Flores, 2013a (24) } \\
\text { U.S. } \\
\text { N: } 6800\end{array}$} & Continuous, mo & $\begin{array}{l}\text { Overweight at } \\
\text { kindergarten entry: } \\
\text { NSA }\end{array}$ & & & \\
\hline & $\begin{array}{l}<3.5 \text { vs. } \\
\geq 3.5 \mathrm{mo} \text {, not } \\
\text { overweight at } \\
\text { preschool, } \\
\text { overweight at } \\
2 y, \text { White, no } \\
\text { maternal } \\
\text { gestational } \\
\text { diabetes }\end{array}$ & $\begin{array}{l}58 \% \text { vs. } 25.7 \% \\
\text { prevalence of } \\
\text { overweight }\end{array}$ & & & \\
\hline
\end{tabular}

Flores, 2013b Continuous, mo Obesity at kindergarten 


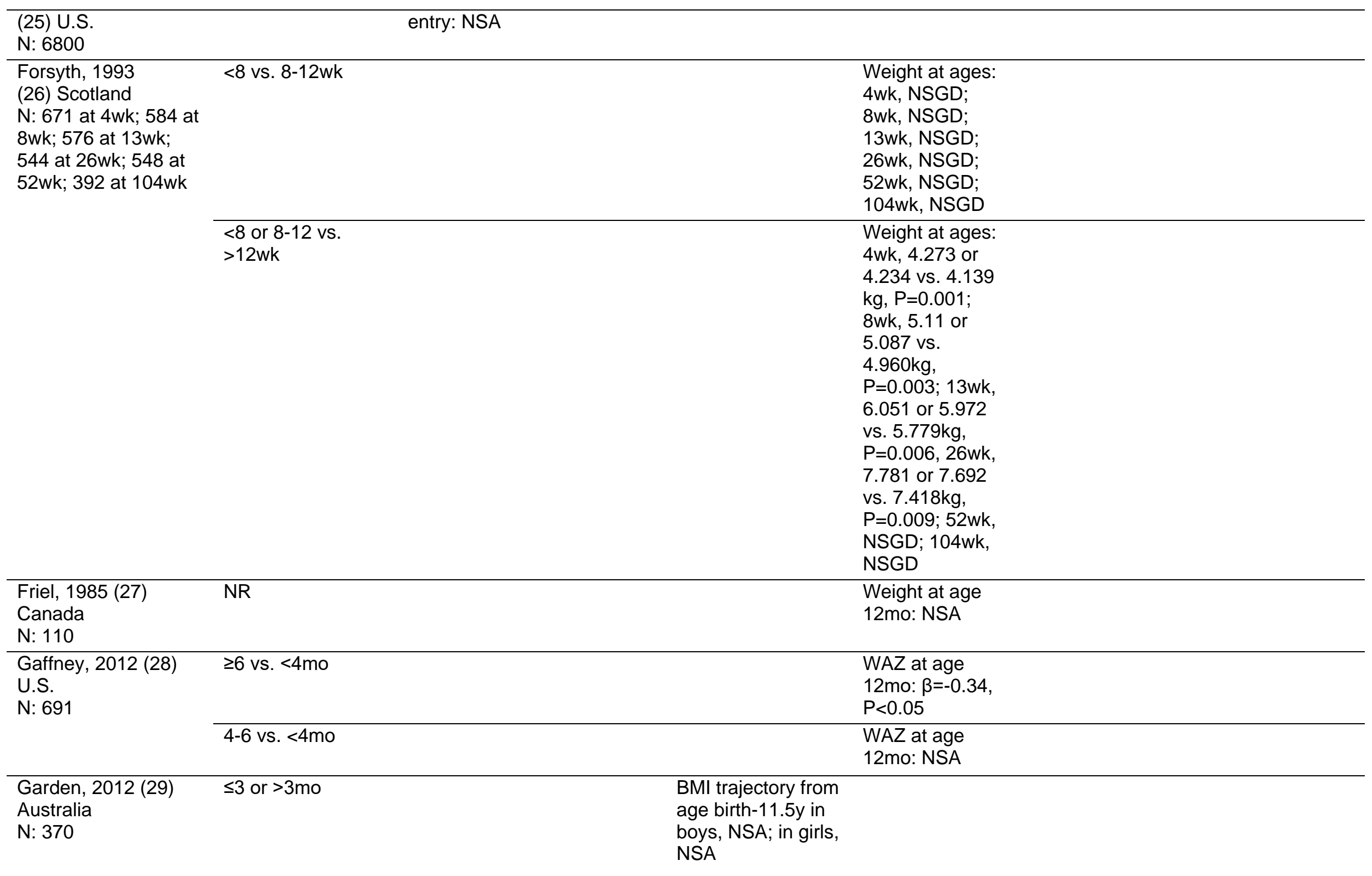




\begin{tabular}{|c|c|c|c|c|c|}
\hline $\begin{array}{l}\text { Gibbs, } 2014(30) \\
\text { U.S. } \\
\text { N: } 7,880\end{array}$ & $<4 \mathrm{mo}$ vs. not & 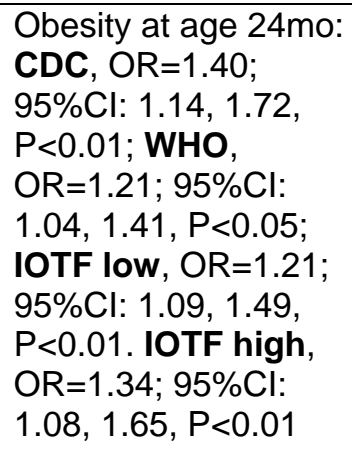 & & & \\
\hline $\begin{array}{l}\text { Griffiths, } 2009 \text { (31) } \\
\text { U.K. } \\
\text { N: } 10,533\end{array}$ & $<4$ vs. $\geq 4 \mathrm{mo}$ & & & $\begin{array}{l}\text { Conditional } \\
\text { weight gain } \\
\text { from age birth- } \\
\text { 3y: } \beta=0.07 ; \\
95 \% \mathrm{Cl}: 0.02 \text {, } \\
0.11, \mathrm{P}=0.005 \\
\text { but after } \\
\text { adjusting for } \\
\text { current child } \\
\text { height, NSA }\end{array}$ & \\
\hline $\begin{array}{l}\text { Griffiths, } 2010 \text { (32) } \\
\text { U.K. } \\
\text { N: 11,653 }\end{array}$ & $\begin{array}{l}<17.4 \mathrm{vs} . \\
\geq 17.4 \mathrm{wk}\end{array}$ & & & $\begin{array}{l}\text { Rapid weight } \\
\text { gain from age } \\
\text { 3-5y: NSA }\end{array}$ & \\
\hline $\begin{array}{l}\text { Grote, } 2011 \text { (33) } \\
\text { U.K./EU } \\
\text { N: } 687 \text {, at } 24 \mathrm{mo}\end{array}$ & $\begin{array}{l}\leq 13,14-17 \\
18-21, \text { and } \\
\geq 22 w k\end{array}$ & & $\begin{array}{l}\text { WLZ, BMI, or BMIZ at } \\
\text { age } 24 \mathrm{mo} \text { : NSA; } \\
\text { BMI-for-age } \\
\text { trajectories until age } \\
24 \mathrm{mo}, \mathrm{P}=0.011\end{array}$ & $\begin{array}{l}\text { WAZ at age } \\
24 \mathrm{mo}: \leq 13 w \mathrm{wk} \\
0.40 \pm 1.01 ; 14- \\
17 \mathrm{wk} \\
0.31 \pm 0.98 ; 18- \\
21 \mathrm{wk} \\
0.53 \pm 0.82 ; \\
\geq 22 \mathrm{wk} \\
0.23 \pm 0.91 \\
\mathrm{P}=0.027 ; \\
\text { Weight at age } \\
24 \mathrm{mo}, \mathrm{NSA} ; \\
\text { Weight velocity } \\
\text { (kg/mo) at age } \\
24 \mathrm{mo}, \mathrm{NSA} ;\end{array}$ & $\begin{array}{l}\text { LAZ at age } \\
24 \mathrm{mo:} \leq 13 w \mathrm{wk} \\
0.14 \pm 1.09 ; 14- \\
17 w \mathrm{wk} 0.18 \pm 1.01 ; \\
18-21 \mathrm{wk} \\
0.39 \pm 0.97 ; \\
\geq 22 w \mathrm{k} \\
0.18 \pm 0.93, \\
\mathrm{P}=0.049 \\
\text { Length (cm) at } \\
\text { age } 24 \mathrm{mo}, \mathrm{NSA} ; \\
\text { Length velocity } \\
\text { (cm/mo) at age } \\
24 \mathrm{mo}, \mathrm{NSA} ; \\
\text { Length-for-age }\end{array}$ \\
\hline
\end{tabular}




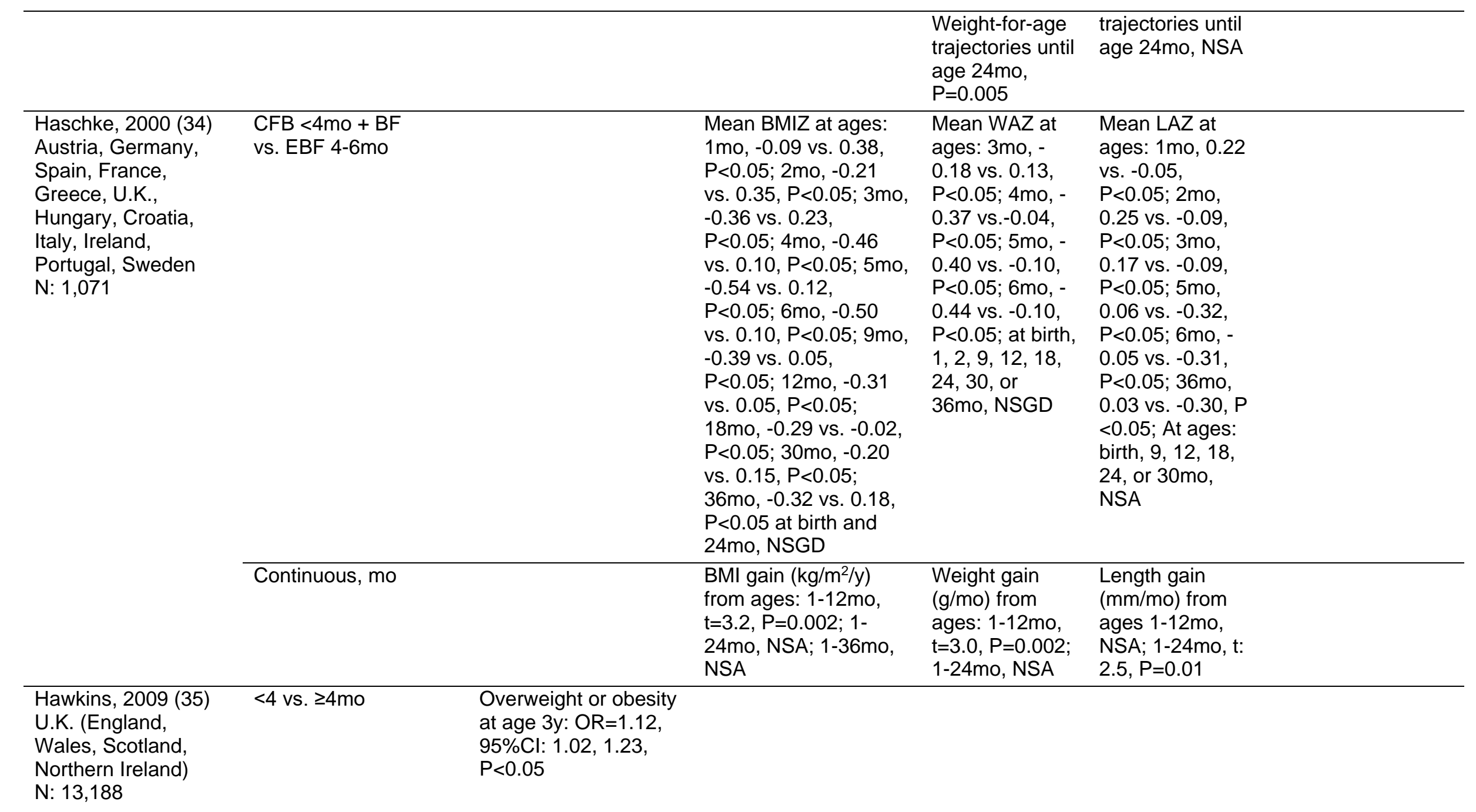




\begin{tabular}{|c|c|c|c|c|c|}
\hline \multirow[t]{2}{*}{$\begin{array}{l}\text { Heinig, } 1993(36) \\
\text { U.S. } \\
\text { N: } 87 \text { (N: } 46 \text { BF; N: } \\
41 \text { FF at 9mo) }\end{array}$} & BF: $<26$ vs. $\geq 26 w k$ & & $\begin{array}{l}\text { WLZ from age } 1- \\
18 \mathrm{mo}, \mathrm{NSA}\end{array}$ & $\begin{array}{l}\text { WAZ: from } \\
\text { age 1-18mo, } \\
\text { NSA; } \\
\text { Weight gain } \\
\text { from ages: } \\
6-9 \mathrm{mo} \text {, } \\
\text { P<0.05; } \\
\text { birth-4mo, } \\
4-6 \mathrm{mo} \text {, or } 9- \\
12 \mathrm{mo} \text {, NSA }\end{array}$ & $\begin{array}{l}\text { LAZ from age } \\
\text { 1-18mo, } \\
\text { NSA; } \\
\text { Length gain } \\
\text { from ages: } \\
\text { birth-4mo, 4- } \\
6 \mathrm{mo}, 6-9 \mathrm{mo} \text {, } \\
\text { or } 9-12 \mathrm{mo} \text {, } \\
\text { NSA }\end{array}$ \\
\hline & $\begin{array}{l}\text { FF: Continuous; } \\
<20 \text { vs. } \geq 20 w k\end{array}$ & & & $\begin{array}{l}\text { WAZ from age } \\
1-18 \mathrm{mo}, \mathrm{NSA}\end{array}$ & $\begin{array}{l}\text { LAZ from age 1- } \\
\text { 18mo, NSA } \\
\text { Length gain } \\
\text { from birth- } \\
4 \mathrm{mo}, 4-6 \mathrm{mo}, \\
6-9 \mathrm{mo} \text {, or } 9- \\
12 \mathrm{mo}, \mathrm{NSA}\end{array}$ \\
\hline \multirow[t]{4}{*}{$\begin{array}{l}\text { Huh, } 2011(37) \\
\text { U.S. } \\
\text { N: } 847\end{array}$} & $<4$ vs. $4-5 \mathrm{mo}, \mathrm{BF}$ & $\begin{array}{l}\text { Odds or prevalence of } \\
\text { obesity at age } 3 y: \text { NSA }\end{array}$ & $\begin{array}{l}\text { BMI at age } 3 y, \text { NSA; } \\
\text { BMIZ at age 3y, NSA; } \\
\text { Skinfolds at age 3y, } \\
\text { NSA }\end{array}$ & $\begin{array}{l}\text { Weight at age } \\
3 y, \text { NSA; WAZ } \\
\text { at 3y, NSA }\end{array}$ & $\begin{array}{l}\text { Height or HAZ at } \\
\text { age } 3 y, N S A\end{array}$ \\
\hline & $<4$ vs. $4-5 \mathrm{mo}, \mathrm{FF}$ & $\begin{array}{l}\text { Obesity at age } 3 y: \\
\text { OR=6.3; } 95 \% \mathrm{Cl}: 2.3,6.9, \\
\mathrm{P}<0.01 ; \\
\text { Prevalence of obesity, } \\
25.2 \% \text { vs. } 4.9 \% \\
\mathrm{P}<0.0001\end{array}$ & $\begin{array}{l}\text { BMIZ at age 3y: } \\
\beta=0.36 ; 95 \% \mathrm{Cl}: 0.10, \\
0.61, P=0.004 ; \\
\text { BMI at 3y: mean } 17.2 \text {, } \\
\text { SD:1.9 vs. } 16.5 \text {, } \\
\text { SD:1.5, } P=0.004 ; \\
\text { Skinfolds at age } 3 y \text { : } \\
\text { NSA }\end{array}$ & $\begin{array}{l}\text { Weight at age } \\
3 y: 16.7 \pm 2.7 \mathrm{~kg} \\
\text { vs. } 15.8 \pm 2.3 \mathrm{~g} \\
P=0.01 \\
\text { WAZ at } 3 \mathrm{y}: \\
0.92 \pm 1.1 \text { vs. } \\
0.55 \pm 0.9 \\
P=0.02\end{array}$ & $\begin{array}{l}\text { Height at age 3y, } \\
\text { NSA; HAZ at 3y, } \\
\text { NSA }\end{array}$ \\
\hline & $\geq 6$ vs. $4-5 \mathrm{mo}, \mathrm{BF}$ & $\begin{array}{l}\text { Odds or prevalence of } \\
\text { obesity at age } 3 y: \text { NSA }\end{array}$ & $\begin{array}{l}\text { BMIZ at age } 3 y, \text { NSA; } \\
\text { Skinfolds at age } 3 y \text {, } \\
\text { NSA }\end{array}$ & $\begin{array}{l}\text { Weight at age } \\
3 y, \text { NSA; WAZ } \\
\text { at } 3 y, N S A\end{array}$ & $\begin{array}{l}\text { Height at age } 3 y, \\
\text { NSA; HAZ at 3y, } \\
\text { NSA }\end{array}$ \\
\hline & $\geq 6$ vs. $4-5 \mathrm{mo}, \mathrm{FF}$ & $\begin{array}{l}\text { Odds of obesity at age } \\
3 y, N S A ; \\
\text { Prevalence of obesity, } \\
16 \% \text { vs. } 4.9 \%, P=0.06\end{array}$ & $\begin{array}{l}\text { BMIZ at age } 3 y, \text { NSA; } \\
\text { Skinfolds at age } 3 y \text {, } \\
\text { NSA }\end{array}$ & $\begin{array}{l}\text { Weight at age } \\
3 y: 16.6 \pm 2.7 \mathrm{~kg} \\
\text { vs. } 15.8 \pm 2.3 \mathrm{~kg}, \\
P=0.01 ; \text { WAZ, } \\
\text { NSA }\end{array}$ & $\begin{array}{l}\text { Height at age } 3 y \text {, } \\
\text { NSA; HAZ at 3y, } \\
\text { NSA }\end{array}$ \\
\hline
\end{tabular}




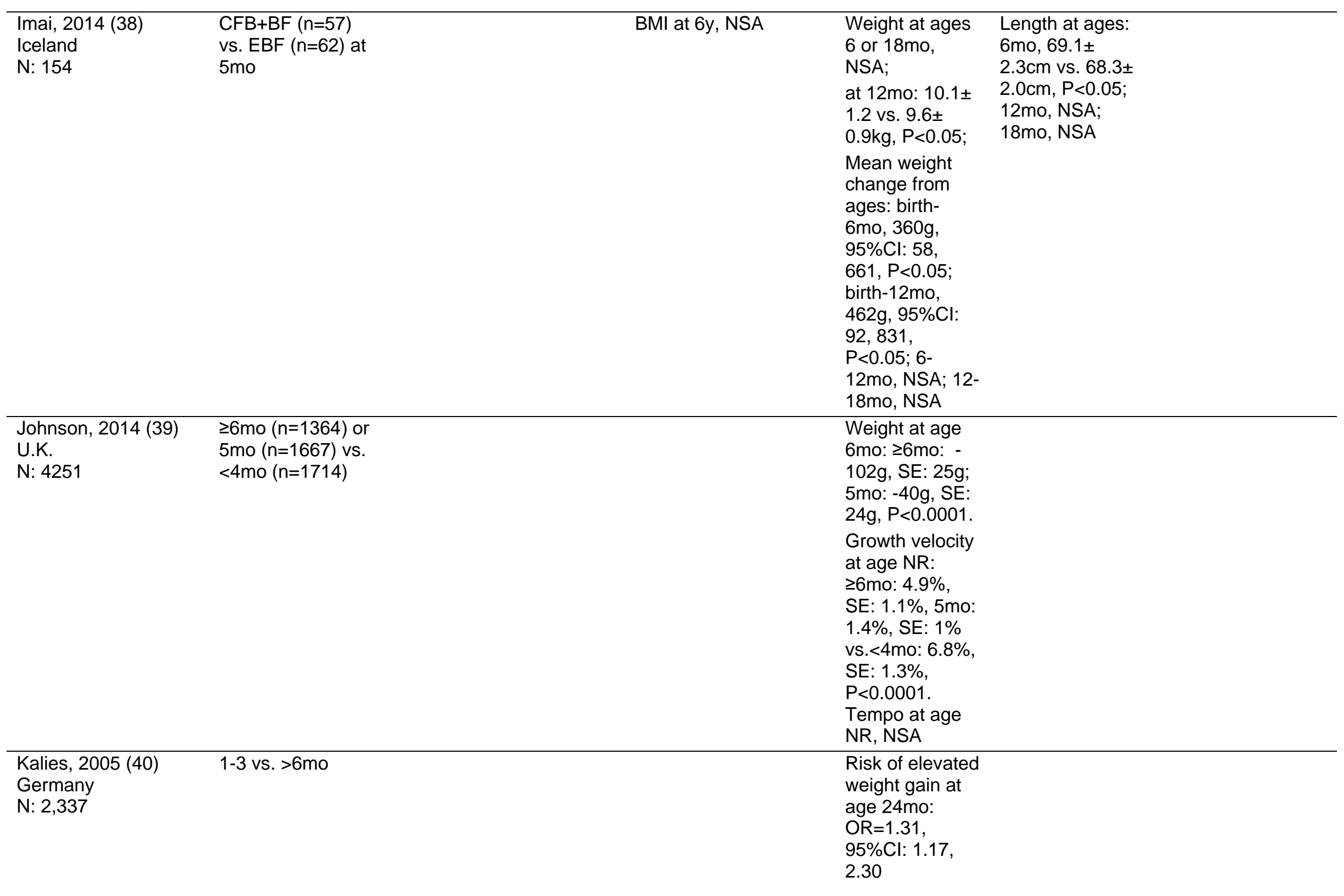


age $24 \mathrm{mo}$

$\mathrm{OR}=1.49$,

95\%Cl: 0.97,

\subsection{8}

\begin{tabular}{ll}
\hline Khadivzadeh, 2004 & CFB at 4mo vs \\
$(41)$ & EBF 4-6mo
\end{tabular}$$
\text { Iran }
$$

N: 193
Weigh

$5 \mathrm{mo}, \mathrm{NSA}$

6mo, NSA;

Weight gain

from 4-6mo,

NSA

Kramer, 1985 (43) Continuous
Canada

\section{Canada}

$\mathrm{N}: 347$

Kramer, 1985b Continuous

(42) Canada

N: 382

Kramer, 2004 (44)

\section{Belarus}

$\mathrm{N}: 17,046$

Skinfold thickness at age 24mo, NSA; BMI at age $24 \mathrm{mo}$, NSA

Skinfold thickness at
age $12 \mathrm{mo}, \beta=-0.141$, $r^{2}=0.038, P=0.002$;

$\mathrm{BMI}$ at $12 \mathrm{mo}$, NSA

Weight at age

24mo, NSA

\section{Weight at ages:}

$6 \mathrm{mo} \beta=-0.178$,

$\mathrm{P}<0.001 ; 12 \mathrm{mo}$

$\beta=-0.198$,

$\mathrm{P}<0.001$
Cereals at 3,6, or

9mo
WLZ from ages 3-6, 69, 9-12mo, NSA

WAZ from

ages: 3-6mo, $\beta=-0.293,95 \%$

Cl: -0.386, -

$0.199, \mathrm{P}=\mathrm{NR}$;

6-9mo, NSA; 9-

12mo, NSA

WLZ from ages 1-3, 3-

Other solids at 1 ,

3,6 , or $9 \mathrm{mo}$

6, 6-9, 9-12mo, NSA

WAZ from ages

1-3, 3-6, 6-9, 9-

$12 \mathrm{mo}, \mathrm{NSA}$

LAZ from ages:

3-6mo, $\beta=$

$0.240,95 \% \mathrm{Cl}:-$

$0.353,-0.127$,

$\mathrm{P}=\mathrm{NR} ; 6-9 \mathrm{mo}$

NSA; 9-12mo

NSA

LAZ from ages

1-3, 3-6, 6-9mo,

NSA; 9-12mo,

$\beta=-0.082,95 \%$

Cl: -0.156, -

$0.008, P=N R$

Juices/other liquids

WLZ from ages 1-3, 3- WAZ from ages

at $1,3,6$, or $9 \mathrm{mo}$
6, 6-9mo, NSA; 9-

$12 \mathrm{mo}, \beta=-0.061,95 \%$

1-3, 3-6, 6-9, 9 -

$12 \mathrm{mo}, \mathrm{NSA}$

Cl: $0.003,0.119$,

$\mathrm{P}=\mathrm{NR}$ 


\begin{tabular}{|c|c|c|c|c|c|}
\hline $\begin{array}{l}\text { Kuperberg, } 2006 \text { (45) } \\
\text { Canada } \\
\text { N: } 71\end{array}$ & $\begin{array}{l}\text { Continuous; <4mo } \\
\text { Y/N }\end{array}$ & $\begin{array}{l}\text { Odds of overweight at } \\
\text { age } 48 \mathrm{mo}, \mathrm{NSA}\end{array}$ & & & \\
\hline $\begin{array}{l}\text { Lande, } 2005(46) \\
\text { Norway } \\
\text { N: } 1441\end{array}$ & $\leq 4,4-5,>5 \mathrm{mo}$ & & $\begin{array}{l}\text { BMl at age } 12 \mathrm{mo} \text {, } \\
\text { NSA }\end{array}$ & & \\
\hline $\begin{array}{l}\text { Lauver, } 1981(47) \\
\text { U.S. } \\
\text { N: } 77\end{array}$ & $\leq 5$ or $>5 \mathrm{mo}$ & & & $\begin{array}{l}\text { Weight at age } \\
6 \mathrm{mo}, \mathrm{NSA}\end{array}$ & $\begin{array}{l}\text { Length at age } \\
6 \mathrm{mo}, \mathrm{NSA}\end{array}$ \\
\hline $\begin{array}{l}\text { Layte, } 2014(48) \\
\text { Ireland } \\
\mathrm{N}: 11,134\end{array}$ & $<4$ vs. $\geq 6 \mathrm{mo}$ & $\begin{array}{l}\text { Risk of obesity at age 3y, } \\
\text { NSA }\end{array}$ & & $\begin{array}{l}\text { Rapid weight } \\
\text { gain from age } \\
\text { 9mo-3y, NSA; } \\
\text { birth-9mo: } \\
\text { OR=1.5, } \\
P<0.001\end{array}$ & \\
\hline . & $<5$ vs. $\geq 6 \mathrm{mo}$ & $\begin{array}{l}\text { Risk of obesity at age 3y, } \\
\text { NSA }\end{array}$ & & $\begin{array}{l}\text { Rapid weight } \\
\text { gain from age } \\
\text { 9mo-3y, NSA; } \\
\text { birth-9mo: } \\
\text { OR=1.34, } \\
P=0.00\end{array}$ & \\
\hline . & $<6$ vs. $\geq 6 \mathrm{mo}$ & $\begin{array}{l}\text { Risk of obesity at age } 3 y \text {, } \\
\text { NSA }\end{array}$ & & $\begin{array}{l}\text { Rapid weight } \\
\text { gain from age } \\
\text { 9mo-3y, NSA; } \\
\text { birth-9mo: } \\
\text { OR=1.37, } \\
P=0.00\end{array}$ & \\
\hline $\begin{array}{l}\text { Leary, } 2015(49) \\
\text { U.K. } \\
\text { N: } 4,750\end{array}$ & 3 or $\geq 4$ vs. $\leq 2 \mathrm{mo}$ & & $\begin{array}{l}\text { Lean mass or FM at } \\
\text { age } 15 y, N S A\end{array}$ & & \\
\hline $\begin{array}{l}\text { Makela, } 2014(50) \\
\text { Finland } \\
\text { N: } 848\end{array}$ & Continuous, mo; & $\begin{array}{l}\text { Risk of overweight or } \\
\text { obesity at age } 24 \mathrm{mo} \text {, } \\
\text { NSA }\end{array}$ & & $\begin{array}{l}\text { Weight gain } \\
\text { from age birth } \\
\text { to } 24 \mathrm{mo}, \mathrm{NSA}\end{array}$ & \\
\hline & $<4,4-6,>6 \mathrm{mo}$ & & & $\begin{array}{l}\text { Weight gain, } \\
\text { from age birth- } \\
24 \mathrm{mo}, \mathrm{NSA}\end{array}$ & \\
\hline
\end{tabular}




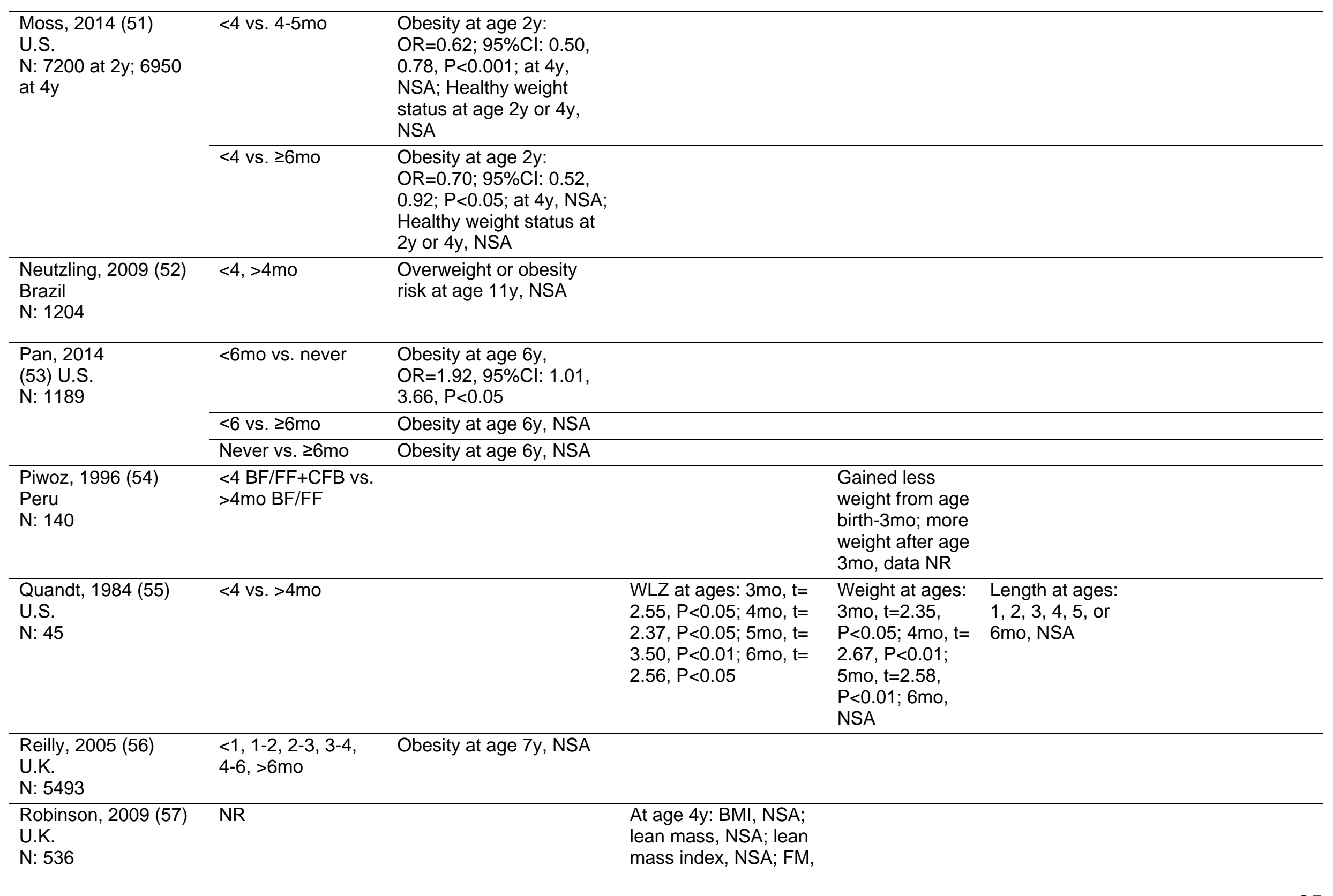




\begin{tabular}{|c|c|c|c|c|c|}
\hline $\begin{array}{l}\text { Rossiter, } 2013 \text { (58) } \\
\text { Canada } \\
\text { N: } 377\end{array}$ & $<4,>4 \mathrm{mo}$ & $\begin{array}{l}\text { Normal or underweight } \\
\text { vs. overweight or obesity } \\
\text { prevalence at age } 4 y \text {, } \\
\text { NSA }\end{array}$ & & & \\
\hline $\begin{array}{l}\text { Salmenpera, } 1985 \\
\text { (59) } \\
\text { Finland } \\
\mathrm{N}: 113 \text { at } 6-9 \mathrm{mo}, 84 \\
\text { at } 9-12 \mathrm{mo}\end{array}$ & $\begin{array}{l}\text { CFB }>6 \text { mo vs. } \\
\text { EBF } 6-9 \text { or } 9-12 \mathrm{mo}\end{array}$ & & & $\begin{array}{l}\text { Weight velocity } \\
\text { from ages 3-9, } \\
6-9,9-12 \text {, or } 6- \\
12 \mathrm{mo} \text {, all NSA }\end{array}$ & $\begin{array}{l}\text { Length velocity } \\
\text { from ages: } 3-9, \\
6-9 \mathrm{mo}, \mathrm{NSA} \text {, } 6- \\
12 \mathrm{mo}, 91.7, \mathrm{SE}: \\
1.8 \text { vs. } 89.3, \mathrm{SE}: \\
1.9, \mathrm{P}<0.05 ; 9- \\
12 \mathrm{mo}: 100.1 \\
\text { SE: } 2.0 \text { vs. } 87.5 \text {, } \\
\text { SE: } 7.7, \mathrm{P}<0.05\end{array}$ \\
\hline $\begin{array}{l}\text { Santorelli, } 2014 \text { (60) } \\
\text { U.K. } \\
\text { N: } 1327\end{array}$ & $<17$ vs. $>17 w k$ & & BMIZ at age $3 y, N S A$ & & \\
\hline \multirow[t]{2}{*}{$\begin{array}{l}\text { Schack-Nielsen, } \\
2010(61) \\
\text { Denmark } \\
\text { N: } 5068\end{array}$} & $\begin{array}{l}\text { Spoon-feeding: } \\
\text { continuous, mo }\end{array}$ & $\begin{array}{l}\text { At age } 42 y \text {, overweight or } \\
\text { obesity, NSA }\end{array}$ & $\begin{array}{l}\text { BMI ages: } 1-34 \mathrm{y}, \\
\text { NSA; } 42 \mathrm{y}, \beta=-0.046 \\
95 \% \mathrm{Cl}:-0.86,-0.006 \\
\mathrm{P}=0.03 ; \mathrm{WC} \text { at age } \\
42 \mathrm{y}, \beta=-0.25 \mathrm{~cm} / \mathrm{mo} ; \\
95 \% \mathrm{Cl}:-0.49,-0.01\end{array}$ & & \\
\hline & $\begin{array}{l}\text { Spoon-feeding, } \geq 4 \\
\text { vs }<4 \text { mo }\end{array}$ & $\begin{array}{l}\text { Lower risk of overweight } \\
\text { at age } 42 y, P=0.053\end{array}$ & & & \\
\hline $\begin{array}{l}\text { Seach, } 2010 \text { (62) } \\
\text { Australia } \\
\text { N: } 307\end{array}$ & Continuous, wk & $\begin{array}{l}\text { Risk of "above healthy" } \\
\text { BMl at age } 10 \mathrm{y}, \beta=0.903, \\
95 \% \mathrm{Cl}: 0.841,0.970 \\
\mathrm{P}=0.005\end{array}$ & & & \\
\hline $\begin{array}{l}\text { van Rossem, } 2013 \\
\text { (63) } \\
\text { Netherlands } \\
\text { N: } 3184\end{array}$ & $0-3,3-6,>6 \mathrm{mo}$ & & $\begin{array}{l}\text { WHZ change from age } \\
12-45 \mathrm{mo}, \mathrm{NSA}\end{array}$ & & \\
\hline
\end{tabular}




\begin{tabular}{|c|c|c|c|c|c|}
\hline $\begin{array}{l}\text { Victora, } 1998(64) \\
\text { Brazil } \\
\text { N: } 627\end{array}$ & $<3,>3 \mathrm{mo}$ & & $\begin{array}{l}\text { Ponderal index from } \\
\text { age 3-6mo, NSA }\end{array}$ & $\begin{array}{l}\text { Weight gain } \\
\text { from age 3- } \\
6 \mathrm{mo}, \mathrm{NSA}\end{array}$ & $\begin{array}{l}\text { Length gain from } \\
\text { age } 3-6 \mathrm{mo} \text {, NSA }\end{array}$ \\
\hline $\begin{array}{l}\text { Villalpando, } 2000 \\
(65) \\
\text { Mexico } \\
\text { N: } 170\end{array}$ & Continuous & & & $\begin{array}{l}\text { Weight gain } \\
\text { from age } 0- \\
6 \mathrm{mo},-104 \mathrm{~g}, \\
\text { SE: } 1.67, \\
P=0.03\end{array}$ & $\begin{array}{l}\text { Length gain from } \\
\text { age } 0-6 \mathrm{mo} \text {, NSA }\end{array}$ \\
\hline $\begin{array}{l}\text { Warrington, } 1988 \\
\text { (66) } \\
\text { U.K. } \\
\text { N: } 78\end{array}$ & Continuous, wk & & & $\begin{array}{l}\text { Weight at age } \\
1 y \text { or } 2 y, N S A\end{array}$ & \\
\hline $\begin{array}{l}\text { Wells, } 1998(67) \\
\text { U.K. } \\
\text { N: } 20\end{array}$ & $<12$ vs. $>12 w k$ & & $\begin{array}{l}\text { Skinfold thickness, FM } \\
\text { or FFM at age 2-3.5y, } \\
\text { NSA }\end{array}$ & $\begin{array}{l}\text { Weight at age } \\
2-3.5 y, N S A\end{array}$ & $\begin{array}{l}\text { Height at age 2- } \\
3.5 \mathrm{y}, \mathrm{NSA}\end{array}$ \\
\hline \multirow[t]{2}{*}{$\begin{array}{l}\text { Wen, 2014b (68) } \\
\text { Australia } \\
\text { N: } 242\end{array}$} & $3-5$ vs. $<2 \mathrm{mo}$ & $\begin{array}{l}\text { Overweight/obesity at } \\
\text { age } 2 y, O R=0.15, \\
95 \% C l: 0.03,0.55, \\
P<0.005\end{array}$ & $\mathrm{BMI}$ at age $2 \mathrm{y}, \mathrm{NSA}$ & & \\
\hline & $\geq 6$ vs. $<2 \mathrm{mo}$ & $\begin{array}{l}\text { Overweight/obesity at } \\
\text { age } 2 y, O R=0.14, \\
95 \% C l: 0.04,0.47, \\
P<0.005\end{array}$ & $\mathrm{BMI}$ at age $2 \mathrm{y}, \mathrm{NSA}$ & & \\
\hline $\begin{array}{l}\text { WHO, } 2002(69) \\
\text { China, India, } \\
\text { Guatemala, Nigeria, } \\
\text { Chile, Sweden, } \\
\text { Australia } \\
\text { N: } 1252\end{array}$ & $1-8$ vs. $17-24 w k$ & & & $\begin{array}{l}\text { Weight or } \\
\text { weight velocity } \\
\text { at age } 8 w k \text {, } \\
\text { NSA } \\
\text { Weight at } 24 w k \text {, } \\
49.6 \mathrm{~g}, \mathrm{SE}: \\
18.5, \mathrm{P}=0.028 \text {; } \\
\text { Weight velocity } \\
\text { at } 24 \mathrm{wk}, 3 \\
\mathrm{~g} / \mathrm{wk}, \mathrm{SE}: 1.17 \text {, } \\
\mathrm{P}=0.035\end{array}$ & $\begin{array}{l}\text { Length at age } \\
\text { 8wk, }-1.803 \mathrm{~mm} \text {, } \\
\mathrm{SE}: 0.311 \text {, } \\
\mathrm{P}=0.001 \text {; Length } \\
\text { velocity at age } \\
8 \mathrm{wk},-0.192 \\
\mathrm{~mm} / \mathrm{wk}, \mathrm{SE}: \\
0.08, P=0.049 ; \\
\text { Length or length } \\
\text { velocity at age } \\
\text { 24wk, NSA }\end{array}$ \\
\hline
\end{tabular}




\begin{tabular}{|c|c|c|c|c|}
\hline & $9-16$ vs $17-24 w k$ & & $\begin{array}{l}\text { Weight at age } \\
8 w k,-56.6 \mathrm{~g}, \\
\mathrm{SE}: 15, \\
\mathrm{P}=0.002 ; \\
\text { Weight velocity } \\
\text { at age } 8 \mathrm{wk},- \\
6.8 \mathrm{~g} / \mathrm{wk}, \mathrm{SE}: \\
2.55, \mathrm{P}=0.029 \text {; } \\
\text { Weight at age } \\
24 \mathrm{wk}, 51.6 \mathrm{~g}, \\
\mathrm{SE}: 16.8, \\
\mathrm{P}=0.012 ; \\
\text { Weight velocity } \\
\text { at age } 24 \mathrm{wk}, \\
\mathrm{NSA}\end{array}$ & $\begin{array}{l}\text { Length at age } \\
\text { 8wk, }-2.368 \mathrm{~mm} \text {, } \\
\mathrm{SE}: 0.278 \text {, } \\
\mathrm{P}=0.001 \text {; Length } \\
\text { velocity at age } \\
\text { 8wk, NSA. } \\
\text { Length at age } \\
24 \mathrm{wk}, 1.486 \mathrm{~mm} \text {, } \\
\mathrm{SE}: 0.283 \text {, } \\
\mathrm{P}=0.001 \text {; Length } \\
\text { velocity at age } \\
24 \mathrm{wk}, \mathrm{NSA}\end{array}$ \\
\hline & $25-32$ vs. $17-24 w k$ & & $\begin{array}{l}\text { Weight or } \\
\text { weight velocity } \\
\text { at ages 8wk or } \\
24 \mathrm{wk}, \mathrm{NSA}\end{array}$ & $\begin{array}{l}\text { Length at ages } \\
\text { 8wk or 24wk, } \\
\text { NSA; Length } \\
\text { velocity at ages: } \\
\text { 8wk, NSA; at } \\
24 w k,-0.151 \\
\mathrm{~mm} / \mathrm{wk}, \mathrm{SE}: \\
0.038, \mathrm{P}=0.001\end{array}$ \\
\hline $\begin{array}{l}\text { Wilson, } 1998 \text { (70) } \\
\text { U.K. } \\
\text { N: } 545\end{array}$ & $<15$ vs. $>15 w k$ & $\begin{array}{l}\text { \% body fat at age } 7 y, \\
18.5 \%, 95 \% \mathrm{Cl}: 18.2 \% \text {, } \\
18.8 \% \text { vs. } 16.5 \%, \\
95 \% \mathrm{Cl}: 16.0 \%, 17.0 \% \text {, } \\
\mathrm{P}<0.01 ; \mathrm{BMl} \text { at age } \\
7 \mathrm{y}, \mathrm{NSA}\end{array}$ & $\begin{array}{l}\text { Weight SD } \\
\text { score at age } 7 y \text {, } \\
0.02,95 \% \mathrm{Cl}:- \\
0.02,0.06 \text { vs. - } \\
0.09,95 \% \mathrm{Cl} \text { - } \\
0.16,0.02 \text {; } \\
\text { P<0.025 }\end{array}$ & $\begin{array}{l}\text { Height at age } 7 y \text {, } \\
\text { NSA }\end{array}$ \\
\hline $\begin{array}{l}\text { Wolman, } 1984 \text { (71) } \\
\text { U.S. } \\
\text { N: } 164\end{array}$ & $<12,>13 w k$ & $\mathrm{BMI}$ at age $4-6 \mathrm{y}, \mathrm{NSA}$ & $\begin{array}{l}\text { Weight at age4- } \\
6 y, \text { NSA }\end{array}$ & $\begin{array}{l}\text { Height at age 4- } \\
6 y, \text { NSA }\end{array}$ \\
\hline $\begin{array}{l}\text { Woo, } 2013(72) \\
\text { U.S., Mexico, China } \\
\text { N: } 285\end{array}$ & Continuous, mo & BMIZ at age $1 \mathrm{y}, \mathrm{NSA}$ & $\begin{array}{l}\text { WAZ at age } 1 y \text {, } \\
\text { NSA }\end{array}$ & $\begin{array}{l}\text { LAZ at age } 1 \mathrm{y}, \\
\text { NSA }\end{array}$ \\
\hline
\end{tabular}




Worobey, 2014 (74) Continuous, mo
U.S.
N: 154

N: 154

$<2$ vs. $>2$ mo

Canada

$\mathrm{N}: 316$

Zheng, 2015 (76) $\quad$ CFB, $\leq 3$ vs. 4-6mo
China

N: 40,510

\section{$6 \mathrm{mo}, \mathrm{NSA}$}

Weight gain

from age 3-

$6 \mathrm{mo}, \mathrm{NSA}$

\begin{tabular}{|c|c|c|c|}
\hline & \multicolumn{3}{|c|}{ age 4-5y, NSA } \\
\hline & CFB, 4-6 vs. $>6 \mathrm{mo}$ & $\begin{array}{l}\text { Overweight or obesity at } \\
\text { age } 4-5 y, N S A\end{array}$ & BMI at age $4-5 y, N S A$ \\
\hline \multicolumn{4}{|l|}{$\begin{array}{l}\text { Retrospective } \\
\text { cohort studies }\end{array}$} \\
\hline $\begin{array}{l}\text { Burdette, } 2006 \text { (77) } \\
\text { U.S. } \\
\text { N: } 313\end{array}$ & $\begin{array}{l}\text { No solids }<4 \mathrm{mo}+ \\
\text { no sugar- } \\
\text { sweetened } \\
\text { beverages }<6 \mathrm{mo} \\
\text { vs. not }\end{array}$ & $\begin{array}{l}\text { Prevalence of overweight } \\
\text { at age } 5 y, N S A\end{array}$ & $\begin{array}{l}\text { Mean FM at age } 5 y \text {, } \\
\text { high \% body fat at age } \\
5 y ; \text { BMIZ at } 5 y, \text { NSA }\end{array}$ \\
\hline
\end{tabular}

\section{Nested case-}

control/case-control

\section{studies}

\begin{tabular}{ll}
\hline Bammann, 2014 & $<4 \mathrm{mo}$ \\
(78), Nested case- & Obesity risk at age 2- \\
control; Belgium, & $9 y$, NSA \\
Cyprus, Estonia, & \\
Germany, Hungary, & \\
Italy, Spain and & \\
Sweden & \\
N: 1024 & \\
\hline
\end{tabular}

Sum of skinfold

thickness at age $6 \mathrm{mo}$,

NSA

BMI at age 4-5y,

$\begin{array}{ll}\text { Overweight at age 4-5y, } & \mathrm{BMI} \text { at ag } \\ \text { OR }=1.11 ; 95 \% \mathrm{Cl}: 1.03, & \mathrm{P}<0.001\end{array}$

1.19; $P=0.038$; Obesity at

age $4-5 y$, NSA

Overweight or obesity at 


\begin{tabular}{|c|c|c|}
\hline $\begin{array}{l}\text { Gungor, } 2010 \\
\text { (79) U.S. } \\
\text { N: } 129\end{array}$ & $\begin{array}{l}\text { Continuous, mo in } \\
\text { non-overweight vs. } \\
\text { overweight at 6-8y }\end{array}$ & $\begin{array}{l}\text { Mean } 6.49, \text { SD: } 2.80 \mathrm{mo} \\
\text { vs. } 5.10 \text { SD: } 1.80 \mathrm{mo}, \\
P=0.007\end{array}$ \\
\hline $\begin{array}{l}\text { Hui, } 2003(80) \\
\text { China } \\
\text { N: } 343\end{array}$ & $<4 \mathrm{mo} \mathrm{Y} / \mathrm{N}$ & $\begin{array}{l}\text { Prevalence of } \\
\text { overweight, normal- } \\
\text { middle weight, or normal- } \\
\text { low weight at age } 6-7 y \text {, } \\
\text { NSA }\end{array}$ \\
\hline \multirow[t]{2}{*}{$\begin{array}{l}\text { Zhou, } 2011(81) \\
\text { China } \\
\text { N: } 162\end{array}$} & $<4$ vs. $4-6 m o$ & $\begin{array}{l}\text { Obesity at age } 3-6 y \\
\text { OR=10.96; } 95 \% \mathrm{Cl}: 2.08 \text {, } \\
21.64, \mathrm{P}=0.007\end{array}$ \\
\hline & $4-6$ vs. $>6 \mathrm{mo}$ & $\begin{array}{l}\text { Obesity risk at age 3-6y, } \\
\text { NSA }\end{array}$ \\
\hline
\end{tabular}

1. The independent variable/exposure of interest included timing of CFB introduction.

2. Outcomes were categorized in accordance with the analytical framework (also see Figure 1). Results were extracted from multivariate-adjusted analyses wherever possible rather than univariate analyses, crude, and/or unadjusted measures. Standard deviation (SD) or standard error of the mean (SE) are indicated.

Abbreviations: CFB, complementary food/beverage; BF, breast-fed; FF, formula-fed; FM, fat mass; FFM, fat-free mass; FMI, fat mass index; FFMI, fat-free mass index; FMZ, fat mass z-score, FFMZ, fat-free mass z-score; NR, not reported; NSA, no significant associations; NSGD, no significant group differences; $\mathrm{SD}$, standard deviation; SE, standard error of the mean 


\section{Discussion}

Moderate evidence suggests that first introduction of any CFB between 4-5 mo compared to approximately 6 mo of age is not associated with differences in weight status, body composition, body circumferences, weight or length among generally healthy, full-term infants. Limited evidence suggests that introducing CFB before 4 mo of age may be associated with higher odds of overweight/obesity. There is not enough evidence to determine the relationship between introduction of CFB at $>7 \mathrm{mo}$ of age on growth, size, or body composition.

With the exception of the five included articles from two RCTs, which have a stronger study design relative to the observational studies, little evidence was provided to permit evaluation of causal relationships between timing of CFB introduction and growth, size, body composition, and/or weight status. The majority of included evidence came from prospective cohort studies (71 of 81 articles). Authors of three articles (both RCTs) noted limitations regarding sample size (1-3). Many of the observational studies (35 of 75 ) did not account for high rates of attrition/missing data but some did have large sample sizes to investigate the relationship between timing of introduction to CFB and growth, size, body composition, and/or weight status.

The majority of studies included in this SR were consistent across the body of evidence in terms of direction and statistical significance of findings, reporting no significant association between timing of CFB introduction and outcomes in terms of weight status (14 of 28 articles), body composition (adiposity: 14 of 19 articles, weightto-height: 19 of 29 articles, and central adiposity: 4 of 5 articles), weight and length/height (18 of 28 articles), and/or head, arm, or thigh circumferences (7 of 8 articles). The evidence is strongest for studies examining introduction of CFB at approximately age 4 mo compared to 6 mo, which includes two RCTs (1-5). For the remaining observational studies that make up the body of evidence, there were considerable methodological limitations and inconsistencies in both the magnitude of observed effects and direction of findings. There was sufficient consistency among the observational studies reporting higher odds of overweight/obesity or weight when comparing very early $(<4 \mathrm{mo})$ relative to later CFB introduction (various time points), such that a limited conclusion was warranted. Few studies examining growth, size, and/or body composition made an attempt to more specifically differentiate introduction at $4-5$ vs. 6 mo of age. Combining participants into categories such as CFB $\geq 6$ mo made comparisons across the body of evidence difficult, such that no conclusion was drawn regarding the introduction of CFB at age 7 mo or older. There was also inconsistency across studies in the body of evidence with respect to several factors: milk-feeding practices, the specific type of CFB that were introduced, the specific age interval when CFB were introduced, which outcomes were considered, and the age at which the outcome was measured.

Most studies in this SR directly examined timing of introduction of CFB and the outcomes of interest but varied in practical/clinical significance. Consideration of a broader-spectrum of growth-related outcomes than were included in many of the studies in this body of evidence may have led to stronger conclusions. For example, studies that examined weight status tended to report only weight status and not multiple outcomes, which would have provided important contextual information to aid 
in the interpretation of weight status results. Any relationships observed within this body of evidence must be interpreted with caution, as earlier (i.e., human milk/infant formula) and later (toddler) feeding practices associated with CFB introduction may have stronger influences on growth, size, body composition, and/or weight status.

Overall, there was good generalizability with respect to the U.S. population. However, several studies were conducted in populations that were less similar to the U.S. population. For example, studies conducted in Israel, Peru, and Brazil enrolled participants, who either migrated from other countries with lower HDI or were from very disadvantaged populations $(22,54,64,68)$. In addition, some studies were less relevant to the U.S. due to differences in complementary feeding practices at the time the study was conducted and/or because they examined specific CFB that are less prominent as typical CFB in the U.S $(15,61,76)$.

\section{Limitations}

A primary methodological limitation across the observational studies was the variability in controlling for confounding factors. The majority of observational studies did not account for more than one of the key confounders from the analytic framework. Of the studies that identified at least one significant association, many did not adjust for baseline anthropometric values and either adjusted for birth weight or reported anthropometric outcomes at multiple time points. The majority of articles $(\sim 80 \%)$ adjusted for feeding practices aside from complementary feeding but how feeding practices were accounted for varied across studies. For example, studies may have enrolled either BF or FF infants, enrolled both but analyzed BF and FF infants separately, adjusted for duration of BF or FF in analyses, or did not adjust analyses but reported no differences between groups at baseline. There was minor inconsistency across studies in the description of human milk, infant formula, and/or types and amounts of CFB consumed. The majority of studies commonly defined CFB as solids other than human milk or infant formula, but several studies also reported the age of introduction of specific types of CFB $(9,11,15,18,19,44,60,61,76)$, which are discussed in more detail in a separate SR (83).

Another limitation is the limited reliability/validity of assessment methods for both timing of CFB introduction and outcome assessment. Several studies used reported, not measured, weight/height from various sources $(11,28,39,47,61,71)$. Several studies did not describe their methods to assess outcomes $(1,33)$ or used unstandardized methods (44). Although the majority of observational studies reported using "standard procedures" for anthropometric measurements, many of the studies either lacked sufficient description of outcome assessment or indicated that measures were only collected once as opposed to in duplicate or triplicate $(6,8,13,22,26,31$ $34,36-38,40,42-44,46,48,50,53,55,59,64-67,70,76,81)$. Another limitation common to the body of evidence was the assessment of timing of CFB introduction. Two studies did not clearly define their methods for analyzing timing of CFB introduction $(27,57)$. Multiple studies had distinct or unique ages at which they examined timing of CFB introduction (for examples see $25,54,69,79$ ), which does not allow for interpretation based on time points outside of those distinct or unique ages. Finally, many of the included articles examined CFB introduction $<4$ mo of age as the earliest category of introduction, with fewer relative comparison groups between 4-6 
mo of age and/or $>6$ mo of age.

Finally, internal validity was a concern in several studies. In both of the RCTs, blinding of investigators could not be determined and participants were not blinded as they either received CFB or remained exclusively BF/FF. In the majority of the observational studies, blinding of outcome assessors could not be determined. Adequacy of statistical methods in several studies was insufficient due to either a lack of detail describing data or inconsistency of data reported $(5,16,47,54,66)$ or difficulty in interpretation based on time points reported (69). 


\section{Research recommendations}

- Future research should account for the substantial individual variability that is likely in terms of the ideal timing of CFB introduction, which may contribute to the relationship between timing of CFB and growth, size, body composition, and/or weight status in a given study population. Factors to consider include:

- Parent rationale for timing of introduction of CFB (e.g., infant feeding style, infant demand, infant size/growth faltering, attitudes/beliefs, fear of inadequate milk supply, infant sleep promotion)

- Plasticity across time (intrauterine, genetic, and/or environmental effects)

- Predominant type of early milk feeding (e.g. exclusive breastfeeding or formula feeding or mixed)

- In studies examining the association between timing of CFB introduction and child growth, inclusion of multiple growth-related measures substantially aid interpretation of results.

- Observational studies should be a low priority, in particular those that examine only one outcome and/or categorize by CFB introduction before $4 \mathrm{mo}$ of age, in favor of additional rigorous RCTs that examine multiple outcomes and/or CFB introduction between the ages of 4 mo and 6 mo or later.

\section{Included articles}

1. Bainbridge RR, Mimouni FB, Landi T, Crossman M, Harris L, Tsang RC. Effect of rice cereal feedings on bone mineralization and calcium homeostasis in cow milk formula fed infants. Journal of the American College of Nutrition. 1996;15:383-8.

2. Jonsdottir, $\mathrm{OH}$, Kleinman, $\mathrm{R} \mathrm{E}$, Wells, J C, et al. Exclusive breastfeeding for 4 versus 6 months and growth in early childhood. Acta paediatrica (Oslo, Norway : 1992). 2014;103(1):105-11. 3. Jonsdottir, O H, Thorsdottir, I, Gunnlaugsson, G, et al. Exclusive breastfeeding and developmental and behavioral status in early childhood. Nutrients. 2013;5(11):4414-28.

4. Jonsdottir, $\mathrm{O} \mathrm{H}$, Thorsdottir, I, Hibberd, P L, et al. Timing of the introduction of complementary foods in infancy: a randomized controlled trial. Pediatrics. 2012;130(6):1038-45.

5. Wells, J C, Jonsdottir, O H, Hibberd, P L, et al. Randomized controlled trial of 4 compared with 6 mo of exclusive breastfeeding in Iceland: differences in breast-milk intake by stable-isotope probe. The American journal of clinical nutrition. 2012;96(1):73-9.

6. Abraham, E C, Godwin, J, Sherriff, A, et al. Infant feeding in relation to eating patterns in the second year of life and weight status in the fourth year. Public health nutrition. 2012;15(9):1705-14.

7. Agras, W S, Kraemer, H C, Berkowitz, R I, et al. Influence of early feeding style on adiposity at 6 years of age. The Journal of pediatrics. 1990;116(5):805-9.

8. Atkins, L A, McNaughton, S A, Campbell, $\mathrm{K} \mathrm{J}$, et al. Iron intakes of Australian infants and toddlers: Findings from the Melbourne Infant Feeding, Activity and Nutrition Trial (InFANT) Program. British Journal of Nutrition. 2016;115(2):285-93.

9. Ay, L, Hokken K, A C, Mook K, D O, et al. Tracking and determinants of subcutaneous fat mass in early childhood: the Generation R Study. International journal of obesity (2005). 2008;32(7):1050-9.

10. Baird, J, Poole, J, Robinson, S, et al. Milk feeding and dietary patterns predict weight and fat gains in infancy. Paediatric and perinatal epidemiology. 2008;22(6):575-86. 
11. Baker, J L, Michaelsen, K F, Rasmussen, K M, et al. Maternal prepregnant body mass index, duration of breastfeeding, and timing of complementary food introduction are associated with infant weight gain. The American journal of clinical nutrition. 2004;80(6):1579-88.

12. Barrera, C M, Perrine, C G, Li, R, et al. Age at Introduction to Solid Foods and Child Obesity at 6 Years. Childhood obesity (Print). 2016;12(3):188-92.

13. Barton, S J, Howard, P K, Rayens, M K. The effects of infant feeding decisions on infant growth. Journal for specialists in pediatric nursing : JSPN. 2002;7(2):64-70.

14. Butte NF, Wong WW, Hopkinson JM, Smith EO, Ellis KJ. Infant feeding mode affects early growth and body composition. Pediatrics. 2000;106:1355-66.

15. Cantoral, A, Tellez R, M M, Ettinger, A S, et al. Early introduction and cumulative consumption of sugar-sweetened beverages during the pre-school period and risk of obesity at 8-14 years of age. Pediatric obesity. 2016;11(1):68-74.

16. Carruth BR, Skinner JD, Houck KS, Moran JD. Addition of supplementary foods and infant growth (2 to 24 months). Journal of the American College of Nutrition. 2000;19:405-12.

17. de Beer M, Vrijkotte TG, Fall CH, van Eijsden M, Osmond C, Gemke RJ. Associations of infant feeding and timing of linear growth and relative weight gain during early life with childhood body composition. International journal of obesity (2005). 2015;39:586-92.

18. Durmus, B, Heppe, D H, Gishti, O, et al. General and abdominal fat outcomes in school-age children associated with infant breastfeeding patterns. The American journal of clinical nutrition. 2014;99(6):1351-8.

19. Durmus B, Ay L, Duijts L, Moll HA, Hokken-Koelega AC, Raat H, et al. Infant diet and subcutaneous fat mass in early childhood: the Generation R Study. European journal of clinical nutrition. 2012;66:253-60.

20. Ejlerskov, K T, Christensen, L B, Ritz, C, et al. The impact of early growth patterns and infant feeding on body composition at 3 years of age. The British journal of nutrition. 2015;114(2):316-27. 21. Fairley, L, Santorelli, G, Lawlor, D A, et al. The relationship between early life modifiable risk factors for childhood obesity, ethnicity and body mass index at age 3 years: findings from the Born in Bradford birth cohort study. BMC obesity. 2015;2:9.

22. Fawzi, W W, Forman, M R, Levy, A, et al. Maternal anthropometry and infant feeding practices in Israel in relation to growth in infancy: the North African Infant Feeding Study. The American journal of clinical nutrition. 1997;65(6):1731-7.

23. Ferris, A G, Laus, M J, Hosmer, D W, et al. The effect of diet on weight gain in infancy. The American journal of clinical nutrition. 1980;33(12):2635-42.

24. Flores, G, Lin, H. Factors predicting overweight in US kindergartners. The American journal of clinical nutrition. 2013;97(6):1178-87.

25. Flores $\mathrm{G}$, Lin $\mathrm{H}$. Factors predicting severe childhood obesity in kindergarteners. International journal of obesity (2005). 2013;37(1):31-9.

26. Forsyth, J S, Ogston, S A, Clark, A, et al. Relation between early introduction of solid food to infants and their weight and illnesses during the first two years of life. BMJ (Clinical research ed). 1993;306(6892):1572-6.

27. Friel, J K, Gibson, R S, Kawash, G F, et al. Dietary zinc intake and growth during infancy. Journal of pediatric gastroenterology and nutrition. 1985;4(5):746-51.

28. Gaffney, K F, Kitsantas, P, Cheema, J. Clinical practice guidelines for feeding behaviors and weight-for-age at 12 months: a secondary analysis of the Infant Feeding Practices Study II. Worldviews on evidence-based nursing / Sigma Theta Tau International, Honor Society of Nursing. 2012;9(4):234-42.

29. Garden, F L, Marks, G B, Simpson, J M, et al. Body mass index (BMI) trajectories from birth to 11.5 years: relation to early life food intake. Nutrients. 2012;4(10):1382-98. 
30. Gibbs, B G, Forste, R. Socioeconomic status, infant feeding practices and early childhood obesity. Pediatric obesity. 2014;9(2):135-46.

31. Griffiths, L J, Smeeth, L, Hawkins, S S, et al. Effects of infant feeding practice on weight gain from birth to 3 years. Archives of disease in childhood. 2009;94(8):577-82.

32. Griffiths LJ, Hawkins SS, Cole TJ, Dezateux C. Risk factors for rapid weight gain in preschool children: findings from a UK-wide prospective study. International journal of obesity (2005). 2010;34(4):624-32.

33. Grote, V, Schiess, S A, Closa M, R, et al. The introduction of solid food and growth in the first 2 $\mathrm{y}$ of life in formula-fed children: analysis of data from a European cohort study. The American journal of clinical nutrition. 2011;94(6 Suppl):1785s-93s.

34. Haschke, F, van't H, M A. Euro-Growth references for breast-fed boys and girls: influence of breast-feeding and solids on growth until 36 months of age. Euro-Growth Study Group. Journal of pediatric gastroenterology and nutrition. 2000;31 Suppl 1:S60-71.

35. Hawkins SS, Cole TJ, Law C. An ecological systems approach to examining risk factors for early childhood overweight: findings from the UK Millennium Cohort Study. Journal of epidemiology and community health. 2009;63:147-55.

36. Heinig, M J, Nommsen, L A, Peerson, J M, et al. Intake and growth of breast-fed and formulafed infants in relation to the timing of introduction of complementary foods: the DARLING study. Davis Area Research on Lactation, Infant Nutrition and Growth. Acta paediatrica (Oslo, Norway : 1992). 1993;82(12):999-1006.

37. Huh, S Y, Rifas S, S L, Taveras, E M, et al. Timing of solid food introduction and risk of obesity in preschool-aged children. Pediatrics. 2011;127(3):e544-51.

38. Imai, C M, Gunnarsdottir, I, Thorisdottir, B, et al. Associations between infant feeding practice prior to six months and body mass index at six years of age. Nutrients. 2014;6(4):1608-17.

39. Johnson, L, van J, C H, Llewellyn, $\mathrm{C} \mathrm{H}$, et al. Associations between infant feeding and the size, tempo and velocity of infant weight gain: SITAR analysis of the Gemini twin birth cohort. International journal of obesity (2005). 2014;38(7):980-7.

40. Kalies, H, Heinrich, J, Borte, N, et al. The effect of breastfeeding on weight gain in infants: results of a birth cohort study. European journal of medical research. 2005;10(1):36-42.

41. Khadivzadeh, T, Parsai, S. Effect of exclusive breastfeeding and complementary feeding on infant growth and morbidity. Eastern Mediterranean health journal = La revue de sante de la Mediterranee orientale = al-Majallah al-sihhiyah li-sharq al-mutawassit. 2004;10(3):289-94 .

42. Kramer, M S, Barr, R G, Leduc, D G, et al. Determinants of weight and adiposity in the first year of life. The Journal of pediatrics. 1985;106(1):10-4.

43. Kramer, M S, Barr, R G, Leduc, D G, et al. Infant determinants of childhood weight and adiposity. The Journal of pediatrics. 1985;107(1):104-7.

44. Kramer, M S, Guo, T, Platt, R W, et al. Feeding effects on growth during infancy. The Journal of pediatrics. 2004;145(5):600-5.

45. Kuperberg, K, Evers, S. Feeding patterns and weight among First Nations children. Canadian journal of dietetic practice and research : a publication of Dietitians of Canada = Revue canadienne de la pratique et de la recherche en dietetique : une publication des Dietetistes du Canada.

2006;67(2):79-84.

46. Lande, B, Andersen, L F, Henriksen, T, et al. Relations between high ponderal index at birth, feeding practices and body mass index in infancy. European journal of clinical nutrition. 2005;59(11):1241-9.

47. Lauver, M A, Hizon, L, Bulla, A, et al. Infant feeding practices: the effect on six month weight. The Journal of the Kansas Medical Society. 1981;82(9):403-6. 
48. Layte, R, Bennett, A, McCrory, C, et al. Social class variation in the predictors of rapid growth in infancy and obesity at age 3 years. International journal of obesity (2005). 2014;38(1):82-90.

49. Leary, S D, Lawlor, D A, Davey S, G, et al. Behavioural early-life exposures and body composition at age 15 years. Nutrition \& diabetes. 2015;5:e150.

50. Makela J, Vaarno J, Kaljonen A, Niinikoski H, Lagstrom H. Maternal overweight impacts infant feeding patterns--the STEPS Study. European journal of clinical nutrition. 2014;68:43-9.

51. Moss, B G, Yeaton, W H. Early childhood healthy and obese weight status: potentially protective benefits of breastfeeding and delaying solid foods. Maternal and child health journal. 2014;18(5):1224-32.

52. Neutzling, M B, Hallal, P R, Araujo, C L, et al. Infant feeding and obesity at 11 years: prospective birth cohort study. International journal of pediatric obesity : IJPO : an official journal of the International Association for the Study of Obesity. 2009;4(3):143-9.

53. Pan L, Li R, Park S, Galuska DA, Sherry B, Freedman DS. A longitudinal analysis of sugarsweetened beverage intake in infancy and obesity at 6 years. Pediatrics. 2014;134 Suppl 1:S29-35. 54. Piwoz, E G, Creed de K, H, Lopez de R, G L, et al. Feeding practices and growth among lowincome Peruvian infants: a comparison of internationally-recommended definitions. International journal of epidemiology. 1996;25(1):103-14.

55. Quandt, S A. The effect of beikost on the diet of breast-fed infants. Journal of the American Dietetic Association. 1984;84(1):47-51.

56. Reilly, J J, Armstrong, J, Dorosty, A R, et al. Early life risk factors for obesity in childhood: cohort study. BMJ (Clinical research ed). 2005;330(7504):1357.

57. Robinson SM, Marriott LD, Crozier SR, Harvey NC, Gale CR, Inskip HM, et al. Variations in infant feeding practice are associated with body composition in childhood: a prospective cohort study. The Journal of clinical endocrinology and metabolism. 2009;94:2799-805.

58. Rossiter, M D, Evers, S E. Infant feeding practices and children's weight status. Canadian journal of dietetic practice and research : a publication of Dietitians of Canada $=$ Revue canadienne de la pratique et de la recherche en dietetique : une publication des Dietetistes du Canada.

2013;74(3):107-13.

59. Salmenpera, L, Perheentupa, J, Siimes, M A. Exclusively breast-fed healthy infants grow slower than reference infants. Pediatric research. 1985;19(3):307-12.

60. Santorelli, G, Fairley, L, Petherick, E S, et al. Ethnic differences in infant feeding practices and their relationship with BMI at 3 years of age - results from the Born in Bradford birth cohort study. The British journal of nutrition. 2014;111(10):1891-7.

61. Schack-Nielsen L, Sorensen T, Mortensen EL, Michaelsen KF. Late introduction of complementary feeding, rather than duration of breastfeeding, may protect against adult overweight. The American journal of clinical nutrition. 2010;91:619-27.

62. Seach, K A, Dharmage, S C, Lowe, A J, et al. Delayed introduction of solid feeding reduces child overweight and obesity at 10 years. International journal of obesity (2005). 2010;34(10):1475-9. 63. van Rossem L, Kiefte-de Jong JC, Looman CW, Jaddoe VW, Hofman A, Hokken-Koelega AC, et al. Weight change before and after the introduction of solids: results from a longitudinal birth cohort. The British journal of nutrition. 2013;109:370-5.

64. Victora, C G, Morris, S S, Barros, F C, et al. Breast-feeding and growth in Brazilian infants. The American journal of clinical nutrition. 1998;67(3):452-8.

65. Villalpando, S, Lopez A, M. Growth faltering is prevented by breast-feeding in underprivileged infants from Mexico City. The Journal of nutrition. 2000;130(3):546-52.

66. Warrington, S, Storey, D M. Comparative studies on Asian and Caucasian children. 2:

Nutrition, feeding practices and health. European journal of clinical nutrition. 1988;42(1):69-79. 
67. Wells, J C, Stanley, M, Laidlaw, A S, et al. Energy intake in early infancy and childhood fatness. International journal of obesity and related metabolic disorders : journal of the International Association for the Study of Obesity. 1998;22(5):387-92.

68. Wen, L M, Baur, L A, Rissel, C, et al. Correlates of body mass index and overweight and obesity of children aged 2 years: findings from the healthy beginnings trial. Obesity (Silver Spring, Md). 2014;22(7):1723-30.

69. World Health Organization. Growth of healthy infants and the timing, type, and frequency of complementary foods. The American journal of clinical nutrition. 2002;76(3):620-7.

70. Wilson, A C, Forsyth, J S, Greene, S A, et al. Relation of infant diet to childhood health: seven year follow up of cohort of children in Dundee infant feeding study. BMJ (Clinical research ed). 1998;316(7124):21-5.

71. Wolman, P G. Feeding practices in infancy and prevalence of obesity in preschool children. Journal of the American Dietetic Association. 1984;84(4):436-8.

72. Woo, J G, Guerrero, M L, Ruiz P, G M, et al. Specific infant feeding practices do not consistently explain variation in anthropometry at age 1 year in urban United States, Mexico, and China cohorts. The Journal of nutrition. 2013;143(2):166-74.

73. Worobey, J, Lopez, M I, Hoffman, D J. Maternal behavior and infant weight gain in the first year. Journal of nutrition education and behavior. 2009;41(3):169-75.

74. Worobey J, Pena J, Ramos I, Espinosa C. Infant difficulty and early weight gain: does fussing promote overfeeding? Maternal \& child nutrition. 2014;10:295-303.

75. Yeung, D L, Pennell, M D, Leung, M, et al. Infant fatness and feeding practices: a longitudinal assessment. Journal of the American Dietetic Association. 1981;79(5):531-5.

76. Zheng, J S, Liu, H, Zhao, Y M, et al. Complementary feeding and childhood adiposity in preschool-aged children in a large Chinese cohort. The Journal of pediatrics. 2015;166(2):326-31.e2. 77. Burdette, H L, Whitaker, R C, Hall, W C, et al. Breastfeeding, introduction of complementary foods, and adiposity at $5 \mathrm{y}$ of age. The American journal of clinical nutrition. 2006;83(3):550-8. 78. Bammann K, Peplies J, De Henauw S, Hunsberger M, Molnar D, Moreno LA, et al. Early life course risk factors for childhood obesity: the IDEFICS case-control study. PloS one. 2014;9(2):e86914.

79. Gungor DE, Paul IM, Birch LL, Bartok CJ. Risky vs rapid growth in infancy: refining pediatric screening for childhood overweight. Archives of pediatrics \& adolescent medicine. 2010;164:1091-7. 80. Hui, L L, Nelson, E A, Yu, L M, et al. Risk factors for childhood overweight in 6- to 7-y-old Hong Kong children. International journal of obesity and related metabolic disorders : journal of the International Association for the Study of Obesity. 2003;27(11):1411-8.

81. Zhou, L, He, G, Zhang, J, et al. Risk factors of obesity in preschool children in an urban area in China. European journal of pediatrics. 2011;170(11):1401-6.

\section{Other references}

82. United Nations Development Programme. Human Development Report 2014. Sustaining Human Progress: Reducing Vulnerabilities and Building Resilience. New York; 2014.

83. English L, Obbagy, JE, Wong, YP, Butte, NF, Dewey, KG, Fox, MK, Greer, FR, Krebs, NF, Scanlon, KS, Stoody, EE. Timing of introduction of complementary foods and beverages and growth, size, and body composition: A systematic review. Amer J Clin Nutr. 2019;109(7):935S-55S.

84. World Bank. The Little Data Book 2016 Washington, DC2016 [Available from:

https://openknowledge.worldbank.org/handle/10986/23968. 


\section{ANALYTIC FRAMEWORK}

The analytic framework (Figure 1) illustrates the overall scope of the systematic review, including the population, the interventions and/or exposures, comparators, and outcomes of interest. It also includes definitions of key terms and identifies key confounders considered in the systematic review. This is the analytic framework for the systematic review conducted to examine the relationship between complementary feeding and growth, size, and body composition.

\section{Figure 1: Analytic framework}

Analytic framework

- What is the relationship between timing of introduction of complementary foods and beverages and growth, size, and body composition?

\begin{tabular}{|c|c|}
\hline \multicolumn{2}{|l|}{ Target Population } \\
\hline \multicolumn{2}{|c|}{$\begin{array}{l}\text { Generally healthy infants fed human milk, infant formula, or both, } \\
\text { with growth, size, and body composition examined through } \\
\text { adulthood }\end{array}$} \\
\hline & $\downarrow$ \\
\hline Intervention/Exposure & Comparator \\
\hline $\begin{array}{l}\text { Timing of introduction of } \\
\text { complementary foods } \\
\text { and beverages (CFB) }\end{array}$ & $\begin{array}{l}\text { Different timing of introduction } \\
\text { of CFB }\end{array}$ \\
\hline \multicolumn{2}{|l|}{ Health Outcomes } \\
\hline $\begin{array}{l}\text { - Weight and length/height } \\
\text { - BMI, BMI percentile for ag } \\
\text { - Waist circumference } \\
\text { - Weight change } \\
\text { - Weight status change } \\
\text { height-for-age, length/sta } \\
\text { - Change across more than } \\
\text { length-for-age, weight-for-I } \\
\text { circumference for age } \\
\text { - \% fat mass, \% fat free ma } \\
\text { - Skinfold thickness } \\
\text { - Incidence of healthy weigh } \\
\text { - Incidence of underweight } \\
\text { wasting in infants and chil }\end{array}$ & $\begin{array}{l}\text { and } z \text {-score } \\
\text { ure-for-age, weight-for-length, } \\
\text { nference for age } \\
\text { one time point of weight-for age, } \\
\text { ingth, head, arm, and thigh } \\
\text { s } \\
\text { overweight, obesity } \\
\text { r failure to thrive, stunting, and } \\
\text { ren }\end{array}$ \\
\hline
\end{tabular}

Key Definitions

Complementary feeding is defined as the process that starts when human milk or infant formula is complemented by other foods and beverages. The complementary feeding period typically continues to 24 months as the young child transitions fully to family foods.

Complementary foods and beverages (CFB) are foods and beverages (liquids, semisolids, and solids) other than human milk or infant formula provided to an infant or young child to provide nutrients and energy.

\begin{tabular}{|ll}
\hline Key Confounders & Other Confounders \\
\hline - Education & - Smoking \\
- Socioeconomic status & - Maternal diet \\
- Sex & - Maternal weight \\
- Maternal age & - Maternal parity \\
- Race and/or ethnicity & - Mildcare arrangement \\
- Milkfeeding practices & - Marital status/head of household \\
(breast milk, infant & - Medical support/access \\
- Birmula, or both) & Participation in a supplemental \\
- Gestational age & - Atopy risk status \\
& - Maternal employment/returning to \\
& - Infant sleep patterns \\
& - Baseline growth status \\
\hline
\end{tabular}




\section{SEARCH PLAN AND RESULTS}

\section{Inclusion and exclusion criteria}

This table provides the inclusion and exclusion criteria for the systematic review question on timing of introduction of complementary foods and beverages and growth, size, and body composition. The inclusion and exclusion criteria are a set of characteristics to determine which studies will be included or excluded in the systematic review.

Table 2. Inclusion and exclusion criteria

\begin{tabular}{|c|c|c|}
\hline Category & Inclusion Criteria & Exclusion Criteria \\
\hline Study design & $\begin{array}{l}\text { - Randomized controlled trials } \\
\text { - Non-randomized controlled trials } \\
\text { - Prospective cohort studies } \\
\text { - Retrospective cohort studies } \\
\text { - Case-control studies } \\
\text { - Pre/post studies with a control }\end{array}$ & $\begin{array}{l}\text { - Cross-sectional studies } \\
\text { - Uncontrolled studies } \\
\text { - Pre/post studies without a control } \\
\text { - Narrative reviews } \\
\text { - Systematic reviews } \\
\text { - Meta-analyses }\end{array}$ \\
\hline $\begin{array}{l}\text { Independent variable } \\
\text { (intervention or } \\
\text { exposure) }\end{array}$ & $\begin{array}{l}\text { Timing of introduction of CFB. CFB are foods } \\
\text { and beverages other than human milk or infant } \\
\text { formula (liquids, semisolids, and solids) provided } \\
\text { to an infant or young child to provide nutrients } \\
\text { and energy. }\end{array}$ & $\begin{array}{l}\text { Isolated consumption of human milk, infant } \\
\text { formulas (e.g., milk-based, soy, partially- } \\
\text { hydrolyzed, extensive-hydrolyzed, amino acid- } \\
\text { based), fluid cow's milk before } 12 \text { months of } \\
\text { age, or vitamin and mineral supplements (e.g., } \\
\text { iron drops) }\end{array}$ \\
\hline Comparator & Different timing of introduction of CFB & $N / A$ \\
\hline $\begin{array}{l}\text { Dependent variables } \\
\text { (outcomes) }\end{array}$ & $\begin{array}{l}\text { - Weight and length/height } \\
\text { - BMI, BMI percentile for age and z-score } \\
\text { - Waist circumference } \\
\text { - Weight change } \\
\text { - Weight status change } \\
\text { - Weight-for-age, length/stature-for-age, weight- } \\
\text { for-length, head, arm, and thigh circumference } \\
\text { for age } \\
\text { - Change across more than one time point of } \\
\text { weight-for age, length-for-age, weight-for- }\end{array}$ & $\mathrm{N} / \mathrm{A}$ \\
\hline
\end{tabular}




\begin{tabular}{|c|c|c|}
\hline & $\begin{array}{l}\text { length, head, arm, and thigh circumference for } \\
\text { age } \\
\text { - \% fat mass, \% fat free mass } \\
\text { - Skinfold thickness } \\
\text { - Incidence of healthy weight, overweight, } \\
\text { obesity } \\
\text { - Incidence of underweight or failure to thrive, } \\
\text { stunting, and wasting in infants and children }\end{array}$ & \\
\hline Date range & • January 1980 - July 2016 & \\
\hline Language & - Studies published in English & $\begin{array}{l}\text { - Studies published in languages other than } \\
\text { English }\end{array}$ \\
\hline Publication status & - Studies published in peer-reviewed journals & $\begin{array}{l}\text { - Grey literature, including unpublished data, } \\
\text { manuscripts, reports, abstracts, conference } \\
\text { proceedings }\end{array}$ \\
\hline Country ${ }^{1}$ & $\begin{array}{l}\text { - Studies conducted in Very High or High } \\
\text { Human Development Countries }\end{array}$ & $\begin{array}{l}\text { - Studies conducted in Medium or Low Human } \\
\text { Development Countries }\end{array}$ \\
\hline Study participants & $\begin{array}{l}\text { - Human subjects } \\
\text { - Males } \\
\text { - Females }\end{array}$ & $\begin{array}{l}\text { - Hospitalized patients, not including birth and } \\
\text { immediate post-partum hospitalization of healthy } \\
\text { babies }\end{array}$ \\
\hline $\begin{array}{l}\text { Age of study } \\
\text { participants }\end{array}$ & $\begin{array}{l}\text { - Age at intervention or exposure: } \\
\text { o Infants (0-12 months) } \\
\text { oToddlers (12-24 months) } \\
\text { - Age at outcome: } \\
\text { o Infants (0-12 months) } \\
\text { oToddlers (12-24 months) } \\
\text { o Child (2-5 years) } \\
\text { o Child (6-12 years) } \\
\text { o Adolescents (13-18 years) } \\
\text { o Adults (19 and older) } \\
\text { o Older adults ( } 65 \text { to } 79 \text { years) } \\
\text { o Older adults ( } 80+\text { years) }\end{array}$ & $\begin{array}{l}\text { - Age at intervention or exposure: } \\
\text { ○Child (2-5 years) } \\
\circ \text { Child (6-12 years) } \\
\text { oAdolescents (13-18 years) } \\
\circ \text { Adults (19 and older) } \\
\text { ○Older adults (65 to } 79 \text { years) } \\
\text { ○Older adults ( } 80+\text { years) }\end{array}$ \\
\hline Health status of study & - Studies done in generally healthy populations & - Studies that exclusively enroll subjects with a \\
\hline
\end{tabular}


- Studies done in populations where infants were full term ( $\geq 37$ weeks gestational age)

- Studies done in populations with elevated chronic disease risk, or that enroll some participants with a disease or with the health outcome of interest disease or with the health outcome of interest

- Studies done in hospitalized or malnourished subjects

- Studies of exclusively pre-term babies (gestational age $<37$ weeks) or babies that are small for gestational age $(<2500 \mathrm{~g})$

- Studies of subjects with infectious diseases (e.g. HIV/AIDS) (or with mothers diagnosed with an infectious disease)

${ }^{1}$ The ratings of country development (e.g., high, medium, low, very low) were based on the Human Development Report 2014 (82). When a country was not included in the Human Development Report 2014 ranking, country classification from the World Bank was used instead (84). Medium Development countries were originally included, but due to concerns about generalizability to the U.S. of study participants (i.e., baseline health status) and complementary foods and beverages typically consumed, a decision was made to exclude "Medium" countries in October 2017 


\section{Search terms and electronic databases used}

\section{PubMed:}

Date(s) Searched: 7/19/2016

Search Terms:

Final:

Complementary OR supplementary OR wean* OR transition* OR introduc* OR "Infant Nutritional Physiological Phenomena"[Mesh:noexp] OR weaning[mesh] OR ((bottle*) NOT (milk OR formula))

AND (feeding ${ }^{*}$ OR food OR beverage ${ }^{*}[$ tiab] OR beverages[mh] OR eating OR diet[tiab] OR $\operatorname{diet}[\mathrm{mh}]$ OR meal*[tiab] OR meals[mh] OR "Food and Beverages"[Mesh] OR diets[tiab] OR cereal*[tiab] OR "Edible Grain"[Mesh] OR bread*[tiab] OR whole grain* OR juice*[tiab] OR milk[tiab] OR "Milk"[Mesh] OR dairy[tiab] OR "Dairy Products"[Mesh] OR meat[tiab] OR cheese[tiab] OR yogurt[tiab] OR yoghurt*[tiab] OR fruit"[tiab] OR "Fruit"[Mesh] OR vegetable*[tiab] OR "Vegetables"[Mesh] OR egg*[tiab] OR "Eggs"[Mesh] OR nut[tiab] OR nuts[tiab] OR peas[tiab] OR beans[tiab] OR legume*[tiab] OR snack*[tiab] OR bread[mh] OR honey[mh] OR vegetable*[tiab] OR "Vegetables"[Mesh] OR egg*[tiab] OR "Eggs"[Mesh:noexp] OR "egg white"[mh] OR "egg yolk"[mh] OR snack*[tiab] OR candy[mh] OR "Fast Foods"[Mesh] OR meat[mh] OR molasses[mh] OR nuts[mh] OR "Raw Foods"[Mesh] OR seeds[mh])

OR "infant food"[mesh]

OR infant feed*

OR (breast feeding[mh] OR breastfeeding[tiab] OR breast feeding*[tiab] OR breast-feeding*[tiab] OR breastfed[tiab] OR breast-fed[tiab] OR breast-feed OR breast-feeds)

OR (Bottle feeding[mh] OR bottle feeding*[tiab]s OR bottle feeding OR bottle-feeding*[tiab] OR bottlefeedings OR bottle-fed[tiab] OR "bottle fed"[tiab])

NOT (editorial[ptyp] OR comment[ptyp] OR news[ptyp] OR letter[ptyp] OR review[ptyp] OR systematic[sb])

OR ((Solid food $\left.{ }^{*}\right)$ OR solids)); AND

Growth[mh:noexp] OR "Child Development"[Mesh] OR "Growth Charts"[Mesh] OR "growth and development" [Subheading] OR "growth and development"[tiab] OR "Growth and Development"[Mesh:noexp] OR "Growth"[tiab] OR development*[tiab] OR "Child Development"[Mesh] OR child develop*[tiab] OR tooth[mh] OR tooth[tiab] OR teeth[tiab] OR movement[mh] OR "Overnutrition"[Mesh] OR under-nutrition[tiab] OR undernutrition[tiab] OR "developmental delay"[tiab] OR "Motor Skills"[Mesh] OR "Nonverbal Communication"[Mesh] 
Standing[tiab] OR sitting[tiab] OR walking[tiab] OR crawling[tiab] OR "Motor Skills"[Mesh] OR Ages and Stages Questionnaire* OR ASQ[tiab]

OR Cognitive[tiab] OR cognition[mh] OR cognition OR learning OR "Learning Disorders"[Mesh] OR "Intellectual Disability"[Mesh] OR intelligence[tiab] OR intelligence[mh] OR "Achievement"[Mesh] OR "Aptitude"[Mesh] OR "Executive Function"[Mesh] OR memory OR inhibitory control*[tiab] OR "problem solving"[tiab] OR"Social-emotional development"[tiab] OR "Neurological development"[tiab] OR "mental development"[tiab] OR "Motor development"[tiab] OR anxiety[tiab] OR anxiety[mh:noexp] OR "Anxiety, Separation"[Mesh] OR depression[tiab] OR depression[mh] OR "Depression, Postpartum"[Mesh] OR "Depressive Disorder"[Mesh] OR

"Visual Acuity"[Mesh] OR "Auditory Perception"[Mesh] OR "Psychological Tests"[Mesh]

OR

("Bone Density"[Mesh] OR "bone density"[tiab] OR "Bone Development"[Mesh] OR "Bone Development"[tiab] OR "Fractures, Bone"[Mesh] OR "Bone Diseases"[Mesh] OR osteoporosis[tiab] OR (bone[tiab] AND fracture*[tiab]) OR "Rickets"[Mesh] OR ricket*[tiab] OR bone mineral*[tiab] OR "bone mass"[tiab] OR bone health"[tiab] OR "Bone Demineralization, Pathologic"[Mesh] OR bone demineral*[tiab])

OR

("body size"[tiab] OR body size[mh] OR obesity[tiab] OR obese[tiab] OR overweight[mh] OR obesity[mh] OR overweight [tiab] OR adipos*[tiab] OR adiposity[mh] OR "body composition"[mh] OR body fat distribution[mh] OR "body fat"[tiab] OR "body weight"[tiab] OR body weight[mh] OR birth weight*[tiab] OR weight gain[mh] OR weight loss[mh] OR "body-weight"[tiab] OR "weight gain"[tiab] OR weight-gain[tiab] OR weight loss[tiab] OR weight-loss[tiab] OR "Body Weights and Measures"[mh] OR weight[ti] OR "Anthropometry"[Mesh:noexp] OR body mass index[mh] OR "body mass index"[tiab] OR BMI[tiab] OR "weight status"[tiab] OR "adipose tissue"[mh] OR "healthy weight"[tiab] OR waist circumference[mh] OR "body mass"[ti] OR "fat mass"[tiab] OR body weight changes[mh] OR "waist circumference"[tiab] OR ideal body weight[mh] OR waist-hip ratio[mh] OR Waist Hip*[tiab] OR body height*[tiab] OR Crown-Rump Length*[tiab] OR head circumference ${ }^{*}\left[\right.$ tiab] OR arm circumference* ${ }^{*}$ tiab] OR thigh circumference* OR limb circumference* OR fat free mass*[tiab] OR skinfold[tiab] OR skin fold*[tiab])

AND

infant* OR baby OR babies OR toddler* OR newborn*[tiab] OR "Child, Preschool"[Mesh] OR preschool*[tiab] OR pre-school*[tiab] OR "early childhood"[tiab] OR "early years"[tiab] OR prek[tiab] OR pre-primary[tiab] OR under five*[ti] OR young child*[ti] OR "head start"[tiab] OR prekindergarten[tiab] OR pre-kindergarten[tiab] OR weanling*

OR limit to child, preschool

for child 0-18 all develop outcomes

for all; body wgt/comp/bone

NOT

nutritional status[mh] OR nutritional status*[tiab] OR Nutrition Status ${ }^{\star}[\operatorname{tiab}]$ OR Iron[mh] OR 
iron[tiab] OR "Anemia"[Mesh] OR "Anemia"[tiab] OR iron deficien*[tiab] OR ferritin*[tiab] OR ferrous[tiab] OR "Transferrin"[Mesh] OR "Transferrin"[tiab] OR zinc OR "Vitamin D"[Mesh] OR "Vitamin D"[tiab] OR "Vitamin D Deficiency"[Mesh] OR "Vitamin B 12"[Mesh] OR "Vitamin B 12"[tiab] OR "Vitamin B12"[tiab] OR "Vitamin B 12 Deficiency"[Mesh] OR Cobamide*[tiab] OR Cobalamin*[tiab] OR Cyanocobalamin[tiab] OR Folate[tiab] OR "Folic Acid"[Mesh] OR folacin[tiab] OR vitamin b9*[tiab] OR Fatty acid*[tiab] OR "Fatty Acids"[Mesh:noexp] OR fatty acid*[tiab] OR "Fatty Acids, Unsaturated"[Mesh:noexp] OR Arachidonic acid*[tiab] OR linolenic acid*[tiab] OR linoleic acid*[tiab] OR Docosahexaenoic Acid*[tiab] OR Eicosapentaenoic Acid*[tiab] OR gamma-Linolenic Acid"[tiab] OR "Arachidonic Acids"[Mesh] OR "Fatty Acids, Essential"[Mesh] OR "Fatty Acids, Omega-3"[Mesh] OR "Fatty Acids, Omega-6"[Mesh] OR alphaLinolenic Acid*[tiab] OR "Fatty Acids, Essential"[Mesh] OR "Linolenic Acids"[Mesh] OR "Trans Fatty Acids"[Mesh] OR "Fatty Acids, Monounsaturated"[Mesh]

for nonmedline[sb]: NOT animals by: NOT (sheep[ti] OR lamb[ti] OR lambs[ti] OR calving[ti] OR calves[ti] OR mice[ti] OR mouse[ti] OR pigs[ti] OR cows[ti] OR piglets[ti] OR cow[ti] OR piglet[ti] OR monkey[ti] OR rats[ti] OR rat[ti] OR animal $\left.{ }^{*}[\mathrm{ti}]\right)$

\section{Embase:}

Date(s) Searched: 8/1/16

Search Terms:

(Complementary OR supplementa* OR wean* OR transition* OR introduc* OR family) NEAR/3 (feed $^{*}$ OR food ${ }^{*}$ OR beverage* OR eating OR diet)

OR

(Complementary OR transition* OR introduct* OR wean*) AND (food/exp OR 'baby food'/exp OR 'cereal'/exp OR 'dairy product'/exp OR 'egg'/exp OR 'fruit'/exp OR 'meat'/exp OR 'sea food'/exp OR 'milk'/exp OR fish/exp OR 'poultry'/exp OR 'beverage'/exp OR 'vegetable'/exp OR nut/exp OR pea/exp OR meal/exp)

OR

(Complementary OR supplementa* OR wean* OR transition* OR introduc*) NEAR/5 ('whole grain' OR 'whole grains' OR dairy OR egg OR eggs OR meat OR poultry OR seafood OR fruit* OR milk OR fish* OR poultry OR beverage* OR vegetables* OR pea OR peas OR nut OR nuts OR cereal OR bread* OR yog*urt* OR cheese* OR juice* OR rice OR soup OR legume* OR snack $^{\star}$ OR meal ${ }^{\star}$ ) (for Embase)

OR 'baby food'/de OR (solid NEAR/2 food*):ab,ti

AND

(infant*:ti,ab OR infant/exp) OR (baby OR babies OR toddler* OR newborn* OR nurser ${ }^{\star}$ ):ti,ab OR 'newborn'/exp OR 'newborn care'/exp OR preschool*:ti,ab OR pre-school:ti,ab OR 'preschool child'/exp OR 'infancy'/exp OR "early childhood":ti,ab OR "early years" OR pre-k:ti,ab OR 'nursery'/exp OR 'nursery school'/exp OR prekindergarten:ti,ab OR pre-kindergarten:ti,ab OR weanling* 
AND ([in process]/lim OR [article]/lim OR [article in press]/lim) AND ([embase]/lim NOT [medline]/lim)

AND

Limit to humans:

AND

'executive function'/exp OR 'executive function':ti,ab OR 'learning'/exp OR 'intelligence'/exp OR 'mental development'/exp OR 'mental development':ti,ab OR intelligence:ti,ab OR cogniti*:ti,ab OR 'cognition'/exp OR 'cognition assessment'/exp OR aptitude:ti,ab OR 'memory'/exp OR memory:ti,ab OR 'anxiety'/exp OR 'anxiety':ti,ab OR 'depression'/exp OR depressi*:ti,ab OR 'visual acuity'/exp OR visual:ti OR 'hearing'/exp OR hearing:ti,ab OR auditory:ti,ab OR 'postnatal development'/exp OR 'postnatal development':ti,ab OR 'overnutrition'/exp OR 'overnutrition':ti,ab OR undernutrition:ti,ab OR “developmental delay":ti,ab OR 'nonverbal communication'/exp OR

('metabolic bone disease'/exp OR osteoporosis:ti,ab OR (bone NEAR/2 (disease* OR fracture* OR injur* OR health* OR density OR mineralize* OR demineraliz*)):ti,ab OR ricket*:ti,ab OR 'bone injury'/exp OR 'bone density'/exp)

AND

'body size'/de OR 'body size':ti,ab OR 'obesity'/exp OR overweight:ab,ti OR 'macrosomia'/exp OR obese:ab,ti OR obesity:ab,ti OR 'weight gain':ab,ti OR adiposity:ab,ti OR adipose:ab,ti OR 'body weight'/exp OR 'body weight':ti,ab OR 'weight gain'/de OR 'body composition'/exp OR 'body composition':ti,ab OR 'body fat':ab,ti OR 'anthropometry'/de OR 'body mass'/de OR bmi:ab,ti OR 'body mass':ab,ti OR weight:ab,ti OR (waist NEXT/1 hip NEXT/1 ratio*) OR 'body fat'/de OR 'adipose tissue'/exp OR skinfold OR 'skin fold':ti,ab OR 'fat mass':ti,ab OR 'fat mass'/exp OR 'anthropometric parameters'/exp OR circumference OR length OR height

OR

'body growth'/exp 'body growth':ti,ab OR 'growth rate and growth regulation'/exp OR 'postnatal growth'/exp OR 'human development'/exp OR 'Bayley Scales of Infant Development'/exp OR 'cognition assessment'/exp OR 'mental function assessment'/de

\section{Cochrane:}

Date(s) Searched: 8/9/16

Search Terms:

(feed ${ }^{*}$ OR food* OR beverage* OR diet* OR 'whole grain' OR 'whole grains' OR dairy OR egg OR meat OR poultry OR seafood OR fruit* OR milk OR fish* OR poultry OR vegetables* OR pea OR beans OR legume ${ }^{\star}$ OR nut OR cereal OR beverage* OR bread* OR seafood OR yog ${ }^{\star}$ urt $^{\star}$ OR cheese OR juice OR snack OR yogurt OR yoghurt OR nut OR nuts OR honey OR meal OR meals) NEAR/3 (Complementary OR supplementa* OR wean* OR transition* OR introduct* OR family) 
OR

[mh ^"Infant Nutritional Physiological Phenomena"] OR [mh weaning] OR ((bottle*) NOT (milk OR formula))

AND ([mh beverages] OR [mh eating] OR [mh diet] OR [mh meals] OR [mh "Food and Beverages"] OR [mh "Edible Grain"] OR [mh "Milk"] OR dairy[:ti,ab OR [mh "Dairy Products"] OR [mh "Fruit"] OR [mh "Vegetables"] OR [mh "Eggs"] OR [mh bread] OR [mh honey] OR [mh "Vegetables"] OR [mh ^"Eggs"] OR [mh "egg white"] OR [mh "egg yolk"] OR [mh candy] OR [mh "Fast Foods"] OR [mh meat] OR [mh molasses] OR [mh nuts] OR [mh "Raw Foods"] OR [mh seeds])

OR

((Infant* OR baby* OR babies) NEAR/2 food*):ti,ab OR [mh "infant food"]

AND

[mh ^Growth] OR [mh "Child Development"] OR [mh "Growth Charts"] OR "growth and development" OR [mh ^"Growth and Development"] OR [mh "Child Development"] OR (child NEAR/1 develop*):ti,ab OR [mh tooth] OR tooth:ti,ab OR teeth:ti,ab OR [mh movement] OR [mh "Overnutrition"] OR "under-nutrition:ti,ab OR undernutrition:ti,ab OR [mh "Motor Skills"] OR [mh "Nonverbal Communication"]

OR

'body growth':ti,ab OR 'growth rate and growth regulation' OR 'postnatal growth':ti,ab OR 'human development':ti,ab OR 'Bayley Scales of Infant Development'

OR Standing:ti,ab OR sitting:ti,ab OR walking:ti,ab OR crawling:ti,ab OR "Ages and Stages Questionnaire" OR ASQ:ti,ab

OR [mh cognition] OR [mh learning] OR [mh "Learning Disorders"] OR [mh "Intellectual

Disability"] OR intelligence:ti,ab OR [mh intelligence] OR [mh "Achievement"] OR [mh "Aptitude"] OR [mh "Executive Function"] OR (inhibitory NEAR/1 control*):ti,ab OR "problem solving":ti,ab OR "Social-emotional development":ti, ab OR "Neurological development":ti,ab OR "mental development":ti,ab OR "Motor development"ti,ab OR [mh ^anxiety] OR [mh "Anxiety, Separation"] OR [mh depression] OR [mh "Depression, Postpartum"] OR [mh "Depressive Disorder"] OR [mh "Visual Acuity"] OR [mh "Auditory Perception"] OR [mh "Psychological Tests"]

OR Stunt*:ti,ab OR wasting:ti,ab

OR

cogniti*:ti,ab OR aptitude:ti,ab OR memory:ti,ab OR [mh memory] OR 'anxiety':ti,ab OR depressi*:ti,ab OR visual:ti,ab OR vision:ti,ab OR hearing:ti,ab OR auditory:ti,ab OR 'postnatal development':ti, ab OR 'overnutrition':ti,ab OR “developmental delay”:ti,ab OR 'nonverbal communication'

OR 
[mh "Bone Density"] OR [mh "Bone Development"] OR [mh "Fractures, Bone"] OR [mh "Bone Diseases"] OR [mh "Rickets"] OR [mh "Bone Demineralization, Pathologic"] OR osteoporosis:ti,ab OR (bone NEAR/2 (disease* OR fracture* OR injur* OR health* OR density OR mineral* OR demineral $^{*}$ OR develop* OR mass)):ti,ab OR ricket $^{\star}:$ ti,ab

\section{OR}

'body size':ti,ab OR overweight:ab,ti OR 'macrosomia':ti,ab OR obese:ab,ti OR obesity:ab,ti OR adipos*:ab,ti OR 'body weight':ti,ab OR 'weight gain':ti,ab OR 'body composition':ti,ab OR 'body fat':ab,ti OR 'anthropometr' ${ }^{*}: t i$, ab OR bmi:ab,ti OR 'body mass':ab,ti OR (waist NEXT/1 hip NEXT/1 ratio*) OR 'body fat':ti,ab OR 'adipose tissue':ti,ab OR skinfold:ti,ab OR 'skin fold':ti,ab OR 'fat mass':ti,ab OR circumference:ti,ab OR length:ti,ab OR height:ti,ab

([mh "body size"] OR [mh overweight] OR [mh obesity] OR [mh adiposity] OR [mh "body composition"] OR [mh "body fat distribution"] OR [mh "body weight"] OR [mh "weight gain"] OR [mh "weight loss"] OR "weight gain":ti,ab OR "weight loss":ti,ab OR "weight-loss":ti,ab OR [mh "Body Weights and Measures"] OR weight:ti OR [mh ^"Anthropometry"] OR [mh "body mass index"] OR "weight status":ti, ab OR [mh "adipose tissue"] OR "healthy weight":ti,ab OR [mh "waist circumference"] OR [mh "body weight changes"] OR [mh "ideal body weight"] OR [mh "waist-hip ratio"] OR "Waist Hip":ti,ab OR “waist-hip":ti,ab OR “Crown-Rump”:ti,ab OR "fat free mass":ti,ab)

NOT (pubmed OR embase)

\section{CINAHL}

Date(s) Searched: 8/22/2016

Search Terms:

(MH "Food and Beverages+") OR (MH "Food") OR (MH "Diet") OR (MH "Eating") OR (MH "Eating Behavior") OR (MH "Taste") OR (MH "Taste Buds") OR (MH "Cereals") OR (MH "Dairy Products") OR (MH "Yogurt") OR (MH "Cheese") OR (MH "Milk") OR (MH "Eggs") OR (MH "Fruit") OR (MH "Fruit Juices") OR (MH "Meat") OR (MH "Seafood") OR (MH "Fish") OR (MH "Poultry") OR (MH "Vegetables") OR (MH "Nuts") OR (MH "Legumes") OR (MH "Bread") AND (Complementary OR supplementa* OR wean* OR transition* OR introduc*)

OR

('whole grain' OR 'whole grains' OR dairy OR egg OR eggs OR meat OR poultry OR seafood OR fruit* OR milk OR fish* OR poultry OR vegetables* OR pea OR peas OR nut OR nuts OR cereal OR beverage* OR bread* OR seafood OR yog ${ }^{*}$ urt $^{*}$ OR cheese* OR juice*) N5 (Complementary OR supplementa* OR wean* OR transition* OR introduc* OR family)

OR (Infant* OR baby OR babies) N2 food ${ }^{*}$

NOT

(MH "Nutritional Status") OR "nutritional status" OR (MH "Nutritional Requirements") OR (MH "Vitamin D") OR (MH "Vitamin D Deficiency") OR (MH "Vitamin B12 Deficiency") OR (MH "Anemia") OR "anemia" OR (MH "Anemia, Iron Deficiency") OR (MH "Iron") OR (MH "Zinc") OR (MH "Vitamin B12") OR (MH "Vitamin B12 Deficiency") OR (MH "Folic Acid") OR (MH "Niacin") 
OR (MH "Folic Acid Deficiency") OR "folate" OR "folacin" OR cyanocobalamin* OR cobalamin* OR cobamamide* OR (MH "Fatty Acids") OR "fatty acids" OR (MH "Fatty Acids, Omega-6") OR (MH "Fatty Acids, Omega-3") OR (MH "Fatty Acids, Unsaturated") OR (MH "Trans Fatty Acids") OR (MH "Fatty Acids, Monounsaturated") OR (MH "Fatty Acids, Saturated") OR (MH "Fatty Acids, Essential") OR (MH "Arachidonic Acids") OR (MH "Docosahexaenoic Acids") OR (MH "Linolenic Acids") OR (MH "Linoleic Acids")

\section{AND}

osteoporosis OR (bone n2 (disease* OR fracture* OR injur* OR health* OR density OR mineralize* OR demineraliz*)) OR ricket* OR (MH "Osteoporosis") OR (MH "Bone Density") OR (MH "Bone Diseases+") OR (MH "Bone Diseases, Developmental+") OR (MH "Rickets+")

OR

(MH "Executive Function") OR (MH "Learning+") OR (MH "Intelligence+") OR "intelligence" OR (MH "Intelligence Tests") OR (MH "Cognition+") OR "cognition" OR "mental development" OR (MH "Aptitude") OR "aptitude" OR (MH "Aptitude Tests+") OR (MH "Memory+") OR "memory" OR (MH "Anxiety+") OR "anxiety" OR (MH "Depression+") OR "depression" OR (MH "Visual Acuity") OR (MH "Visual Perception+") OR (MH "Hearing+") OR "auditory" OR "overnutrition" OR "undernutrition" OR (MH "Nonverbal Communication+") OR "postnatal development" OR OR "developmental delay"

OR

(MH "Anthropometry+") OR (MH "Body Weights and Measures+") OR (MH "Body Weight+") OR (MH "Bone Development+") OR (MH "Growth+") OR (MH "Human Development+") OR "bayley scales" OR "mental function" OR (MH "Body Size") OR (MH "Obesity+") OR "overweight" OR "macrosomia" OR (MH "Weight Gain+") OR (MH "Waist-Hip Ratio") OR (MH "Body Composition+") OR (MH "Adipose Tissue+") OR (MH "Abdominal Fat") OR (MH "Fat Free Mass") OR (MH "Body Mass Index") OR (MH "Skinfold Thickness") OR (MH "Head Circumference") OR (MH "Arm Circumference") OR (MH "Waist Circumference") OR (MH "Growth and Development (Omaha)") OR (MH "Body Height") OR (MH "Crown-Rump Length") OR (MH "Leg Length Inequality") OR (MH "Mean Length of Utterance") 
Figure 2: Flow chart of literature search and screening results

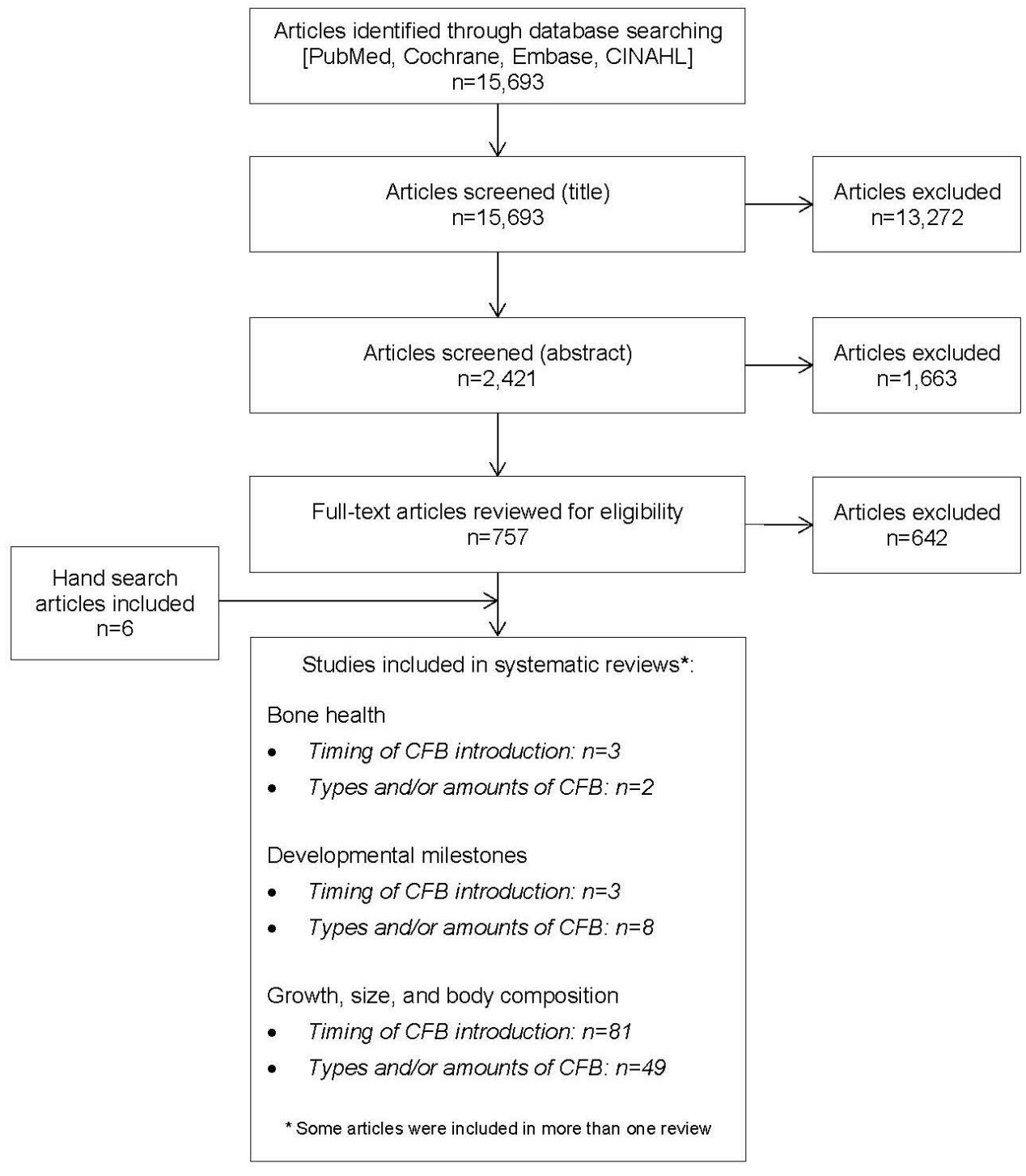

This flow chart illustrates the literature search results for articles examining the relationship between complementary feeding and growth, size, and/or body composition. The results of an electronic database search were screened independently by two NESR analysts by reviewing titles, abstracts, and full text articles to determine which articles met the criteria for inclusion. A manual search was done to ascertain articles not identified through the electronic database search. The systematic review on timing of introduction of CFB included 81 articles, and the systematic review on types and amounts of CFB consumed included 49 articles. The literature search was conducted for multiple systematic reviews that addressed complementary feeding and various health outcomes. 


\section{Excluded articles}

The table below lists the excluded articles with at least one reason for exclusion, but may not reflect all possible reasons.

\section{Table 3. Excluded articles}

\begin{tabular}{|c|c|c|}
\hline & Citation & Rationale $^{1}$ \\
\hline 1 & Complementary feeding in the WHO Multicentre Growth Reference Study. Acta Paediatr Suppl.2006;450:27-37. & DV \\
\hline 2 & $\begin{array}{l}\text { Weaning and the weaning diet. Report of the Working Group on the Weaning Diet of the Committee on Medical Aspects of Food } \\
\text { Policy. Rep Health Soc Subj (Lond).1994;45:1-113. }\end{array}$ & Design \\
\hline 3 & $\begin{array}{l}\text { Aarts, C.,Kylberg, E.,Hofvander, Y.,Gebre-Medhin, M. Growth under privileged conditions of healthy Swedish infants exclusively } \\
\text { breastfed from birth to 4-6 months: a longitudinal prospective study based on daily records of feeding. Acta Paediatr.2003;92:145- } \\
51 .\end{array}$ & IV \\
\hline 4 & $\begin{array}{l}\text { Abarin, T.,Yan Wu, Y.,Warrington, N.,Lye, S.,Pennell, C.,Briollais, L. The impact of breastfeeding on FTO-related BMI growth } \\
\text { trajectories: an application to the Raine pregnancy cohort study. Int J Epidemiol.2012;41:1650-60. }\end{array}$ & IV \\
\hline 5 & $\begin{array}{l}\text { Abou Samra, H.,Stevens, D.,Binkley, T.,Specker, B. Determinants of bone mass and size in 7-year-old former term, late-preterm, } \\
\text { and preterm boys. Osteoporos Int.2009;20:1903-10. }\end{array}$ & Design, IV \\
\hline 6 & $\begin{array}{l}\text { Aboud, F. E.,Akhter, S. A cluster-randomized evaluation of a responsive stimulation and feeding intervention in bangladesh. } \\
\text { Pediatrics.2011;127:e1191-7. }\end{array}$ & IV \\
\hline 7 & $\begin{array}{l}\text { Aboud, F. E.,Shafique, S.,Akhter, S. A responsive feeding intervention increases children's self-feeding and maternal } \\
\text { responsiveness but not weight gain. J Nutr.2009;139:1738-43. }\end{array}$ & IV \\
\hline 8 & $\begin{array}{l}\text { Adu-Afarwuah, S.,Lartey, A.,Brown, K. H.,Zlotkin, S.,Briend, A.,Dewey, K. G. Randomized comparison of } 3 \text { types of micronutrient } \\
\text { supplements for home fortification of complementary foods in Ghana: effects on growth and motor development. Am J Clin } \\
\text { Nutr.2007;86:412-20. }\end{array}$ & IV \\
\hline 9 & $\begin{array}{l}\text { Agarwal, K. N.,Agarwal, D. K.,Gupta, A.,Bansal, A. K. Relationship of exclusive breast feeding for } 6 \text { mo to linear growth up to } 18 \text { mo } \\
\text { of age. Indian J Pediatr.2013;80:11-5. }\end{array}$ & Country \\
\hline 10 & $\begin{array}{l}\text { Aggarwal, A.,Arora, S.,Patwari, A. K. Breastfeeding among urban women of low-socioeconomic status: factors influencing } \\
\text { introduction of supplemental feeds before four months of age. Indian Pediatr.1998;35:269-73. }\end{array}$ & $\begin{array}{l}\text { Design, } \\
\text { IV,DV }\end{array}$ \\
\hline 11 & $\begin{array}{l}\text { Agostoni, C.,Fiocchi, A.,Riva, E.,Terracciano, L.,Sarratud, T.,Martelli, A.,Lodi, F.,D'Auria, E.,Zuccotti, G.,Giovannini, M. Growth of } \\
\text { infants with IgE-mediated cow's milk allergy fed different formulas in the complementary feeding period. Pediatr Allergy } \\
\text { Immunol.2007;18:599-606. }\end{array}$ & IV \\
\hline 12 & $\begin{array}{l}\text { Agostoni, C.,Grandi, F.,Gianni, M. L.,Silano, M.,Torcoletti, M.,Giovannini, M.,Riva, E. Growth patterns of breast fed and formula fed } \\
\text { infants in the first } 12 \text { months of life: an Italian study. Arch Dis Child.1999;81:395-9. }\end{array}$ & IV \\
\hline 13 & $\begin{array}{l}\text { Agostoni, C.,Grandi, F.,Scaglioni, S.,Gianni, M. L.,Torcoletti, M.,Radaelli, G.,Fiocchi, A.,Riva, E. Growth pattern of breastfed and } \\
\text { nonbreastfed infants with atopic dermatitis in the first year of life. Pediatrics.2000;106:E73. }\end{array}$ & IV \\
\hline 14 & $\begin{array}{l}\text { Agostoni,C.,Marangoni,F.,Lammardo,A. M.,Giovannini,M.,Riva,E.,Galli,C. Breastfeeding duration, milk fat composition and } \\
\text { developmental indices at } 1 \text { year of life among breastfed infants. Prostaglandins Leukot Essent Fatty Acids.2001;64:105-9. }\end{array}$ & IV \\
\hline
\end{tabular}




\begin{tabular}{|c|c|c|}
\hline 15 & $\begin{array}{l}\text { Agostoni,C.,Zuccotti,G. V.,Radaelli,G.,Besana,R.,Podesta,A.,Sterpa,A.,Rottoli,A.,Riva,E.,Giovannini,M. Docosahexaenoic acid } \\
\text { supplementation and time at achievement of gross motor milestones in healthy infants: a randomized, prospective, double-blind, } \\
\text { placebo-controlled trial. Am J Clin Nutr.2009;89:64-70. }\end{array}$ & IV \\
\hline 16 & $\begin{array}{l}\text { Allen, L.,Shrimpton, R. The International Research on Infant Supplementation study: implications for programs and further research. } \\
\text { J Nutr.2005;135:666s-669s. }\end{array}$ & Design \\
\hline 17 & $\begin{array}{l}\text { Alm,B.,Aberg,N.,Erdes,L.,Mollborg,P.,Pettersson,R.,Norvenius,S. G.,Goksor,E.,Wennergren,G. Early introduction of fish decreases } \\
\text { the risk of eczema in infants. Arch Dis Child.2009;94:11-5. }\end{array}$ & DV \\
\hline 18 & $\begin{array}{l}\text { Almqvist,C.,Garden,F.,Xuan,W.,Mihrshahi,S.,Leeder,S. R.,Oddy,W.,Webb,K.,Marks,G. B. Omega-3 and omega-6 fatty acid } \\
\text { exposure from early life does not affect atopy and asthma at age } 5 \text { years. J Allergy Clin Immunol.2007;119:1438-44. }\end{array}$ & IV, DV \\
\hline 19 & $\begin{array}{l}\text { Alvarez-Uria, G.,Midde, M.,Pakam, R.,Bachu, L.,Naik, P. K. Effect of Formula Feeding and Breastfeeding on Child Growth, Infant } \\
\text { Mortality, and HIV Transmission in Children Born to HIV-Infected Pregnant Women Who Received Triple Antiretroviral Therapy in a } \\
\text { Resource-Limited Setting: Data from an HIV Cohort Study in India. ISRN Pediatr.2012;2012:763591. }\end{array}$ & $\begin{array}{l}\text { Health } \\
\text { statu }\end{array}$ \\
\hline 20 & $\begin{array}{l}\text { Andersen, L. B.,Molgaard, C.,Michaelsen, K. F.,Carlsen, E. M.,Bro, R.,Pipper, C. B. Indicators of dietary patterns in Danish infants at } \\
9 \text { months of age. Food Nutr Res.2015;59:27665. }\end{array}$ & Design \\
\hline 21 & $\begin{array}{l}\text { Andersen,A. D.,Michaelsen,K. F.,Hellgren,L. I.,Trolle,E.,Lauritzen,L. A randomized controlled intervention with fish oil versus } \\
\text { sunflower oil from } 9 \text { to } 18 \text { months of age: exploring changes in growth and skinfold thicknesses. Pediatr Res.2011;70:368-74. }\end{array}$ & IV \\
\hline 22 & $\begin{array}{l}\text { Andersen,L. B.,Pipper,C. B.,Trolle,E.,Bro,R.,Larnkjaer,A.,Carlsen,E. M.,Molgaard,C.,Michaelsen,K. F. Maternal obesity and offspring } \\
\text { dietary patterns at } 9 \text { months of age. Eur J Clin Nutr.2015;69:668-75. }\end{array}$ & DV \\
\hline 23 & $\begin{array}{l}\text { Anderson, G. H.,Morson-Pasut, L. A.,Bryan, H.,Cleghorn, G.,Tanaka, P.,Yeung, D.,Zimmerman, B. Age of introduction of cow's milk } \\
\text { to infants. J Pediatr Gastroenterol Nutr.1985;4:692-8. }\end{array}$ & Design \\
\hline 24 & $\begin{array}{l}\text { Anderson, V. P.,Cornwall, J.,Jack, S.,Gibson, R. S. Intakes from non-breastmilk foods for stunted toddlers living in poor urban } \\
\text { villages of Phnom Penh, Cambodia, are inadequate. Matern Child Nutr.2008;4:146-59. }\end{array}$ & $\begin{array}{l}\text { Design, } \\
\text { Health } \\
\text { status }\end{array}$ \\
\hline 25 & $\begin{array}{l}\text { Andres, A.,Casey, P. H.,Cleves, M. A.,Badger, T. M. Body fat and bone mineral content of infants fed breast milk, cow's milk } \\
\text { formula, or soy formula during the first year of life. J Pediatr.2013;163:49-54. }\end{array}$ & IV \\
\hline 26 & $\begin{array}{l}\text { Andres, A.,Cleves, M. A.,Bellando, J. B.,Pivik, R. T.,Casey, P. H.,Badger, T. M. Developmental status of 1-year-old infants fed } \\
\text { breast milk, cow's milk formula, or soy formula. Pediatrics.2012;129:1134-40. }\end{array}$ & IV \\
\hline 27 & $\begin{array}{l}\text { Andrissi, L.,Mottini, G.,Sebastiani, V.,Boldrini, L.,Giuliani, A. Dietary habits and growth: an urban/rural comparison in the Andean } \\
\text { region of Apurimac, Peru. Ann Ist Super Sanita.2013;49:340-6. }\end{array}$ & IV \\
\hline 28 & Anfield,L. Nutrition in the first year. Midwife Health Visit Community Nurse.1985;21:161-4. & Design \\
\hline 29 & $\begin{array}{l}\text { Anzman-Frasca, S.,Liu, S., Gates, K. M.,Paul, I. M.,Rovine, M. J.,Birch, L. L. Infants' Transitions out of a Fussing/Crying State Are } \\
\text { Modifiable and Are Related to Weight Status. Infancy.2013;18:662-686. }\end{array}$ & IV \\
\hline 30 & Armstrong, J.,Reilly, J. J. Breastfeeding and lowering the risk of childhood obesity. Lancet.2002;359:2003-4. & IV \\
\hline 31 & $\begin{array}{l}\text { Arsenault,J. E.,Havel,P. J.,Lopez de Romana,D.,Penny,M. E.,Van Loan,M. D.,Brown,K. H. Longitudinal measures of circulating } \\
\text { leptin and ghrelin concentrations are associated with the growth of young Peruvian children but are not affected by zinc } \\
\text { supplementation. Am J Clin Nutr.2007;86:1111-9. }\end{array}$ & $\begin{array}{l}\text { Health } \\
\text { status }\end{array}$ \\
\hline
\end{tabular}




\begin{tabular}{|c|c|c|}
\hline 32 & Arvas, A.,Elgormus, Y.,Gur, E.,Alikasifoglu, M.,Celebi, A. Iron status in breast-fed full-term infants. Turk J Pediatr.2000;42:22-6. & IV \\
\hline 33 & $\begin{array}{l}\text { Asha Bai, P. V.,Leela, M.,Subramaniam, V. R. Adequacy of breast milk for optimal growth of infants. Trop Geogr Med.1980;32:158- } \\
62 .\end{array}$ & IV \\
\hline 34 & $\begin{array}{l}\text { Assuncao, M. L.,Ferreira, H. S.,Coutinho, S. B.,Santos, L. M.,Horta, B. L. Protective effect of breastfeeding against overweight can } \\
\text { be detected as early as the second year of life: a study of children from one of the most socially-deprived areas of Brazil. J Health } \\
\text { Popul Nutr.2015;33:85-91. }\end{array}$ & $\begin{array}{l}\text { Design, } \\
\text { Health } \\
\text { status, IV }\end{array}$ \\
\hline 35 & $\begin{array}{l}\text { Atladottir, H.,Thorsdottir, I. Energy intake and growth of infants in Iceland-a population with high frequency of breast-feeding and } \\
\text { high birth weight. Eur J Clin Nutr.2000;54:695-701. }\end{array}$ & IV \\
\hline 36 & Auestad,N. Infant nutrition--brain development--disease in later life. An introduction. Dev Neurosci.2000;22:472-3. & Design \\
\hline 37 & $\begin{array}{l}\text { Augusto,R. A.,Souza,J. M. Effectiveness of a supplementary feeding program in child weight gain. Rev Saude Publica.2010;44:793- } \\
801 .\end{array}$ & Design, IV \\
\hline 38 & $\begin{array}{l}\text { Axelsson,I. E.,Jakobsson,I.,Raiha,N. C. Formula with reduced protein content: effects on growth and protein metabolism during } \\
\text { weaning. Pediatr Res.1988;24:297-301. }\end{array}$ & IV \\
\hline 39 & $\begin{array}{l}\text { Azad, M. B.,Konya, T.,Maughan, H.,Guttman, D. S.,Field, C. J.,Chari, R. S.,Sears, M. R.,Becker, A. B.,Scott, J. A., Kozyrskyj, A. L. } \\
\text { Gut microbiota of healthy Canadian infants: profiles by mode of delivery and infant diet at } 4 \text { months. Cmaj.2013;185:385-94. }\end{array}$ & DV \\
\hline 40 & Badger, T. Effects of soy infant formula on growth and development in the first year of life. Food Nutr Bull.2013;34:252-3. & Design, IV \\
\hline 41 & $\begin{array}{l}\text { Bahamondes L,Bahamondes MV,Modesto W,Tilley IB,Magalhaes A,Pinto e Silva JL,Amaral E, Jr. Mishell DR. Effect of hormonal } \\
\text { contraceptives during breastfeeding on infant's milk ingestion and growth. Fertil Steril.2013;100:445-50. }\end{array}$ & IV \\
\hline 42 & $\begin{array}{l}\text { Bai, K. I.,Sastry, V. N.,Reddy, C. C. A comparative study of feeding pattern of infants in rural and urban areas. Indian J } \\
\text { Pediatr.1981;48:277-80. }\end{array}$ & Design, IV \\
\hline 43 & $\begin{array}{l}\text { Balaban, G.,Motta, M. E.,Silva, G. A. Early weaning and other potential risk factors for overweight among preschool children. Clinics } \\
\text { (Sao Paulo).2010;65:181-7. }\end{array}$ & IV, Age \\
\hline 44 & $\begin{array}{l}\text { Balogun,T. A.,Lombard,M. J.,McLachlan,M. The nutrient intake of children aged 12-36 months living in two communities in the } \\
\text { Breede Valley, Western Cape province, South Africa. South African Family Practice.2015;57:1-7 7p. }\end{array}$ & Design \\
\hline 45 & $\begin{array}{l}\text { Baranowski, T.,Bryan, G. T.,Harrison, J. A.,Rassin, D. K.,Greaves, K. A.,Baranowski, J. H. Height, infant-feeding practices and } \\
\text { cardiovascular functioning among } 3 \text { or } 4 \text { year old children in three ethnic groups. J Clin Epidemiol.1992;45:513-8. }\end{array}$ & DV \\
\hline 46 & $\begin{array}{l}\text { Bartok, C. J.,Schaefer, E. W.,Beiler, J. S.,Paul, I. M. Role of body mass index and gestational weight gain in breastfeeding } \\
\text { outcomes. Breastfeed Med.2012;7:448-56. }\end{array}$ & IV, DV \\
\hline 47 & Beal, V. A. Nutrition and growth-patterns of young children. ASDC J Dent Child.1983;50:139-41. & Design \\
\hline 48 & $\begin{array}{l}\text { Begum, H. A.,Mascie-Taylor, C.,Nahar, S. The impact of food supplementation on infant weight gain in rural Bangladesh; an } \\
\text { assessment of the Bangladesh Integrated Nutritional Program (BINP). Public Health Nutr.2007;10:49-54. }\end{array}$ & IV \\
\hline 49 & $\begin{array}{l}\text { Beinner,M. A.,Velasquez-Melendez,G.,Pessoa,M. C.,Greiner,T. Iron-fortified rice is as efficacious as supplemental iron drops in } \\
\text { infants and young children. J Nutr.2010;140:49-53. }\end{array}$ & IV, DV \\
\hline 50 & Ben,X. M.,Zhou,X. Y.,Zhao,W. H.,Yu,W. L.,Pan,W.,Zhang,W. L.,Wu,S. M.,Van Beusekom,C. M.,Schaafsma,A. Growth and & IV \\
\hline
\end{tabular}




\begin{tabular}{|c|c|c|}
\hline & $\begin{array}{l}\text { development of term infants fed with milk with long-chain polyunsaturated fatty acid supplementation. Chin Med J } \\
\text { (Engl).2004;117:1268-70. }\end{array}$ & \\
\hline 51 & $\begin{array}{l}\text { Bennett,W. E.,Jr.,Hendrix,K. S.,Thompson-Fleming,R. T.,Downs,S. M.,Carroll,A. E. Early cow's milk introduction is associated with } \\
\text { failed personal-social milestones after } 1 \text { year of age. Eur J Pediatr.2014;173:887-92. }\end{array}$ & IV \\
\hline 52 & $\begin{array}{l}\text { Bergmann, K. E.,Bergmann, R. L.,Von Kries, R.,Bohm, O.,Richter, R.,Dudenhausen, J. W.,Wahn, U. Early determinants of } \\
\text { childhood overweight and adiposity in a birth cohort study: role of breast-feeding. Int J Obes Relat Metab Disord.2003;27:162-72. }\end{array}$ & IV \\
\hline 53 & $\begin{array}{l}\text { Bernal, M. J.,Periago, M. J.,Martinez, R.,Ortuno, I.,Sanchez-Solis, M.,Ros, G.,Romero, F.,Abellan, P. Effects of infant cereals with } \\
\text { different carbohydrate profiles on colonic function--randomised and double-blind clinical trial in infants aged between } 6 \text { and } 12 \\
\text { months--pilot study. Eur J Pediatr.2013;172:1535-42. }\end{array}$ & IV \\
\hline 54 & $\begin{array}{l}\text { Berni Canani R,Nocerino R,Terrin G,Frediani T,Lucarelli S,Cosenza L,Passariello A,Leone L,Granata V,Di Costanzo M,Pezzella } \\
\text { V,Troncone R. Formula selection for management of children with cow's milk allergy influences the rate of acquisition of tolerance: a } \\
\text { prospective multicenter study. J Pediatr.2013;163:771-7.e1. }\end{array}$ & IV, DV \\
\hline 55 & $\begin{array}{l}\text { Betoko, A.,Charles, M. A.,Hankard, R.,Forhan, A.,Bonet, M.,Regnault, N.,Botton, J.,Saurel-Cubizolles, M. J.,de Lauzon-Guillain, B. } \\
\text { Determinants of infant formula use and relation with growth in the first } 4 \text { months. Matern Child Nutr.2014;10:267-79. }\end{array}$ & IV \\
\hline 56 & $\begin{array}{l}\text { Betoko, A.,Charles, M. A.,Hankard, R.,Forhan, A.,Bonet, M.,Saurel-Cubizolles, M. J.,Heude, B.,de Lauzon-Guillain, B. Infant feeding } \\
\text { patterns over the first year of life: influence of family characteristics. Eur J Clin Nutr.2013;67:631-7. }\end{array}$ & DV \\
\hline 57 & $\begin{array}{l}\text { Bhandari, N.,Bahl, R.,Nayyar, B.,Khokhar, P.,Rohde, J. E.,Bhan, M. K. Food supplementation with encouragement to feed it to } \\
\text { infants from } 4 \text { to } 12 \text { months of age has a small impact on weight gain. J Nutr.2001;131:1946-51. }\end{array}$ & Country \\
\hline 58 & $\begin{array}{l}\text { Bhandari, N.,Mazumder, S.,Bahl, R.,Martines, J.,Black, R. E.,Bhan, M. K. An educational intervention to promote appropriate } \\
\text { complementary feeding practices and physical growth in infants and young children in rural Haryana, India. J Nutr.2004;134:2342-8. }\end{array}$ & IV \\
\hline 59 & $\begin{array}{l}\text { Bhatia, B. D.,Banerjee, D.,Agarwal, D. K.,Agarwal, K. N. Exterogestate growth: relationship with maternal body size and dietary } \\
\text { intakes. Indian J Pediatr.1983;50:241-6. }\end{array}$ & $\begin{array}{l}\text { Health } \\
\text { status, IV }\end{array}$ \\
\hline 60 & $\begin{array}{l}\text { Bindon, J. R.,Cabrera, C. Infant feeding patterns and growth of infants in American Samoa during the first year of life. Hum } \\
\text { Biol.1988;60:81-92. }\end{array}$ & $\begin{array}{l}\text { Design, IV, } \\
\text { DV }\end{array}$ \\
\hline 61 & $\begin{array}{l}\text { Bindon, J. R. The body build and composition of Samoan children: relationships to infant feeding patterns and infant weight-for- } \\
\text { length status. Am J Phys Anthropol.1984;63:379-88. }\end{array}$ & IV \\
\hline 62 & Bindon, J. R. The influence of infant feeding patterns on growth of children in American Samoa. Med Anthropol.1985;9:183-95. & Country \\
\hline 63 & $\begin{array}{l}\text { Birch,E. E.,Garfield,S.,Castaneda,Y.,Hughbanks-Wheaton,D.,Uauy,R.,Hoffman,D. Visual acuity and cognitive outcomes at } 4 \text { years } \\
\text { of age in a double-blind, randomized trial of long-chain polyunsaturated fatty acid-supplemented infant formula. Early Hum } \\
\text { Dev.2007;83:279-84. }\end{array}$ & IV \\
\hline 64 & $\begin{array}{l}\text { Bisimwa, G.,Owino, V. O.,Bahwere, P.,Dramaix, M.,Donnen, P.,Dibari, F.,Collins, S. Randomized controlled trial of the effectiveness } \\
\text { of a soybean-maize-sorghum-based ready-to-use complementary food paste on infant growth in South Kivu, Democratic Republic of } \\
\text { Congo. Am J Clin Nutr.2012;95:1157-64. }\end{array}$ & IV \\
\hline 65 & $\begin{array}{l}\text { Bjorke-Monsen, A. L. Is exclusive breastfeeding ensuring an optimal micronutrient status and psychomotor development in infants?. } \\
\text { Clin Biochem.2014;47:714. }\end{array}$ & Design \\
\hline 66 & Block,S. L. Delayed introduction of solid foods to infants: not so fast!. Pediatr Ann.2013;42:143-7. & Design \\
\hline
\end{tabular}




\begin{tabular}{|c|c|c|}
\hline 67 & $\begin{array}{l}\text { Bogen, D. L.,Hanusa, B. H.,Whitaker, R. C. The effect of breast-feeding with and without formula use on the risk of obesity at } 4 \\
\text { years of age. Obes Res.2004;12:1527-35. }\end{array}$ & IV \\
\hline 68 & $\begin{array}{l}\text { Bonuck, K.,Avraham, S. B.,Lo, Y.,Kahn, R.,Hyden, C. Bottle-weaning intervention and toddler overweight. J Pediatr.2014;164:306- } \\
\text { 12.e1-2. }\end{array}$ & IV \\
\hline 69 & $\begin{array}{l}\text { Borschel, M. W.,Baggs, G. E.,Barrett-Reis, B. Growth of healthy term infants fed ready-to-feed and powdered forms of an } \\
\text { extensively hydrolyzed casein-based infant formula: a randomized, blinded, controlled trial. Clin Pediatr (Phila).2014;53:585-92. }\end{array}$ & IV \\
\hline 70 & $\begin{array}{l}\text { Boulton J. Nutrition in childhood and its relationships to early somatic growth, body fat, blood pressure, and physical fitness. Acta } \\
\text { Paediatr Scand Suppl.1981;284:1-85. }\end{array}$ & Design,DV \\
\hline 71 & $\begin{array}{l}\text { Brakohiapa, L. A.,Yartey, J.,Bille, A.,Harrison, E.,Quansah, E.,Armar, M. A.,Kishi, K.,Yamamoto, S. Does prolonged breastfeeding } \\
\text { adversely affect a child's nutritional status?. Lancet.1988;2:416-8. }\end{array}$ & IV, DV \\
\hline 72 & $\begin{array}{l}\text { Brazionis, L.,Golley, R. K.,Mittinty, M. N.,Smithers, L. G.,Emmett, P.,Northstone, K.,Lynch, J. W. Diet spanning infancy and } \\
\text { toddlerhood is associated with child blood pressure at age } 7.5 \text { y. Am J Clin Nutr.2013;97:1375-86. }\end{array}$ & DV \\
\hline 73 & $\begin{array}{l}\text { Breij, L. M.,Mulder, M. T.,van Vark-van der Zee, L. C.,Hokken-Koelega, A. C. Appetite-regulating hormones in early life and } \\
\text { relationships with type of feeding and body composition in healthy term infants. Eur J Nutr.2016. }\end{array}$ & IV \\
\hline 74 & $\begin{array}{l}\text { Brekke, H. K.,van Odijk, J.,Ludvigsson, J. Predictors and dietary consequences of frequent intake of high-sugar, low-nutrient foods } \\
\text { in 1-year-old children participating in the ABIS study. Br J Nutr.2007;97:176-81. }\end{array}$ & DV \\
\hline 75 & $\begin{array}{l}\text { Brew,B. K.,Toelle,B. G.,Webb,K. L.,Almqvist,C.,Marks,G. B. Omega-3 supplementation during the first } 5 \text { years of life and later } \\
\text { academic performance: a randomised controlled trial. Eur J Clin Nutr.2015;69:419-24. }\end{array}$ & IV \\
\hline 76 & $\begin{array}{l}\text { Briend,A.,Bari,A. Breastfeeding improves survival, but not nutritional status, of 12-35 months old children in rural Bangladesh. Eur J } \\
\text { Clin Nutr.1989;43:603-8. }\end{array}$ & $\begin{array}{l}\text { Health } \\
\text { status, IV }\end{array}$ \\
\hline 77 & $\begin{array}{l}\text { Briend,A.,Darmon,N. Determining limiting nutrients by linear programming: A new approach to predict insufficient intakes from } \\
\text { complementary foods. Pediatrics.2000;106:1288-9. }\end{array}$ & Design \\
\hline 78 & $\begin{array}{l}\text { Brito,A.,Olivares,M.,Pizarro,T.,Rodriguez,L.,Hertrampf,E. Chilean complementary feeding program reduces anemia and improves } \\
\text { iron status in children aged } 11 \text { to } 18 \text { months. Food Nutr Bull.2013;34:378-85. }\end{array}$ & Design \\
\hline 79 & Brown A,Lee MD. Early influences on child satiety-responsiveness: the role of weaning style. Pediatr Obes.2015;10:57-66. & IV \\
\hline 80 & $\begin{array}{l}\text { Brown, A.,Lee, M. Breastfeeding during the first year promotes satiety responsiveness in children aged 18-24 months. Pediatr } \\
\text { Obes.2012;7:382-90. }\end{array}$ & IV \\
\hline 81 & $\begin{array}{l}\text { Brown,K. H.,Lopez de Romana,D.,Arsenault,J. E.,Peerson,J. M.,Penny,M. E. Comparison of the effects of zinc delivered in a } \\
\text { fortified food or a liquid supplement on the growth, morbidity, and plasma zinc concentrations of young Peruvian children. Am J Clin } \\
\text { Nutr.2007;85:538-47. }\end{array}$ & IV \\
\hline 82 & $\begin{array}{l}\text { Brown,L. V.,Zeitlin,M. F.,Peterson,K. E.,Chowdhury,A. M.,Rogers,B. L.,Weld,L. H.,Gershoff,S. N. Evaluation of the impact of } \\
\text { weaning food messages on infant feeding practices and child growth in rural Bangladesh. Am J Clin Nutr.1992;56:994-1003. }\end{array}$ & IV \\
\hline 83 & $\begin{array}{l}\text { Brulotte, J.,Bukutu, C.,Vohra, S. Complementary, holistic, and integrative medicine: fish oils and neurodevelopmental disorders. } \\
\text { Pediatr Rev.2009;30:e29-33. }\end{array}$ & Design \\
\hline 84 & $\begin{array}{l}\text { Bulk-Bunschoten, A. M.,van Bodegom, S., Reerink, J. D.,de Jong, P. C., de Groot, C. J. Weight and weight gain at } 4 \text { months (The } \\
\text { Netherlands 1998): influences of nutritional practices, socio-economic and ethnic factors. Paediatr Perinat Epidemiol.2002;16:361- } \\
\text { 9. }\end{array}$ & IV \\
\hline
\end{tabular}




\begin{tabular}{|c|c|c|}
\hline 85 & $\begin{array}{l}\text { Burnham, L.,Matlak, S.,Makrigiorgos, G.,Braun, N.,Knapp, B. P.,Merewood, A. Breastfeeding and coffee consumption in children } \\
\text { younger than } 2 \text { years in Boston, Massachusetts, USA. J Hum Lact.2015;31:267-72. }\end{array}$ & DV \\
\hline 86 & $\begin{array}{l}\text { Caleyachetty A,Krishnaveni GV,Veena SR,Hill J,Karat SC,Fall CH,Wills AK. Breastfeeding duration, age of starting solids and high } \\
\text { BMI risk and adiposity in Indian children. Matern Child Nutr.2013;9:199-216. }\end{array}$ & $\begin{array}{l}\text { Design,Co } \\
\text { untry }\end{array}$ \\
\hline 87 & Calvo,E. B.,Galindo,A. C.,Aspres,N. B. Iron status in exclusively breast-fed infants. Pediatrics.1992;90:375-9. & IV, DV \\
\hline 88 & $\begin{array}{l}\text { Calvo,E.,Hertrampf,E.,Pablo,S.,Amar,M.,Stekel,A. Haemoglobin-fortified cereal: an alternative weaning food with high iron } \\
\text { bioavailability. European journal of clinical nutrition.1989;43:237-43. }\end{array}$ & $\begin{array}{l}\text { Design, } \\
\text { DV }\end{array}$ \\
\hline 89 & $\begin{array}{l}\text { Cameron, S. L.,Heath, A. L.,Gray, A. R.,Churcher, B.,Davies, R. S.,Newlands, A., Galland, B. C.,Sayers, R. M., Lawrence, J. } \\
\text { A.,Taylor, B. J.,Taylor, R. W. Lactation Consultant Support from Late Pregnancy with an Educational Intervention at } 4 \text { Months of Age } \\
\text { Delays the Introduction of Complementary Foods in a Randomized Controlled Trial. J Nutr.2015;145:1481-90. }\end{array}$ & DV \\
\hline 90 & $\begin{array}{l}\text { Capozzi,L.,Russo,R.,Bertocco,F.,Ferrara,D.,Ferrara,M. Diet and iron deficiency in the first year of life: a retrospective study. } \\
\text { Hematology.2010;15:410-3. }\end{array}$ & DV \\
\hline 91 & $\begin{array}{l}\text { Capozzi,L.,Russo,R.,Bertocco,F.,Ferrara,D.,Ferrara,M. Effect on haematological and anthropometric parameters of iron } \\
\text { supplementation in the first } 2 \text { years of life. Risks and benefits. Hematology.2011;16:261-4. }\end{array}$ & IV \\
\hline 92 & $\begin{array}{l}\text { Carruth,B. R.,Nevling,W.,Skinner,J. D. Developmental and food profiles of infants born to adolescent and adult mothers. J Adolesc } \\
\text { Health.1997;20:434-41. }\end{array}$ & $\begin{array}{l}\text { IV, DV, } \\
\text { Age }\end{array}$ \\
\hline 93 & $\begin{array}{l}\text { Casiday, R. E.,Wright, C. M.,Panter-Brick, C.,Parkinson, K. N. Do early infant feeding patterns relate to breast-feeding continuation } \\
\text { and weight gain? Data from a longitudinal cohort study. Eur J Clin Nutr.2004;58:1290-6. }\end{array}$ & IV \\
\hline 94 & $\begin{array}{l}\text { Castillo-Duran,C.,Perales,C. G.,Hertrampf,E. D.,Marin,V. B.,Rivera,F. A.,Icaza,G. Effect of zinc supplementation on development } \\
\text { and growth of Chilean infants. J Pediatr.2001;138:229-35. }\end{array}$ & IV \\
\hline 95 & $\begin{array}{l}\text { Chaimay, B.,Ruagdaraganon, N.,Thinkhamrop, B., Thinkhamrop, J. Association between infant feeding practices and first meaningful } \\
\text { words at first year of life: a prospective cohort study of Thai children. Asia Pac J Public Health.2015;27:Np1071-84. }\end{array}$ & IV \\
\hline 96 & $\begin{array}{l}\text { Chan,G. M.,Roberts,C. C.,Folland,D.,Jackson,R. Growth and bone mineralization of normal breast-fed infants and the effects of } \\
\text { lactation on maternal bone mineral status. Am J Clin Nutr.1982;36:438-43. }\end{array}$ & IV \\
\hline 97 & $\begin{array}{l}\text { Chang, S. Y.,He, W.,Chen, C. M. Complementary feeding and growth of infant and young child in China. Biomed Environ } \\
\text { Sci.2008;21:264-8. }\end{array}$ & Design \\
\hline 98 & Chatterjee, M. K. Feeding pattern practised by the mothers attending an under five clinic. Indian J Public Health.1987;31:268-9. & Design \\
\hline 99 & Chavez A,Martinez C,Schlaepfer L. Health effects of supplementary feeding programs. Prog Clin Biol Res.1981;67:129-39. & Design \\
\hline 100 & Chawla, P.,Puri, R.,Pershad, D. Impact of pre-school supplementary feeding on mental abilities. Indian Pediatr.1983;20:513-6. & Age \\
\hline 101 & Chawla, P.,Puri, R. Impact of pre-school supplementary feeding programme on anthropometry. Indian Pediatr.1983;20:363-7. & Age \\
\hline 102 & $\begin{array}{l}\text { Cheng, T. S.,Loy, S. L.,Cheung, Y. B.,Chan, J. K.,Pang, W. W.,Godfrey, K. M.,Gluckman, P. D.,Kwek, K.,Saw, S. M.,Chong, Y. } \\
\text { S.,Lee, Y. S.,Lek, N.,Yap, F. Sexually dimorphic response to feeding mode in the growth of infants. Am J Clin Nutr.2016;103:398- } \\
405 .\end{array}$ & IV \\
\hline
\end{tabular}




\begin{tabular}{|c|c|c|}
\hline 103 & $\begin{array}{l}\text { Chirmulay, D.,Nisal, R. Nutritional status of tribal underfive children in Ahmadnagar District, Maharashtra in relation to } \\
\text { weaning/feeding practices. Indian Pediatr.1993;30:215-22. }\end{array}$ & Design \\
\hline 104 & $\begin{array}{l}\text { Chitra, P. Development of banana-based weaning food mixes for infants and its nutritional quality evaluation. Rev Environ } \\
\text { Health.2015;30:125-30. }\end{array}$ & IV \\
\hline 105 & $\begin{array}{l}\text { Chivers, P.,Hands, B.,Parker, H.,Bulsara, M.,Beilin, L. J.,Kendall, G. E.,Oddy, W. H. Body mass index, adiposity rebound and early } \\
\text { feeding in a longitudinal cohort (Raine Study). Int J Obes (Lond).2010;34:1169-76. }\end{array}$ & IV \\
\hline 106 & $\begin{array}{l}\text { Cho, H. N.,Hong, S.,Lee, S. H.,Yum, H. Y. Nutritional status according to sensitized food allergens in children with atopic dermatitis. } \\
\text { Allergy, Asthma and Immunology Research.2010;3:53-57. }\end{array}$ & $\begin{array}{l}\text { Design, } \\
\text { IV, DV }\end{array}$ \\
\hline 107 & $\begin{array}{l}\text { Chorell,E.,Karlsson Videhult,F.,Hernell,O.,Antti,H.,West,C. E. Impact of probiotic feeding during weaning on the serum lipid profile } \\
\text { and plasma metabolome in infants. Br J Nutr.2013;110:116-26. }\end{array}$ & IV \\
\hline 108 & $\begin{array}{l}\text { Choudhury, N.,Bromage, S.,Alam, M. A.,Ahmed, A. M.,Islam, M. M.,Hossain, M. I.,Mahfuz, M.,Mondal, D.,Haque, M. R.,Ahmed, T. } \\
\text { Intervention study shows suboptimal growth among children receiving a food supplement for five months in a slum in Bangladesh. } \\
\text { Acta Paediatr.2016. }\end{array}$ & $\begin{array}{l}\text { Health } \\
\text { status, IV }\end{array}$ \\
\hline 109 & Christoffel, K. A pediatric perspective on vegetarian nutrition. Clin Pediatr (Phila).1981;20:632-43. & Design \\
\hline 110 & Christopher, G. C. First food: the essential role of breastfeeding. Breastfeed Med.2009;4 Suppl 1:S9-s10. & Design \\
\hline 111 & $\begin{array}{l}\text { Chuang, C. H.,Yang, S. H.,Chang, P. J.,Chen, P. C.,Chen, Y. C. Dietary supplement intake by 6-month-old Taiwanese infants. J } \\
\text { Pediatr Gastroenterol Nutr.2012;54:71-6. }\end{array}$ & DV \\
\hline 112 & $\begin{array}{l}\text { Closa-Monasterolo, R.,Gispert-Llaurado, M.,Luque, V.,Ferre, N.,Rubio-Torrents, C.,Zaragoza-Jordana, M., Escribano, J. Safety and } \\
\text { efficacy of inulin and oligofructose supplementation in infant formula: results from a randomized clinical trial. Clin Nutr.2013;32:918- } \\
27 .\end{array}$ & IV \\
\hline 113 & $\begin{array}{l}\text { Cloutier, M. M.,Wiley, J.,Wang, Z.,Grant, A.,Gorin, A. A. The Early Childhood Obesity Prevention Program (ECHO): an ecologically- } \\
\text { based intervention delivered by home visitors for newborns and their mothers. BMC Public Health.2015;15:584. }\end{array}$ & Design \\
\hline 114 & Cockburn,F. Neonatal brain and dietary lipids. Arch Dis Child Fetal Neonatal Ed.1994;70:F1-2. & Design \\
\hline 115 & $\begin{array}{l}\text { Cohen RJ,Brown KH,Canahuati J,Rivera LL,Dewey KG. Effects of age of introduction of complementary foods on infant breast milk } \\
\text { intake, total energy intake, and growth: a randomised intervention study in Honduras. Lancet.1994;344:288-93. }\end{array}$ & Country \\
\hline 116 & $\begin{array}{l}\text { Cohen, R. J.,Brown, K. H.,Canahuati, J.,Rivera, L. L.,Dewey, K. G. Determinants of growth from birth to } 12 \text { months among breast- } \\
\text { fed Honduran infants in relation to age of introduction of complementary foods. Pediatrics.1995;96:504-10. }\end{array}$ & IV \\
\hline 117 & Coleman,B. L. Early introduction of non-formula cow's milk to southern Ontario infants. Can J Public Health.2006;97:187-90. & IV, DV \\
\hline 118 & $\begin{array}{l}\text { Colombo,J.,Carlson,S. E.,Cheatham,C. L.,Shaddy,D. J.,Kerling,E. H.,Thodosoff,J. M.,Gustafson,K. M.,Brez,C. Long-term effects of } \\
\text { LCPUFA supplementation on childhood cognitive outcomes. Am J Clin Nutr.2013;98:403-12. }\end{array}$ & IV \\
\hline 119 & $\begin{array}{l}\text { Coppi, S., lacoponi, F.,Fommei, C.,Strambi, M. Growth trend during the first six months of life in male infants with different type of } \\
\text { feeding. Minerva Pediatr.2013;65:51-9. }\end{array}$ & IV \\
\hline 120 & $\begin{array}{l}\text { Costom, B. H.,Shore, D. Effect of a comprehensive nutritional program on the growth and ponderosity of infants. Clin Pediatr } \\
\text { (Phila).1983;22:105-11. }\end{array}$ & Design \\
\hline
\end{tabular}




\begin{tabular}{|c|c|c|}
\hline 121 & $\begin{array}{l}\text { Courage, M. L.,McCloy, U. R.,Herzberg, G. R.,Andrews, W. L.,Simmons, B. S.,McDonald, A. C.,Mercer, C. N.,Friel, J. K. Visual } \\
\text { acuity development and fatty acid composition of erythrocytes in full-term infants fed breast milk, commercial formula, or evaporated } \\
\text { milk. J Dev Behav Pediatr.1998;19:9-17. }\end{array}$ & IV \\
\hline 122 & Cusack, R. Dietary management of obese children and adolescents. Pediatr Ann.1984;13:455, 458-9, 462-4. & Design \\
\hline 123 & $\begin{array}{l}\text { Dagan, R.,Sofer, S.,Klish, W. J.,Hundt, G.,Saltz-Rennert, H.,Moses, S. W. Infant feeding practices among Bedouins in transition } \\
\text { from seminomadic to settlement conditions in the Negev area of Israel. Isr J Med Sci.1984;20:1029-34. }\end{array}$ & $\begin{array}{l}\text { Design, } \\
\text { DV }\end{array}$ \\
\hline 124 & $\begin{array}{l}\text { Dagnelie,P. C.,van Staveren,W. A.,Hautvast,J. G. Stunting and nutrient deficiencies in children on alternative diets. Acta Paediatr } \\
\text { Scand Suppl.1991;374:111-8. }\end{array}$ & DV \\
\hline 125 & $\begin{array}{l}\text { Dagnelie,P. C.,van Staveren,W. A.,Vergote,F. J.,Dingjan,P. G.,van den Berg,H.,Hautvast,J. G. Increased risk of vitamin B-12 and } \\
\text { iron deficiency in infants on macrobiotic diets. Am J Clin Nutr.1989;50:818-24. }\end{array}$ & $\begin{array}{l}\text { Design, } \\
\text { DV }\end{array}$ \\
\hline 126 & $\begin{array}{l}\text { Dagnelie,P. C.,van Staveren,W. A.,Verschuren,S. A.,Hautvast,J. G. Nutritional status of infants aged } 4 \text { to } 18 \text { months on macrobiotic } \\
\text { diets and matched omnivorous control infants: a population-based mixed-longitudinal study. I. Weaning pattern, energy and nutrient } \\
\text { intake. Eur J Clin Nutr.1989;43:311-23. }\end{array}$ & $\begin{array}{l}\text { Design, } \\
\text { DV }\end{array}$ \\
\hline 127 & $\begin{array}{l}\text { Daniels, L. A.,Mallan, K. M.,Battistutta, D.,Nicholson, J. M.,Meedeniya, J. E.,Bayer, J. K.,Magarey, A. Child eating behavior } \\
\text { outcomes of an early feeding intervention to reduce risk indicators for child obesity: the NOURISH RCT. Obesity (Silver } \\
\text { Spring).2014;22:E104-11. }\end{array}$ & DV \\
\hline 128 & $\begin{array}{l}\text { Daniels, L. A.,Mallan, K. M.,Battistutta, D.,Nicholson, J. M.,Perry, R.,Magarey, A. Evaluation of an intervention to promote protective } \\
\text { infant feeding practices to prevent childhood obesity: outcomes of the NOURISH RCT at } 14 \text { months of age and } 6 \text { months post the } \\
\text { first of two intervention modules. Int J Obes (Lond).2012;36:1292-8. }\end{array}$ & IV, DV \\
\hline 129 & $\begin{array}{l}\text { Daniels, L. A.,Mallan, K. M.,Nicholson, J. M.,Battistutta, D.,Magarey, A. Outcomes of an early feeding practices intervention to } \\
\text { prevent childhood obesity. Pediatrics.2013;132:e109-18. }\end{array}$ & IV \\
\hline 130 & $\begin{array}{l}\text { Daniels, L. A.,Mallan, K. M.,Nicholson, J. M.,Thorpe, K.,Nambiar, S.,Mauch, C. E.,Magarey, A. An Early Feeding Practices } \\
\text { Intervention for Obesity Prevention. Pediatrics.2015;136:e40-9. }\end{array}$ & IV \\
\hline 131 & Daniels, M. C.,Adair, L. S. Breast-feeding influences cognitive development in Filipino children. J Nutr.2005;135:2589-95. & IV \\
\hline 132 & $\begin{array}{l}\text { Davidsson,L.,Sarker,S. A.,Jamil,K. A.,Sultana,S.,Hurrell,R. Regular consumption of a complementary food fortified with ascorbic } \\
\text { acid and ferrous fumarate or ferric pyrophosphate is as useful as ferrous sulfate in maintaining hemoglobin concentrations }>105 \mathrm{~g} / \mathrm{L} \\
\text { in young Bangladeshi children. Am J Clin Nutr.2009;89:1815-20. }\end{array}$ & IV, DV \\
\hline 133 & $\begin{array}{l}\text { de Hoog ML,van Eijsden M,Stronks K,Gemke RJ,Vrijkotte TG. The role of infant feeding practices in the explanation for ethnic } \\
\text { differences in infant growth: the Amsterdam Born Children and their Development study. Br J Nutr.2011;106:1592-601. }\end{array}$ & Design \\
\hline 134 & $\begin{array}{l}\text { de Kanashiro, H. C.,Brown, K. H.,Lopez de Romana, G.,Lopez, T.,Black, R. E. Consumption of food and nutrients by infants in } \\
\text { Huascar (Lima), Peru. Am J Clin Nutr.1990;52:995-1004. }\end{array}$ & DV \\
\hline 135 & $\begin{array}{l}\text { de Villiers, A.,Senekal, M. Determinants of growth failure in 12-24-month-old children in a high-density urban slum community in } \\
\text { East London, South Africa. Eur J Clin Nutr.2002;56:1231-41. }\end{array}$ & Country \\
\hline 136 & $\begin{array}{l}\text { de Zegher, F.,Sebastiani, G.,Diaz, M.,Sanchez-Infantes, D.,Lopez-Bermejo, A., Ibanez, L. Body composition and circulating high- } \\
\text { molecular-weight adiponectin and IGF-I in infants born small for gestational age: breast- versus formula-feeding. } \\
\text { Diabetes.2012;61:1969-73. }\end{array}$ & $\begin{array}{l}\text { Health } \\
\text { status, IV }\end{array}$ \\
\hline
\end{tabular}




\begin{tabular}{|c|c|c|}
\hline 137 & Delport, S. D.,Becker, P. J.,Bergh, A. Growth, feeding practices and infections in black infants. S Afr Med J.1997;87:57-61. & IV, DV \\
\hline 138 & $\begin{array}{l}\text { Dewey, K. G.,Cohen, R. J.,Rivera, L. L.,Canahuati, J.,Brown, K. H. Do exclusively breast-fed infants require extra protein?. Pediatr } \\
\text { Res.1996;39:303-7. }\end{array}$ & IV \\
\hline 139 & $\begin{array}{l}\text { Dewey, K. G.,Hawck, M. G.,Brown, K. H.,Lartey, A.,Cohen, R. J.,Peerson, J. M. Infant weight-for-length is positively associated with } \\
\text { subsequent linear growth across four different populations. Matern Child Nutr.2005;1:11-20. }\end{array}$ & IV \\
\hline 140 & $\begin{array}{l}\text { Dewey, K. G.,Heinig, M. J.,Nommsen, L. A.,Lonnerdal, B. Adequacy of energy intake among breast-fed infants in the DARLING } \\
\text { study: relationships to growth velocity, morbidity, and activity levels. Davis Area Research on Lactation, Infant Nutrition and Growth. } \\
\text { J Pediatr.1991;119:538-47. }\end{array}$ & IV \\
\hline 141 & $\begin{array}{l}\text { Dewey, K. G.,Lonnerdal, B. Milk and nutrient intake of breast-fed infants from } 1 \text { to } 6 \text { months: relation to growth and fatness. J } \\
\text { Pediatr Gastroenterol Nutr.1983;2:497-506. }\end{array}$ & IV \\
\hline 142 & $\begin{array}{l}\text { Dewey, K. G.,Peerson, J. M.,Brown, K. H.,Krebs, N. F.,Michaelsen, K. F.,Persson, L. A.,Salmenpera, L.,Whitehead, R. G.,Yeung, D. } \\
\text { L. Growth of breast-fed infants deviates from current reference data: a pooled analysis of US, Canadian, and European data sets. } \\
\text { World Health Organization Working Group on Infant Growth. Pediatrics.1995;96:495-503. }\end{array}$ & Design \\
\hline 143 & $\begin{array}{l}\text { Dewey, K. G.,Peerson, J. M.,Heinig, M. J.,Nommsen, L. A.,Lonnerdal, B.,Lopez de Romana, G., de Kanashiro, H. C.,Black, R. } \\
\text { E.,Brown, K. H. Growth patterns of breast-fed infants in affluent (United States) and poor (Peru) communities: implications for timing } \\
\text { of complementary feeding. Am J Clin Nutr.1992;56:1012-8. }\end{array}$ & IV, DV \\
\hline 144 & Dewey, K. G. Complementary feeding and infant growth and body composition. Pediatrics.2000;106:1281. & Design \\
\hline 145 & $\begin{array}{l}\text { Dewey,K. G.,Cohen,R. J.,Brown,K. H.,Rivera,L. L. Effects of exclusive breastfeeding for four versus six months on maternal } \\
\text { nutritional status and infant motor development: results of two randomized trials in Honduras. J Nutr.2001;131:262-7. }\end{array}$ & Country \\
\hline 146 & $\begin{array}{l}\text { Dewey,K. G.,Cohen,R. J.,Rivera,L. L.,Brown,K. H. Effects of age of introduction of complementary foods on iron status of breast-fed } \\
\text { infants in Honduras. Am J Clin Nutr.1998;67:878-84. }\end{array}$ & DV \\
\hline 147 & $\begin{array}{l}\text { Dewey,K. G.,Huffman,S. L. Maternal, infant, and young child nutrition: combining efforts to maximize impacts on child growth and } \\
\text { micronutrient status. Food Nutr Bull.2009;30:S187-9. }\end{array}$ & Design \\
\hline 148 & $\begin{array}{l}\text { Dewey,K. G.,Romero-Abal,M. E.,Quan de Serrano,J.,Bulux,J.,Peerson,J. M.,Eagle,P.,Solomons,N. W. Effects of discontinuing } \\
\text { coffee intake on iron status of iron-deficient Guatemalan toddlers: a randomized intervention study. Am J Clin Nutr.1997;66:168-76. }\end{array}$ & DV \\
\hline 149 & $\begin{array}{l}\text { Diaz, S.,Herreros, C.,Aravena, R.,Casado, M. E., Reyes, M. V.,Schiappacasse, V. Breast-feeding duration and growth of fully breast- } \\
\text { fed infants in a poor urban Chilean population. Am J Clin Nutr.1995;62:371-6. }\end{array}$ & IV, DV \\
\hline 150 & $\begin{array}{l}\text { Doak, C. M.,van der Starre, R. E.,van Beusekom, I.,Campos Ponce, M., Vossenaar, M.,Solomons, N. W. Earlier introduction of } \\
\text { aguitas is associated with higher risk of stunting in infants and toddlers in the Western Highlands of Guatemala. Am J Clin } \\
\text { Nutr.2013;97:631-6. }\end{array}$ & Design \\
\hline 151 & $\begin{array}{l}\text { Domellof,M.,Cohen,R. J.,Dewey,K. G.,Hernell,O.,Rivera,L. L.,Lonnerdal,B. Iron supplementation of breast-fed Honduran and } \\
\text { Swedish infants from } 4 \text { to } 9 \text { months of age. J Pediatr.2001;138:679-87. }\end{array}$ & IV, DV \\
\hline 152 & $\begin{array}{l}\text { Dong,C.,Ge,P.,Ren,X.,Wang,J.,Fan,H.,Yan,X.,Yin,S. A. Prospective study on the effectiveness of complementary food supplements } \\
\text { on improving status of elder infants and young children in the areas affected by Wenchuan earthquake. PLoS One.2013;8:e72711. }\end{array}$ & $\begin{array}{l}\text { Design, } \\
\text { Health } \\
\text { status }\end{array}$ \\
\hline
\end{tabular}




\begin{tabular}{|c|c|c|}
\hline 153 & Donma, M. M.,Donma, O. Infant feeding and growth: a study on Turkish infants from birth to 6 months. Pediatr Int.1999;41:542-8. & IV \\
\hline 154 & $\begin{array}{l}\text { Donma, M. M.,Donma, O. The influence of feeding patterns on head circumference among Turkish infants during the first } 6 \text { months } \\
\text { of life. Brain Dev.1997;19:393-7. }\end{array}$ & IV \\
\hline 155 & $\begin{array}{l}\text { Dubakiene, R.,Rudzeviciene, O.,Butiene, I.,Sezaite, I.,Petronyte, M.,Vaicekauskaite, D.,Zvirbliene, A. Studies on early allergic } \\
\text { sensitization in the Lithuanian birth cohort. ScientificWorldJournal.2012;2012:909524. }\end{array}$ & IV, DV \\
\hline 156 & $\begin{array}{l}\text { Dubois, L.,Girard, M. Breast-feeding, day-care attendance and the frequency of antibiotic treatments from } 1.5 \text { to } 5 \text { years: a } \\
\text { population-based longitudinal study in Canada. Soc Sci Med.2005;60:2035-44. }\end{array}$ & IV, DV \\
\hline 157 & $\begin{array}{l}\text { Dubois, L., Girard, M. Social inequalities in infant feeding during the first year of life. The Longitudinal Study of Child Development in } \\
\text { Quebec (LSCDQ 1998-2002). Public Health Nutr.2003;6:773-83. }\end{array}$ & IV, DV \\
\hline 158 & $\begin{array}{l}\text { Dubowitz, T.,Levinson, D.,Peterman, J. N.,Verma, G.,Jacob, S.,Schultink, W. Intensifying efforts to reduce child malnutrition in India: } \\
\text { an evaluation of the Dular program in Jharkhand, India. Food Nutr Bull.2007;28:266-73. }\end{array}$ & IV \\
\hline 159 & $\begin{array}{l}\text { Duggan,C.,Penny,M. E.,Hibberd,P.,Gil,A.,Huapaya,A.,Cooper,A.,Coletta,F.,Emenhiser,C.,Kleinman,R. E. Oligofructose- } \\
\text { supplemented infant cereal: } 2 \text { randomized, blinded, community-based trials in Peruvian infants. Am J Clin Nutr.2003;77:937-42. }\end{array}$ & IV \\
\hline 160 & Dunne,A. Nutrition in infancy: achieving nutrition needs for new mothers and children. Br J Community Nurs.2012;Suppl:S22. & Design \\
\hline 161 & Edmondson, L. What toddlers eat really matters. J Fam Health Care.2011;21:33-41. & Design \\
\hline 162 & $\begin{array}{l}\text { El-Sayed H,Martines J,Rakha M,Zekry O,Abdel-Hak M,Abbas H. The effectiveness of the WHO training course on complementary } \\
\text { feeding counseling in a primary care setting, Ismailia, Egypt. J Egypt Public Health Assoc.2014;89:1-8. }\end{array}$ & IV \\
\hline 163 & $\begin{array}{l}\text { Elwood, P. C.,Haley, T. J.,Hughes, S. J.,Sweetnam, P. M.,Gray, O. P.,Davies, D. P. Child growth (0-5 years), and the effect of } \\
\text { entitlement to a milk supplement. Arch Dis Child.1981;56:831-5. }\end{array}$ & IV \\
\hline 164 & $\begin{array}{l}\text { Emmett,P. M.,Jones,L. R. Diet and growth in infancy: relationship to socioeconomic background and to health and development in } \\
\text { the Avon Longitudinal Study of Parents and Children. Nutr Rev.2014;72:483-506. }\end{array}$ & Design \\
\hline 165 & $\begin{array}{l}\text { Emond A,Drewett R,Blair P,Emmett P. Postnatal factors associated with failure to thrive in term infants in the Avon Longitudinal } \\
\text { Study of Parents and Children. Arch Dis Child.2007;92:115-9. }\end{array}$ & IV \\
\hline 166 & $\begin{array}{l}\text { Eneroth,H.,El Arifeen,S.,Persson,L. A.,Kabir,I.,Lonnerdal,B.,Hossain,M. B.,Ekstrom,E. C. Duration of exclusive breast-feeding and } \\
\text { infant iron and zinc status in rural Bangladesh. J Nutr.2009;139:1562-7. }\end{array}$ & IV, DV \\
\hline 167 & $\begin{array}{l}\text { Engelmann,M. D.,Davidsson,L.,Sandstrom,B.,Walczyk,T.,Hurrell,R. F.,Michaelsen,K. F. The influence of meat on nonheme iron } \\
\text { absorption in infants. Pediatr Res.1998;43:768-73. }\end{array}$ & DV \\
\hline 168 & $\begin{array}{l}\text { England, L.,Brenner, R.,Bhaskar, B.,Simons-Morton, B.,Das, A.,Revenis, M.,Mehta, N.,Clemens, J. Breastfeeding practices in a } \\
\text { cohort of inner-city women: the role of contraindications. BMC Public Health.2003;3:28. }\end{array}$ & IV, DV \\
\hline 169 & $\begin{array}{l}\text { Escribano, J.,Luque, V.,Ferre, N.,Mendez-Riera, G.,Koletzko, B.,Grote, V.,Demmelmair, H.,Bluck, L.,Wright, A.,Closa-Monasterolo, } \\
\text { R. Effect of protein intake and weight gain velocity on body fat mass at } 6 \text { months of age: the EU Childhood Obesity Programme. Int } \\
\text { J Obes (Lond).2012;36:548-53. }\end{array}$ & IV \\
\hline 170 & $\begin{array}{l}\text { Esfarjani, F.,Azar, M. R.,Gafarpour, M. IDDM and early exposure of infant to cow's milk and solid food. Indian J } \\
\text { Pediatr.2001;68:107-10. }\end{array}$ & DV \\
\hline
\end{tabular}




\begin{tabular}{|c|c|c|}
\hline 171 & Evans, D.,Hansen, J. D.,Moodie, A. D.,van der Spuy, H. I. Intellectual development and nutrition. J Pediatr.1980;97:358-63. & $\begin{array}{l}\text { Health } \\
\text { status, IV }\end{array}$ \\
\hline 172 & $\begin{array}{l}\text { Exl, B. M.,Deland, U.,Secretin, M. C.,Preysch, U.,Wall, M.,Shmerling, D. H. Improved general health status in an unselected infant } \\
\text { population following an allergen reduced dietary intervention programme. The ZUFF-study-programme. Part I: Study design and 6- } \\
\text { month nutritional behaviour. Eur J Nutr.2000;39:89-102. }\end{array}$ & DV \\
\hline 173 & $\begin{array}{l}\text { Exl, B. M.,Deland, U.,Secretin, M. C.,Preysch, U.,Wall, M.,Shmerling, D. H. Improved general health status in an unselected infant } \\
\text { population following an allergen-reduced dietary intervention programme: the ZUFF-STUDY-PROGRAMME. Part II: infant growth } \\
\text { and health status to age } 6 \text { months. ZUg-FrauenFeld. Eur J Nutr.2000;39:145-56. }\end{array}$ & IV \\
\hline 174 & $\begin{array}{l}\text { Faber,M.,Kvalsvig,J. D.,Lombard,C. J.,Benade,A. J. Effect of a fortified maize-meal porridge on anemia, micronutrient status, and } \\
\text { motor development of infants. Am J Clin Nutr.2005;82:1032-9. }\end{array}$ & Country \\
\hline 175 & $\begin{array}{l}\text { Faber,M. Dietary intake and anthropometric status differ for anaemic and non-anaemic rural South African infants aged 6-12 } \\
\text { months. J Health Popul Nutr.2007;25:285-93. }\end{array}$ & Design \\
\hline 176 & $\begin{array}{l}\text { Fabiansen, C.,Phelan, K. P.,Cichon, B.,Ritz, C.,Briend, A.,Michaelsen, K. F.,Friis, H.,Shepherd, S. Short children with a low } \\
\text { midupper arm circumference respond to food supplementation: an observational study from Burkina Faso. Am J Clin } \\
\text { Nutr.2016;103:415-21. }\end{array}$ & $\begin{array}{l}\text { Health } \\
\text { status }\end{array}$ \\
\hline 177 & $\begin{array}{l}\text { Fabius, R. J.,Merritt, R. J.,Fleiss, P. M.,Ashley, J. M. Malnutrition associated with a formula of barley water, corn syrup, and whole } \\
\text { milk. Am J Dis Child.1981;135:615-7. }\end{array}$ & Design \\
\hline 178 & Falkner, F. Introduction of food to infants. Genetic-environmental considerations. Am J Clin Nutr.1985;41:436-9. & Design \\
\hline 179 & $\begin{array}{l}\text { Fall CH,Borja JB,Osmond C,Richter L,Bhargava SK,Martorell R,Stein AD,Barros FC,Victora CG. Infant-feeding patterns and } \\
\text { cardiovascular risk factors in young adulthood: data from five cohorts in low- and middle-income countries. Int } J \\
\text { Epidemiol.2011;40:47-62. }\end{array}$ & Design \\
\hline 180 & $\begin{array}{l}\text { Farris, R. P.,Frank, G. C.,Webber, L. S.,Srinivasan, S. R.,Berenson, G. S. Influence of milk source on serum lipids and lipoproteins } \\
\text { during the first year of life, Bogalusa heart study. Am J Clin Nutr.1982;35:42-9. }\end{array}$ & IV \\
\hline 181 & Farrow, C. V.,Blissett, J. Controlling feeding practices: cause or consequence of early child weight?. Pediatrics.2008;121:e164-9. & IV \\
\hline 182 & Farrow, C.,Blissett, J. Does maternal control during feeding moderate early infant weight gain?. Pediatrics.2006;118:e293-8. & IV \\
\hline 183 & Fawcett, J. N. Feeding from birth to 18 months. Nursing (Lond).1981:956-8. & Design \\
\hline 184 & $\begin{array}{l}\text { Feldens CA, Vitolo MR, Rauber F,Cruz LN,Hilgert JB. Risk factors for discontinuing breastfeeding in southern Brazil: a survival } \\
\text { analysis. Matern Child Health J.2012;16:1257-65. }\end{array}$ & IV, DV \\
\hline 185 & $\begin{array}{l}\text { Feldens, C. A.,Ardenghi, T. M.,Cruz, L. N.,Scalco, G. P.,Vitolo, M. R. Advising mothers about breastfeeding and weaning reduced } \\
\text { pacifier use in the first year of life: a randomized trial. Community Dent Oral Epidemiol.2013;41:317-26. }\end{array}$ & IV, DV \\
\hline 186 & $\begin{array}{l}\text { Feldens, C. A.,Kramer, P. F., Sequeira, M. C., Rodrigues, P. H.,Vitolo, M. R. Maternal education is an independent determinant of } \\
\text { cariogenic feeding practices in the first year of life. Eur Arch Paediatr Dent.2012;13:70-5. }\end{array}$ & DV \\
\hline 187 & Fergusson, D. M.,Woodward, L. J. Breast feeding and later psychosocial adjustment. Paediatr Perinat Epidemiol.1999;13:144-57. & IV \\
\hline
\end{tabular}




\begin{tabular}{|c|c|c|}
\hline 188 & $\begin{array}{l}\text { Fernandes, T. A.,Werneck, G. L.,Hasselmann, M. H. Prepregnancy weight, weight gain during pregnancy, and exclusive } \\
\text { breastfeeding in the first month of Life in Rio de Janeiro, Brazil. J Hum Lact.2012;28:55-61. }\end{array}$ & IV, DV \\
\hline 189 & $\begin{array}{l}\text { Fernstrom, J. D.,Navia, J. L. Introduction to the workshop. Low-calorie sweeteners and weight control--what the science tells us. J } \\
\text { Nutr.2012;142:1132s-3s. }\end{array}$ & Design \\
\hline 190 & $\begin{array}{l}\text { Ferrara,M.,Bertocco,F.,Ricciardi,A.,Ferrara,D.,Incarnato,L.,Capozzi,L. Iron deficiency screening in the first three years of life: a } \\
\text { three-decade-long retrospective case study. Hematology.2014;19:239-43. }\end{array}$ & DV \\
\hline 191 & $\begin{array}{l}\text { Fildes, A.,van Jaarsveld, C. H.,Llewellyn, C.,Wardle, J.,Fisher, A. Parental control over feeding in infancy. Influence of infant weight, } \\
\text { appetite and feeding method. Appetite.2015;91:101-6. }\end{array}$ & IV, DV \\
\hline 192 & $\begin{array}{l}\text { Fisher, J. O.,Birch, L. L.,Smiciklas-Wright, H.,Picciano, M. F. Breast-feeding through the first year predicts maternal control in } \\
\text { feeding and subsequent toddler energy intakes. J Am Diet Assoc.2000;100:641-6. }\end{array}$ & IV, DV \\
\hline 193 & $\begin{array}{l}\text { Flax, V. L.,Bentley, M. E.,Chasela, C. S.,Kayira, D.,Hudgens, M. G.,Kacheche, K. Z.,Chavula, C.,Kourtis, A. P.,Jamieson, D. J.,van } \\
\text { der Horst, C. M.,Adair, L. S. Lipid-based nutrient supplements are feasible as a breastmilk replacement for HIV-exposed infants from } \\
24 \text { to } 48 \text { weeks of age. J Nutr.2013;143:701-7. }\end{array}$ & $\begin{array}{l}\text { Health } \\
\text { status, IV }\end{array}$ \\
\hline 194 & $\begin{array}{l}\text { Fomon SJ,Ziegler EE,Nelson SE,Edwards BB. Sweetness of diet and food consumption by infants. Proc Soc Exp Biol } \\
\text { Med.1983;173:190-3. }\end{array}$ & IV, DV \\
\hline 195 & $\begin{array}{l}\text { Fomon, S. J.,Rogers, R. R.,Ziegler, E. E.,Nelson, S. E.,Thomas, L. N. Indices of fatness and serum cholesterol at age eight years in } \\
\text { relation to feeding and growth during early infancy. Pediatr Res.1984;18:1233-8. }\end{array}$ & IV, Date \\
\hline 196 & $\begin{array}{l}\text { Forns,J.,Torrent,M.,Garcia-Esteban,R.,Caceres,A.,Pilar Gomila,M.,Martinez,D.,Morales,E.,Julvez,J.,Grimalt,J. O.,Sunyer,J. } \\
\text { Longitudinal association between early life socio-environmental factors and attention function at the age } 11 \text { years. Environ } \\
\text { Res.2012;117:54-9. }\end{array}$ & IV \\
\hline 197 & $\begin{array}{l}\text { Foroushani, A. R.,Mohammad, K.,Mahmoodi, M.,Siassi, F. Effect of breastfeeding on cognitive performance in a British birth cohort. } \\
\text { East Mediterr Health J.2010;16:202-8. }\end{array}$ & IV \\
\hline 198 & $\begin{array}{l}\text { Franks, A. J.,Jurgensen, C. Nutrition and health in the first year of life on a Pacific atoll. Observations on Abemama Atoll, Central } \\
\text { Pacific. Trans R Soc Trop Med Hyg.1985;79:681-4. }\end{array}$ & IV, DV \\
\hline 199 & $\begin{array}{l}\text { Frederiksen,B. N.,Seifert,J.,Kroehl,M.,Lamb,M. M.,Milne,G. L.,Rewers,M.,Norris,J. M. Timing of solid food introduction is associated } \\
\text { with urinary F2-isoprostane concentrations in childhood. Pediatric Research.2015;78:451-456. }\end{array}$ & DV \\
\hline 200 & $\begin{array}{l}\text { Freeman, V.,van't Hof, M.,Haschke, F. Patterns of milk and food intake in infants from birth to age } 36 \text { months: the Euro-growth } \\
\text { study. J Pediatr Gastroenterol Nutr.2000;31 Suppl 1:S76-85. }\end{array}$ & DV \\
\hline 201 & $\begin{array}{l}\text { Freeman,V. E.,Mulder,J.,van't Hof,M. A.,Hoey,H. M.,Gibney,M. J. A longitudinal study of iron status in children at } 12,24 \text { and } 36 \\
\text { months. Public Health Nutr.1998;1:93-100. }\end{array}$ & DV \\
\hline 202 & $\begin{array}{l}\text { Friel,J. K.,Andrews,W. L.,Simmons,B. S.,L'Abbe,M. R.,Mercer,C.,MacDonald,A.,McCloy,U. R. Evaluation of full-term infants fed an } \\
\text { evaporated milk formula. Acta Paediatr.1997;86:448-53. }\end{array}$ & IV \\
\hline 203 & $\begin{array}{l}\text { Froozani, M. D.,Malekafzali, H.,Bahrini, B. Growth of a group of low income infants in the first year of life. J Trop } \\
\text { Pediatr.1980;26:96-8. }\end{array}$ & Design, IV \\
\hline 204 & $\begin{array}{l}\text { Fuchs lii, G. J.,Gastanaduy, A. S.,Suskind, R. M. Comparative metabolic study of older infants fed infant formula, transition formula, } \\
\text { or whole cow's milk. Nutrition Research.1992;12:1467-1478. }\end{array}$ & IV \\
\hline 205 & Fuchs, G. J.,Farris, R. P.,DeWier, M.,Hutchinson, S.,Strada, R.,Suskind, R. M. Effect of dietary fat on cardiovascular risk factors in & IV \\
\hline
\end{tabular}




\begin{tabular}{|c|c|c|}
\hline & infancy. Pediatrics.1994;93:756-63. & \\
\hline 206 & $\begin{array}{l}\text { Gabriel, C. G.,Corso, A. C.,Caldeira, G. V.,Gimeno, S. G.,Schmitz Bde, A.,de Vasconcelos Fde, A. Overweight and obesity related } \\
\text { factors in schoolchildren in Santa Catarina State, Brazil. Arch Latinoam Nutr.2010;60:332-9. }\end{array}$ & Design \\
\hline 207 & $\begin{array}{l}\text { Gaffney, K. F.,Brito, A. V.,Kitsantas, P.,Kermer, D. A. Early Feeding Practices and Weight Status at One Year of Age: A Comparison } \\
\text { of Hispanic Immigrant Mother-Infant Dyads with Participants of the Infant Feeding Practices Study II. Child Obes.2016. }\end{array}$ & IV, DV \\
\hline 208 & $\begin{array}{l}\text { Gale, C., Thomas, E. L.,Jeffries, S.,Durighel, G.,Logan, K. M.,Parkinson, J. R.,Uthaya, S.,Santhakumaran, S.,Bell, J. D.,Modi, N. } \\
\text { Adiposity and hepatic lipid in healthy full-term, breastfed, and formula-fed human infants: a prospective short-term longitudinal cohort } \\
\text { study. Am J Clin Nutr.2014;99:1034-40. }\end{array}$ & IV \\
\hline 209 & $\begin{array}{l}\text { Galler, J. R., Ramsey, F. C.,Harrison, R. H.,Brooks, R.,Weiskopf-Bock, S. Infant feeding practices in Barbados predict later growth. J } \\
\text { Nutr.1998;128:1328-35. }\end{array}$ & IV \\
\hline 210 & $\begin{array}{l}\text { Gallo PV. Physiological and behavioral consequences of maternal and post-weaning protein restriction. Physiol Behav.1981;26:77- } \\
84 .\end{array}$ & $\begin{array}{l}\text { Design, } \\
\text { Health } \\
\text { status }\end{array}$ \\
\hline 211 & $\begin{array}{l}\text { Galtry, J. Punching above its weight: does New Zealand's responsibility for protecting, promoting, and supporting breastfeeding } \\
\text { extend beyond its own borders?. J Hum Lact.2013;29:128-31. }\end{array}$ & Design \\
\hline 212 & $\begin{array}{l}\text { Garza, C. The INTERGROWTH-21st project and the multicenter growth reference study: enhanced opportunities for monitoring } \\
\text { growth from early pregnancy to } 5 \text { years of age. Breastfeed Med.2014;9:341-4. }\end{array}$ & Design \\
\hline 213 & $\begin{array}{l}\text { Geuns, M.,Huisinga, C.,van Staveren, W. A.,Deurenberg, P.,Hautvast, J. G. Intakes of energy, protein and calcium and weaning } \\
\text { pattern of a group of Dutch infants aged 6-12 months. Tijdschr Kindergeneeskd.1985;53:50-6. }\end{array}$ & $\begin{array}{l}\text { Design, IV, } \\
\text { DV }\end{array}$ \\
\hline 214 & $\begin{array}{l}\text { Ghorashi,Z.,Nezami,N.,Ghalehgolab Behbahan,A.,Ghorashi,S. Supplemental food may not prevent iron-deficiency anemia in } \\
\text { infants. Indian J Pediatr.2008;75:1121-4. }\end{array}$ & DV \\
\hline 215 & $\begin{array}{l}\text { Ghosh,S.,Kilaru,A.,Ganapathy,S. Nutrition education and infant growth in rural Indian infants: narrowing the gender gap?. J Indian } \\
\text { Med Assoc.2002;100:483-4, 486-8, 490. }\end{array}$ & IV \\
\hline 216 & $\begin{array}{l}\text { Gianni, M. L., Roggero, P.,Morlacchi, L.,Garavaglia, E.,Piemontese, P.,Mosca, F. Formula-fed infants have significantly higher fat- } \\
\text { free mass content in their bodies than breastfed babies. Acta Paediatr.2014;103:e277-81. }\end{array}$ & IV \\
\hline 217 & Gibson,R. S.,Hotz,C. The adequacy of micronutrients in complementary foods. Pediatrics.2000;106:1298-9. & Design \\
\hline 218 & $\begin{array}{l}\text { Gill, D. G.,Vincent, S.,Segal, D. S. Follow-on formula in the prevention of iron deficiency: a multicentre study. Acta } \\
\text { Paediatr.1997;86:683-9. }\end{array}$ & IV \\
\hline 219 & $\begin{array}{l}\text { Gillman, M. W.,Rifas-Shiman, S. L.,Camargo, C. A., Jr.,Berkey, C. S.,Frazier, A. L.,Rockett, H. R.,Field, A. E.,Colditz, G. A. Risk of } \\
\text { overweight among adolescents who were breastfed as infants. Jama.2001;285:2461-7. }\end{array}$ & Design \\
\hline 220 & $\begin{array}{l}\text { Gokcay, G.,Turan, J. M.,Partalci, A.,Neyzi, O. Growth of infants during the first year of life according to feeding regimen in the first } 4 \\
\text { months. J Trop Pediatr.2003;49:6-12. }\end{array}$ & IV \\
\hline 221 & $\begin{array}{l}\text { Golley,R. K.,Smithers,L. G.,Mittinty,M. N.,Brazionis,L.,Emmett,P.,Northstone,K.,Campbell,K.,McNaughton,S. A.,Lynch,J. W. An } \\
\text { index measuring adherence to complementary feeding guidelines has convergent validity as a measure of infant diet quality. J } \\
\text { Nutr.2012;142:901-8. }\end{array}$ & IV, DV \\
\hline
\end{tabular}




\begin{tabular}{|c|c|c|}
\hline 222 & $\begin{array}{l}\text { Gomez Santos, S. F.,Estevez Santiago, R.,Palacios Gil-Antunano, N.,Leis Trabazo, M. R.,Tojo Sierra, R.,Cuadrado Vives, } \\
\text { C.,Beltran de Miguel, B.,Avila Torres, J. M.,Varela Moreiras, G.,Casas Esteve, R. THAO-CHILD HEALTH PROGRAMME: } \\
\text { COMMUNITY BASED INTERVENTION FOR HEALTHY LIFESTYLES PROMOTION TO CHILDREN AND FAMILIES: RESULTS } \\
\text { OF A COHORT STUDY. Nutr Hosp.2015;32:2584-7. }\end{array}$ & Age \\
\hline 223 & $\begin{array}{l}\text { Gomez-Sanchiz, M.,Canete, R.,Rodero, I.,Baeza, J. E.,Avila, O. Influence of breast-feeding on mental and psychomotor } \\
\text { development. Clin Pediatr (Phila).2003;42:35-42. }\end{array}$ & IV \\
\hline 224 & $\begin{array}{l}\text { Gomez-Sanchiz, M.,Canete, R.,Rodero, I.,Baeza, J. E.,Gonzalez, J. A. Influence of breast-feeding and parental intelligence on } \\
\text { cognitive development in the 24-month-old child. Clin Pediatr (Phila).2004;43:753-61. }\end{array}$ & IV \\
\hline 225 & $\begin{array}{l}\text { Gong, Y. H.,Ji, C. Y.,Zheng, X. X.,Shan, J. P.,Hou, R. Correlation of 4-month infant feeding modes with their growth and iron status } \\
\text { in Beijing. Chin Med J (Engl).2008;121:392-8. }\end{array}$ & IV \\
\hline 226 & $\begin{array}{l}\text { Gooze, R. A.,Anderson, S. E.,Whitaker, R. C. Prolonged bottle use and obesity at } 5.5 \text { years of age in US children. J } \\
\text { Pediatr.2011;159:431-6. }\end{array}$ & IV \\
\hline 227 & $\begin{array}{l}\text { Greene, L. C.,Lucas, A.,Livingstone, M. B.,Harland, P. S.,Baker, B. A. Relationship between early diet and subsequent cognitive } \\
\text { performance during adolescence. Biochem Soc Trans.1995;23:376s. }\end{array}$ & Design, IV \\
\hline 228 & $\begin{array}{l}\text { Greer,F. R.,Searcy,J. E.,Levin,R. S.,Steichen,J. J.,Steichen-Asche,P. S.,Tsang,R. C. Bone mineral content and serum 25- } \\
\text { hydroxyvitamin D concentrations in breast-fed infants with and without supplemental vitamin D: one-year follow-up. J } \\
\text { Pediatr.1982;100:919-22. }\end{array}$ & IV \\
\hline 229 & $\begin{array}{l}\text { Grote, V.,von Kries, R.,Closa-Monasterolo, R.,Scaglioni, S.,Gruszfeld, D.,Sengier, A., Langhendries, J. P.,Koletzko, B. Protein intake } \\
\text { and growth in the first } 24 \text { months of life. J Pediatr Gastroenterol Nutr.2010;51 Suppl 3:S117-8. }\end{array}$ & Design, IV \\
\hline 230 & $\begin{array}{l}\text { Gruszfeld, D.,Weber, M.,Nowakowska-Rysz, M.,Janas, R.,Kozlik-Feldmann, R.,Xhonneux, A.,Carlier, C.,Riva, E.,Verduci, E.,Closa- } \\
\text { Monasterolo, R., Escribano, J.,Dobrzanska, A.,Koletzko, B. Protein intake in infancy and carotid intima media thickness at } 5 \text { years--a } \\
\text { secondary analysis from a randomized trial. Ann Nutr Metab.2015;66:51-9. }\end{array}$ & IV \\
\hline 231 & $\begin{array}{l}\text { Gubbels, J. S., Thijs, C.,Stafleu, A.,van Buuren, S.,Kremers, S. P. Association of breast-feeding and feeding on demand with child } \\
\text { weight status up to } 4 \text { years. Int J Pediatr Obes.2011;6:e515-22. }\end{array}$ & IV, DV \\
\hline 232 & $\begin{array}{l}\text { Gunderson, E. P.,Hurston, S. R.,Dewey, K. G.,Faith, M. S.,Charvat-Aguilar, N.,Khoury, V. C.,Nguyen, V. T., Quesenberry, C. P., Jr. } \\
\text { The study of women, infant feeding and type } 2 \text { diabetes after GDM pregnancy and growth of their offspring (SWIFT Offspring study): } \\
\text { prospective design, methodology and baseline characteristics. BMC Pregnancy Childbirth.2015;15:150. }\end{array}$ & Design \\
\hline 233 & $\begin{array}{l}\text { Gunnarsdottir I,Schack-Nielsen L,Michaelsen KF,Sorensen TI,Thorsdottir I. Infant weight gain, duration of exclusive breast-feeding } \\
\text { and childhood BMI - two similar follow-up cohorts. Public Health Nutr.2010;13:201-7. }\end{array}$ & IV \\
\hline 234 & $\begin{array}{l}\text { Gunnarsdottir, I.,Thorsdottir, I. Relationship between growth and feeding in infancy and body mass index at the age of } 6 \text { years. Int } J \\
\text { Obes Relat Metab Disord.2003;27:1523-7. }\end{array}$ & IV \\
\hline 235 & $\begin{array}{l}\text { Gunther, A. L.,Buyken, A. E.,Kroke, A. Protein intake during the period of complementary feeding and early childhood and the } \\
\text { association with body mass index and percentage body fat at } 7 \text { y of age. Am J Clin Nutr.2007;85:1626-33. }\end{array}$ & IV \\
\hline 236 & $\begin{array}{l}\text { Gunther, A. L.,Buyken, A. E.,Kroke, A. The influence of habitual protein intake in early childhood on BMI and age at adiposity } \\
\text { rebound: results from the DONALD Study. Int J Obes (Lond).2006;30:1072-9. }\end{array}$ & IV \\
\hline 237 & $\begin{array}{l}\text { Gurnida, D. A., Rowan, A. M.,Idjradinata, P.,Muchtadi, D.,Sekarwana, N. Association of complex lipids containing gangliosides with } \\
\text { cognitive development of 6-month-old infants. Early Hum Dev.2012;88:595-601. }\end{array}$ & IV \\
\hline
\end{tabular}




\begin{tabular}{|c|c|c|}
\hline 238 & $\begin{array}{l}\text { Hamilton, K.,Daniels, L.,White, K. M.,Murray, N.,Walsh, A. Predicting mothers' decisions to introduce complementary feeding at } 6 \\
\text { months. An investigation using an extended theory of planned behaviour. Appetite.2011;56:674-81. }\end{array}$ & IV, DV \\
\hline 239 & $\begin{array}{l}\text { Hammer, L. D.,Bryson, S.,Agras, W. S. Development of feeding practices during the first } 5 \text { years of life. Arch Pediatr Adolesc } \\
\text { Med.1999;153:189-94. }\end{array}$ & DV \\
\hline 240 & $\begin{array}{l}\text { Han,Y. H.,Yon,M.,Han,H. S.,Johnston,K. E.,Tamura,T.,Hyun,T. Zinc status and growth of Korean infants fed human milk, casein- } \\
\text { based, or soy-based formula: three-year longitudinal study. Nutr Res Pract.2011;5:46-51. }\end{array}$ & IV \\
\hline 241 & $\begin{array}{l}\text { Harbild, H. L.,Harslof, L. B.,Christensen, J. H.,Kannass, K. N.,Lauritzen, L. Fish oil-supplementation from } 9 \text { to } 12 \text { months of age } \\
\text { affects infant attention in a free-play test and is related to change in blood pressure. Prostaglandins Leukot Essent Fatty } \\
\text { Acids.2013;89:327-33. }\end{array}$ & IV \\
\hline 242 & $\begin{array}{l}\text { Harit D,Faridi MM,Aggarwal A,Sharma SB. Lipid profile of term infants on exclusive breastfeeding and mixed feeding: a comparative } \\
\text { study. Eur J Clin Nutr.2008;62:203-9. }\end{array}$ & IV \\
\hline 243 & $\begin{array}{l}\text { Harrington,M.,Hotz,C.,Zeder,C.,Polvo,G. O.,Villalpando,S.,Zimmermann,M. B.,Walczyk,T.,Rivera,J. A.,Hurrell,R. F. A comparison of } \\
\text { the bioavailability of ferrous fumarate and ferrous sulfate in non-anemic Mexican women and children consuming a sweetened } \\
\text { maize and milk drink. Eur J Clin Nutr.2011;65:20-5. }\end{array}$ & IV, DV \\
\hline 244 & Harris,R. Inadequate diets: short- and long-term problems. J Fam Health Care.2002;12:6. & Design \\
\hline 245 & $\begin{array}{l}\text { Hasan,J.,Ray,J.,Khan,Z. Role of weaning in the nutritional status of infant--a longitudinal study in the rural area of Aligarh. J Indian } \\
\text { Med Assoc.1996;94:169, } 215 .\end{array}$ & $\begin{array}{l}\text { Design, IV, } \\
\text { DV }\end{array}$ \\
\hline 246 & $\begin{array}{l}\text { Haschke, F.,Pietschnig, B.,Vanura, H.,Heil, M.,Steffan, I.,Hobiger, G.,Schuster, E.,Camaya, Z. Iron intake and iron nutritional status } \\
\text { of infants fed iron-fortified beikost with meat. Am J Clin Nutr.1988;47:108-12. }\end{array}$ & IV \\
\hline 247 & $\begin{array}{l}\text { Hawley, N. L.,Johnson, W.,Nu'usolia, O.,McGarvey, S. T. The contribution of feeding mode to obesogenic growth trajectories in } \\
\text { American Samoan infants. Pediatr Obes.2014;9:e1-e13. }\end{array}$ & IV \\
\hline 248 & $\begin{array}{l}\text { Hay,G.,Johnston,C.,Whitelaw,A.,Trygg,K.,Refsum,H. Folate and cobalamin status in relation to breastfeeding and weaning in } \\
\text { healthy infants. Am J Clin Nutr.2008;88:105-14. }\end{array}$ & DV \\
\hline 249 & $\begin{array}{l}\text { Hediger, M. L.,Overpeck, M. D.,Ruan, W. J.,Troendle, J. F. Early infant feeding and growth status of US-born infants and children } \\
\text { aged 4-71 mo: analyses from the third National Health and Nutrition Examination Survey, 1988-1994. Am J Clin Nutr.2000;72:159- } \\
67 .\end{array}$ & Design, IV \\
\hline 250 & $\begin{array}{l}\text { Hegar, B.,Dewanti, N. R.,Kadim, M.,Alatas, S.,Firmansyah, A.,Vandenplas, Y. Natural evolution of regurgitation in healthy infants. } \\
\text { Acta Paediatr.2009;98:1189-93. }\end{array}$ & IV, DV \\
\hline 251 & $\begin{array}{l}\text { Heine, W.,Lapsien, C. Influence of early breast milk and formula feeding on body weight in children born in Rostock since } 1945 . \text { Bibl } \\
\text { Nutr Dieta.1982:17-8. }\end{array}$ & Design \\
\hline 252 & $\begin{array}{l}\text { Herbst, A.,Diethelm, K.,Cheng, G.,Alexy, U.,Icks, A.,Buyken, A. E. Direction of associations between added sugar intake in early } \\
\text { childhood and body mass index at age } 7 \text { years may depend on intake levels. J Nutr.2011;141:1348-54. }\end{array}$ & IV \\
\hline 253 & $\begin{array}{l}\text { Hess,S. Y.,Abbeddou,S.,Jimenez,E. Y.,Somé,J. W.,Vosti,S. A.,Ouédraogo,Z. P.,Guissou,R. M.,Ouédraogo,J. B.,Brown,K. H. Small- } \\
\text { quantity lipid-based nutrient supplements, regardless of their zinc content, increase growth and reduce the prevalence of stunting } \\
\text { and Wasting in young burkinabe children: A cluster-randomized trial. PLoS ONE.2015;10. }\end{array}$ & $\begin{array}{l}\text { IV, } \\
\text { Country }\end{array}$ \\
\hline 254 & $\begin{array}{l}\text { Hettiarachchi,M.,Lekamwasam,S.,Liyanage,C. Long-term cereal-based nutritional supplementation improved the total spine bone } \\
\text { mineral density amongst Sri Lankan preschool children: a randomized controlled study. J Pediatr Endocrinol Metab.2010;23:555-63. }\end{array}$ & IV, Age \\
\hline
\end{tabular}




\begin{tabular}{|c|c|c|}
\hline 255 & $\begin{array}{l}\text { Hijazi, S. S.,Abulaban, A.,Waterlow, J. C. The duration for which exclusive breast-feeding is adequate. A study in Jordan. Acta } \\
\text { Paediatr Scand.1989;78:23-8. }\end{array}$ & IV \\
\hline 256 & $\begin{array}{l}\text { Hillis, S. D.,Weigle, K.,Miranda, C. M.,McCann, M.,Bender, D.,Stewart, P. Supplementary feeding in Colombian child-care centers } \\
\text { and growth velocity. Pediatrics.1994;94:1066-9. }\end{array}$ & IV \\
\hline 257 & $\begin{array}{l}\text { Hitchcock, N. E.,Gracey, M.,Gilmour, A. I. The growth of breast fed and artificially fed infants from birth to twelve months. Acta } \\
\text { Paediatr Scand.1985;74:240-5. }\end{array}$ & IV \\
\hline 258 & Hitchcock, N. E.,McGuiness, D.,Gracey, M. Growth and feeding practices of Western Australian infants. Med J Aust.1982;1:372-6. & $\begin{array}{l}\text { Design, IV, } \\
\text { DV, Date }\end{array}$ \\
\hline 259 & Hitchcock, N. E.,Owles, E. N.,Gracey, M. Breast feeding and growth of healthy infants. Med J Aust.1981;2:536-7. & $\begin{array}{l}\text { IV, DV, } \\
\text { Date }\end{array}$ \\
\hline 260 & $\begin{array}{l}\text { Hoffman, D. R.,Garfield, S.,Morale, S. E.,Bosworth, R. G.,Castaneda, Y. S., Wheaton, D. K. H.,Theuer, R. C.,Birch, E. E. Visual and } \\
\text { neural development of breast-fed infants receiving docosahexaenoic acid (dha)-enriched baby food: a randomized clinical trial. } \\
\text { lovs.2004;45:ARVO E-abstract } 3510 \text {. }\end{array}$ & Design \\
\hline 261 & $\begin{array}{l}\text { Hokama,T. Levels of serum ferritin and total body iron among infants with different feeding regimens. Acta Paediatr } \\
\text { Jpn.1993;35:298-301. }\end{array}$ & IV, DV \\
\hline 262 & $\begin{array}{l}\text { Holgerson PL,Vestman NR,Claesson R,Ohman C,Domellof M,Tanner AC,Hernell O,Johansson I. Oral microbial profile discriminates } \\
\text { breast-fed from formula-fed infants. J Pediatr Gastroenterol Nutr.2013;56:127-36. }\end{array}$ & $\begin{array}{l}\text { Design, IV, } \\
\text { DV }\end{array}$ \\
\hline 263 & $\begin{array}{l}\text { Hop le, T.,Berger, J. Multiple micronutrient supplementation improves anemia, micronutrient nutrient status, and growth of } \\
\text { Vietnamese infants: double-blind, randomized, placebo-controlled trial. J Nutr.2005;135:660s-665s. }\end{array}$ & IV \\
\hline 264 & $\begin{array}{l}\text { Hop, L. T.,Gross, R.,Giay, T.,Sastroamidjojo, S.,Schultink, W.,Lang, N. T. Premature complementary feeding is associated with } \\
\text { poorer growth of vietnamese children. J Nutr.2000;130:2683-90. }\end{array}$ & Country \\
\hline 265 & $\begin{array}{l}\text { Hop, L. T.,Gross, R.,Giay, T.,Schultink, W.,Thuan, B. T.,Sastroamidjojo, S. Longitudinal observation of growth of Vietnamese } \\
\text { children in Hanoi, Vietnam from birth to } 10 \text { years of age. Eur J Clin Nutr.1997;51:164-71. }\end{array}$ & IV, DV \\
\hline 266 & $\begin{array}{l}\text { Hopkins, D.,Steer, C. D.,Northstone, K.,Emmett, P. M. Effects on childhood body habitus of feeding large volumes of cow or formula } \\
\text { milk compared with breastfeeding in the latter part of infancy. Am J Clin Nutr.2015;102:1096-103. }\end{array}$ & IV \\
\hline 267 & $\begin{array}{l}\text { Hopkins,D.,Emmett,P.,Steer,C., Rogers,I.,Noble,S.,Emond,A. Infant feeding in the second } 6 \text { months of life related to iron status: an } \\
\text { observational study. Arch Dis Child.2007;92:850-4. }\end{array}$ & DV \\
\hline 268 & $\begin{array}{l}\text { Hoppe, C.,Molgaard, C., Thomsen, B. L.,Juul, A.,Michaelsen, K. F. Protein intake at } 9 \text { mo of age is associated with body size but not } \\
\text { with body fat in 10-y-old Danish children. Am J Clin Nutr.2004;79:494-501. }\end{array}$ & IV \\
\hline 269 & $\begin{array}{l}\text { Horodynski, M. A.,Baker, S.,Van Egeren, L.,Olson, B.,Brophy-Herb, H.,Auld, G. The healthy babies curriculum. J Nutr Educ } \\
\text { Behav.2014;46:151-2. }\end{array}$ & Design, IV \\
\hline 270 & $\begin{array}{l}\text { Horta, B. L.,Bas, A.,Bhargava, S. K.,Fall, C. H.,Feranil, A.,de Kadt, J.,Martorell, R.,Richter, L. M.,Stein, A. D., Victora, C. G. Infant } \\
\text { feeding and school attainment in five cohorts from low- and middle-income countries. PLoS One.2013;8:e71548. }\end{array}$ & Design \\
\hline 271 & Horwood, L. J.,Fergusson, D. M. Breastfeeding and later cognitive and academic outcomes. Pediatrics.1998;101:E9. & IV \\
\hline 272 & $\begin{array}{l}\text { Hosaka, M.,Asayama, K.,Staessen, J. A.,Ohkubo, T.,Hayashi, K., Tatsuta, N.,Kurokawa, N.,Satoh, M.,Hashimoto, T.,Hirose, } \\
\text { T.,Obara, T.,Metoki, H.,Inoue, R.,Kikuya, M.,Nakai, K.,Imai, Y.,Satoh, H. Breastfeeding leads to lower blood pressure in 7-year-old } \\
\text { Japanese children: Tohoku Study of Child Development. Hypertens Res.2013;36:117-22. }\end{array}$ & IV, DV \\
\hline
\end{tabular}




\begin{tabular}{|c|c|c|}
\hline 273 & $\begin{array}{l}\text { Hudara S,Mimouni F, Rachman Y,Dayan B,Silbermintz A,Turner D. Retrospective cohort study of a new infant formula during the first } \\
6 \text { months of life: reflections on growth curves, human milk and formula feeding. Isr Med Assoc J.2010;12:676-80. }\end{array}$ & IV \\
\hline 274 & $\begin{array}{l}\text { Hurley, K. M.,Black, M. M. Introduction to a supplement on responsive feeding: promoting healthy growth and development for } \\
\text { infants and toddlers. J Nutr.2011;141:489. }\end{array}$ & Design \\
\hline 275 & $\begin{array}{l}\text { Husaini, M. A.,Karyadi, L.,Husaini, Y. K.,Sandjaja,,Karyadi, D.,Pollitt, E. Developmental effects of short-term supplementary feeding } \\
\text { in nutritionally-at-risk Indonesian infants. Am J Clin Nutr.1991;54:799-804. }\end{array}$ & Country \\
\hline 276 & $\begin{array}{l}\text { Inostroza, J.,Haschke, F.,Steenhout, P.,Grathwohl, D.,Nelson, S. E.,Ziegler, E. E. Low-protein formula slows weight gain in infants of } \\
\text { overweight mothers. J Pediatr Gastroenterol Nutr.2014;59:70-7. }\end{array}$ & IV \\
\hline 277 & $\begin{array}{l}\text { Isherwood,R. J.,Dimond,C.,Longhurst,S. Breast feeding and weaning practices in relation to nutritional status of under-5 children in } \\
\text { north Bangladesh. J Trop Pediatr.1988;34:28-31. }\end{array}$ & Design \\
\hline 278 & $\begin{array}{l}\text { Islam, M. M.,Peerson, J. M.,Ahmed, T.,Dewey, K. G.,Brown, K. H. Effects of varied energy density of complementary foods on } \\
\text { breast-milk intakes and total energy consumption by healthy, breastfed Bangladeshi children. Am J Clin Nutr.2006;83:851-8. }\end{array}$ & IV, DV \\
\hline 279 & $\begin{array}{l}\text { Jackson, D. A.,Imong, S. M.,Wongsawasdii, L.,Silprasert, A.,Preunglampoo, S.,Leelapat, P.,Drewett, R. F.,Amatayakul, K.,Baum, J. } \\
\text { D. Weaning practices and breast-feeding duration in Northern Thailand. Br J Nutr.1992;67:149-64. }\end{array}$ & IV, DV \\
\hline 280 & Jackson,M. I. Early childhood WIC participation, cognitive development and academic achievement. Soc Sci Med.2015;126:145-53. & IV \\
\hline 281 & $\begin{array}{l}\text { Jacobson, S. W.,Chiodo, L. M.,Jacobson, J. L. Breastfeeding effects on intelligence quotient in 4- and 11-year-old children. } \\
\text { Pediatrics.1999;103:e71. }\end{array}$ & IV \\
\hline 282 & $\begin{array}{l}\text { Jain,V.,Klein,B. P.,Nash,M.,Chapman-Novakofski,K. Two feasibility studies for introduction of multimicronutrient soy/whey-based } \\
\text { supplements in rural homes in Honduras. Journal of Hunger and Environmental Nutrition.2011;6:247-263. }\end{array}$ & IV \\
\hline 283 & Jansen,G.R.,O'B Hourihane. The nutritional status of preschool children in Egypt. World Rev Nutr Diet.1985;45:42-67. & Design \\
\hline 284 & $\begin{array}{l}\text { Jatanasen,A.,Sacholvicharn,V.,Ongkulna,P. An evaluation of supplementary feedings of Thai infants in urban slum communities: } \\
\text { effect on growth, health and nutritional status. J Med Assoc Thai.1983;66 Suppl 1:20-9. }\end{array}$ & $\begin{array}{l}\text { Design, } \\
\text { Health } \\
\text { status }\end{array}$ \\
\hline 285 & $\begin{array}{l}\text { Jensen, B. W.,Nichols, M.,Allender, S.,de Silva-Sanigorski, A.,Millar, L.,Kremer, P.,Lacy, K.,Swinburn, B. Inconsistent associations } \\
\text { between sweet drink intake and 2-year change in BMl among Victorian children and adolescents. Pediatr Obes.2013;8:271-83. }\end{array}$ & Age \\
\hline 286 & $\begin{array}{l}\text { Jensen, S. M.,Ritz, C.,Ejlerskov, K. T.,Molgaard, C.,Michaelsen, K. F. Infant BMI peak, breastfeeding, and body composition at age } \\
3 \text { y. Am J Clin Nutr.2015;101:319-25. }\end{array}$ & IV \\
\hline 287 & $\begin{array}{l}\text { Jensen,C. L.,Prager,T. C.,Zou,Y.,Fraley,J. K.,Maude,M.,Anderson,R. E.,Heird,W. C. Effects of maternal docosahexaenoic acid } \\
\text { supplementation on visual function and growth of breast-fed term infants. Lipids.1999;34 Suppl:S225. }\end{array}$ & Design, IV \\
\hline 288 & $\begin{array}{l}\text { Jones, G.,Hynes, K. L.,Dwyer, T. The association between breastfeeding, maternal smoking in utero, and birth weight with bone } \\
\text { mass and fractures in adolescents: a 16-year longitudinal study. Osteoporos Int.2013;24:1605-11. }\end{array}$ & IV \\
\hline 289 & $\begin{array}{l}\text { Jones, G.,Riley, M.,Dwyer, T. Breastfeeding in early life and bone mass in prepubertal children: a longitudinal study. Osteoporos } \\
\text { Int.2000;11:146-52. }\end{array}$ & IV \\
\hline 290 & $\begin{array}{l}\text { Jooste, P. L.,Rossouw, L. J.,Steenkamp, H. J.,Rossouw, J. E.,Swanepoel, A. S.,Charlton, D. O. Effect of breast feeding on the } \\
\text { plasma cholesterol and growth of infants. J Pediatr Gastroenterol Nutr.1991;13:139-42. }\end{array}$ & IV \\
\hline
\end{tabular}




\begin{tabular}{|c|c|c|}
\hline 291 & $\begin{array}{l}\text { Joseph, N.,Unnikrishnan, B.,Naik, V. A.,Mahantshetti, N. S.,Mallapur, M. D.,Kotian, S. M.,Nelliyanil, M. Infant rearing practices in } \\
\text { South India: a longitudinal study. J Family Med Prim Care.2013;2:37-43. }\end{array}$ & Country \\
\hline 292 & $\begin{array}{l}\text { Joshi N,Agho KE,Dibley MJ,Senarath U,Tiwari K. Determinants of inappropriate complementary feeding practices in young children } \\
\text { in Nepal: secondary data analysis of Demographic and Health Survey 2006. Matern Child Nutr.2012;8 Suppl 1:45-59. }\end{array}$ & $\begin{array}{l}\text { Design, } \\
\text { Country }\end{array}$ \\
\hline 293 & $\begin{array}{l}\text { Juez, G.,Diaz, S.,Casado, M. E.,Duran, E.,Salvatierra, A. M.,Peralta, O.,Croxatto, H. B. Growth pattern of selected urban Chilean } \\
\text { infants during exclusive breast-feeding. Am J Clin Nutr.1983;38:462-8. }\end{array}$ & IV \\
\hline 294 & $\begin{array}{l}\text { Jung, E.,Czajka-Narins, D. M. Birth weight doubling and tripling times: an updated look at the effects of birth weight, sex, race and } \\
\text { type of feeding. Am J Clin Nutr.1985;42:182-9. }\end{array}$ & IV \\
\hline 295 & $\begin{array}{l}\text { Kagura, J.,Feeley, A. B.,Micklesfield, L. K.,Pettifor, J. M.,Norris, S. A. Association between infant nutrition and anthropometry, and } \\
\text { pre-pubertal body composition in urban South African children. J Dev Orig Health Dis.2012;3:415-23. }\end{array}$ & Country \\
\hline 296 & $\begin{array}{l}\text { Kainonen E, Rautava S, Isolauri E. Immunological programming by breast milk creates an anti-inflammatory cytokine milieu in breast- } \\
\text { fed infants compared to formula-fed infants. Br J Nutr.2013;109:1962-70. }\end{array}$ & IV, DV \\
\hline 297 & $\begin{array}{l}\text { Kajantie, E.,Barker, D. J.,Osmond, C.,Forsen, T.,Eriksson, J. G. Growth before } 2 \text { years of age and serum lipids } 60 \text { years later: the } \\
\text { Helsinki Birth Cohort study. Int J Epidemiol.2008;37:280-9. }\end{array}$ & DV \\
\hline 298 & $\begin{array}{l}\text { Kallio, M. J.,Salmenpera, L.,Siimes, M. A.,Perheentupa, J.,Miettinen, T. A. Tracking of serum cholesterol and lipoprotein levels from } \\
\text { the first year of life. Pediatrics.1993;91:949-54. }\end{array}$ & IV, DV \\
\hline 299 & $\begin{array}{l}\text { Kallio,M. J.,Salmenpera,L.,Siimes,M. A.,Perheentupa,J.,Miettinen,T. A. Exclusive breast-feeding and weaning: effect on serum } \\
\text { cholesterol and lipoprotein concentrations in infants during the first year of life. Pediatrics.1992;89:663-6. }\end{array}$ & IV, DV \\
\hline 300 & $\begin{array}{l}\text { Karaolis-Danckert, N.,Buyken, A. E.,Kulig, M.,Kroke, A.,Forster, J.,Kamin, W.,Schuster, A.,Hornberg, C.,Keil, T.,Bergmann, R. } \\
\text { L.,Wahn, U.,Lau, S. How pre- and postnatal risk factors modify the effect of rapid weight gain in infancy and early childhood on } \\
\text { subsequent fat mass development: results from the Multicenter Allergy Study 90. Am J Clin Nutr.2008;87:1356-64. }\end{array}$ & IV \\
\hline 301 & $\begin{array}{l}\text { Karlsland Akeson, P. K.,Axelsson, I. E.,Raiha, N. C., Warm, A.,Minoli, I.,Moro, G. Protein intake and metabolism in formula-fed } \\
\text { infants given Swedish or Italian weaning foods. Acta Paediatr.2000;89:158-64. }\end{array}$ & IV \\
\hline 302 & $\begin{array}{l}\text { Karlsson Videhult, F.,Ohlund, I.,Stenlund, H.,Hernell, O.,West, C. E. Probiotics during weaning: a follow-up study on effects on body } \\
\text { composition and metabolic markers at school age. Eur J Nutr.2015;54:355-63. }\end{array}$ & IV \\
\hline 303 & $\begin{array}{l}\text { Kattelmann,K. K.,Ho,M.,Specker,B. L. Effect of timing of introduction of complementary foods on iron and zinc status of formula fed } \\
\text { infants at 12, 24, and } 36 \text { months of age. J Am Diet Assoc.2001;101:443-7. }\end{array}$ & DV \\
\hline 304 & $\begin{array}{l}\text { Kavanagh, K. F.,Cohen, R. J.,Heinig, M. J.,Dewey, K. G. Educational intervention to modify bottle-feeding behaviors among formula- } \\
\text { feeding mothers in the WIC program: impact on infant formula intake and weight gain. J Nutr Educ Behav.2008;40:244-50. }\end{array}$ & IV \\
\hline 305 & $\begin{array}{l}\text { Kavle, J. A.,Flax, V. L.,Abdelmegeid, A.,Salah, F.,Hafez, S.,Ramzy, M.,Hamed, D.,Saleh, G.,Galloway, R. Factors associated with } \\
\text { early growth in Egyptian infants: implications for addressing the dual burden of malnutrition. Matern Child Nutr.2016;12:139-51. }\end{array}$ & Country \\
\hline 306 & $\begin{array}{l}\text { Kersting, M.,Alexy, U.,Sichert-Hellert, W.,Manz, F.,Schoch, G. Measured consumption of commercial infant food products in German } \\
\text { infants: results from the DONALD study. Dortmund Nutritional and Anthropometrical Longitudinally Designed. J Pediatr } \\
\text { Gastroenterol Nutr.1998;27:547-52. }\end{array}$ & DV \\
\hline 307 & $\begin{array}{l}\text { Kesaree, N.,Shivamurty, K. S.,Prakash, B. S., Ramachandra,,Haridas, C. K. Feeding pattern of infants in Devangere. Indian J } \\
\text { Pediatr.1981;48:281-4. }\end{array}$ & Design, IV \\
\hline 308 & Khaldi, F.,Bouguerra, F. Feeding practices, growth, and morbidity in Tunisia. Pediatrics.2000;106:1275-6. & Design, \\
\hline
\end{tabular}




\begin{tabular}{|c|c|c|}
\hline & & Date \\
\hline 309 & $\begin{array}{l}\text { Khan, A. I.,Kabir, I.,Hawkesworth, S.,Ekström, E. C.,Arifeen, S.,Frongillo, E. A.,Persson, L. A. Early invitation to food and/or multiple } \\
\text { micronutrient supplementation in pregnancy does not affect body composition in offspring at } 54 \text { months: Follow-up of the MINIMat } \\
\text { randomised trial, Bangladesh. Maternal and Child Nutrition.2015;11:385-397. }\end{array}$ & IV, Age \\
\hline 310 & Khan, M. U. Breastfeeding, growth and diarrhoea in rural Bangladesh children. Hum Nutr Clin Nutr.1984;38:113-9. & IV \\
\hline 311 & Khan, M. Infant feeding practices in rural Meheran, Comilla, Bangladesh. Am J Clin Nutr.1980;33:2356-64. & IV, DV \\
\hline 312 & $\begin{array}{l}\text { Kholdi N,Zayeri F,Bagheban AA,Khodakarim S,Ramezankhani A. A study of growth failure and its related factors in children from } 0 \\
\text { to } 2 \text { years in Tehran, Iran. Turk J Pediatr.2012;54:38-44. }\end{array}$ & Design \\
\hline 313 & $\begin{array}{l}\text { Kiefte-de Jong, J. C.,de Vries, J. H.,Escher, J. C.,Jaddoe, V. W.,Hofman, A., Raat, H.,Moll, H. A. Role of dietary patterns, sedentary } \\
\text { behaviour and overweight on the longitudinal development of childhood constipation: the Generation R study. Matern Child } \\
\text { Nutr.2013;9:511-23. }\end{array}$ & DV \\
\hline 314 & $\begin{array}{l}\text { Kilaru, A.,Griffiths, P. L.,Ganapathy, S.,Ghosh, S. Community-based nutrition education for improving infant growth in rural } \\
\text { Karnataka. Indian Pediatr.2005;42:425-32. }\end{array}$ & IV \\
\hline 315 & $\begin{array}{l}\text { King,S.,Prawitz,A. D.,Umoren,J.,O'Gorman,T. The impact of high diastase malted barley flour on weight and height of malnourished } \\
\text { children in Panama. Journal of Hunger and Environmental Nutrition.2007;1:23-35. }\end{array}$ & $\begin{array}{l}\text { Health } \\
\text { status, } \\
\text { Age }\end{array}$ \\
\hline 316 & $\begin{array}{l}\text { Klag, E. A.,McNamara, K.,Geraghty, S. R.,Keim, S. A. Associations Between Breast Milk Feeding, Introduction of Solid Foods, and } \\
\text { Weight Gain in the First } 12 \text { Months of Life. Clin Pediatr (Phila).2015;54:1059-67. }\end{array}$ & Design \\
\hline 317 & Kleinman, R. E. Complementary feeding and neuromuscular development. Pediatrics.2000;106:1279. & Design \\
\hline 318 & $\begin{array}{l}\text { Kolacek, S.,Kapetanovic, T.,Zimolo, A.,Luzar, V. Early determinants of cardiovascular risk factors in adults. A. Plasma lipids. Acta } \\
\text { Paediatr.1993;82:699-704. }\end{array}$ & DV \\
\hline 319 & $\begin{array}{l}\text { Koletzko, B.,Dokoupil, K.,Reitmayr, S.,Weimert-Harendza, B.,Keller, E. Dietary fat intakes in infants and primary school children in } \\
\text { Germany. Am J Clin Nutr.2000;72:1392s-1398s. }\end{array}$ & IV, DV \\
\hline 320 & $\begin{array}{l}\text { Koletzko, B.,von Kries, R.,Closa, R.,Escribano, J.,Scaglioni, S.,Giovannini, M.,Beyer, J.,Demmelmair, H.,Anton, B.,Gruszfeld, } \\
\text { D.,Dobrzanska, A.,Sengier, A.,Langhendries, J. P.,Rolland Cachera, M. F.,Grote, V. Can infant feeding choices modulate later } \\
\text { obesity risk?. Am J Clin Nutr.2009;89:1502s-1508s. }\end{array}$ & Design, IV \\
\hline 321 & $\begin{array}{l}\text { Koletzko, B.,von Kries, R.,Closa, R.,Escribano, J.,Scaglioni, S.,Giovannini, M.,Beyer, J.,Demmelmair, H.,Gruszfeld, D.,Dobrzanska, } \\
\text { A.,Sengier, A.,Langhendries, J. P.,Rolland Cachera, M. F.,Grote, V. Lower protein in infant formula is associated with lower weight } \\
\text { up to age } 2 \text { y: a randomized clinical trial. Am J Clin Nutr.2009;89:1836-45. }\end{array}$ & IV \\
\hline 322 & Koletzko, B. Complementary foods and the development of food allergy. Pediatrics.2000;106:1285. & Design \\
\hline 323 & $\begin{array}{l}\text { Konstantynowicz, J.,Nguyen, T. V.,Kaczmarski, M.,Jamiolkowski, J.,Piotrowska-Jastrzebska, J.,Seeman, E. Fractures during } \\
\text { growth: potential role of a milk-free diet. Osteoporos Int.2007;18:1601-7. }\end{array}$ & Age \\
\hline 324 & $\begin{array}{l}\text { Kramer, M. S.,Fombonne, E.,Matush, L.,Bogdanovich, N.,Dahhou, M.,Platt, R. W. Long-term behavioural consequences of infant } \\
\text { feeding: the limits of observational studies. Paediatr Perinat Epidemiol.2011;25:500-6. }\end{array}$ & IV \\
\hline
\end{tabular}




\begin{tabular}{|c|c|c|}
\hline 325 & $\begin{array}{l}\text { Kramer, M. S.,Guo, T.,Platt, R. W.,Shapiro, S.,Collet, J. P.,Chalmers, B.,Hodnett, E.,Sevkovskaya, Z., Dzikovich, I.,Vanilovich, I. } \\
\text { Breastfeeding and infant growth: biology or bias?. Pediatrics.2002;110:343-7. }\end{array}$ & IV \\
\hline 326 & $\begin{array}{l}\text { Kramer, M. S.,Moodie, E. E.,Dahhou, M.,Platt, R. W. Breastfeeding and infant size: evidence of reverse causality. Am J } \\
\text { Epidemiol.2011;173:978-83. }\end{array}$ & IV, DV \\
\hline 327 & $\begin{array}{l}\text { Kramer, M. S.,Moodie, E. E.,Platt, R. W. Infant feeding and growth: can we answer the causal question?. } \\
\text { Epidemiology.2012;23:790-4. }\end{array}$ & Design \\
\hline 328 & $\begin{array}{l}\text { Kramer, M. S. Do breast-feeding and delayed introduction of solid foods protect against subsequent obesity?. J } \\
\text { Pediatr.1981;98:883-7. }\end{array}$ & Design \\
\hline 329 & $\begin{array}{l}\text { Krebs,N. F. Culbertson D. L. Westcott J. L. Sherlock L. Hambidge K. M. Normal Iron Status in Breastfed Infants Consuming Meat as } \\
\text { an Early Complementary Food. Pediatric Academic Societies Annual Meeting.2011. }\end{array}$ & Design \\
\hline 330 & $\begin{array}{l}\text { Krebs,N. F.,Hambidge,K. M.,Westcott,J. E.,Miller,L. V.,Sian,L.,Bell,M.,Grunwald,G. Exchangeable zinc pool size in infants is related } \\
\text { to key variables of zinc homeostasis. J Nutr.2003;133:1498s-501s. }\end{array}$ & DV \\
\hline 331 & $\begin{array}{l}\text { Krebs,N. F.,Mazariegos,M.,Chomba,E.,Sami,N.,Pasha,O.,Tshefu,A.,Carlo,W. A.,Goldenberg,R. L.,Bose,C. L.,Wright,L. L.,Koso- } \\
\text { Thomas,M.,Goco,N.,Kindem,M.,McClure,E. M.,Westcott,J.,Garces,A.,Lokangaka,A.,Manasyan,A.,Imenda,E.,Hartwell,T. } \\
\text { D.,Hambidge,K. M. Randomized controlled trial of meat compared with multimicronutrient-fortified cereal in infants and toddlers with } \\
\text { high stunting rates in diverse settings. Am J Clin Nutr.2012;96:840-7. }\end{array}$ & Country \\
\hline 332 & $\begin{array}{l}\text { Krebs,N. F.,Westcott,J. E.,Culbertson,D. L.,Sian,L.,Miller,L. V.,Hambidge,K. M. Comparison of complementary feeding strategies to } \\
\text { meet zinc requirements of older breastfed infants. Am J Clin Nutr.2012;96:30-5. }\end{array}$ & DV \\
\hline 333 & $\begin{array}{l}\text { Kronborg, H.,Foverskov, E.,Vaeth, M. Predictors for early introduction of solid food among Danish mothers and infants: an } \\
\text { observational study. BMC Pediatr.2014;14:243. }\end{array}$ & $\begin{array}{l}\text { Design, } \\
\text { DV }\end{array}$ \\
\hline 334 & $\begin{array}{l}\text { Kuklina, E. V.,Ramakrishnan, U.,Stein, A. D.,Barnhart, H. H.,Martorell, R. Growth and diet quality are associated with the attainment } \\
\text { of walking in rural Guatemalan infants. J Nutr.2004;134:3296-300. }\end{array}$ & IV \\
\hline 335 & $\begin{array}{l}\text { Kumar, V.,Sharma, S.,Khanna, P.,Vanaja, K. Breast vs bottle feeding-impact on growth in urban infants. Indian J } \\
\text { Pediatr.1981;48:271-5. }\end{array}$ & IV, DV \\
\hline 336 & $\begin{array}{l}\text { Kusin, J. A., Kardjati, S.,van Steenbergen, W. M.,Renqvist, U. H. Nutritional transition during infancy in East Java, Indonesia: } 2 \text {. A } \\
\text { longitudinal study of growth in relation to the intake of breast milk and additional foods. Eur J Clin Nutr.1991;45:77-84. }\end{array}$ & IV \\
\hline 337 & Kusin, J. A.,Kardjati, S.,van Steenbergen, W. Traditional infant feeding practices: right or wrong?. Soc Sci Med.1985;21:283-6. & $\begin{array}{l}\text { IV, } \\
\text { Country }\end{array}$ \\
\hline 338 & $\begin{array}{l}\text { Kwok, M. K.,Leung, G. M.,Lam, T. H.,Schooling, C. M. Breastfeeding, childhood milk consumption, and onset of puberty. } \\
\text { Pediatrics.2012;130:e631-9. }\end{array}$ & DV \\
\hline 339 & $\begin{array}{l}\text { Laitinen,K.,Kalliomaki,M.,Poussa,T.,Lagstrom,H.,Isolauri,E. Evaluation of diet and growth in children with and without atopic } \\
\text { eczema: follow-up study from birth to } 4 \text { years. Br J Nutr.2005;94:565-74. }\end{array}$ & IV, DV \\
\hline 340 & $\begin{array}{l}\text { Lampe,J. B.,Velez,N. The effect of prolonged bottle feeding on cow's milk intake and iron stores at } 18 \text { months of age. Clin Pediatr } \\
\text { (Phila).1997;36:569-72. }\end{array}$ & Design \\
\hline 341 & Lang B,Moynihan P. The weaning diet: promoting growth while safeguarding dental health. Quintessence Int.2006;37:632-6. & Design \\
\hline 342 & $\begin{array}{l}\text { Larnkjaer, A.,Hoppe, C.,Molgaard, C.,Michaelsen, K. F. The effects of whole milk and infant formula on growth and IGF-I in late } \\
\text { infancy. Eur J Clin Nutr.2009;63:956-63. }\end{array}$ & IV \\
\hline
\end{tabular}




\begin{tabular}{|c|c|c|}
\hline 343 & $\begin{array}{l}\text { Lasky, P. A.,Eichelberger, K. M. Implications, considerations, and nursing interventions of obesity in neonatal and preschool } \\
\text { patients. Nurs Clin North Am.1982;17:199-205. }\end{array}$ & Design \\
\hline 344 & $\begin{array}{l}\text { Lauzon-Guillain, Bd,Wijndaele, K.,Clark, M.,Acerini, C. L.,Hughes, I. A., Dunger, D. B.,Wells, J. C., Ong, K. K. Breastfeeding and } \\
\text { infant temperament at age three months. PLoS One.2012;7:e29326. }\end{array}$ & Design, IV \\
\hline 345 & $\begin{array}{l}\text { Lawlor, D. A., Riddoch, C. J.,Page, A. S.,Andersen, L. B.,Wedderkopp, N.,Harro, M.,Stansbie, D.,Smith, G. D. Infant feeding and } \\
\text { components of the metabolic syndrome: findings from the European Youth Heart Study. Arch Dis Child.2005;90:582-8. }\end{array}$ & IV \\
\hline 346 & Lee, K. Crying and behavior pattern in breast- and formula-fed infants. Early Hum Dev.2000;58:133-40. & $\begin{array}{l}\text { Design, IV, } \\
\text { DV }\end{array}$ \\
\hline 347 & $\begin{array}{l}\text { Leermakers, E. T.,Kiefte-de Jong, J. C.,Hofman, A.,Jaddoe, V. W.,Franco, O. H. Lutein intake at the age of } 1 \text { year and } \\
\text { cardiometabolic health at the age of } 6 \text { years: the Generation R Study. Br J Nutr.2015;114:970-8. }\end{array}$ & IV \\
\hline 348 & Legovic, M.,Ostric, L. The effects of feeding methods on the growth of the jaws in infants. ASDC J Dent Child.1991;58:253-5. & $\begin{array}{l}\text { Design, IV, } \\
\text { DV }\end{array}$ \\
\hline 349 & $\begin{array}{l}\text { Lei, D. L.,Chaves, S. P.,Paes, A. T.,Escuder, M. M.,Ribeiro, A. B.,Freire, R. D.,Lerner, B. R. Risk of linear growth retardation during } \\
\text { the first two years of life: a new approach. Eur J Clin Nutr.1999;53:456-60. }\end{array}$ & IV \\
\hline 350 & $\begin{array}{l}\text { Leonard, W. R.,Dewalt, K. M.,Stansbury, J. P.,McCaston, M. K. Influence of dietary quality on the growth of highland and coastal } \\
\text { Ecuadorian children. Am J Hum Biol.2000;12:825-837. }\end{array}$ & IV \\
\hline 351 & $\begin{array}{l}\text { Leroy, J. L.,Ruel, M.,Habicht, J. P.,Frongillo, E. A. Using height-for-age differences (HAD) instead of height-for-age z-scores (HAZ) } \\
\text { for the meaningful measurement of population-level catch-up in linear growth in children less than } 5 \text { years of age. BMC } \\
\text { Pediatr.2015;15:145. }\end{array}$ & IV \\
\hline 352 & $\begin{array}{l}\text { Leung, S. S.,Peng, C. X.,Xu, Y. Y.,Liu, K. M.,Quan, X. J.,Lui, S.,Davies, D. P. Comparative study of growth of Chinese infants: Hong } \\
\text { Kong versus Guangzhou. J Trop Pediatr.1994;40:166-71. }\end{array}$ & IV, DV \\
\hline 353 & Leung,S.,Davies,D. P. Infant feeding and growth of Chinese infants: birth to 2 years. Paediatr Perinat Epidemiol.1994;8:301-13. & IV, DV \\
\hline 354 & $\begin{array}{l}\text { Leventakou, V.,Roumeliotaki, T.,Koutra, K.,Vassilaki, M.,Mantzouranis, E.,Bitsios, P.,Kogevinas, M.,Chatzi, L. Breastfeeding } \\
\text { duration and cognitive, language and motor development at } 18 \text { months of age: Rhea mother-child cohort in Crete, Greece. J } \\
\text { Epidemiol Community Health.2015;69:232-9. }\end{array}$ & IV \\
\hline 355 & $\begin{array}{l}\text { Li, C., Kaur, H.,Choi, W. S.,Huang, T. T.,Lee, R. E.,Ahluwalia, J. S. Additive interactions of maternal prepregnancy BMI and breast- } \\
\text { feeding on childhood overweight. Obes Res.2005;13:362-71. }\end{array}$ & IV \\
\hline 356 & $\begin{array}{l}\text { Li, R.,Magadia, J.,Fein, S. B.,Grummer-Strawn, L. M. Risk of bottle-feeding for rapid weight gain during the first year of life. Arch } \\
\text { Pediatr Adolesc Med.2012;166:431-6. }\end{array}$ & IV \\
\hline 357 & $\begin{array}{l}\text { Lin, S. L.,Leung, G. M.,Lam, T. H.,Schooling, C. M. Timing of solid food introduction and obesity: Hong Kong's "children of 1997" } \\
\text { birth cohort. Pediatrics.2013;131:e1459-67. }\end{array}$ & Design \\
\hline 358 & $\begin{array}{l}\text { Lind,T.,Lonnerdal,B.,Persson,L. A.,Stenlund,H.,Tennefors,C.,Hernell,O. Effects of weaning cereals with different phytate contents on } \\
\text { hemoglobin, iron stores, and serum zinc: a randomized intervention in infants from } 6 \text { to } 12 \text { mo of age. Am J Clin Nutr.2003;78:168- } \\
75 .\end{array}$ & DV \\
\hline 359 & Livingstone V. Failure to thrive while breastfeeding. Breastfeed Med.2006;1:108-11. & Design \\
\hline
\end{tabular}




\begin{tabular}{|c|c|c|}
\hline 360 & $\begin{array}{l}\text { Lozoff, B.,De Andraca, I.,Castillo, M.,Smith, J. B.,Walter, T.,Pino, P. Behavioral and developmental effects of preventing iron- } \\
\text { deficiency anemia in healthy full-term infants. Pediatrics.2003;112:846-54. }\end{array}$ & IV \\
\hline 361 & $\begin{array}{l}\text { Lucas, R.,Judge, M.,Sajdlowska, J.,Cong, X.,McGrath, J. M.,Brandon, D. Effect of Maternal Body Mass Index on Infant } \\
\text { Breastfeeding Behaviors and Exclusive Direct Breastfeeding. J Obstet Gynecol Neonatal Nurs.2015;44:772-83. }\end{array}$ & DV \\
\hline 362 & $\begin{array}{l}\text { Lutter, C. K.,Mora, J. O.,Habicht, J. P.,Rasmussen, K. M.,Robson, D. S.,Herrera, M. G. Age-specific responsiveness of weight and } \\
\text { length to nutritional supplementation. Am J Clin Nutr.1990;51:359-64. }\end{array}$ & IV \\
\hline 363 & $\begin{array}{l}\text { Lutter, C. K.,Mora, J. O.,Habicht, J. P.,Rasmussen, K. M.,Robson, D. S.,Sellers, S. G.,Super, C. M.,Herrera, M. G. Nutritional } \\
\text { supplementation: effects on child stunting because of diarrhea. Am J Clin Nutr.1989;50:1-8. }\end{array}$ & IV \\
\hline 364 & $\begin{array}{l}\text { Lutter,C. K.,Rodriguez,A.,Fuenmayor,G.,Avila,L.,Sempertegui,F.,Escobar,J. Growth and micronutrient status in children receiving a } \\
\text { fortified complementary food. J Nutr.2008;138:379-88. }\end{array}$ & IV \\
\hline 365 & $\begin{array}{l}\text { Luukkainen,P.,Salo,M. K.,Visakorpi,J. K.,Raiha,N. C.,Nikkari,T. Impact of solid food on plasma arachidonic and docosahexaenoic } \\
\text { acid status of term infants at } 8 \text { months of age. J Pediatr Gastroenterol Nutr.1996;23:229-34. }\end{array}$ & IV, DV \\
\hline 366 & MacCarthy, D. Psychological influences affecting growth. Health Visit.1980;53:470-6. & Design \\
\hline 367 & $\begin{array}{l}\text { Magarey, A.,Kavian, F.,Scott, J. A.,Markow, K.,Daniels, L. Feeding Mode of Australian Infants in the First } 12 \text { Months of Life: An } \\
\text { Assessment against National Breastfeeding Indicators. J Hum Lact.2015. }\end{array}$ & DV \\
\hline 368 & $\begin{array}{l}\text { Maimaitiming, T.,Wang, Y. P. Feeding and intellectual development in infants and children from Uygur, Kazak, and Han nationality: } \\
\text { A comparison of } 454 \text { cases. Journal of Clinical Rehabilitative Tissue Engineering Research.2007;11:10710-10712. }\end{array}$ & Design \\
\hline 369 & $\begin{array}{l}\text { Makrides,M.,Gould,J. F.,Gawlik,N. R.,Yelland,L. N.,Smithers,L. G.,Anderson,P. J.,Gibson,R. A. Four-year follow-up of children born } \\
\text { to women in a randomized trial of prenatal DHA supplementation. Jama.2014;311:1802-4. }\end{array}$ & IV \\
\hline 370 & $\begin{array}{l}\text { Male,C.,Persson,L. A.,Freeman,V.,Guerra,A.,van't Hof,M. A.,Haschke,F. Prevalence of iron deficiency in 12-mo-old infants from } 11 \\
\text { European areas and influence of dietary factors on iron status (Euro-Growth study). Acta Paediatr.2001;90:492-8. }\end{array}$ & DV \\
\hline 371 & $\begin{array}{l}\text { Mallan, K. M.,Fildes, A.,Magarey, A. M.,Daniels, L. A. The Relationship between Number of Fruits, Vegetables, and Noncore Foods } \\
\text { Tried at Age } 14 \text { Months and Food Preferences, Dietary Intake Patterns, Fussy Eating Behavior, and Weight Status at Age } 3.7 \text { Years. } \\
\text { J Acad Nutr Diet.2016;116:630-7. }\end{array}$ & DV \\
\hline 372 & $\begin{array}{l}\text { Mallard,S. R.,Houghton,L. A.,Filteau,S.,Mullen,A.,Nieuwelink,J.,Chisenga,M.,Siame,J.,Gibson,R. S. Dietary diversity at } 6 \text { months of } \\
\text { age is associated with subsequent growth and mediates the effect of maternal education on infant growth in urban Zambia. J } \\
\text { Nutr.2014;144:1818-25. }\end{array}$ & Country \\
\hline 373 & $\begin{array}{l}\text { Mamabolo, R. L.,Alberts, M.,Mbenyane, G. X.,Steyn, N. P.,Nthangeni, N. G.,Delemarre-Van De Waal, H. A.,Levitt, N. S. Feeding } \\
\text { practices and growth of infants from birth to } 12 \text { months in the central region of the Limpopo Province of South Africa. } \\
\text { Nutrition.2004;20:327-33. }\end{array}$ & IV, DV \\
\hline 374 & $\begin{array}{l}\text { Mamabolo,R. L.,Alberts,M.,Levitt,N. S.,Delemarre-van de Waal,H. A.,Steyn,N. P. Association between insulin-like growth factor-1, } \\
\text { insulin-like growth factor-binding protein-1 and leptin levels with nutritional status in 1-3-year-old children, residing in the central } \\
\text { region of Limpopo Province, South Africa. Br J Nutr.2007;98:762-9. }\end{array}$ & IV \\
\hline 375 & $\begin{array}{l}\text { Mamun, A. A.,Lawlor, D. A.,O'Callaghan, M. J.,Williams, G. M.,Najman, J. M. Family and early life factors associated with changes } \\
\text { in overweight status between ages } 5 \text { and } 14 \text { years: findings from the Mater University Study of Pregnancy and its outcomes. Int J } \\
\text { Obes (Lond).2005;29:475-82. }\end{array}$ & IV \\
\hline
\end{tabular}




\begin{tabular}{|c|c|c|}
\hline 376 & $\begin{array}{l}\text { Manjrekar, C., Vishalakshi, M. P.,Begum, N. J.,Padma, G. N. Breast feeding ability of undernourished mothers and physical } \\
\text { development of their infants during 0-1 year. Indian Pediatr.1985;22:801-9. }\end{array}$ & IV, DV \\
\hline 377 & $\begin{array}{l}\text { Marques,R. C.,Dorea,J. G.,Bernardi,J. V.,Bastos,W. R.,Malm,O. Maternal fish consumption in the nutrition transition of the Amazon } \\
\text { Basin: growth of exclusively breastfed infants during the first } 5 \text { years. Ann Hum Biol.2008;35:363-77. }\end{array}$ & IV \\
\hline 378 & $\begin{array}{l}\text { Marquis, G. S.,Habicht, J. P.,Lanata, C. F.,Black, R. E.,Rasmussen, K. M. Association of breastfeeding and stunting in Peruvian } \\
\text { toddlers: an example of reverse causality. Int J Epidemiol.1997;26:349-56. }\end{array}$ & Design \\
\hline 379 & $\begin{array}{l}\text { Marquis, G. S.,Habicht, J. P.,Lanata, C. F.,Black, R. E.,Rasmussen, K. M. Breast milk or animal-product foods improve linear growth } \\
\text { of Peruvian toddlers consuming marginal diets. Am J Clin Nutr.1997;66:1102-9. }\end{array}$ & Design \\
\hline 380 & $\begin{array}{l}\text { Martin, R. M.,McCarthy, A.,Smith, G. D.,Davies, D. P.,Ben-Shlomo, Y. Infant nutrition and blood pressure in early adulthood: the } \\
\text { Barry Caerphilly Growth study. Am J Clin Nutr.2003;77:1489-97. }\end{array}$ & IV, DV \\
\hline 381 & $\begin{array}{l}\text { Martin, R. M.,Smith, G. D.,Mangtani, P.,Frankel, S.,Gunnell, D. Association between breast feeding and growth: the Boyd-Orr cohort } \\
\text { study. Arch Dis Child Fetal Neonatal Ed.2002;87:F193-201. }\end{array}$ & IV \\
\hline 382 & $\begin{array}{l}\text { Martines, J. C.,Habicht, J. P.,Ashworth, A.,Kirkwood, B. R. Weaning in southern Brazil: is there a "weanling's dilemma"?. J } \\
\text { Nutr.1994;124:1189-98. }\end{array}$ & IV \\
\hline 383 & $\begin{array}{l}\text { Matsuzaki,M.,Kuper,H.,Kulkarni,B.,Radhakrishna,K. V.,Viljakainen,H.,Taylor,A. E.,Sullivan,R.,Bowen,L.,Tobias,J. H.,Ploubidis,G. } \\
\text { B.,Wells,J. C.,Prabhakaran,D.,Smith,G. D.,Ebrahim,S.,Ben-Shlomo,Y.,Kinra,S. Life-course determinants of bone mass in young } \\
\text { adults from a transitional rural community in India: The Andhra Pradesh Children and Parents Study (APCAPS). American Journal } \\
\text { of Clinical Nutrition.2014;99:1450-1459. }\end{array}$ & $\begin{array}{l}\text { Design, IV, } \\
\text { Age }\end{array}$ \\
\hline 384 & $\begin{array}{l}\text { May, A. L.,Dietz, W. H. The Feeding Infants and Toddlers Study 2008: opportunities to assess parental, cultural, and environmental } \\
\text { influences on dietary behaviors and obesity prevention among young children. J Am Diet Assoc.2010;110:S11-5. }\end{array}$ & Design \\
\hline 385 & $\begin{array}{l}\text { Mazariegos,M.,Hambidge,K. M.,Westcott,J. E.,Solomons,N. W.,Raboy,V.,Das,A.,Goco,N.,Kindem,M.,Wright,L. L.,Krebs,N. F. } \\
\text { Neither a zinc supplement nor phytate-reduced maize nor their combination enhance growth of 6- to 12-month-old Guatemalan } \\
\text { infants. J Nutr.2010;140:1041-8. }\end{array}$ & Country \\
\hline 386 & McGuire, E. Breastfeeding and high maternal body mass index. Breastfeed Rev.2013;21:7-14. & Design \\
\hline 387 & $\begin{array}{l}\text { McNeill, G. Birth weight, feeding practices and weight-for-age of Punjabi children in the UK and in the rural Punjab. Hum Nutr Clin } \\
\text { Nutr.1985;39:69-72. }\end{array}$ & Design \\
\hline 388 & $\begin{array}{l}\text { Mehta KC,Specker BL,Bartholmey S,Giddens J,Ho ML. Trial on timing of introduction to solids and food type on infant growth. } \\
\text { Pediatrics.1998;102:569-73. }\end{array}$ & IV \\
\hline 389 & $\begin{array}{l}\text { Mei, H.,Guo, B.,Yin, B.,Liang, X.,Adair, L.,Thompson, A.,Zhang, J. Interactive Effects of Early Exclusive Breastfeeding and Pre- } \\
\text { Pregnancy Maternal Weight Status on Young Children's BMI - A Chinese Birth Cohort. PLoS One.2015;10:e0144357. }\end{array}$ & IV \\
\hline 390 & $\begin{array}{l}\text { Meldrum,S. J.,D'Vaz,N.,Simmer,K.,Dunstan,J. A.,Hird,K.,Prescott,S. L. Effects of high-dose fish oil supplementation during early } \\
\text { infancy on neurodevelopment and language: a randomised controlled trial. Br J Nutr.2012;108:1443-54. }\end{array}$ & IV \\
\hline 391 & $\begin{array}{l}\text { Melnik, B. Growth and puberty in German children: is there still a positive secular trend? Consumption of milk as a vital factor in } \\
\text { growth development..Bettina Gohlke, Woelfle JF. volume 23/2009. Deutsches Aerzteblatt International.2009;106:656-656. }\end{array}$ & Design \\
\hline 392 & $\begin{array}{l}\text { Mennella, J. A.,Castor, S. M. Sensitive period in flavor learning: effects of duration of exposure to formula flavors on food likes } \\
\text { during infancy. Clin Nutr.2012;31:1022-5. }\end{array}$ & DV \\
\hline
\end{tabular}




\begin{tabular}{|c|c|c|}
\hline 393 & $\begin{array}{l}\text { Merten, S.,Dratva, J.,Ackermann-Liebrich, U. Do baby-friendly hospitals influence breastfeeding duration on a national level?. } \\
\text { Pediatrics.2005;116:e702-8. }\end{array}$ & Design, IV \\
\hline 394 & $\begin{array}{l}\text { Michaelsen, K. F.,Johansen, J. S., Samuelson, G.,Price, P. A., Christiansen, C. Serum bone gamma-carboxyglutamic acid protein in } \\
\text { a longitudinal study of infants: lower values in formula-fed infants. Pediatr Res.1992;31:401-5. }\end{array}$ & IV \\
\hline 395 & Michaelsen, K. F. Are there negative effects of an excessive protein intake?. Pediatrics.2000;106:1293. & Design \\
\hline 396 & $\begin{array}{l}\text { Michaelsen,K. F.,Milman,N.,Samuelson,G. A longitudinal study of iron status in healthy Danish infants: effects of early iron status, } \\
\text { growth velocity and dietary factors. Acta Paediatr.1995;84:1035-44. }\end{array}$ & IV, DV \\
\hline 397 & Michaelsen,K. F. Cows' milk in complementary feeding. Pediatrics.2000;106:1302-3. & Design \\
\hline 398 & Michaelsen,K. F. Nutrition and growth during infancy. The Copenhagen Cohort Study. Acta Paediatr Suppl.1997;420:1-36. & Design, IV \\
\hline 399 & $\begin{array}{l}\text { Michels, K. B.,Willett, W. C.,Graubard, B. I.,Vaidya, R. L.,Cantwell, M. M.,Sansbury, L. B.,Forman, M. R. A longitudinal study of } \\
\text { infant feeding and obesity throughout life course. Int J Obes (Lond).2007;31:1078-85. }\end{array}$ & IV \\
\hline 400 & $\begin{array}{l}\text { Mihrshahi, S.,Battistutta, D.,Magarey, A.,Daniels, L. A. Determinants of rapid weight gain during infancy: baseline results from the } \\
\text { NOURISH randomised controlled trial. BMC Pediatr.2011;11:99. }\end{array}$ & Design \\
\hline 401 & $\begin{array}{l}\text { Millar, L.,Kremer, P.,de Silva-Sanigorski, A.,McCabe, M. P.,Mavoa, H.,Moodie, M.,Utter, J.,Bell, C.,Malakellis, M.,Mathews, } \\
\text { L.,Roberts, G.,Robertson, N.,Swinburn, B. A. Reduction in overweight and obesity from a 3-year community-based intervention in } \\
\text { Australia: the 'It's Your Move!' project. Obes Rev.2011;12 Suppl 2:20-8. }\end{array}$ & IV, Age \\
\hline 402 & $\begin{array}{l}\text { Mimouni-Bloch, A.,Kachevanskaya, A.,Mimouni, F. B.,Shuper, A., Raveh, E.,Linder, N. Breastfeeding may protect from developing } \\
\text { attention-deficit/hyperactivity disorder. Breastfeed Med.2013;8:363-7. }\end{array}$ & IV \\
\hline 403 & Mittal, S.,Gupta, M. C. Evaluation of a supplementary feeding programme through take home system. J Trop Pediatr.1980;26:50-3. & IV \\
\hline 404 & $\begin{array}{l}\text { Miura T,Fukuda M,Mizuno M,Ohte N. Need for public awareness regarding low birth weight and bottle feeding. Hypertens } \\
\text { Res.2016. }\end{array}$ & Design \\
\hline 405 & $\begin{array}{l}\text { Molgaard, C.,Larnkjaer, A.,Mark, A. B.,Michaelsen, K. F. Are early growth and nutrition related to bone health in adolescence? The } \\
\text { Copenhagen Cohort Study of infant nutrition and growth. Am J Clin Nutr.2011;94:1865s-1869s. }\end{array}$ & IV \\
\hline 406 & $\begin{array}{l}\text { Moller, L. M.,de Hoog, M. L.,van Eijsden, M.,Gemke, R. J.,Vrijkotte, T. G. Infant nutrition in relation to eating behaviour and fruit and } \\
\text { vegetable intake at age } 5 \text { years. Br J Nutr.2013;109:564-71. }\end{array}$ & DV \\
\hline 407 & $\begin{array}{l}\text { Monterrosa EC,Frongillo EA,Neufeld LM,Egan KA,Ramakrishnan U,Rasmussen KM. Maternal pre-pregnancy body mass index is } \\
\text { not associated with infant and young child feeding in low-income Mexican children 1-24 months old. Matern Child Nutr.2015;11:215- } \\
28 .\end{array}$ & DV \\
\hline 408 & $\begin{array}{l}\text { Monterrosa,E. C.,Frongillo,E. A.,Vasquez-Garibay,E. M.,Romero-Velarde,E.,Casey,L. M.,Willows,N. D. Predominant breast-feeding } \\
\text { from birth to six months is associated with fewer gastrointestinal infections and increased risk for iron deficiency among infants. J J } \\
\text { Nutr.2008;138:1499-504. }\end{array}$ & IV \\
\hline 409 & $\begin{array}{l}\text { Monti G,Viola S,Baro C,Cresi F,Tovo PA,Moro G,Ferrero MP,Conti A,Bertino E. Tolerability of donkey's milk in } 92 \text { highly-problematic } \\
\text { cow's milk allergic children. J Biol Regul Homeost Agents.2012;26:75-82. }\end{array}$ & IV \\
\hline 410 & Moore,S. E.,Prentice,A. M.,Wagatsuma,Y.,Fulford,A. J.,Collinson,A. C.,Raqib,R.,Vahter,M.,Persson,L. A.,Arifeen,S. E. Early-life & IV, DV \\
\hline
\end{tabular}




\begin{tabular}{|c|c|c|}
\hline & nutritional and environmental determinants of thymic size in infants born in rural Bangladesh. Acta Paediatr.2009;98:1168-75. & \\
\hline 411 & Moreno M. Early infant feeding and obesity risk. JAMA Pediatr.2014;168:1084. & Design \\
\hline 412 & Morgan JB,Mumford PM. A follow-up study of nutrition and anthropometry in pre-school children. Proc Nutr Soc.1980;39:5a. & Design \\
\hline 413 & $\begin{array}{l}\text { Morgan, J. B.,Lucas, A.,Fewtrell, M. S. Does weaning influence growth and health up to } 18 \text { months?. Archives of Disease in } \\
\text { Childhood: Education and Practice Edition.2004;89:728-733. }\end{array}$ & Design \\
\hline 414 & $\begin{array}{l}\text { Morley,R.,Abbott,R.,Fairweather-Tait,S.,MacFadyen,U.,Stephenson,T.,Lucas,A. Iron fortified follow on formula from } 9 \text { to } 18 \text { months } \\
\text { improves iron status but not development or growth: a randomised trial. Arch Dis Child.1999;81:247-52. }\end{array}$ & IV \\
\hline 415 & $\begin{array}{l}\text { Morrow-Tlucak, M.,Haude, R. H.,Ernhart, C. B. Breastfeeding and cognitive development in the first } 2 \text { years of life. Soc Sci } \\
\text { Med.1988;26:635-9. }\end{array}$ & IV \\
\hline 416 & Morton,R. E.,Nysenbaum,A.,Price,K. Iron status in the first year of life. J Pediatr Gastroenterol Nutr.1988;7:707-12. & DV \\
\hline 417 & $\begin{array}{l}\text { Mumford, P.,Morgan, J. B. A longitudinal study of nutrition and growth of infants initially on the upper and lower centile for weight } \\
\text { and age. Int J Obes.1982;6:335-41. }\end{array}$ & IV, DV \\
\hline 418 & $\begin{array}{l}\text { Musaad, S. M.,Donovan, S. M.,Fiese, B. H. Parental perception of child weight in the first two years-of-life: a potential link between } \\
\text { infant feeding and preschoolers' diet. Appetite.2015;91:90-100. }\end{array}$ & $\begin{array}{l}\text { Design, } \\
\text { DV }\end{array}$ \\
\hline 419 & Nagahara, K.,Dobashi, K.,Itabashi, K. Feeding choice has a gender-associated effect on infant growth. Pediatr Int.2013;55:481-7. & IV \\
\hline 420 & $\begin{array}{l}\text { Nakao, R. M. Effects of an education program on the health and illness profile of rural breast-fed babies. Philipp J Nurs.1988;58:12- } \\
8 .\end{array}$ & IV \\
\hline 421 & $\begin{array}{l}\text { Narese, F.,Puccio, G.,Mazzucco, W.,Falzone, A.,Venturella, V.,Narese, D.,Capra, E. Earlier appearance of the ossification center of } \\
\text { the femoral head in breast-fed versus formula-fed infants. Nutrition.2011;27:1108-11. }\end{array}$ & Design, IV \\
\hline 422 & $\begin{array}{l}\text { Nauta,A. J.,Garssen,J. Nutritional programming of immune defense against infections in early life. Nutricia Research, Utrecht, } \\
\text { Netherlands.2014;12:113-126. }\end{array}$ & Design \\
\hline 423 & $\begin{array}{l}\text { Newman,J. E.,Garces,A.,Mazariegos,M.,Michael Hambidge,K.,Manasyan,A.,Tshefu,A.,Lokangaka,A.,Sami,N.,Carlo,W. A.,Bose,C. } \\
\text { L.,Pasha,O.,Goco,N.,Chomba,E.,Goldenberg,R. L.,Wright,L. L.,Koso-Thomas,M.,Krebs,N. F. Theory-driven process evaluation of a } \\
\text { complementary feeding trial in four countries. Health Educ Res.2014;29:297-305. }\end{array}$ & $\begin{array}{l}\text { Design, } \\
\text { DV }\end{array}$ \\
\hline 424 & $\begin{array}{l}\text { Ng, S. C.,Chong, Y. S.,Rauff, M.,Myo, Z. M.,Nurfarah, C.,Deurenberg, P. R. The influence of breast feeding compared to formula } \\
\text { feeding on infant adiposity. Ann Acad Med Singapore.2004;33:S75. }\end{array}$ & Design, IV \\
\hline 425 & $\begin{array}{l}\text { Nguyen, N. D.,Allen, J. R.,Peat, J. K.,Schofield, W. N.,Nossar, V.,Eisenbruch, M.,Gaskin, K. J. Growth and feeding practices of } \\
\text { Vietnamese infants in Australia. Eur J Clin Nutr.2004;58:356-62. }\end{array}$ & IV, DV \\
\hline 426 & $\begin{array}{l}\text { Nielsen GA,Thomsen BL,Michaelsen KF. Influence of breastfeeding and complementary food on growth between } 5 \text { and } 10 \text { months. } \\
\text { Acta Paediatr.1998;87:911-7. }\end{array}$ & Design \\
\hline 427 & $\begin{array}{l}\text { Niinikoski, H.,Lagstrom, H.,Jokinen, E.,Siltala, M.,Ronnemaa, T.,Viikari, J., Raitakari, O. T.,Jula, A.,Marniemi, J.,Nanto-Salonen, } \\
\text { K.,Simell, O. Impact of repeated dietary counseling between infancy and } 14 \text { years of age on dietary intakes and serum lipids and } \\
\text { lipoproteins: the STRIP study. Circulation.2007;116:1032-40. }\end{array}$ & IV \\
\hline
\end{tabular}




\begin{tabular}{|c|c|c|}
\hline 428 & $\begin{array}{l}\text { Northstone, K.,Emmett, P.,Nethersole, F. The effect of age of introduction to lumpy solids on foods eaten and reported feeding } \\
\text { difficulties at } 6 \text { and } 15 \text { months. J Hum Nutr Diet.2001;14:43-54. }\end{array}$ & DV \\
\hline 429 & $\begin{array}{l}\text { Novotny, R.,Coleman, P.,Tenorio, L.,Davison, N.,Camacho, T.,Ramirez, V.,Vijayadeva, V.,Untalan, P., Tudela, M. D. Breastfeeding } \\
\text { is associated with lower body mass index among children of the Commonwealth of the Northern Mariana Islands. J Am Diet } \\
\text { Assoc.2007;107:1743-6. }\end{array}$ & Design, IV \\
\hline 430 & $\begin{array}{l}\text { Ntouva, A., Rogers, I.,MacAdam, A.,Emmett, P. Weaning practices and iron status of exclusively breast fed infants. Journal of } \\
\text { Human Nutrition \& Dietetics.2011;24:297-298 2p. }\end{array}$ & Design \\
\hline 431 & $\begin{array}{l}\text { Nwaru BI,Takkinen HM,Niemela O,Kaila M,Erkkola M,Ahonen S, Haapala AM,Kenward MG,Pekkanen J,Lahesmaa R, Kere J,Simell } \\
\text { O,Veijola R,llonen J,Hyoty H,Knip M,Virtanen SM. Timing of infant feeding in relation to childhood asthma and allergic diseases. J } \\
\text { Allergy Clin Immunol.2013;131:78-86. }\end{array}$ & DV \\
\hline 432 & Obatolu, V. A. Growth pattern of infants fed with a mixture of extruded malted maize and cowpea. Nutrition.2003;19:174-8. & Country \\
\hline 433 & $\begin{array}{l}\text { Oddy, W. H.,Kendall, G. E.,Blair, E.,De Klerk, N. H.,Stanley, F. J.,Landau, L. I.,Silburn, S.,Zubrick, S. Breast feeding and cognitive } \\
\text { development in childhood: a prospective birth cohort study. Paediatr Perinat Epidemiol.2003;17:81-90. }\end{array}$ & IV \\
\hline 434 & $\begin{array}{l}\text { Oddy, W. H.,Robinson, M.,Kendall, G. E.,Li, J.,Zubrick, S. R.,Stanley, F. J. Breastfeeding and early child development: a prospective } \\
\text { cohort study. Acta Paediatr.2011;100:992-9. }\end{array}$ & IV \\
\hline 435 & $\begin{array}{l}\text { Oddy, W. H.,Sherriff, J. L. Breastfeeding, body mass index, asthma and atopy in children. Asia Pac J Public Health.2003;15 } \\
\text { Suppl:S15-7. }\end{array}$ & IV \\
\hline 436 & $\begin{array}{l}\text { O'Donovan,S. M.,O'B Hourihane J,Murray,D. M.,Kenny,L. C., Khashan,A. S.,Chaoimh,C. N.,Irvine,A. D.,Kiely,M. Neonatal adiposity } \\
\text { increases the risk of atopic dermatitis during the first year of life. J Allergy Clin Immunol.2015. }\end{array}$ & DV \\
\hline 437 & $\begin{array}{l}\text { Oelofse,A.,Van Raaij,J. M.,Benade,A. J.,Dhansay,M. A.,Tolboom,J. J.,Hautvast,J. G. The effect of a micronutrient-fortified } \\
\text { complementary food on micronutrient status, growth and development of 6- to 12-month-old disadvantaged urban South African } \\
\text { infants. Int J Food Sci Nutr.2003;54:399-407. }\end{array}$ & Country \\
\hline 438 & $\begin{array}{l}\text { Ong, K. K.,Emmett, P. M.,Noble, S.,Ness, A.,Dunger, D. B. Dietary energy intake at the age of } 4 \text { months predicts postnatal weight } \\
\text { gain and childhood body mass index. Pediatrics.2006;117:e503-8. }\end{array}$ & IV \\
\hline 439 & $\begin{array}{l}\text { Ong, K. K.,Preece, M. A.,Emmett, P. M.,Ahmed, M. L.,Dunger, D. B. Size at birth and early childhood growth in relation to maternal } \\
\text { smoking, parity and infant breast-feeding: longitudinal birth cohort study and analysis. Pediatr Res.2002;52:863-7. }\end{array}$ & IV \\
\hline 440 & $\begin{array}{l}\text { Onyango, A. W.,Borghi, E.,de Onis, M.,Casanovas Mdel, C.,Garza, C. Complementary feeding and attained linear growth among 6- } \\
\text { 23-month-old children. Public Health Nutr.2014;17:1975-83. }\end{array}$ & Design \\
\hline 441 & $\begin{array}{l}\text { Ou, X.,Andres, A.,Cleves, M. A.,Pivik, R. T.,Snow, J. H.,Ding, Z.,Badger, T. M. Sex-specific association between infant diet and } \\
\text { white matter integrity in 8-y-old children. Pediatr Res.2014;76:535-43. }\end{array}$ & IV \\
\hline 442 & $\begin{array}{l}\text { Ounsted, M. K.,Moar, V. A.,Scott, A. Large-for-dates babies at the age of four years: health, handicap and developmental status. } \\
\text { Early Hum Dev.1983;9:9-19. }\end{array}$ & IV \\
\hline 443 & Pachucki MA. Food pattern analysis over time: unhealthful eating trajectories predict obesity. Int J Obes (Lond).2012;36:686-94. & Age \\
\hline 444 & $\begin{array}{l}\text { Parry, J. E.,Ip, D. K.,Chau, P. Y.,Wu, K. M.,Tarrant, M. Predictors and consequences of in-hospital formula supplementation for } \\
\text { healthy breastfeeding newborns. J Hum Lact.2013;29:527-36. }\end{array}$ & IV, DV \\
\hline
\end{tabular}




\begin{tabular}{|c|c|c|}
\hline 445 & Parsons, T. J.,Power, C.,Manor, O. Infant feeding and obesity through the lifecourse. Arch Dis Child.2003;88:793-4. & IV \\
\hline 446 & $\begin{array}{l}\text { Patsourou, A.,Konstantinides, T.,Mantadakis, E.,Tsalkidis, A.,Zarras, C.,Balaska, A.,Simopoulos, K.,Chatzimichael, A. Growth of } \\
\text { exclusively breastfed and self-weaned children of Greece aged 0-36 months. Breastfeed Med.2012;7:521-5. }\end{array}$ & IV \\
\hline 447 & $\begin{array}{l}\text { Paul IM,Savage JS,Anzman SL,Beiler JS,Marini ME,Stokes JL,Birch LL. Preventing obesity during infancy: a pilot study. Obesity } \\
\text { (Silver Spring).2011;19:353-61. }\end{array}$ & IV \\
\hline 448 & Paul, A.,Whitehead, R. Infant feeding: the weighting game. Community Outlook.1986:11-7. & Design \\
\hline 449 & Paul, S. P.,Smith, B. A.,Taylor, T. M.,Walker, J. Take with a grain of salt. Clin Chem.2013;59:348-51. & Design \\
\hline 450 & $\begin{array}{l}\text { Peat,J. K.,Mihrshahi,S.,Kemp,A. S.,Marks,G. B.,Tovey,E. R.,Webb,K.,Mellis,C. M.,Leeder,S. R. Three-year outcomes of dietary fatty } \\
\text { acid modification and house dust mite reduction in the Childhood Asthma Prevention Study. J Allergy Clin Immunol.2004;114:807- } \\
\text { 13. }\end{array}$ & IV, DV \\
\hline 451 & $\begin{array}{l}\text { Penny,M. E.,Creed-Kanashiro,H. M.,Robert,R. C.,Narro,M. R.,Caulfield,L. E.,Black,R. E. Effectiveness of an educational intervention } \\
\text { delivered through the health services to improve nutrition in young children: a cluster-randomised controlled trial. } \\
\text { Lancet.2005;365:1863-72. }\end{array}$ & IV \\
\hline 452 & $\begin{array}{l}\text { Penrod,J. C.,Anderson,K.,Acosta,P. B. Impact on iron status of introducing cow's milk in the second six months of life. J Pediatr } \\
\text { Gastroenterol Nutr.1990;10:462-7. }\end{array}$ & IV, DV \\
\hline 453 & $\begin{array}{l}\text { Perez-Escamilla, R.,Cohen, R. J.,Brown, K. H.,Rivera, L. L.,Canahuati, J.,Dewey, K. G. Maternal anthropometric status and lactation } \\
\text { performance in a low-income Honduran population: evidence for the role of infants. Am J Clin Nutr.1995;61:528-34. }\end{array}$ & IV, DV \\
\hline 454 & Persson LA. Infant feeding and growth--a longitudinal study in three Swedish communities. Ann Hum Biol.1985;12:41-52. & IV \\
\hline 455 & $\begin{array}{l}\text { Persson,L. A.,Lundstrom,M.,Lonnerdal,B.,Hernell,O. Are weaning foods causing impaired iron and zinc status in 1-year-old Swedish } \\
\text { infants? A cohort study. Acta Paediatr.1998;87:618-22. }\end{array}$ & IV, DV \\
\hline 456 & $\begin{array}{l}\text { Peters,R. L.,Allen,K. J.,Dharmage,S. C.,Lodge,C. J.,Koplin,J. J.,Ponsonby,A. L.,Wake,M.,Lowe,A. J.,Tang,M. L.,Matheson,M. } \\
\text { C.,Gurrin,L. C. Differential factors associated with challenge-proven food allergy phenotypes in a population cohort of infants: a } \\
\text { latent class analysis. Clin Exp Allergy.2015;45:953-63. }\end{array}$ & DV \\
\hline 457 & $\begin{array}{l}\text { Pfluger M,Winkler C,Hummel S,Ziegler AG. Early infant diet in children at high risk for type } 1 \text { diabetes. Horm Metab } \\
\text { Res.2010;42:143-8. }\end{array}$ & IV \\
\hline 458 & $\begin{array}{l}\text { Pham,V. P.,Nguyen,V. H.,Salvignol,B.,Treche,S.,Wieringa,F. T.,Dijkhuizen,M. A.,Nguyen,C. K.,Pham,D. T.,Schwartz,H.,Berger,J. A } \\
\text { six-month intervention with two different types of micronutrient-fortified complementary foods had distinct short- and long-term } \\
\text { effects on linear and ponderal growth of Vietnamese infants. J Nutr.2012;142:1735-40. }\end{array}$ & Country \\
\hline 459 & $\begin{array}{l}\text { Phu,P. V.,Hoan,N. V.,Salvignol,B.,Treche,S.,Wieringa,F. T.,Khan,N. C.,Tuong,P. D.,Berger,J. Complementary foods fortified with } \\
\text { micronutrients prevent iron deficiency and anemia in Vietnamese infants. J Nutr.2010;140:2241-7. }\end{array}$ & DV \\
\hline 460 & $\begin{array}{l}\text { Piemontese, P.,Gianni, M. L.,Braegger, C. P.,Chirico, G.,Gruber, C., Riedler, J.,Arslanoglu, S.,van Stuijvenberg, M.,Boehm, } \\
\text { G.,Jelinek, J.,Roggero, P. Tolerance and safety evaluation in a large cohort of healthy infants fed an innovative prebiotic formula: a } \\
\text { randomized controlled trial. PLoS One.2011;6:e28010. }\end{array}$ & IV \\
\hline 461 & $\begin{array}{l}\text { Pimpin, L.,Jebb, S.,Johnson, L.,Wardle, J.,Ambrosini, G. L. Dietary protein intake is associated with body mass index and weight up } \\
\text { to } 5 \text { y of age in a prospective cohort of twins. Am J Clin Nutr.2016;103:389-97. }\end{array}$ & IV \\
\hline
\end{tabular}




\begin{tabular}{|c|c|c|}
\hline 462 & $\begin{array}{l}\text { Pivik, R. T.,Andres, A.,Badger, T. M. Effects of diet on early stage cortical perception and discrimination of syllables differing in } \\
\text { voice-onset time: a longitudinal ERP study in } 3 \text { and } 6 \text { month old infants. Brain Lang.2012;120:27-41. }\end{array}$ & IV \\
\hline 463 & $\begin{array}{l}\text { Pivik, R. T.,Dykman, R. A.,Jing, H.,Gilchrist, J. M.,Badger, T. M. The influence of infant diet on early developmental changes in } \\
\text { processing human voice speech stimuli: ERP variations in breast and milk formula-fed infants at } 3 \text { and } 6 \text { months after birth. Dev } \\
\text { Neuropsychol.2007;31:279-335. }\end{array}$ & IV \\
\hline 464 & $\begin{array}{l}\text { Piwoz, E. G.,Black, R. E.,Lopez de Romana, G.,Creed de Kanashiro, H.,Brown, K. H. The relationship between infants' preceding } \\
\text { appetite, illness, and growth performance and mothers' subsequent feeding practice decisions. Soc Sci Med.1994;39:851-60. }\end{array}$ & IV, DV \\
\hline 465 & $\begin{array}{l}\text { Piwoz, E. G., Creed de Kanashiro, H.,Lopez de Romana, G.,Black, R. E.,Brown, K. H. Within- and between-individual variation in } \\
\text { energy intakes by low-income Peruvian infants. Eur J Clin Nutr.1994;48:333-40. }\end{array}$ & IV, DV \\
\hline 466 & $\begin{array}{l}\text { Piwoz,E. G.,Lopez de Romana,G.,Creed de Kanashiro,H.,Black,R. E.,Brown,K. H. Indicators for monitoring the growth of peruvian } \\
\text { infants: weight and length gain vs attained weight and length. Am J Public Health.1994;84:1132-8. }\end{array}$ & IV \\
\hline 467 & $\begin{array}{l}\text { Plagemann, A.,Harder, T.,Kohlhoff, R.,Fahrenkrog, S.,Rodekamp, E.,Franke, K.,Dudenhausen, J. W. Impact of early neonatal } \\
\text { breast-feeding on psychomotor and neuropsychological development in children of diabetic mothers. Diabetes Care.2005;28:573-8. }\end{array}$ & IV \\
\hline 468 & $\begin{array}{l}\text { Plonka, K. A.,Pukallus, M. L.,Barnett, A.,Holcombe, T. F.,Walsh, L. J.,Seow, W. K. A controlled, longitudinal study of home visits } \\
\text { compared to telephone contacts to prevent early childhood caries. Int J Paediatr Dent.2013;23:23-31. }\end{array}$ & DV \\
\hline 469 & $\begin{array}{l}\text { Poh,Bee Koon,Ng,Boon Koon,Siti Haslinda,Mohd Din,Nik Shanita,Safii,Wong,Jyh Eiin,Budin,Siti Balkis,Ruzita,Abd Talib,Ng,Lai } \\
\text { Oon,Khouw,Ilse,Norimah,A. Karim. Nutritional status and dietary intakes of children aged } 6 \text { months to } 12 \text { years: findings of the } \\
\text { Nutrition Survey of Malaysian Children (SEANUTS Malaysia). British Journal of Nutrition.2013;110:S21-35 1p. }\end{array}$ & Design, IV \\
\hline 470 & $\begin{array}{l}\text { Polańska, K.,Muszyński, P.,Sobala, W.,Dziewirska, E.,Merecz-Kot, D.,Hanke, W. Maternal lifestyle during pregnancy and child } \\
\text { psychomotor development - Polish Mother and Child Cohort study. Early Human Development.2015;91:317-325. }\end{array}$ & IV \\
\hline 471 & $\begin{array}{l}\text { Pollitt, E.,Gorman, K. S.,Engle, P. L.,Rivera, J. A.,Martorell, R. Nutrition in early life and the fulfillment of intellectual potential. J } \\
\text { Nutr.1995;125:1111s-1118s. }\end{array}$ & Design, IV \\
\hline 472 & $\begin{array}{l}\text { Pollitt, E.,Watkins, W. E.,Husaini, M. A. Three-month nutritional supplementation in Indonesian infants and toddlers benefits memory } \\
\text { function } 8 \text { y later. Am J Clin Nutr.1997;66:1357-63. }\end{array}$ & Country \\
\hline 473 & Potur, A. H.,Kalmaz, N. An investigation into feeding errors of 0-4-month-old infants. J Trop Pediatr.1995;41:120-2. & $\begin{array}{l}\text { Design, } \\
\text { IV,DV }\end{array}$ \\
\hline 474 & Potur, A. H.,Kalmaz, N. An investigation into feeding errors of 0-4-month-old infants. J Trop Pediatr.1996;42:173-5. & $\begin{array}{l}\text { Design, } \\
\text { IV,DV }\end{array}$ \\
\hline 475 & $\begin{array}{l}\text { Prentice P,Koulman A,Matthews L,Acerini CL,Ong KK,Dunger DB. Lipidomic analyses, breast- and formula-feeding, and growth in } \\
\text { infants. J Pediatr.2015;166:276-81.e6. }\end{array}$ & IV \\
\hline 476 & $\begin{array}{l}\text { Pridham, K. F. Infant feeding and anticipatory care: supporting the adaptation of parents to their new babies. Matern Child Nurs } \\
\text { J.1981;10:111-26. }\end{array}$ & Design \\
\hline 477 & $\begin{array}{l}\text { Priego, T.,Sanchez, J.,Pico, C.,Ahrens, W.,Bammann, K.,De Henauw, S.,Fraterman, A.,lacoviello, L.,Lissner, L.,Molnar, D.,Moreno, } \\
\text { L. A.,Siani, A.,Tornaritis, M.,Veidebaum, T.,Palou, A. Influence of breastfeeding on blood-cell transcript-based biomarkers of health } \\
\text { in children. Pediatr Obes.2014;9:463-70. }\end{array}$ & IV \\
\hline 478 & $\begin{array}{l}\text { Prodam, F.,Roccio, M.,Trovato, L.,Ricotti, R.,Moia, S.,Giglione, E.,Petri, A.,Walker, G. E.,Bellone, S.,Bona, G. Adiponectin oligomers } \\
\text { are similarly distributed in adequate-for-gestational-age obese children irrespective of feeding in their first year. Pediatr }\end{array}$ & IV, DV \\
\hline
\end{tabular}




\begin{tabular}{|c|c|c|}
\hline & Res.2015;77:808-13. & \\
\hline 479 & $\begin{array}{l}\text { Puri, R.,Chawla, P.,Sharma, M.,Pershad, D. Impact of an on-going supplementary feeding programme on the mental abilities of } \\
\text { children. Indian J Pediatr.1984;51:653-7. }\end{array}$ & IV \\
\hline 480 & $\begin{array}{l}\text { Purwestri, R. C.,Scherbaum, V.,Inayati, D. A.,Wirawan, N. N.,Suryantan, J.,Bloem, M. A.,Pangaribuan, R. V.,Stuetz, W.,Hoffmann, } \\
\text { V.,Qaim, M.,Biesalski, H. K.,Bellows, A. C. Supplementary feeding with locally-produced Ready-to-Use Food (RUF) for mildly } \\
\text { wasted children on Nias Island, Indonesia: comparison of daily and weekly program outcomes. Asia Pac J Clin Nutr.2012;21:374-9. }\end{array}$ & $\begin{array}{l}\text { Health } \\
\text { status, IV }\end{array}$ \\
\hline 481 & $\begin{array}{l}\text { Qureshi, B.,Morgan, J. B.,Kimer, A. C.,Donaldson, D.,Dickerson, J. W. Feeding practices and birth weights of infants in Southall, } \\
\text { Middlesex. J R Soc Health.1988;108:77-80. }\end{array}$ & IV, DV \\
\hline 482 & $\begin{array}{l}\text { Radhakrishna,K. V.,Hemalatha,R.,Geddam,J. J.,Kumar,P. A.,Balakrishna,N.,Shatrugna,V. Effectiveness of zinc supplementation to } \\
\text { full term normal infants: a community based double blind, randomized, controlled, clinical trial. PLoS One.2013;8:e61486. }\end{array}$ & IV \\
\hline 483 & $\begin{array}{l}\text { Ramokolo, V.,Lombard, C.,Chhagan, M.,Engebretsen, I. M.,Doherty, T.,Goga, A. E.,Fadnes, L. T.,Zembe, W.,Jackson, D. J.,Van } \\
\text { den Broeck, J. Effects of early feeding on growth velocity and overweight/obesity in a cohort of HIV unexposed South African infants } \\
\text { and children. Int Breastfeed J.2015;10:14. }\end{array}$ & IV \\
\hline 484 & Rao,S.,Rajpathak,V. Breastfeeding and weaning practices in relation to nutritional status of infants. Indian Pediatr.1992;29:1533-9. & IV, DV \\
\hline 485 & Rapley, G. Talking about weaning. Community Pract.2011;84:40-1. & Design \\
\hline 486 & $\begin{array}{l}\text { Rask-Nissila,L.,Jokinen,E.,Terho,P.,Tammi,A.,Hakanen,M.,Ronnemaa,T.,Viikari,J.,Seppanen,R.,Valimaki,I,Helenius,H.,Simell,O. } \\
\text { Effects of diet on the neurologic development of children at } 5 \text { years of age: the STRIP project. J Pediatr.2002;140:328-33. }\end{array}$ & IV \\
\hline 487 & $\begin{array}{l}\text { Ravelli, A. C.,van der Meulen, J. H.,Osmond, C.,Barker, D. J.,Bleker, O. P. Infant feeding and adult glucose tolerance, lipid profile, } \\
\text { blood pressure, and obesity. Arch Dis Child.2000;82:248-52. }\end{array}$ & IV \\
\hline 488 & $\begin{array}{l}\text { Reifsnider, E. Reversing growth deficiency in children: the effect of a community-based intervention. J Pediatr Health } \\
\text { Care.1998;12:305-12. }\end{array}$ & $\begin{array}{l}\text { Health } \\
\text { status, IV }\end{array}$ \\
\hline 489 & $\begin{array}{l}\text { Requejo,A. M.,Navia,B.,Ortega,R. M.,Lopez-Sobaler,A. M.,Quintas,E.,Gaspar,M. J.,Osorio,O. The age at which meat is first included } \\
\text { in the diet affects the incidence of iron deficiency and ferropenic anaemia in a group of pre-school children from Madrid. Int J Vitam } \\
\text { Nutr Res.1999;69:127-31. }\end{array}$ & Design \\
\hline 490 & $\begin{array}{l}\text { Richards, M.,Wadsworth, M.,Rahimi-Foroushani, A.,Hardy, R.,Kuh, D.,Paul, A. Infant nutrition and cognitive development in the first } \\
\text { offspring of a national UK birth cohort. Dev Med Child Neurol.1998;40:163-7. }\end{array}$ & IV \\
\hline 491 & $\begin{array}{l}\text { Rifas-Shiman SL,Sherry B,Scanlon K,Birch LL,Gillman MW,Taveras EM. Does maternal feeding restriction lead to childhood obesity } \\
\text { in a prospective cohort study?. Arch Dis Child.2011;96:265-9. }\end{array}$ & IV \\
\hline 492 & $\begin{array}{l}\text { Rios-Castillo, I.,Cerezo, S.,Corvalan, C.,Martinez, M.,Kain, J. Risk factors during the prenatal period and the first year of life } \\
\text { associated with overweight in 7-year-old low-income Chilean children. Matern Child Nutr.2015;11:595-605. }\end{array}$ & Design \\
\hline 493 & $\begin{array}{l}\text { Rivera,J. A.,Habicht,J. P. Effect of supplementary feeding on the prevention of mild-to-moderate wasting in conditions of endemic } \\
\text { malnutrition in Guatemala. Bull World Health Organ.2002;80:926-32. }\end{array}$ & IV \\
\hline 494 & Roberts, D. W. Growth of breast fed and bottle fed infants. N Z Med J.1980;92:45-6. & IV \\
\hline 495 & Robinson, S. M.,Simmonds, S. J.,Jameson, K. A.,Syddall, H. E.,Dennison, E. M.,Cooper, C.,Sayer, A. A. Muscle strength in older & IV \\
\hline
\end{tabular}




\begin{tabular}{|c|c|c|}
\hline & community-dwelling men is related to type of milk feeding in infancy. J Gerontol A Biol Sci Med Sci.2012;67:990-6. & \\
\hline 496 & $\begin{array}{l}\text { Robinson, S.,Ntani, G.,Simmonds, S.,Syddall, H.,Dennison, E.,Sayer, A. A.,Barker, D.,Cooper, C. Type of milk feeding in infancy } \\
\text { and health behaviours in adult life: findings from the Hertfordshire Cohort Study. Br J Nutr.2013;109:1114-22. }\end{array}$ & IV, DV \\
\hline 497 & Rogan, W. J.,Gladen, B. C. Breast-feeding and cognitive development. Early Hum Dev.1993;31:181-93. & IV \\
\hline 498 & $\begin{array}{l}\text { Rolland-Cachera,M. F.,Maillot,M.,Deheeger,M.,Souberbielle,J. C.,Peneau,S.,Hercberg,S. Association of nutrition in early life with } \\
\text { body fat and serum leptin at adult age. Int J Obes (Lond).2013;37:1116-22. }\end{array}$ & IV \\
\hline 499 & $\begin{array}{l}\text { Rosado,J. L.,Lopez,P.,Garcia,O. P.,Alatorre,J.,Alvarado,C. Effectiveness of the nutritional supplement used in the Mexican } \\
\text { Oportunidades programme on growth, anaemia, morbidity and cognitive development in children aged 12-24 months. Public Health } \\
\text { Nutr.2011;14:931-7. }\end{array}$ & IV \\
\hline 500 & $\begin{array}{l}\text { Routi, T.,Ronnemaa, T.,Lapinleimu, H.,Salo, P.,Viikari, J.,Leino, A.,Valimaki, I.,Jokinen, E.,Simell, O. Effect of weaning on serum } \\
\text { lipoprotein(a) concentration: the STRIP baby study. Pediatr Res.1995;38:522-7. }\end{array}$ & IV, DV \\
\hline 501 & $\begin{array}{l}\text { Rowland, M. G. The "why" and "when" of introducing food to infants: growth in young breast-fed infants and some nutritional } \\
\text { implications. Am J Clin Nutr.1985;41:459-63. }\end{array}$ & Design \\
\hline 502 & $\begin{array}{l}\text { Roy, S. K.,Fuchs, G. J.,Mahmud, Z.,Ara, G.,Islam, S.,Shafique, S.,Akter, S. S.,Chakraborty, B. Intensive nutrition education with or } \\
\text { without supplementary feeding improves the nutritional status of moderately-malnourished children in Bangladesh. J Health Popul } \\
\text { Nutr.2005;23:320-30. }\end{array}$ & $\begin{array}{l}\text { Health } \\
\text { status }\end{array}$ \\
\hline 503 & $\begin{array}{l}\text { Rudnicka, A. R.,Owen, C. G.,Richards, M.,Wadsworth, M. E.,Strachan, D. P. Effect of breastfeeding and sociodemographic factors } \\
\text { on visual outcome in childhood and adolescence. Am J Clin Nutr.2008;87:1392-9. }\end{array}$ & IV \\
\hline 504 & Rudy,C. A. Vegetarian diets for children. Pediatr Nurs.1984;10:329-33. & Design \\
\hline 505 & $\begin{array}{l}\text { Ruel, M. T.,Rivera, J.,Habicht, J. P.,Martorell, R. Differential response to early nutrition supplementation: long-term effects on height } \\
\text { at adolescence. Int J Epidemiol.1995;24:404-12. }\end{array}$ & IV, Date \\
\hline 506 & $\begin{array}{l}\text { Russell, C. G.,Taki, S.,Azadi, L.,Campbell, K. J.,Laws, R.,Elliott, R.,Denney-Wilson, E. A qualitative study of the infant feeding } \\
\text { beliefs and behaviours of mothers with low educational attainment. BMC Pediatr.2016;16:69. }\end{array}$ & DV \\
\hline 507 & Ryan, A. S.,Gussler, J. D. Biocultural factors affecting infant feeding and growth. Med Anthropol.1985;9:93-5. & Design \\
\hline 508 & $\begin{array}{l}\text { Rzehak, P.,Sausenthaler, S.,Koletzko, S., Reinhardt, D.,von Berg, A.,Kramer, U.,Berdel, D.,Bollrath, C.,Grubl, A.,Bauer, C. } \\
\text { P.,Wichmann, H. E.,Heinrich, J. Long-term effects of hydrolyzed protein infant formulas on growth--extended follow-up to } 10 \text { y of } \\
\text { age: results from the German Infant Nutritional Intervention (GINI) study. Am J Clin Nutr.2011;94:1803s-1807s. }\end{array}$ & IV \\
\hline 509 & $\begin{array}{l}\text { Saco-Pollitt, C.,Triana, N.,Harahap, H.,Husaini, M.,Jahari, A. B.,Pollitt, E. The eco-cultural context of the undernourished children in } \\
\text { a study on the effects of early supplementary feeding in Indonesia. Eur J Clin Nutr.2000;54 Suppl 2:S11-5. }\end{array}$ & $\begin{array}{l}\text { Design, } \\
\text { Health } \\
\text { status }\end{array}$ \\
\hline 510 & $\begin{array}{l}\text { Sacrey, L. A., Karl, J. M.,Whishaw, I. Q. Development of rotational movements, hand shaping, and accuracy in advance and } \\
\text { withdrawal for the reach-to-eat movement in human infants aged 6-12 months. Infant Behav Dev.2012;35:543-60. }\end{array}$ & IV, DV \\
\hline 511 & $\begin{array}{l}\text { Saha, K. K.,Frongillo, E. A.,Alam, D. S.,Arifeen, S. E.,Persson, L. A., Rasmussen, K. M. Appropriate infant feeding practices result in } \\
\text { better growth of infants and young children in rural Bangladesh. Am J Clin Nutr.2008;87:1852-9. }\end{array}$ & IV \\
\hline
\end{tabular}




\begin{tabular}{|c|c|c|}
\hline 512 & $\begin{array}{l}\text { Samuel, T. M.,Thomas, T.,Bhat, S.,Kurpad, A. V. Are infants born in baby-friendly hospitals being exclusively breastfed until } 6 \\
\text { months of age?. Eur J Clin Nutr.2012;66:459-65. }\end{array}$ & IV \\
\hline 513 & Saner G,Dagoglu T,Uzkan I,Neyzi O. Promotion of breastfeeding in the postpartum mother. Turk J Pediatr.1985;27:63-8. & IV \\
\hline 514 & Sanger, R. G.,Bystrom, E. B. Breast feeding: does it affect oral facial growth?. Dent Hyg (Chic).1982;56:44-7. & Design \\
\hline 515 & $\begin{array}{l}\text { Santos, I. S.,Matijasevich, A.,Assuncao, M. C.,Valle, N. C.,Horta, B. L.,Goncalves, H. D.,Gigante, D. P.,Martines, J. C.,Pelto, } \\
\text { G.,Victora, C. G. Promotion of Weight Gain in Early Childhood Does Not Increase Metabolic Risk in Adolescents: A 15-Year Follow- } \\
\text { Up of a Cluster-Randomized Controlled Trial. J Nutr.2015;145:2749-55. }\end{array}$ & IV \\
\hline 516 & $\begin{array}{l}\text { Santos,I.,Victora,C. G.,Martines,J.,Goncalves,H.,Gigante,D. P.,Valle,N. J.,Pelto,G. Nutrition counseling increases weight gain } \\
\text { among Brazilian children. J Nutr.2001;131:2866-73. }\end{array}$ & IV \\
\hline 517 & $\begin{array}{l}\text { Satter, Ma,Jabin, Sa,Abedin, N.,Mf, Mf,Parvin, R.,Dhali, M.,Amin, Mz. Development and Evaluation of Weaning Foods Using Locally } \\
\text { Available Nutritious Fruits in Bangladesh. Malaysian Journal of Nutrition.2014;20:83-92. }\end{array}$ & $\begin{array}{l}\text { Design, IV, } \\
\text { DV }\end{array}$ \\
\hline 518 & $\begin{array}{l}\text { Savino, F.,Maccario, S.,Cresi, F.,Grasso, G.,Oggero, R.,Silvestro, L.,Mussa, G. C. Bioimpedance vector analysis in breastfed and } \\
\text { formula-fed infants in the first six months of life. Adv Exp Med Biol.2004;554:501-4. }\end{array}$ & Design, IV \\
\hline 519 & $\begin{array}{l}\text { Savino, F.,Sorrenti, M.,Benetti, S.,Lupica, M. M.,Liguori, S. A.,Oggero, R. Resistin and leptin in breast milk and infants in early life. } \\
\text { Early Hum Dev.2012;88:779-82. }\end{array}$ & Design, IV \\
\hline 520 & Sawley, L. Infant feeding. Nursing (Lond).1989;3:18-23. & Design \\
\hline 521 & $\begin{array}{l}\text { Sazawal,S.,Dhingra,P.,Dhingra,U.,Gupta,S.,Iyengar,V.,Menon,V. P.,Sarkar,A.,Black,R. E. Compliance with home-based fortification } \\
\text { strategies for delivery of iron and zinc: its effect on haematological and growth markers among 6-24 months old children in north } \\
\text { India. J Health Popul Nutr.2014;32:217-26. }\end{array}$ & Country \\
\hline 522 & $\begin{array}{l}\text { Scaglioni, S.,Agostoni, C.,Notaris, R. D.,Radaelli, G.,Radice, N.,Valenti, M.,Giovannini, M.,Riva, E. Early macronutrient intake and } \\
\text { overweight at five years of age. Int J Obes Relat Metab Disord.2000;24:777-81. }\end{array}$ & IV \\
\hline 523 & $\begin{array}{l}\text { Scheiwe, A.,Hardy, R.,Watt, R. G. Four-year follow-up of a randomized controlled trial of a social support intervention on infant } \\
\text { feeding practices. Matern Child Nutr.2010;6:328-37. }\end{array}$ & IV \\
\hline 524 & Schmalz, M.,Boos, K.,Schmalz, G.,Huntington, M. K. Failure to thrive. J Fam Pract.2009;58:539-44. & Design \\
\hline 525 & $\begin{array}{l}\text { Schmidt,M. K.,Muslimatun,S.,West,C. E.,Schultink,W.,Gross,R.,Hautvast,J. G. Nutritional status and linear growth of Indonesian } \\
\text { infants in west java are determined more by prenatal environment than by postnatal factors. J Nutr.2002;132:2202-7. }\end{array}$ & Country \\
\hline 526 & $\begin{array}{l}\text { Schroeder, D. G.,Martorell, R.,Rivera, J. A., Ruel, M. T.,Habicht, J. P. Age differences in the impact of nutritional supplementation on } \\
\text { growth. J Nutr.1995;125:1051s-1059s. }\end{array}$ & IV \\
\hline 527 & $\begin{array}{l}\text { Schroeder, N.,Rushovich, B.,Bartlett, E.,Sharma, S.,Gittelsohn, J.,Caballero, B. Early Obesity Prevention: A Randomized Trial of a } \\
\text { Practice-Based Intervention in 0-24-Month Infants. J Obes.2015;2015:795859. }\end{array}$ & IV \\
\hline 528 & $\begin{array}{l}\text { Schwartz, R.,Vigo, A.,de Oliveira, L. D.,Justo Giugliani, E. R. The Effect of a Pro-Breastfeeding and Healthy Complementary } \\
\text { Feeding Intervention Targeting Adolescent Mothers and Grandmothers on Growth and Prevalence of Overweight of Preschool } \\
\text { Children. PLoS One.2015;10:e0131884. }\end{array}$ & IV \\
\hline 529 & Scott, J. A.,Binns, C. W.,Graham, K. I.,Oddy, W. H. Predictors of the early introduction of solid foods in infants: results of a cohort & DV \\
\hline
\end{tabular}




\begin{tabular}{|c|c|c|}
\hline 530 & $\begin{array}{l}\text { Scott, J. A.,Dashti, M.,Al-Sughayer, M.,Edwards, C. A. Timing and Determinants of the Introduction of Complementary Foods in } \\
\text { Kuwait: Results of a Prospective Cohort Study. J Hum Lact.2015;31:467-73. }\end{array}$ & DV \\
\hline 531 & Scully, T. Obesity. Nature.2014;508:S49. & Design \\
\hline 532 & Seal, N.,Broome, M. E. Prepregnancy Body Mass Index and Feeding Practices in Relation to Infants' Growth. J Nurse Pract.2013;9. & Design \\
\hline 533 & $\begin{array}{l}\text { Seksaria,S. A.,Sheth,M. K. Mass media as a means to bring about behavioral changes in infant and young child feeding practices } \\
\text { amongst tribal mothers of Chikhli taluka, Gujarat. Indian Journal of Public Health Research and Development.2015;6:113-118. }\end{array}$ & Design, IV \\
\hline 534 & $\begin{array}{l}\text { Sellen, D. W.,Thompson, A. L.,Hruschka, D. J.,Stein, A. D.,Martorell, R. Early determinants of non-exclusive breastfeeding among } \\
\text { Guatemalan infants. Adv Exp Med Biol.2004;554:299-301. }\end{array}$ & IV, DV \\
\hline 535 & $\begin{array}{l}\text { Senarath, U.,Godakandage, S. S.,Jayawickrama, H.,Siriwardena, I.,Dibley, M. J. Determinants of inappropriate complementary } \\
\text { feeding practices in young children in Sri Lanka: secondary data analysis of Demographic and Health Survey 2006-2007. Matern } \\
\text { Child Nutr.2012;8 Suppl 1:60-77. }\end{array}$ & Design,DV \\
\hline 536 & Sethi, V.,Kashyap, S.,Seth, V. Effect of nutrition education of mothers on infant feeding practices. Indian J Pediatr.2003;70:463-6. & IV \\
\hline 537 & $\begin{array}{l}\text { Sezer,R. G.,Aydemir,G.,Akcan,A. B.,Bayoglu,D. S.,Guran,T.,Bozaykut,A. Effect of breastfeeding on serum zinc levels and growth in } \\
\text { healthy infants. Breastfeed Med.2013;8:159-63. }\end{array}$ & Design \\
\hline 538 & $\begin{array}{l}\text { Shaternikov,V. A.,Fateeva,E. M.,Chernikov,M. N. Protein nutrition in early infancy and subsequent periods: its effect on further } \\
\text { development. Bibl Nutr Dieta.1982:95-111. }\end{array}$ & Design \\
\hline 539 & $\begin{array}{l}\text { Shehadeh, N., Weitzer-Kish, H.,Shamir, R., Shihab, S., Weiss, R. Impact of early postnatal weight gain and feeding patterns on body } \\
\text { mass index in adolescence. J Pediatr Endocrinol Metab.2008;21:9-15. }\end{array}$ & IV \\
\hline 540 & $\begin{array}{l}\text { Shi,L.,Zhang,J.,Wang,Y.,Caulfield,L. E.,Guyer,B. Effectiveness of an educational intervention on complementary feeding practices } \\
\text { and growth in rural China: a cluster randomised controlled trial. Public Health Nutr.2010;13:556-65. }\end{array}$ & IV \\
\hline 541 & $\begin{array}{l}\text { Shim, J. E.,Kim, J.,Mathai, R. A. Associations of infant feeding practices and picky eating behaviors of preschool children. J Am Diet } \\
\text { Assoc.2011;111:1363-8. }\end{array}$ & DV \\
\hline 542 & $\begin{array}{l}\text { Simell, O.,Niinikoski, H.,Viikari, J.,Rask-Nissila, L., Tammi, A., Ronnemaa, T. Cardiovascular disease risk factors in young children in } \\
\text { the STRIP baby project. Special Turku coronary Risk factor Intervention Project for children. Ann Med.1999;31 Suppl 1:55-61. }\end{array}$ & IV \\
\hline 543 & $\begin{array}{l}\text { Simondon, K. B.,Gartner, A.,Berger, J.,Cornu, A.,Massamba, J. P.,San Miguel, J. L.,Ly, C.,Missotte, I.,Simondon, F.,Traissac, } \\
\text { P.,Delpeuch, F.,Maire, B. Effect of early, short-term supplementation on weight and linear growth of 4-7-mo-old infants in developing } \\
\text { countries: a four-country randomized trial. Am J Clin Nutr.1996;64:537-45. }\end{array}$ & IV,Country \\
\hline 544 & $\begin{array}{l}\text { Singhal A,Cole TJ,Fewtrell M,Kennedy K,Stephenson T,Elias-Jones A,Lucas A. Promotion of faster weight gain in infants born small } \\
\text { for gestational age: is there an adverse effect on later blood pressure?. Circulation.2007;115:213-20. }\end{array}$ & $\begin{array}{l}\text { Health } \\
\text { status, IV }\end{array}$ \\
\hline 545 & Singhal, A.,Lucas, A. Early origins of cardiovascular disease: is there a unifying hypothesis?. Lancet.2004;363:1642-5. & Design \\
\hline 546 & $\begin{array}{l}\text { Skau,J. K.,Touch,B.,Chhoun,C.,Chea,M.,Unni,U. S.,Makurat,J.,Filteau,S.,Wieringa,F. T.,Dijkhuizen,M. A.,Ritz,C.,Wells,J. } \\
\text { C.,Berger,J.,Friis,H.,Michaelsen,K. F.,Roos,N. Effects of animal source food and micronutrient fortification in complementary food } \\
\text { products on body composition, iron status, and linear growth: a randomized trial in Cambodia. Am J Clin Nutr.2015;101:742-51. }\end{array}$ & IV \\
\hline
\end{tabular}




\begin{tabular}{|c|c|c|}
\hline 547 & $\begin{array}{l}\text { Skledar, M. T.,Milosevic, M. Breastfeeding and time of complementary food introduction as predictors of obesity in children. Cent } \\
\text { Eur J Public Health.2015;23:26-31. }\end{array}$ & Design \\
\hline 548 & $\begin{array}{l}\text { Sloan, S.,Gildea, A.,Stewart, M.,Sneddon, H.,Iwaniec, D. Early weaning is related to weight and rate of weight gain in infancy. Child } \\
\text { Care Health Dev.2008;34:59-64. }\end{array}$ & Design \\
\hline 549 & $\begin{array}{l}\text { Snijders, B. E.,Thijs, C.,van Ree, R.,van den Brandt, P. A. Age at first introduction of cow milk products and other food products in } \\
\text { relation to infant atopic manifestations in the first } 2 \text { years of life: the KOALA Birth Cohort Study. Pediatrics.2008;122:e115-22. }\end{array}$ & DV \\
\hline 550 & $\begin{array}{l}\text { Soto-Ramirez, N.,Karmaus, W.,Zhang, H.,Davis, S.,Agarwal, S.,Albergottie, A. Modes of infant feeding and the occurrence of } \\
\text { coughing/wheezing in the first year of life. J Hum Lact.2013;29:71-80. }\end{array}$ & IV, DV \\
\hline 551 & Sparks, J. W. Fetal growth and diet. Mead Johnson Symp Perinat Dev Med.1984:21-7. & Design \\
\hline 552 & $\begin{array}{l}\text { Specker BL,Beck A,Kalkwarf H,Ho M. Randomized trial of varying mineral intake on total body bone mineral accretion during the first } \\
\text { year of life. Pediatrics.1997;99:E12. }\end{array}$ & IV \\
\hline 553 & $\begin{array}{l}\text { Spence,A. C.,Campbell,K. J.,Crawford,D. A.,McNaughton,S. A.,Hesketh,K. D. Mediators of improved child diet quality following a } \\
\text { health promotion intervention: the Melbourne InFANT Program. Int J Behav Nutr Phys Act.2014;11:137. }\end{array}$ & IV, DV \\
\hline 554 & $\begin{array}{l}\text { Sreedhara, M. S.,Banapurmath, C. R. A study of nutritional status of infants in relation to their complementary feeding practices. } \\
\text { Current Pediatric Research.2014;18:39-41. }\end{array}$ & Design \\
\hline 555 & Stahl, M. D.,Guida, D. A. Slow weight gain in the breast-fed infant: management options. Pediatr Nurs.1984;10:117-20, 164. & Design \\
\hline 556 & Stang,J. Improving the eating patterns of infants and toddlers. J Am Diet Assoc.2006;106:S7-9. & Design \\
\hline 557 & $\begin{array}{l}\text { Stifter, C. A.,Anzman-Frasca, S.,Birch, L. L.,Voegtline, K. Parent use of food to soothe infant/toddler distress and child weight status. } \\
\text { An exploratory study. Appetite.2011;57:693-9. }\end{array}$ & Design, IV \\
\hline 558 & $\begin{array}{l}\text { Strbak, V.,Skultetyova, M.,Hromadova, M.,Randuskova, A.,Macho, L. Late effects of breast-feeding and early weaning: seven-year } \\
\text { prospective study in children. Endocr Regul.1991;25:53-7. }\end{array}$ & IV \\
\hline 559 & $\begin{array}{l}\text { Stuff, J. E.,Garza, C.,Boutte, C.,Fraley, J. K.,Smith, E. O.,Klein, E. R.,Nichols, B. L. Sources of variance in milk and caloric intakes in } \\
\text { breast-fed infants: implications for lactation study design and interpretation. Am J Clin Nutr.1986;43:361-6. }\end{array}$ & Design, IV \\
\hline 560 & Stuff, J. E.,Nichols, B. L. Nutrient intake and growth performance of older infants fed human milk. J Pediatr.1989;115:959-68. & DV \\
\hline 561 & $\begin{array}{l}\text { Stunkard, A. J.,Berkowitz, R. I.,Schoeller, D.,Maislin, G.,Stallings, V. A. Predictors of body size in the first } 2 \text { y of life: a high-risk study } \\
\text { of human obesity. Int J Obes Relat Metab Disord.2004;28:503-13. }\end{array}$ & IV \\
\hline 562 & $\begin{array}{l}\text { Stunkard, A. J.,Berkowitz, R. I.,Stallings, V. A.,Schoeller, D. A. Energy intake, not energy output, is a determinant of body size in } \\
\text { infants. Am J Clin Nutr.1999;69:524-30. }\end{array}$ & IV \\
\hline 563 & $\begin{array}{l}\text { Super, C. M.,Herrera, M. G.,Mora, J. O. Long-term effects of food supplementation and psychosocial intervention on the physical } \\
\text { growth of Colombian infants at risk of malnutrition. Child Dev.1990;61:29-49. }\end{array}$ & IV \\
\hline 564 & $\begin{array}{l}\text { Svahn,J. C.,Feldl,F.,Raiha,N. C.,Koletzko,B.,Axelsson,I. E. Different quantities and quality of fat in milk products given to young } \\
\text { children: effects on long chain polyunsaturated fatty acids and trans fatty acids in plasma. Acta Paediatr.2002;91:20-9. }\end{array}$ & DV \\
\hline 565 & $\begin{array}{l}\text { Taitz, L. S.,Lukmanji, Z. Alterations in feeding patterns and rates of weight gain in South Yorkshire infants, 1971-1977. Hum } \\
\text { Biol.1981;53:313-20. }\end{array}$ & IV, Date \\
\hline
\end{tabular}




\begin{tabular}{|c|c|c|}
\hline 566 & Taitz, L. Feeding children in the first year of life. Midwife Health Visitor \& Community Nurse.1990;26:81-84. & Design \\
\hline 567 & Tann, S. P.,Wheeler, E. F. Food intakes and growth of young Chinese children in London. Community Med.1980;2:20-4. & IV, DV \\
\hline 568 & $\begin{array}{l}\text { Tantracheewathorn,S.,Lohajaroensub,S. Incidence and risk factors of iron deficiency anemia in term infants. J Med Assoc } \\
\text { Thai.2005;88:45-51. }\end{array}$ & IV, DV \\
\hline 569 & $\begin{array}{l}\text { Tanzer F,Gumuser C. A study of the growth of } 200 \text { newborn babies for a period of } 6 \text { months according to the type of nutrition. Ann } \\
\text { Trop Paediatr.1989;9:54-8. }\end{array}$ & IV \\
\hline 570 & $\begin{array}{l}\text { Tarrant, M.,Fong, D. Y.,Wu, K. M.,Lee, I. L.,Wong, E. M.,Sham, A.,Lam, C., Dodgson, J. E. Breastfeeding and weaning practices } \\
\text { among Hong Kong mothers: a prospective study. BMC Pregnancy Childbirth.2010;10:27. }\end{array}$ & DV \\
\hline 571 & $\begin{array}{l}\text { Taveras EM,Blackburn K,Gillman MW,Haines J,McDonald J,Price S,Oken E. First steps for mommy and me: a pilot intervention to } \\
\text { improve nutrition and physical activity behaviors of postpartum mothers and their infants. Matern Child Health J.2011;15:1217-27. }\end{array}$ & IV \\
\hline 572 & $\begin{array}{l}\text { Tawia, S. Breastfeeding, brain structure and function, cognitive development and educational attainment. Breastfeed } \\
\text { Rev.2013;21:15-20. }\end{array}$ & Design \\
\hline 573 & Tawia, S. Childhood obesity and being breastfed. Breastfeed Rev.2013;21:42-8. & Design \\
\hline 574 & $\begin{array}{l}\text { Taylor,A.,Redworth,E. W.,Morgan,J. B. Influence of diet on iron, copper, and zinc status in children under } 24 \text { months of age. Biol } \\
\text { Trace Elem Res.2004;97:197-214. }\end{array}$ & DV \\
\hline 575 & $\begin{array}{l}\text { Thakur, R.,Singh, M. G.,Chaudhary, S.,Manuja, N. Effect of mode of delivery and feeding practices on acquisition of oral } \\
\text { Streptococcus mutans in infants. Int J Paediatr Dent.2012;22:197-202. }\end{array}$ & DV \\
\hline 576 & $\begin{array}{l}\text { Theron, M.,Amissah, A.,Kleynhans, I. C.,Albertse, E.,Maclntyre, U. E. Inadequate dietary intake is not the cause of stunting amongst } \\
\text { young children living in an informal settlement in Gauteng and rural Limpopo Province in South Africa: the NutriGro study. Public } \\
\text { Health Nutr.2007;10:379-89. }\end{array}$ & Design \\
\hline 577 & $\begin{array}{l}\text { Thomson, J. L.,Tussing-Humphreys, L. M.,Goodman, M. H. Delta Healthy Sprouts: a randomized comparative effectiveness trial to } \\
\text { promote maternal weight control and reduce childhood obesity in the Mississippi Delta. Contemp Clin Trials.2014;38:82-91. }\end{array}$ & Design, IV \\
\hline 578 & $\begin{array}{l}\text { Thorisdottir, B.,Gunnarsdottir, I., Thorisdottir, A. V.,Palsson, G. I.,Halldorsson, T. I.,Thorsdottir, I. Nutrient intake in infancy and body } \\
\text { mass index at six years in two population-based cohorts recruited before and after revision of infant dietary recommendations. Ann } \\
\text { Nutr Metab.2013;63:145-51. }\end{array}$ & IV \\
\hline 579 & $\begin{array}{l}\text { Thorsdottir, I.,Gunnarsdottir, I.,Palsson, G. I. Birth weight, growth and feeding in infancy: relation to serum lipid concentration in } 12- \\
\text { month-old infants. Eur J Clin Nutr.2003;57:1479-85. }\end{array}$ & IV, DV \\
\hline 580 & $\begin{array}{l}\text { Timby, N.,Domellof, E.,Hernell, O.,Lonnerdal, B.,Domellof, M. Neurodevelopment, nutrition, and growth until } 12 \text { mo of age in infants } \\
\text { fed a low-energy, low-protein formula supplemented with bovine milk fat globule membranes: a randomized controlled trial. Am J } \\
\text { Clin Nutr.2014;99:860-8. }\end{array}$ & IV \\
\hline 581 & $\begin{array}{l}\text { Townsend, E.,Pitchford, N. J. Baby knows best? The impact of weaning style on food preferences and body mass index in early } \\
\text { childhood in a case-controlled sample. BMJ Open.2012;2:e000298. }\end{array}$ & IV \\
\hline 582 & $\begin{array}{l}\text { Tripathy, R.,Das, R. N.,Das, M. M.,Parija, A. C. Growth in the first year in children following IAP Policy on Infant Feeding. Indian } \\
\text { Pediatr.2000;37:1051-9. }\end{array}$ & IV \\
\hline
\end{tabular}




\begin{tabular}{|c|c|c|}
\hline 583 & $\begin{array}{l}\text { Tulldahl, J.,Pettersson, K.,Andersson, S. W.,Hulthen, L. Mode of infant feeding and achieved growth in adolescence: early feeding } \\
\text { patterns in relation to growth and body composition in adolescence. Obes Res.1999;7:431-7. }\end{array}$ & Design \\
\hline 584 & $\begin{array}{l}\text { Umer, A.,Hamilton, C.,Britton, C. M.,Mullett, M. D.,John, C.,Neal, W.,Lilly, C. L. Association between Breastfeeding and Childhood } \\
\text { Obesity: Analysis of a Linked Longitudinal Study of Rural Appalachian Fifth-Grade Children. Child Obes.2015;11:449-55. }\end{array}$ & IV \\
\hline 585 & $\begin{array}{l}\text { Unni, J. C., Richard, J. Growth and morbidity of breast-fed and artificially-fed infants in urban south Indian families. J Trop } \\
\text { Pediatr.1988;34:179-81. }\end{array}$ & IV \\
\hline 586 & $\begin{array}{l}\text { Vail, B.,Prentice, P.,Dunger, D. B.,Hughes, I. A.,Acerini, C. L.,Ong, K. K. Age at Weaning and Infant Growth: Primary Analysis and } \\
\text { Systematic Review. Journal of Pediatrics.2015;167:317-324.e1. }\end{array}$ & Design \\
\hline 587 & Valman, H. B. The first year of life: feeding and feeding problems. Br Med J.1980;280:457-60. & Design \\
\hline 588 & $\begin{array}{l}\text { van der Willik, E. M.,Vrijkotte, T. G.,Altenburg, T. M.,Gademan, M. G.,Kist-van Holthe, J. Exclusively breastfed overweight infants } \\
\text { are at the same risk of childhood overweight as formula fed overweight infants. Arch Dis Child.2015;100:932-7. }\end{array}$ & IV \\
\hline 589 & $\begin{array}{l}\text { van Dijk, C. E.,Innis, S. M. Growth-curve standards and the assessment of early excess weight gain in infancy. } \\
\text { Pediatrics.2009;123:102-8. }\end{array}$ & IV, DV \\
\hline 590 & $\begin{array}{l}\text { van Eijsden, M.,Meijers, C. M.,Jansen, J. E.,de Kroon, M. L.,Vrijkotte, T. G. Cultural variation in early feeding pattern and maternal } \\
\text { perceptions of infant growth. Br J Nutr.2015;114:481-8. }\end{array}$ & DV \\
\hline 591 & $\begin{array}{l}\text { van Rheenen,P. F.,de Moor,L. T.,Eschbach,S.,Brabin,B. J. A cohort study of haemoglobin and zinc protoporphyrin levels in term } \\
\text { Zambian infants: effects of iron stores at birth, complementary food and placental malaria. Eur J Clin Nutr.2008;62:1379-87. }\end{array}$ & Country \\
\hline 592 & $\begin{array}{l}\text { van } t \text { Hof Msc, M. A. The influence of breastfeeding and complementary foods on growth until three years of age in the Euro-Growth } \\
\text { Study. Pediatrics.2000;106:1281a-1281. }\end{array}$ & Design \\
\hline 593 & $\begin{array}{l}\text { Vazir,S.,Engle,P.,Balakrishna,N.,Griffiths,P. L.,Johnson,S. L.,Creed-Kanashiro,H.,Fernandez Rao,S.,Shroff,M. R.,Bentley,M. E. } \\
\text { Cluster-randomized trial on complementary and responsive feeding education to caregivers found improved dietary intake, growth } \\
\text { and development among rural Indian toddlers. Matern Child Nutr.2013;9:99-117. }\end{array}$ & IV \\
\hline 594 & $\begin{array}{l}\text { Veena SR,Krishnaveni GV,Srinivasan K,Wills AK,Hill JC,Kurpad AV,Muthayya S,Karat SC,Nalinakshi M,Fall CH. Infant feeding } \\
\text { practice and childhood cognitive performance in South India. Arch Dis Child.2010;95:347-54. }\end{array}$ & Country \\
\hline 595 & $\begin{array}{l}\text { Veena, S. R.,Krishnaveni, G. V.,Wills, A. K.,Hill, J. C.,Karat, S. C.,Fall, C. H. Glucose tolerance and insulin resistance in Indian } \\
\text { children: relationship to infant feeding pattern. Diabetologia.2011;54:2533-7. }\end{array}$ & Country \\
\hline 596 & $\begin{array}{l}\text { Vehapoglu, A.,Yazici, M.,Demir, A. D.,Turkmen, S.,Nursoy, M.,Ozkaya, E. Early infant feeding practice and childhood obesity: the } \\
\text { relation of breast-feeding and timing of solid food introduction with childhood obesity. J Pediatr Endocrinol Metab.2014;27:1181-7. }\end{array}$ & Design \\
\hline 597 & $\begin{array}{l}\text { Venancio, S. I.,Saldiva, S. R.,Mondini, L.,Levy, R. B.,Escuder, M. M. Early interruption of exclusive breastfeeding and associated } \\
\text { factors, state of Sao Paulo, Brazil. J Hum Lact.2008;24:168-74. }\end{array}$ & $\begin{array}{l}\text { Design, } \\
\text { DV }\end{array}$ \\
\hline 598 & $\begin{array}{l}\text { Verd S,Barriuso L,Gich I,Gutierrez A,Nadal-Amat J,Carreras E. Risk of early breastfeeding cessation among symmetrical, small for } \\
\text { gestational age infants. Ann Hum Biol.2013;40:146-51. }\end{array}$ & $\begin{array}{l}\text { Health } \\
\text { status, DV }\end{array}$ \\
\hline 599 & $\begin{array}{l}\text { Victora, C. G.,Matijasevich, A.,Santos, I. S.,Barros, A. J.,Horta, B. L.,Barros, F. C. Breastfeeding and feeding patterns in three birth } \\
\text { cohorts in Southern Brazil: trends and differentials. Cad Saude Publica.2008;24 Suppl 3:S409-16. }\end{array}$ & IV, DV \\
\hline 600 & $\begin{array}{l}\text { Victora,C. G.,Vaughan,J. P.,Martines,J. C.,Barcelos,L. B. Is prolonged breast-feeding associated with malnutrition?. Am J Clin } \\
\text { Nutr.1984;39:307-14. }\end{array}$ & IV \\
\hline
\end{tabular}




\begin{tabular}{|c|c|c|}
\hline 601 & Villalpando, S. Feeding mode, infections, and anthropometric status in early childhood. Pediatrics.2000;106:1282-3. & Design \\
\hline 602 & $\begin{array}{l}\text { Virtanen, S. M.,Laara, E.,Hypponen, E.,Reijonen, H.,Rasanen, L.,Aro, A.,Knip, M.,llonen, J.,Akerblom, H. K. Cow's milk } \\
\text { consumption, HLA-DQB1 genotype, and type } 1 \text { diabetes: a nested case-control study of siblings of children with diabetes. Childhood } \\
\text { diabetes in Finland study group. Diabetes.2000;49:912-7. }\end{array}$ & DV \\
\hline 603 & $\begin{array}{l}\text { Virtanen, S. M.,Rasanen, L.,Ylonen, K.,Aro, A.,Clayton, D.,Langholz, B.,Pitkaniemi, J.,Savilahti, E.,Lounamaa, R., Tuomilehto, J.,et } \\
\text { al.,. Early introduction of dairy products associated with increased risk of IDDM in Finnish children. The Childhood in Diabetes in } \\
\text { Finland Study Group. Diabetes.1993;42:1786-90. }\end{array}$ & DV \\
\hline 604 & $\begin{array}{l}\text { Vobecky,J. S.,Vobecky,J.,Shapcott,D.,Demers,P. P. Nutrient intake patterns and nutritional status with regard to relative weight in } \\
\text { early infancy. Am J Clin Nutr.1983;38:730-8. }\end{array}$ & IV \\
\hline 605 & $\begin{array}{l}\text { Wandel, M.,Fagerli, R. Aa,Olsen, P. T.,Borch-lohnsen, B.,Ek, J. Iron status and weaning practices among Norwegian and immigrant } \\
\text { infants. Nutrition Research.1996;16:251-265. }\end{array}$ & Design \\
\hline 606 & $\begin{array}{l}\text { Wang RJ,Trehan I,LaGrone LN,Weisz AJ,Thakwalakwa CM,Maleta KM,Manary MJ. Investigation of food acceptability and feeding } \\
\text { practices for lipid nutrient supplements and blended flours used to treat moderate malnutrition. J Nutr Educ Behav.2013;45:258-63. }\end{array}$ & $\begin{array}{l}\text { Health } \\
\text { status, } \\
\text { Country }\end{array}$ \\
\hline 607 & $\begin{array}{l}\text { Watt, R. G.,Tull, K. I.,Hardy, R.,Wiggins, M.,Kelly, Y.,Molloy, B.,Dowler, E.,Apps, J.,McGlone, P. Effectiveness of a social support } \\
\text { intervention on infant feeding practices: randomised controlled trial. J Epidemiol Community Health.2009;63:156-62. }\end{array}$ & IV, DV \\
\hline 608 & $\begin{array}{l}\text { Weber, M.,Grote, V.,Closa-Monasterolo, R.,Escribano, J.,Langhendries, J. P.,Dain, E.,Giovannini, M.,Verduci, E.,Gruszfeld, } \\
\text { D.,Socha, P.,Koletzko, B. Lower protein content in infant formula reduces BMl and obesity risk at school age: follow-up of a } \\
\text { randomized trial. Am J Clin Nutr.2014;99:1041-51. }\end{array}$ & IV \\
\hline 609 & $\begin{array}{l}\text { Weijs, P. J.,Kool, L. M.,van Baar, N. M.,van der Zee, S. C. High beverage sugar as well as high animal protein intake at infancy may } \\
\text { increase overweight risk at } 8 \text { years: a prospective longitudinal pilot study. Nutr J.2011;10:95. }\end{array}$ & IV \\
\hline 610 & $\begin{array}{l}\text { Wen, L. M.,Baur, L. A.,Simpson, J. M.,Xu, H.,Hayes, A. J.,Hardy, L. L., Williams, M.,Rissel, C. Sustainability of Effects of an Early } \\
\text { Childhood Obesity Prevention Trial Over Time: A Further 3-Year Follow-up of the Healthy Beginnings Trial. JAMA } \\
\text { Pediatr.2015;169:543-51. }\end{array}$ & IV \\
\hline 611 & $\begin{array}{l}\text { West, C. E.,Hernell, O.,Andersson, Y.,Sjostedt, M.,Hammarstrom, M. L. Probiotic effects on T-cell maturation in infants during } \\
\text { weaning. Clin Exp Allergy.2012;42:540-9. }\end{array}$ & IV, DV \\
\hline 612 & Westphal, R.,Phillips, G.,Irwig, L. M. Infant care and feeding in an urban black population. S Afr Med J.1981;60:778-81. & DV, Date \\
\hline 613 & $\begin{array}{l}\text { Weyermann, M., Rothenbacher, D.,Brenner, H. Duration of breastfeeding and risk of overweight in childhood: a prospective birth } \\
\text { cohort study from Germany. Int J Obes (Lond).2006;30:1281-7. }\end{array}$ & IV \\
\hline 614 & $\begin{array}{l}\text { Wharf,S. G.,Fox,T. E.,Fairweather-Tait,S. J.,Cook,J. D. Factors affecting iron stores in infants 4-18 months of age. Eur J Clin } \\
\text { Nutr.1997;51:504-9. }\end{array}$ & Design \\
\hline 615 & $\begin{array}{l}\text { Whitehead, R. G.,Paul, A. A.,Ahmed, E. A. Weaning practices in the United Kingdom and variations in anthropometric development. } \\
\text { Acta Paediatr Scand Suppl.1986;323:14-23. }\end{array}$ & Design, IV \\
\hline 616 & Whitehead, R. G.,Paul, A. A. Infant growth and human milk requirements. A fresh approach. Lancet.1981;2:161-3. & IV \\
\hline 617 & Whitten, C. F.,Stewart, R. A. The effect of dietary sodium in infancy on blood pressure and related factors. Studies of infants fed & IV \\
\hline
\end{tabular}




\begin{tabular}{|c|c|c|}
\hline & onths at eight months and eight years of age. Acta Paediatr Sca & \\
\hline 618 & $\begin{array}{l}\text { Wiberger, M.,Eiben, G.,Lissner, L.,Mehlig, K.,Papoutsou, S.,Hunsberger, M. Children consuming milk cereal drink are at increased } \\
\text { risk for overweight: The IDEFICS Sweden study, on behalf of the IDEFICS Consortium. Scand J Public Health.2014;42:518-24. }\end{array}$ & Design \\
\hline 619 & $\begin{array}{l}\text { Wigg, N. R.,Tong, S.,McMichael, A. J.,Baghurst, P. A.,Vimpani, G., Roberts, R. Does breastfeeding at six months predict cognitive } \\
\text { development?. Aust N Z J Public Health.1998;22:232-6. }\end{array}$ & IV \\
\hline 620 & $\begin{array}{l}\text { Wijga,A.,Vyas,U.,Vyas,A.,Sharma,V.,Pandya,N.,Nabarro,D. Feeding, illness and nutritional status of young children in rural Gujarat. } \\
\text { Hum Nutr Clin Nutr.1983;37:255-69. }\end{array}$ & Design, IV \\
\hline 621 & $\begin{array}{l}\text { Williams, D. M.,Martin, R. M.,Davey Smith, G.,Alberti, K. G.,Ben-Shlomo, Y.,McCarthy, A. Associations of infant nutrition with insulin } \\
\text { resistance measures in early adulthood: evidence from the Barry-Caerphilly Growth (BCG) study. PLoS One.2012;7:e34161. }\end{array}$ & IV, DV \\
\hline 622 & $\begin{array}{l}\text { Williams, J.,Wolff, A.,Daly, A.,MacDonald, A.,Aukett, A.,Booth, I. W. Iron supplemented formula milk related to reduction in } \\
\text { psychomotor decline in infants from inner city areas: randomised study. Bmj.1999;318:693-7. }\end{array}$ & IV \\
\hline 623 & Winick, M. The role of early nutrition in subsequent development and optimal future health. Bull N Y Acad Med.1989;65:1020-5. & Design \\
\hline 624 & Winkelstein,M. L. Overfeeding in infancy: the early introduction of solid foods. Pediatr Nurs.1984;10:205-8, 236. & Design \\
\hline 625 & $\begin{array}{l}\text { Wölfle, J. Growth and puberty in German children: is there still a positive secular trend? In reply..Consumption of milk as a vital } \\
\text { factor in growth development. Melnik B, Dtsch Arzetebl 2009, volume 206. Deutsches Aerzteblatt International.2009;106:656-656. }\end{array}$ & Design \\
\hline 626 & $\begin{array}{l}\text { Wright, C. M.,Parkinson, K. N.,Drewett, R. F. Why are babies weaned early? Data from a prospective population based cohort study. } \\
\text { Arch Dis Child.2004;89:813-6. }\end{array}$ & DV \\
\hline 627 & $\begin{array}{l}\text { Wright, C. M.,Parkinson, K.,Scott, J. Breast-feeding in a UK urban context: who breast-feeds, for how long and does it matter?. } \\
\text { Public Health Nutr.2006;9:686-91. }\end{array}$ & IV \\
\hline 628 & $\begin{array}{l}\text { Wright, M. J.,Bentley, M. E.,Mendez, M. A.,Adair, L. S. The interactive association of dietary diversity scores and breast-feeding } \\
\text { status with weight and length in Filipino infants aged 6-24 months. Public Health Nutr.2015;18:1762-73. }\end{array}$ & Country \\
\hline 629 & $\begin{array}{l}\text { Yew, K. S.,Webber, B.,Hodges, J.,Carter, N. J. Clinical inquiries: are there any known health risks to early introduction of solids to an } \\
\text { infant's diet?. J Fam Pract.2009;58:219-20. }\end{array}$ & Design \\
\hline 630 & $\begin{array}{l}\text { Young RJ,Antonson DL,Ferguson PW,Murray ND,Merkel K,Moore TE. Neonatal and infant feeding: effect on bone density at } 4 \\
\text { years. J Pediatr Gastroenterol Nutr.2005;41:88-93. }\end{array}$ & Design \\
\hline 631 & $\begin{array}{l}\text { Yousafzai, A. K., Rasheed, M. A.,Rizvi, A.,Armstrong, R.,Bhutta, Z. A. Effect of integrated responsive stimulation and nutrition } \\
\text { interventions in the Lady Health Worker programme in Pakistan on child development, growth, and health outcomes: a cluster- } \\
\text { randomised factorial effectiveness trial. Lancet.2014;384:1282-93. }\end{array}$ & Country \\
\hline 632 & $\begin{array}{l}\text { Ystrom, E. Breastfeeding cessation and symptoms of anxiety and depression: a longitudinal cohort study. BMC Pregnancy } \\
\text { Childbirth.2012;12:36. }\end{array}$ & IV, DV \\
\hline 633 & $\begin{array}{l}\text { Zadik Z,Borondukov E,Zung A,Reifen R. Adult height and weight of breast-fed and bottle-fed Israeli infants. J Pediatr Gastroenterol } \\
\text { Nutr.2003;37:462-7. }\end{array}$ & IV \\
\hline 634 & $\begin{array}{l}\text { Zaman, S.,Jalil, F.,Saleemi, M. A.,Mellander, L.,Ashraf, R. N.,Hanson, L. A. Changes in feeding patterns affect growth in children 0- } \\
24 \text { months of age living in socioeconomically different areas of Lahore, Pakistan. Adv Exp Med Biol.2002;503:49-56. }\end{array}$ & Country \\
\hline
\end{tabular}




\begin{tabular}{|l|l|l|}
\hline 635 & $\begin{array}{l}\text { Zaman,S.,Ashraf,R. N.,Martines,J. Training in complementary feeding counselling of healthcare workers and its influence on } \\
\text { maternal behaviours and child growth: a cluster-randomized controlled trial in Lahore, Pakistan. J Health Popul Nutr.2008;26:210- } \\
22 .\end{array}$ & $\begin{array}{l}\text { IV, } \\
\text { Country }\end{array}$ \\
\hline 636 & $\begin{array}{l}\text { Zavaleta,N.,Kvistgaard,A. S.,Graverholt,G.,Respicio,G.,Guija,H.,Valencia,N.,Lonnerdal,B. Efficacy of an MFGM-enriched } \\
\text { complementary food in diarrhea, anemia, and micronutrient status in infants. J Pediatr Gastroenterol Nutr.2011;53:561-8. }\end{array}$ & DV \\
\hline 637 & $\begin{array}{l}\text { Zhang,J.,Shi,L.,Chen,D. F.,Wang,J.,Wang,Y. Effectiveness of an educational intervention to improve child feeding practices and } \\
\text { growth in rural China: updated results at 18 months of age. Matern Child Nutr.2013;9:118-29. }\end{array}$ & IV \\
\hline 638 & $\begin{array}{l}\text { Zhu, B.,Zhang, J.,Qiu, L.,Binns, C.,Shao, J.,Zhao, Y.,Zhao, Z. Breastfeeding Rates and Growth Charts--the Zhejiang Infant Feeding } \\
\text { Trial. Int J Environ Res Public Health.2015;12:7337-47. }\end{array}$ & IV, DV \\
\hline 639 & $\begin{array}{l}\text { Ziegler, E. E.,Fields, D. A.,Chernausek, S. D.,Steenhout, P.,Grathwohl, D.,Jeter, J. M.,Nelson, S. E.,Haschke, F. Adequacy of Infant } \\
\text { Formula With Protein Content of 1.6 g/100 kcal for Infants Between 3 and 12 Months. J Pediatr Gastroenterol Nutr.2015;61:596- } \\
\text { 603. }\end{array}$ & IV \\
\hline 640 & $\begin{array}{l}\text { Ziegler,E. E.,Fomon,S. J.,Nelson,S. E.,Rebouche,C. J.,Edwards,B. B.,Rogers,R. R.,Lehman,L. J. Cow milk feeding in infancy: } \\
\text { further observations on blood loss from the gastrointestinal tract. J Pediatr.1990;116:11-8. }\end{array}$ & IV, DV \\
\hline 641 & $\begin{array}{l}\text { Zive, M. M.,McKay, H.,Frank-Spohrer, G. C.,Broyles, S. L.,Nelson, J. A.,Nader, P. R. Infant-feeding practices and adiposity in 4-y- } \\
\text { old Anglo- and Mexican-Americans. Am J Clin Nutr.1992;55:1104-8. }\end{array}$ & Design \\
\hline 642 & $\begin{array}{l}\text { Zutavern, A.,Brockow, I.,Schaaf, B.,von Berg, A.,Diez, U.,Borte, M.,Kraemer, U.,Herbarth, O.,Behrendt, H.,Wichmann, H. } \\
\text { E.,Heinrich, J. Timing of solid food introduction in relation to eczema, asthma, allergic rhinitis, and food and inhalant sensitization at } \\
\text { the age of 6 years: results from the prospective birth cohort study LISA. Pediatrics.2008;121:e44-52. }\end{array}$ & DV \\
\hline
\end{tabular}

${ }_{1}^{1}$ Abbreviations: DV- Dependent variable; IV- Independent variable/exposure/intervention 


\section{APPENDIX}

The table below describes study and sample characteristics from the included articles, organized by study design then alphabetical order of the first author's last name.

\section{Supplemental Table S4. Description of the evidence examining timing of introduction of CFB and growth, size, and body composition.}

\section{Randomized controlled trials}

Bainbridge, 1996

Randomized Controlled Trial;

U.S.

Analytic N: 41

Attrition: 0\%

Power Analysis/Sample Size

Calculation: NR

Sex:

$\sim 49 \%$ Female

Race/Ethnicity:

73.2\% White, $26.8 \%$ Black

Background Diet:

All FF with same formula from birth to $16 \mathrm{wk}$

\section{Sample Size:}

Baseline N: 41

\section{Intervention/Exposure:}

Age of CFB introduction: CFB at 16wk vs. EFF until 26wk CFB: Rice cereal from 16-26wk; Both groups were FF and the EFF group continued exclusive use of formula from 16-26wk

Age: $4 \mathrm{mo}$

Assessment Methods:

Food record, 3d

Outcomes:

Weight; Length; Body composition (Adiposity)

Age: 16wk, 26wk, 16-26wk

Assessment Methods:

Body composition: Skinfold thickness: calipers; abdominal, MUAC, triceps, mid-thigh, chest, subscapular, suprailiac

Weight, length: NR, digital scale and length board

$\mathrm{HC}: \mathrm{NR}$

\section{Confounders accounted for:}

\section{$\mathrm{N} / \mathrm{A}$}

Other: At baseline, reported NSD in sex, race/ethnicity, birth weight, gestational age; and all were FF

\section{Jonsdottir, 2012}

Randomized Controlled Trial; Iceland

\section{Sample Size:}

Baseline N: 119

Analytic N: 100

\section{Intervention/Exposure:}

EBF infants were randomized to one of 2 groups:

EBF: Remained exclusively BF until $6 \mathrm{mo}$

CFB: Receive CFB starting at age $4 \mathrm{mo}$

CFB: Foods other than BM; Diaries indicated every new food added to CFB infant diet from $4 \mathrm{mo}$ to $6 \mathrm{mo}$

\section{Limitations:}

Cannot determine whether groups were similar at baseline on education, SES, or maternal age, early CFB group was $1 \mathrm{~cm}$ shorter than EBF group at baseline; Participants (infants/mothers) were not blinded; Cannot determine if investigators were blinded

For technical reasons due to sample size limitations, outcome measurements were available for $n: 23$ in group receiving CFB at 16 wk group and $n: 18$ in the group delaying CFB to 26wk

Sample size was very small

\section{Limitations}

Cannot determine if investigators or outcome assessors were blinded; Participants were not blinded;

Outcome assessment methods were not validi/reliable (non-duplicate and 


\begin{tabular}{|c|c|c|}
\hline $\begin{array}{l}\text { Attrition: } 16 \% \\
\text { Sample Size Calculation: } \mathrm{N}=50 \\
\text { (to detect differences in Fe } \\
\text { status) } \\
\text { Sex: } \\
55 \% \text { Female } \\
\text { Race/Ethnicity: } \\
\text { NR } \\
\text { Background Diet: } \\
\text { All infants were EBF up to } 4 \mathrm{mo} \text {, } \\
\text { and continue to be BF through } \\
6 \text { mo }\end{array}$ & $\begin{array}{l}\text { Age: } 4 \text { mo } \\
\text { Assessment Methods: } \\
\text { Maternal diet diary; Food record, 3d, weighed } \\
\text { Outcomes: } \\
\text { Weigh; Length; HC } \\
\text { Age: birth-6mo; 4-6mo } \\
\text { Assessment Methods: } \\
\text { Weight: Seca scale; used for WAZ by WHO standards } \\
\text { Length: measuring board; used for LAZ by WHO standards } \\
\text { HC: non-stretchable tape } \\
\text { Confounders accounted for: } \\
\text { Feeding practices: All EBF } \\
\text { Gestational age: All full-term } \\
\text { Other: Delivery mode }\end{array}$ & $\begin{array}{l}\text { various assessors; data collected in } \\
\text { routine health care visits at health } \\
\text { centers) }\end{array}$ \\
\hline $\begin{array}{l}\text { Jonsdottir, 2013 } \\
\text { Randomized Controlled Trial; } \\
\text { Iceland } \\
\text { Sample Size: } \\
\text { Baseline } \mathrm{N}=119 \text { ( } \mathrm{N}=100 \\
\text { completed the intervention) } \\
\text { Analytic } \mathrm{N}=54-78 \\
\text { Attrition }=34.5 \% \\
\text { Power Analysis and Sufficient } \\
\text { Sample Size: } \mathrm{N}=66 \text { (to detect } \\
\text { an } 11.2 \text { point difference in the } \\
\text { Brigance screening test at } 30- \\
35 \text { mo, } 80 \% \text { power) } \\
\text { Sex: } \\
54.5 \% \text { Female } \\
\text { Race/Ethnicity: } \\
\text { NR } \\
\text { Background Diet: } \\
\text { In lceland, } 50 \% \text { of infants are }\end{array}$ & $\begin{array}{l}\text { Intervention/Exposure: } \\
\text { EBF infants were randomized to one of } 2 \text { groups: } \\
\text { EBF: Remained exclusively BF until } 6 \mathrm{mo} \\
\text { CFB: Receive CFB starting at age } 4 \mathrm{mo} \\
\text { CFB: Foods other than BM; Diaries indicated every new food added } \\
\text { to CFB infant diet from } 4 \mathrm{mo} \text { to } 6 \mathrm{mo} \\
\text { Age: } 4 \mathrm{mo} \\
\text { Assessment Methods: } \\
\text { Maternal diet diary; Food record, } 3 \mathrm{~d} \text {, weighed } \\
\text { Outcomes: } \\
\text { HC } \\
\text { Age: birth-18mo } \\
\text { Assessment Methods: } \\
\text { HC: gain; measured with non-stretchable tape (Jonsdottir, 2012) } \\
\text { Confounders accounted for: } \\
\text { Feeding practices: All EBF } \\
\text { Gestational age: All full-term } \\
\text { Other: Delivery mode }\end{array}$ & $\begin{array}{l}\text { Limitations: } \\
\text { Cannot determine if investigators or } \\
\text { outcome assessors were blinded; } \\
\text { Participants were not blinded; Cannot } \\
\text { determine validity/reliability of outcome } \\
\text { measurements (non-duplicate and } \\
\text { various assessors; lack of detail for } \\
\text { HC; data collected in routine health } \\
\text { care visits at health centers) } \\
\text { Authors note that the analysis my have } \\
\text { been underpowered (small sample } \\
\text { size) to detect potential small } \\
\text { differences in developmental outcomes }\end{array}$ \\
\hline
\end{tabular}




\begin{tabular}{|c|c|c|}
\hline $\begin{array}{l}\text { EBF through } 4 \mathrm{mo} \text { and } 35 \% \\
\text { through } 5 \mathrm{mo}\end{array}$ & & \\
\hline $\begin{array}{l}\text { Jonsdottir, } 2014 \\
\text { Randomized Controlled Trial; } \\
\text { Iceland } \\
\text { Sample Size: } \\
\text { Baseline N: } 119 \\
\text { Analytic N: } 100 \\
\text { Attrition: } 16 \% \\
\text { Sample Size Calculation: N=50 } \\
\text { (to detect differences in Fe } \\
\text { status) } \\
\text { Sex: } \\
54.5 \% \text { Female } \\
\text { Race/Ethnicity: } \\
\text { NR } \\
\text { Background Diet: } \\
\text { In Iceland, } 50 \% \text { of infants are } \\
\text { EBF through 4mo and } 35 \% \\
\text { through } 5 \mathrm{mo}\end{array}$ & $\begin{array}{l}\text { Intervention/Exposure: } \\
\text { EBF infants were randomized to one of } 2 \text { groups: } \\
\text { EBF: Remained exclusively BF until } 6 \text { mo } \\
\text { CFB: Receive CFB starting at age } 4 \text { mo } \\
\text { CFB: Foods other than BM; Diaries indicated every new food added } \\
\text { to CFB infant diet from } 4 \text { mo to } 6 \text { mo } \\
\text { Age: } 4 \text { mo } \\
\text { Assessment Methods: } \\
\text { Maternal diet diary; Food record, 3d, weighed } \\
\text { Outcomes: } \\
\text { Weight status; Weight; Length; HC } \\
\text { Age: } 18,29-38 \text { mo } \\
\text { Assessment Methods: } \\
\text { Weight status: risk for overweight classified as }>1 S D \text { above BMI-for- } \\
\text { age (WHO); Overweight classified as }>2 S D \text { above; Obesity } \\
\text { classified as >3SD } \\
\text { Weight: via Seca scale; WAZ and } \Delta \text { WAZ by WHO standards } \\
\text { Length: via measuring board/body meter; LAZ and } \Delta \text { LAZ by WHO } \\
\text { standards } \\
\text { HC: non-stretchable tape } \\
\text { Confounders accounted for: } \\
\text { Feeding practices: All EBF } \\
\text { Gestational age: All full-term } \\
\text { Other: Delivery mode }\end{array}$ & $\begin{array}{l}\text { Limitations: } \\
\text { Groups differed in mode of delivery, } \\
\text { which was not adjusted for in analyses } \\
\text { Cannot determine if investigators or } \\
\text { outcome assessors were blinded; } \\
\text { Participants were not blinded; Cannot } \\
\text { determine validity/reliability of outcome } \\
\text { measurements (non-duplicate and } \\
\text { various assessors; data collected in } \\
\text { routine health care visits at health } \\
\text { centers) } \\
\text { Relatively small sample-size }\end{array}$ \\
\hline $\begin{array}{l}\text { Wells, } 2012 \\
\text { Randomized Controlled Trial; } \\
\text { Iceland } \\
\text { Sample Size: } \\
\text { CFB Group Baseline N: } 61 \\
\text { EBF Group Baseline N: } 58\end{array}$ & $\begin{array}{l}\text { Intervention/Exposure: } \\
\text { CFB Group: Advised to introduce CFB at } 4 \mathrm{mo} \text { (no specific advice } \\
\text { regarding type/amount of CFB to introduce) } \\
\text { EBF Group: Advised to exclusively BF through } 6 \mathrm{mo} \\
\text { Age: } 4-6 \mathrm{mo} \\
\text { Assessment Methods: } \\
\text { Food records, } 3 \mathrm{~d}\end{array}$ & $\begin{array}{l}\text { Limitations: } \\
\text { Groups differed at baseline on } \\
\text { multiparity, vaginal delivery; cannot } \\
\text { determine whether these differences } \\
\text { were adjusted for in subsequent } \\
\text { analyses; Participants, outcome } \\
\text { assessors were not blinded; cannot } \\
\text { determine whether investigators were }\end{array}$ \\
\hline
\end{tabular}




\begin{tabular}{|c|c|c|}
\hline $\begin{array}{l}\text { CFB Group Analytic N: } 50 \\
\text { EBF Group Analytic N: } 50 \\
\text { Attrition: } 16 \% \\
\text { Power Analysis and Sufficient } \\
\text { Sample Size: } \mathrm{N}=50 / \mathrm{group} \text { to } \\
\text { detect differences of } 75 \mathrm{~g} / \mathrm{d} \\
\text { with } 80 \% \text { power and } 5 \% \\
\text { significance } \\
\text { Sex: } \\
50 \% \text { Female } \\
\text { Race/Ethnicity: } \\
\text { NR } \\
\text { Background Diet: } \\
\text { EBF: } 100 \% \text { at baseline }\end{array}$ & $\begin{array}{l}\text { Outcomes: } \\
\text { Body composition (Adiposity; BMI); Weight; Length, } \mathrm{HCz} \\
\text { Age: } 6 \text { mo } \\
\text { Assessment Methods: } \\
\text { Body composition: } \\
\text { - Lean/fat mass: Calculated based on total body water } \\
\text { - BMI: Calculated using measured weight, length } \\
\text { Weight: Measured by study personnel } \\
\text { Length: Measured by study personnel, recumbent } \\
\text { HC: } z-\text { score, measured by study personnel } \\
\text { Confounders accounted for: } \\
\text { None }\end{array}$ & $\begin{array}{l}\text { blinded; Cannot determine the } \\
\text { validity/reliability of outcome } \\
\text { assessment (i.e., single vs multiple } \\
\text { measures); Adequacy of statistical } \\
\text { methods insufficient (no description of } \\
\text { which potential confounders were } \\
\text { included in analyses) } \\
\text { Data from the RCT in Jonsdottir, 2012; } \\
\text { Jonsdottir, 2013; Jonsdottir, } 2014\end{array}$ \\
\hline \multicolumn{3}{|l|}{ Prospective cohort studies } \\
\hline $\begin{array}{l}\text { Abraham, } 2012 \\
\text { Prospective Cohort Study; } \\
\text { Scotland } \\
\text { Sample Size: } \\
\text { Baseline N: } 5,217 \\
\text { Analytic N: 4,493 (completed } \\
\text { survey at 9-12mo); 3,994 } \\
\text { (completed outcomes at 45- } \\
\text { 48mo) } \\
\text { Attrition: 13.9\% } \\
\text { Power Analysis: NR } \\
\text { Sex: } \\
\text { NR } \\
\text { Race/Ethnicity: } \\
4 \% \text { non-white ethnic group } \\
\text { (Bradshaw, 2007) } \\
\text { Background Diet: } \\
61.0 \% \text { ever BF, 39.0\% never } \\
\text { BF }\end{array}$ & $\begin{array}{l}\text { Intervention/Exposure: } \\
\text { Age of CFB introduction: } 0-3 \text { vs. } 4-5 ; 0-3 \text { vs. } 6-10 \mathrm{mo} \\
\text { Age: } 0-24 \mathrm{mo} \\
\text { Assessment Methods: } \\
\text { Caregiver self-report at } 9-12 \mathrm{mo} \\
\text { Outcomes: } \\
\text { Weight status (Overweight/obesity) } \\
\text { Age: } 45-48 m o \\
\text { Assessment Methods: } \\
\text { Weight status: risk of overweight/obese BMI >/=1.04, or not } \\
\text { overweight/obese</=1.04 (UK } 1990 \text { ref. curves BMIZ cut-off) } \\
\text { Weight, height: NR- } \\
\text { Confounders accounted for: } \\
\text { SES: } X \\
\text { Birth size: } X \text { (Birth weight) }\end{array}$ & $\begin{array}{l}\text { Limitations: } \\
\text { Cannot determine whether groups } \\
\text { were similar at baseline on key } \\
\text { characteristics; Key confounders from } \\
\text { the analytic framework were NR or } \\
\text { adjusted for in analyses including sex, } \\
\text { race/ethnicity, maternal age, or } \\
\text { gestational age; Cannot determine if } \\
\text { outcome assessors were blinded, } \\
\text { primary interviewers were unblinded } \\
\text { Other: IV/Exposure was self-report; } \\
\text { Bradshaw, } 2007 \text { summarizes Sweep } 1 \\
\text { baseline characteristics } \\
\text { - }\end{array}$ \\
\hline
\end{tabular}




\begin{tabular}{|c|c|c|}
\hline $\begin{array}{l}\text { Agras, } 1990 \\
\text { Prospective Cohort Study; U.S. } \\
\text { Sample Size: } \\
\text { Baseline N: } 99 \\
\text { Analytic N: } 54 \\
\text { Attrition: } 45 \% \\
\text { Power Analysis/Sample Size } \\
\text { Calculation: NR } \\
\text { Sex: } \\
\text { NR } \\
\text { Race/Ethnicity: } \\
\text { NR } \\
\text { Background Diet: } \\
\text { BF and/or FF }\end{array}$ & $\begin{array}{l}\text { Intervention/Exposure: } \\
\text { Age of CFB introduction: Weeks, continuous } \\
\text { Age: } 2,4,12,20 \text { wk } \\
\text { Assessment Methods: } \\
\text { Maternal report } \\
\text { Outcomes: } \\
\text { BMI; skinfold thickness } \\
\text { Age: } 1,2,3 \text { a/o 6y } \\
\text { Assessment Methods: } \\
\text { Body composition: skin-fold thickness, and log BMI (more normally } \\
\text { distributed) calculated from measures } \\
\text { Weight, height: measured in the laboratory } \\
\text { Confounders accounted for: } \\
\text { Education: } X \\
\text { Sex: } X \\
\text { Feeding practices: } X \\
\text { Birth size: } X \text { (Birth BMI) } \\
\text { Other: parent BMl, \# feedings/d; sucking pressure, interval of } \\
\text { sucking bursts, caloric intake during suckling, active feeding time }\end{array}$ & $\begin{array}{l}\text { Limitations: } \\
\text { Cannot determine whether groups } \\
\text { were similar at baseline on key } \\
\text { characteristics; Cannot determine } \\
\text { whether outcome assessors were } \\
\text { blinded; Due to limited information } \\
\text { provided about methods,; Did not } \\
\text { adjust for potential key confounders } \\
\text { (SES, maternal age, race/ethnicity, } \\
\text { gestational age); Did not account for } \\
\text { high attrition rate ( } 45 \%) \\
\text { Log birth BMI was used as birth size } \\
\text { variable in regression; Only assessed } \\
\text { infant feeding practices through } 5 \text { mo of } \\
\text { age_ } \\
\text { Cannot rule out reverse causality } \\
\text { (vigorous BF may influence timing CFB } \\
\text { introduction) }\end{array}$ \\
\hline $\begin{array}{l}\text { Atkins, } 2016 \\
\text { Prospective Cohort Study; } \\
\text { Australia } \\
\text { Sample Size: } \\
\text { Baseline N: } 542 \\
\text { Analytic N: } 423 \\
\text { Attrition: } 22 \% \\
\text { Power Analysis/Sample Size } \\
\text { Calculation: NR } \\
\text { Sex: } \\
\text { 47\% Female } \\
\text { Race/Ethnicity: } \\
\text { NR } \\
\text { Background Diet: } \\
2 \% \text { never BF, } 46 \% \text { BF at } 9 \mathrm{mo}, \\
9 \% \text { BF at } 20 \text { mo }\end{array}$ & $\begin{array}{l}\text { Intervention/Exposure: } \\
\text { Age of CFB introduction: }<6 \text { vs. }>6 \mathrm{mo} \\
\text { Age: } 3 \mathrm{mo} \\
\text { Assessment Methods: } \\
3,24-\mathrm{h} \text { recalls by parent } \\
\text { Outcomes: } \\
\text { Weight; Height } \\
\text { Age: } 9 \mathrm{mo}, 20 \mathrm{mo} \\
\text { Assessment Methods: } \\
\text { Weight, length: Measured by trained staff using digital scales and } \\
\text { calibrated measuring mat } \\
\text { Confounders accounted for: } \\
\text { None }\end{array}$ & $\begin{array}{l}\text { Limitations: } \\
\text { Cannot determine whether groups } \\
\text { were similar at baseline on key } \\
\text { characteristics; Cannot determine } \\
\text { whether outcome assessors were } \\
\text { blinded; Cannot determine } \\
\text { relability/validity of outcome } \\
\text { assessment (used measuring mat, not } \\
\text { length board); Did not adjust for any } \\
\text { potential key confounders (education, } \\
\text { SES, sex, maternal age, race/ethnicity, } \\
\text { feeding practices, birth size, } \\
\text { gestational age) } \\
\text { Cannot determine if analyses adjusted } \\
\text { for differences in birth weight and }\end{array}$ \\
\hline
\end{tabular}




\begin{tabular}{|c|c|c|}
\hline & & $\begin{array}{l}\text { length differed between groups; } \\
\text { weight/length at 9mo not shown) } \\
\text { *Weight/length were secondary } \\
\text { oucomes }\end{array}$ \\
\hline $\begin{array}{l}\text { Ay, } 2008 \\
\text { Prospective Cohort Study; The } \\
\text { Netherlands } \\
\text { Sample Size: } \\
\text { Baseline N: } 1232 \\
\text { Analytic N: } 1012 \\
\text { Attrition: } 17.9 \% \\
\text { Power Analysis/Sample Size } \\
\text { Calculation: NR } \\
\text { Sex: } \\
48.2 \% \text { Female } \\
\text { Race/Ethnicity: } \\
100 \% \text { "Dutch ethnicity" } \\
\text { Background Diet: } \\
90 \% \text { ever BF, duration of BF } \\
=\sim 5 m o\end{array}$ & $\begin{array}{l}\text { Intervention/Exposure: } \\
\text { Age of CFB introduction: }<5 \text { or }>5 \mathrm{mo} \\
\text { CFB: Fruit snack } \\
\text { Age: } 2,6 \text {, and } 12 \text { mo } \\
\text { Assessment Methods: } \\
\text { Caregiver questionnaire } \\
\text { Outcomes: } \\
\text { Body composition (Adiposity) } \\
\text { Age: } 24 \text { mo } \\
\text { Assessment Methods: } \\
\text { Body composition: sum of skinfold thickness (biceps, triceps, } \\
\text { suprailiacal, subscapular) measured by study personnel using } \\
\text { calipers } \\
\text { Confounders accounted for: } \\
\text { Education: } X \\
\text { Sex: } X \\
\text { Maternal age: } X \\
\text { Gestational age: } X \\
\text { Other: Smoking }\end{array}$ & $\begin{array}{l}\text { Limitations: } \\
\text { Cannot determine whether groups } \\
\text { were similar at baseline on key } \\
\text { characteristics; Cannot determine } \\
\text { whether outcome assessors were } \\
\text { blinded; Did not adjust for any key } \\
\text { confounders (SES, } \\
\text { race/ethnicity/feeding practices, birth } \\
\text { size) }\end{array}$ \\
\hline $\begin{array}{l}\text { Baird, } 2008 \\
\text { Prospective Cohort Study; U.K. } \\
\text { Sample Size: } \\
\text { Baseline } N=1,973 \\
\text { Analytic } N=1,740 \text { at } 6 \mathrm{mo} ; \\
1335 \text { at } 12 \mathrm{mo} \\
\text { Attrition }=11.8 \% ; 32.4 \% \text { at } \\
12 \mathrm{mo} \\
\text { Power Analysis and Sufficient } \\
\text { Sample Size: NR }\end{array}$ & $\begin{array}{l}\text { Intervention/Exposure: } \\
\text { Age of CFB introduction: early }(<3,3 \text {, at } 4 \mathrm{mo}) \text { or later }(5 \mathrm{mo}+) \\
\text { CFB: Any other food aside from HM or HM substitute; } 99 \% \\
\text { introduced CFB }<5 \mathrm{mo} \\
\text { Age: } 12 \mathrm{mo} \\
\text { Assessment Methods: } \\
\text { Timing: Maternal report } \\
\text { Type: FFQ over } 7 \mathrm{~d} \text { at } 6 \mathrm{mo} \text {; FFQ over } 28 \mathrm{~d} \text { at } 12 \mathrm{mo} \text {; Dietary patterns } \\
\text { identified by PCA }\end{array}$ & $\begin{array}{l}\text { Limitations: } \\
\text { Cannot determine whether groups } \\
\text { were similar at baseline; Unclear } \\
\text { whether outcome assessors were } \\
\text { blinded to the infants' feeding histories; } \\
\text { Did not adjust for potential key } \\
\text { confounders (SES, maternal age, } \\
\text { race/ethnicity, feeding practices, birth } \\
\text { size, gestational age) }\end{array}$ \\
\hline
\end{tabular}




\begin{tabular}{|c|c|c|}
\hline $\begin{array}{l}\text { Sex: } \\
46.9 \% \text { female } \\
\text { Race/Ethnicity: } \\
94 \% \text { white } \\
\text { Background Diet: } \\
0-6 \mathrm{mo:} 7.5 \% \mathrm{BF} ; 21 \% \mathrm{FF}, \\
\sim 71.5 \% \mathrm{MF} \text { combination of BF } \\
\text { and FF assessed via caregiver- } \\
\text { report at } 6 \mathrm{mo}\end{array}$ & $\begin{array}{l}\text { Outcomes: } \\
\text { Body composition; Weight; Length } \\
\text { Age: } 6 \mathrm{mo}, 0-6 \mathrm{mo} \\
\text { Assessment Methods: } \\
\text { Weight: digital scale; crude and change in weight SD score from 0- } \\
6 \mathrm{mo} \text { and } 6-12 \mathrm{mo} \\
\text { Length: infantometer; crude and change in length SD score from 0- } \\
6 \text { mo and } 6-12 \text { mo } \\
\text { Body composition: triceps and subscapular skinfold calipers; crude } \\
\text { and thickness SD score from 0-6mo and 6-12mo } \\
\text { Confounders accounted for: } \\
\text { Education: } X \\
\text { Sex: } X \\
\text { Gestational age: } X \\
\text { Other: Parity; Smoking }\end{array}$ & $\begin{array}{l}\text { BF duration and infant diet was } \\
\text { retrospectively self-reported by } \\
\text { mothers at } 6 \text { and } 12 \mathrm{mo} \text {, thus may have } \\
\text { been biased; ethnic minorities were } \\
\text { underrepresented in this sample. }\end{array}$ \\
\hline $\begin{array}{l}\text { Baker, } 2004 \\
\text { Prospective Cohort Study; } \\
\text { Denmark } \\
\text { Sample Size: } \\
\text { Baseline } N=5,845 \\
\text { Analytic } N=3,768 \\
\text { Attrition }=36.5 \% \\
\text { Power Analysis and Sufficient } \\
\text { Sample Size: NR } \\
\text { Sex: } \\
49.5 \% \text { Female } \\
\text { Race/Ethnicity: } \\
\text { NR } \\
\text { Background Diet: } \\
\text { Duration of full BF: } ~ 16 w k \\
\text { Duration of BF: } 32 \mathrm{wk}\end{array}$ & $\begin{array}{l}\text { Intervention/Exposure: } \\
\text { Age of CFB introduction: }<16 \text { vs. }>16 w k \\
\text { CFB: Mush or porridge } \\
\text { Age: } 12 \text { mo } \\
\text { Assessment Methods: } \\
\text { Maternal report } \\
\text { Outcomes: } \\
\text { Weight } \\
\text { Age: Birth-1y } \\
\text { Assessment Methods: } \\
\text { Weight: gain birth-1y based on maternal report from the "green } \\
\text { book" health record from physicians } \\
\text { Confounders accounted for: } \\
\text { Education: } X \\
\text { SES: } X \text { (income) } \\
\text { Sex: } X \\
\text { Maternal age: } X \\
\text { Feeding practices: } X \\
\text { Birth size: } X \text { (Birth weight) }\end{array}$ & $\begin{array}{l}\text { Limitations: } \\
\text { Cannot determine whether groups } \\
\text { were similar at baseline on } \\
\text { race/ethnicity; Outcome assessors } \\
\text { were not blinded-both the independent } \\
\text { and dependent variables were } \\
\text { measured using non-validated } \\
\text { methods (maternal self-report); Did not } \\
\text { account for high loss to follow-up } \\
\text { (>35\%); Did not adjust for potenital key } \\
\text { confounder of race/ethnicity } \\
\text { Did not collect types-amounts of CFB } \\
\text { or composition, or energy expenditure, } \\
\text { or timing of introduction of formula }\end{array}$ \\
\hline
\end{tabular}




\begin{tabular}{|c|c|c|}
\hline & $\begin{array}{l}\text { Gestational age: } X \\
\text { Other: Parity; Delivery mode; Infant age; Maternal occupation; } \\
\text { Spouse; Gestational weight gain; Smoking; Infant length at 1y } \\
\text { (reverse causality) }\end{array}$ & \\
\hline $\begin{array}{l}\text { Barrera, } 2016 \\
\text { Prospective Cohort Study; } \\
\text { United States } \\
\text { Sample Size: } \\
\text { Baseline N: } 1542 \\
\text { Analytic N: } 1181 \\
\text { Attrition: 23.4\% } \\
\text { Power Analysis and Sufficient } \\
\text { Sample Size: NR } \\
\text { Sex: } \\
50.1 \% \text { Female } \\
\text { Race/Ethnicity: } \\
88.3 \% \text { non-Hispanic white; 3\% } \\
\text { non-Hispanic black; 5\% } \\
\text { Hispanic; } 2.3 \% \text { other } \\
\text { Background Diet: } \\
\text { NR }\end{array}$ & $\begin{array}{l}\text { Intervention/Exposure: } \\
\text { Age of CFB introduction: }<4,4-<6 \text {, and } \geq 6 \text { mo } \\
\text { CFB: Solid foods included dairy foods other than milk (e.g., yogurt, } \\
\text { cheese, and ice cream); soy foods other than soy milk (e.g. Tofu, } \\
\text { frozen soy desserts); baby cereal; other cereals and starches (e.g., } \\
\text { breakfa } \\
\text { Age: } 4-6 m o \\
\text { Assessment Methods: } \\
\text { Caregiver report } \\
\text { Outcomes: } \\
\text { Weight status } \\
\text { Age: } 6 y \\
\text { Assessment Methods: } \\
\text { Weight status: risk of obesity based on age- and sex-specific } \\
\text { BMl\%tile } 2000 \text { CDC; BMl calculated from height and weight } \\
\text { measured by mothers } \\
\text { Confounders accounted for: } \\
\text { Education:X } \\
\text { SES: } X \\
\text { Sex: } X \\
\text { Maternal age: } X \\
\text { Race/ethnicity: } X \\
\text { Feeding practices: } X \\
\text { Birth size: } X \\
\text { Gestational age: } X \\
\text { Other: Parity; Maternal BMl; Marital status; Infant intake of CFB }\end{array}$ & $\begin{array}{l}\text { Limitations: } \\
\text { Cannot determine whether groups } \\
\text { were similar at baseline on key } \\
\text { characteristics; Outcome assessors } \\
\text { were not blinded, both the independent } \\
\text { and dependent variables were } \\
\text { maternal self-report; Non-validated } \\
\text { measures used to assess outcome } \\
\text { Maternal self-report of IV/Exposure } \\
\text { and Outcomes; Sample not nationally } \\
\text { representative }\end{array}$ \\
\hline $\begin{array}{l}\text { Barton, } 2002 \\
\text { Prospective Cohort Study; U.S. } \\
\text { Sample Size: } \\
\text { Baseline } N=52\end{array}$ & $\begin{array}{l}\text { Intervention/Exposure: } \\
\text { Age of CFB introduction: }<4,>4 \mathrm{mo} \\
\text { CFB: "Solid foods" including infant cereal, vegetables, fruits, and } \\
<6 \text { oz fruit juice/d }\end{array}$ & $\begin{array}{l}\text { Limitations: } \\
\text { Cannot determine whether groups } \\
\text { differed at baseline on key } \\
\text { characteristics; Cannot determine }\end{array}$ \\
\hline
\end{tabular}




\begin{tabular}{|c|c|c|}
\hline $\begin{array}{l}\text { Analytic } \mathrm{N}=52 \\
\text { Attrition }=0 \% \\
\text { Power Analysis and Sufficient } \\
\text { Sample Size: NR } \\
\text { Sex: } \\
\text { RR } \\
\text { Race/Ethnicity: } \\
\text { NR } \\
\text { Background Diet: } \\
46 \% \text { BF, } 54 \% \text { FF at birth } \\
33 \% \text { BF, } 67 \% \text { FF at } 1-2 \mathrm{mo} \\
83 \% \text { received CFB }<4 \mathrm{mo}\end{array}$ & $\begin{array}{l}\text { Age: 4-6mo } \\
\text { Assessment Methods: } \\
\text { Caregiver report } \\
\text { Outcomes: } \\
\text { Weight; Length; HC } \\
\text { Age: } 1-2 m o ; 4-6 m o \\
\text { Assessment Methods: } \\
\text { Weight: measured by study personnel on balance-beam scale } \\
\text { Height: measured by trained personnel, on standard length board } \\
\text { HC: measured by study personnel with nonfabric measuring tape } \\
\text { Confounders accounted for: } \\
\text { None } \\
\text { Other: Weights, lengths, and HC at baseline NSD between groups }\end{array}$ & $\begin{array}{l}\text { whether outcome assessors were } \\
\text { blinded; Length of follow-up varied } \\
\text { from } 4 \text { to } 6 \text { mo of age; Cannot } \\
\text { determined validity/reliability of } \\
\text { measures used to assess outcomes } \\
\text { (single vs. multiple measures); Did not } \\
\text { adjust for any potential key } \\
\text { confounders (including education, } \\
\text { SES, sex, maternal age, race/ethnicity, } \\
\text { feeding practices, birth size, } \\
\text { gestational age) } \\
\text { Post-hoc tests suggest marginal } \\
\text { significance } \\
\text { Did not adjust for baseline values } \\
\text { though weight, length, and HC at } 1 \text { - } \\
2 \text { mo were similar between groups; } \\
\text { Groups were unbalanced (40 infants in } \\
\text { the CFB <4mo group vs. } 8 \text { infants in } \\
\text { the }>4 \text { mo group) } \\
\text { Cannot rule out reverse causality } \\
\text { (group already not gaining weight well) } \\
\text { Very short term followup }\end{array}$ \\
\hline $\begin{array}{l}\text { Butte, } 2000 \\
\text { Prospective Cohort Study; } \\
\text { United States } \\
\text { Sample Size: } \\
\text { Baseline } \mathrm{N}=76 \\
\text { Analytic } \mathrm{N}=72 \\
\text { Attrition }=5.3 \% \\
\text { Power Analysis and Sufficient } \\
\text { Sample Size: NR } \\
\text { Sex: } \\
56.6 \% \text { Female }\end{array}$ & $\begin{array}{l}\text { Intervention/Exposure: } \\
\text { Age of CFB introduction: Continuous } \\
\text { CFB: Solids other than formula, milk, juice, water, and vitamin- } \\
\text { mineral supplements } \\
\text { Age: } 3,6,12 \text {, and } 24 \mathrm{mo} \\
\text { Assessment Methods: } \\
\text { Food record, 3d, weighed } \\
\text { Outcomes: } \\
\text { Body composition; Weight; Length; Circumferences (head, chest, } \\
\text { arm, thigh) } \\
\text { Age: } 6-24 \text { mo }\end{array}$ & $\begin{array}{l}\text { Limitations: } \\
\text { Cannot determine whether groups } \\
\text { differed at baseline on key } \\
\text { characteristics; Did not adjust for } \\
\text { potential key confounders (education, } \\
\text { SES, maternal age, race/ethnicity, birth } \\
\text { size, gestational age) }\end{array}$ \\
\hline
\end{tabular}




\begin{tabular}{|c|c|c|}
\hline $\begin{array}{l}\text { Race/Ethnicity: } \\
72.4 \% \text { White, } 9.2 \% \text { Black, } \\
\text { 14.5\% Hispanic, 3.9\% Asian } \\
\text { Background Diet: } \\
\text { BF and FF infants were } \\
\text { analyzed separately } \\
\text { Mean age of introduction of } \\
\text { solids was } \sim 5 \text { mo for BF infants } \\
\text { and } \sim 4 \text { mo for FF infants }\end{array}$ & $\begin{array}{l}\text { Assessment Methods: } \\
\text { Body composition: } \\
\text { - Fat mass, fat-free mass calculated using a multicomponent body } \\
\text { composition model based on total body water by deuterium } \\
\text { dilution, total body potassium by whole body counting, and bone } \\
\text { mineral content by DXA } \\
\text { - Skinfold thickness (triceps, subscapular, flank, quadriceps): } \\
\text { measured by study personnel } \\
\text { Weight, length, circumferences (head, chest, arm, thigh) measured } \\
\text { by study personnel } \\
\text { Confounders accounted for: } \\
\text { Sex: } X \\
\text { Feeding practices: } X \\
\text { Other: Baseline anthropometric values }\end{array}$ & \\
\hline $\begin{array}{l}\text { Cantoral, } 2016 \\
\text { Prospective Cohort Study; } \\
\text { Mexico } \\
\text { Sample Size: } \\
\text { Baseline N: } 622 \text { (enrolled in } \\
\text { F/U) } \\
\text { Analytic N: } 227 \\
\text { Attrition: } 64 \% \\
\text { Power Analysis and Sufficient } \\
\text { Sample Size: NR } \\
\text { Sex: } \\
54 \% \text { female } \\
\text { Race/Ethnicity: } \\
\text { NR } \\
\text { Background Diet: } \\
31 \% \text { at least partial BF; } 73 \% \\
\text { introduced to SSB < } 12 \text { mo }\end{array}$ & $\begin{array}{l}\text { Intervention/Exposure: } \\
\text { Age of CFB introduction: } \leq 12 \text { vs }>12 \mathrm{mo} \\
\text { CFB: SSB: sum of daily intake of soda, commercial fruit drinks, } \\
\text { flavored water with sugar; Not including natural fruit or vegetable } \\
\text { juice } \\
\text { Age: } 12 \mathrm{mo} \text {; every } 6 \mathrm{mo} \text { until } 5 \mathrm{y} \text {; at } 8-14 \mathrm{y} \\
\text { Assessment Methods: } \\
116 \text {-item FFQ } \\
\text { Outcomes: } \\
\text { Weight status; WC } \\
\text { Age: } 8-14 y \\
\text { Assessment Methods: } \\
\text { Weight status: risk of obesity classified as BMl>2 SD of } z \text {-score; BMI } \\
\text { from measured weight (digital scale to } 0.1 \mathrm{~kg} \text { ) and height } \\
\text { (stadiometer to } 0.1 \mathrm{~cm} \text { ) } \\
\text { WC: measuring tape to } 0.1 \mathrm{~cm} \text { for abdominal obesity: WC } \geq 90 \text { th } \\
\% \text { Ctile } \\
\text { Confounders accounted for: } \\
\text { SES: } X\end{array}$ & $\begin{array}{l}\text { Limitations: } \\
\text { Cannot determine whether groups } \\
\text { differed at baseline on key } \\
\text { characteristics; Cannot determine if } \\
\text { outcome assessors were blinded; Did } \\
\text { not account for high attrition rate; Did } \\
\text { not adjust for key confounders of } \\
\text { education, maternal age, } \\
\text { race/ethnicity, birth size or gestational } \\
\text { age } \\
\text { Risk for measurement error associated } \\
\text { with FFQ; Assumes that SSB intake } \\
\text { was constant during the months } \\
\text { evaluated on FFQ }\end{array}$ \\
\hline
\end{tabular}




\begin{tabular}{|c|c|c|}
\hline & $\begin{array}{l}\text { Sex: } X \\
\text { Feeding practices: } X \\
\text { Other: non-SSB intake, TV watching, physical activity, maternal } \\
\text { obesity at } 12 \text { mo post-partum }\end{array}$ & \\
\hline $\begin{array}{l}\text { Carruth, } 2000 \\
\text { Prospective Cohort Study; U.S. } \\
\text { Sample Size: } \\
\text { Baseline } N=98 \\
\text { Analytic } N=94 \\
\text { Attrition = } 4.1 \% \\
\text { Power Analysis and Sufficient } \\
\text { Sample Size: Use of an } \\
\text { incomplete block design } \\
\text { maintained statistical power } \\
\text { Sex: } \\
48.0 \% \text { female (Skinner, } 1997) \\
\text { Race/Ethnicity: } \\
100 \% \text { White } \\
\text { Background Diet: } \\
83 \% \text { any BF, 33\% EBF at } 4 \text { mo, } \\
33 \% \text { MF at } 4 \text { mo; } 12 \% \text { EBF at } \\
6 \text { mo } \\
\sim 64 \% \text { CFB by } 4 \text { mo; Median } \\
\text { age for introducing CFB: cereal } \\
4.0 \text { (range .50-6.5 mo), juice } \\
4.50 \text { (1-11 mo), fruit } 5.0 \text { (.50- } \\
8.2 \text { mo), vegetables } 5.5 \text { (1-7.7 } \\
\text { mo) and the food cluster } \\
\text { (mixed foods, table foods, } \\
\text { meat) } 7.0 \text { (3-12 mo) }\end{array}$ & $\begin{array}{l}\text { Intervention/Exposure: } \\
\text { Age of CFB introduction: Continuous } \\
\text { CFB: supplementary foods included vegetables, cereal, juice, fruit, } \\
\text { and food cluster (meat, mixed food, or table food) } \\
\text { Age: } 2,3,4,6,8,10,12,16,20 \text {, and } 24 \mathrm{mo} \\
\text { Assessment Methods: } \\
24 \mathrm{~h} \text { recall } \\
\text { Outcomes: } \\
\text { Weight; Length } \\
\text { Age: } 2-8 m 0 ; 12-24 \mathrm{mo} \\
\text { Assessment Methods: } \\
\text { Weight: slope (kg/mo); standard protocol } \\
\text { Length: slope (cm/mo); standard protocol } \\
\text { Interview schedules were done using an incomplete block design, } \\
\text { such that the number of times measures were taken for each infant } \\
3-5 \text { times from } 2 \text { to } 10 \text { mo, and } 2-4 \text { times from } 12 \text { to } 24 \text { mo } \\
\text { Confounders accounted for: } \\
\text { Sex: } X \\
\text { Other: Final model } 2-8 m o \text { weight slope: gender; Final model } 2-8 m o \\
\text { length slope: gender; Final model } 2-8 m o \text { rate of change weight: } \\
\text { gender, length slope; Final model 12-24mo weight gain: length, pre- } \\
\text { existing weight }\end{array}$ & $\begin{array}{l}\text { Limitations: } \\
\text { Cannot determine whether groups } \\
\text { differed at baseline on key } \\
\text { characteristics; Cannot determine } \\
\text { whether outcome assessors were } \\
\text { blinded; Cannot determine adequacy } \\
\text { of statistical methods (previous } \\
\text { versions of regression models not } \\
\text { described, data not shown); Did not } \\
\text { adjust for potential key confounders } \\
\text { (education, SES, maternal age, } \\
\text { race/ethnicity, birth size, or gestational } \\
\text { age) } \\
\text { Limited generalizability (small sample } \\
\text { of white middle- to upper class } \\
\text { subjects from TN); Results section } \\
\text { reports that only age of introduction to } \\
\text { vegetables and food cluster remained } \\
\text { as significant predictors in rate of } \\
\text { change in relative weight but that ages } \\
\text { of CFB were not significant predictors } \\
\text { in weight gain } 12-24 \text { mo; Unclear if } \\
\text { cereal } \leq 4 \text { or }>4 \text { mo was analyzed in } \\
\text { relation to weight outcomes (reports in } \\
\text { relation to illness). Final models } \\
\text { reported significant predictors of weight } \\
\text { change but post-hoc removal of length } \\
\text { resulted in models becoming no longer } \\
\text { significant } \\
\text { Cannot rule out reverse causality }\end{array}$ \\
\hline
\end{tabular}




\begin{tabular}{|c|c|c|}
\hline $\begin{array}{l}\text { de Beer, } 2015 \\
\text { Prospective Cohort Study; The } \\
\text { Netherlands } \\
\text { Sample Size: } \\
\text { Baseline N: } 7863 \text { (gave birth; } \\
\text { N: 5551 with data; N:3321 } \\
\text { attended 5-6y visit) } \\
\text { Analytic N: } 2227 \\
\text { Attrition: 72\% } \\
\text { Power Analysis and Sufficient } \\
\text { Sample Size: NR } \\
\text { Sex: } \\
\sim 49 \% \text { Female } \\
\text { Race/Ethnicity: } \\
\sim 78 \% \text { Dutch } \\
\sim 2.4 \% \text { Surinamese } \\
\sim 2 \% \text { Turkish } \\
\sim 4 \% \text { Moroccan } \\
\sim 13 \% \text { Other } \\
\text { Background Diet: } \\
23 \%<1 \text { mo BF; 27\% BF 1- } \\
3 \text { mo; 31\% BF 3-mo; 19\% BF } \\
>6 \text { mo }\end{array}$ & $\begin{array}{l}\text { Intervention/Exposure: } \\
\text { Age of CFB introduction: }<4,4-6,>6 m o \\
\text { Age: } 1 \text {-11mo } \\
\text { Assessment Methods: } \\
\text { Monitored at check-ups } \\
\text { Outcomes: } \\
\text { Weight; Height; Body composition } \\
\text { z-score, height Z-score, fat-mass z-score; BMIZ } \\
\text { Age: } 6-12 m o ; \text {-6y } \\
\text { Assessment Methods: } \\
\text { Body composition: } \\
\text { - BMIZ calculated based on measured weight and height; z-score } \\
\text { - Fat mass (FMZ), fat free mass (FFMZ) Z-scores: calculated based } \\
\text { on total body water from measured arm-leg bioelectrical } \\
\text { impedance } \\
\text { Weight, height: measured via scale, length board, and portable } \\
\text { stadiometer; 'Conditional' sizes were created by standardized } \\
\text { residuals derived from regressions of current and prior height and } \\
\text { weight measures. Conditional height and weight represent a child's } \\
\text { deviation from expected size and growth } \\
\text { Confounders accounted for: } \\
\text { Education: X } \\
\text { Sex: X } \\
\text { Maternal age: X } \\
\text { Race/ethnicity: X } \\
\text { Feeding practices: } X \\
\text { Birth size: X (Birth weight) } \\
\text { Gestational age: X } \\
\text { Other: Current age; Childhood conditional height (12 months-5-6 } \\
\text { years); Conditional height and weight during all periods in infancy; } \\
\text { Maternal pre-pregnancy BMl; Maternal Height; Smoking; Alcohol; } \\
\text { Hypertension; Pregnancy duration as gestational age }\end{array}$ & $\begin{array}{l}\text { Limitations: } \\
\text { Cannot determine whether groups } \\
\text { differed at baseline on key } \\
\text { characteristics; Cannot determine if } \\
\text { outcome assessors or investigators } \\
\text { were blinded; Did not adjust for } \\
\text { potential key confounder (SES); High- } \\
\text { attrition rate not accounted for } \\
\text { High loss to follow-up and group } \\
\text { analyzed differed from original cohort } \\
\text { in ways NR; Anthropometric } \\
\text { measurements less precise than } \\
\text { DEXA; Reverse causality of infant } \\
\text { feeding and growth } \\
\text { Results reported in abstract and } \\
\text { discussion differ from results reported } \\
\text { in tables; No cut-off/P-values were } \\
\text { reported in tables related to analyses }\end{array}$ \\
\hline
\end{tabular}




\begin{tabular}{|c|c|c|}
\hline $\begin{array}{l}\text { Durmus, } 2012 \\
\text { Prospective Cohort Study; The } \\
\text { Netherlands } \\
\text { Sample Size: } \\
\text { Baseline N: } 1106 \\
\text { Analytic N: } 779 \\
\text { Attrition: } 29.6 \% \\
\text { Power Analysis and Sufficient } \\
\text { Sample Size: NR } \\
\text { Sex: } \\
\text { R9\% female } \\
\text { Race/Ethnicity: } \\
\text { Bac\% "Dutch ethnicity" } \\
\text { Background Diet: } \\
\text { duration }\end{array}$ & $\begin{array}{l}\text { Intervention/Exposure: } \\
\text { Age of CFB introduction: }<4,4-5,>5 \text { mo } \\
\text { CFB: Age that a fruit or vegetable snack was given } \\
\text { Age: } 2,6 \text {, and } 12 \text { mo } \\
\text { Assessment Methods: } \\
\text { Caregiver questionnaire } \\
\text { Outcomes: } \\
\text { Body composition (Adiposity) } \\
\text { Age: } 6 \text { mo, } 24 \text { mo } \\
\text { Assessment Methods: } \\
\text { Body composition: } \\
\text { - Subcutaneous fat mass via skinfold thickness (biceps, triceps, } \\
\text { suprailiacal, subscapular) measured by study personnel using } \\
\text { calipers } \\
\text { Confounders accounted for: } \\
\text { Education: } X \\
\text { Sex: } X \\
\text { Feeding practices: } X \\
\text { Birth size: } X \text { (Birth weight) } \\
\text { Gestational age: } X \\
\text { Other: Maternal BMI, Smoking; Parity; Current height; Observer }\end{array}$ & $\begin{array}{l}\text { Limitations: } \\
\text { Cannot determine whether groups } \\
\text { were similar at baseline on all key } \\
\text { characteristics; Cannot determine } \\
\text { whether outcome assessors were } \\
\text { blinded; Did not assess the impact of } \\
\text { high loss to follow-up ( 27\%); Did not } \\
\text { adjust for key confounders (SES, } \\
\text { maternal age, race/ethnicity) } \\
\text { BF information only available in } 78 \% \text { of } \\
\text { sample; CFB included only a fruit or } \\
\text { vegetable snack and other products } \\
\text { could have been introduced; } \\
\text { Homogenous, highly-educated sample; } \\
\text { Limited generalizability }\end{array}$ \\
\hline $\begin{array}{l}\text { Durmus, } 2014 \\
\text { Prospective Cohort Study; The } \\
\text { Netherlands } \\
\text { Sample Size: } \\
\text { Baseline N: } 6054 \\
\text { Analytic N: } 5063 \\
\text { Attrition: } 16.4 \% \\
\text { Power Analysis and Sufficient } \\
\text { Sample Size: NR } \\
\text { Sex: } \\
50.2 \% \text { Female }\end{array}$ & $\begin{array}{l}\text { Intervention/Exposure: } \\
\text { Age of CFB introduction: }<4,4-4.9, \geq 5 \mathrm{mo} \\
\text { CFB: Age that a fruit or vegetable snack was given } \\
\text { Age: } 2,6 \text {, and } 12 \mathrm{mo} \\
\text { Assessment Methods: } \\
\text { Caregiver questionnaire } \\
\text { Outcomes: } \\
\text { Weight status; Body composition; WC (abdominal fat) } \\
\text { Age: } 6 y \\
\text { Assessment Methods: } \\
\text { Weight status: risk of overweight or obesity based on IOTF } \\
\text { categories for BMl }\end{array}$ & $\begin{array}{l}\text { Limitations: } \\
\text { Cannot determine whether groups } \\
\text { were similar at baseline on all key } \\
\text { characteristics; Cannot determine } \\
\text { whether outcome assessors were } \\
\text { blinded; Did not adjust for key } \\
\text { confounders (SES or feeding } \\
\text { practices) } \\
\text { Did not collect information on BM } \\
\text { consumption or composition }\end{array}$ \\
\hline
\end{tabular}




\begin{tabular}{|c|c|c|}
\hline $\begin{array}{l}\text { Race/Ethnicity: } \\
\text { 100\% "Dutch ethnicity" } \\
\text { Background Diet: } \\
92.4 \% \text { ever BF; } 20.4 \% \text { EBF to } \\
4 \mathrm{mo}\end{array}$ & $\begin{array}{l}\text { Body composition: } \\
\text { - Adiposity: total fat mass, android-gynoid ratio (DXA) } \\
\text { - BMI: calculated from measured height/weight (stadiometer/ } \\
\text { mechanical scale) } \\
\text { Abdominal/WC: abdomial fat via ultra-sound } \\
\text { Confounders accounted for: } \\
\text { Education: } X \\
\text { Sex: } X \\
\text { Maternal age: } X \\
\text { Race/ethnicity: X } \\
\text { Birth size: } X \\
\text { Gestational age: } X \\
\text { Other: Child age, Child height; Parity; Maternal BMI, Smoking; TV } \\
\text { watching }\end{array}$ & \\
\hline $\begin{array}{l}\text { Ejlerskov, } 2015 \\
\text { Prospective Cohort Study; } \\
\text { Denmark } \\
\text { Sample Size: } \\
\text { Baseline } N=330 \\
\text { Analytic } N=233 \\
\text { Attrition = } 29.4 \% \\
\text { Power Analysis and Sufficient } \\
\text { Sample Size: } \mathrm{N}=260 \text { to detect } \\
\text { differences in growth } \\
\text { Sex: } \\
52.4 \% \text { Female } \\
\text { Race/Ethnicity: } \\
\text { NR } \\
\text { Background Diet: } \\
\text { EBF: } 126 d, \text { median (IQR=91- } \\
\text { 152); fully BF at } 4 \text { mo: } 67.8 \% ; \\
\text { PBF at 9mo: } 42.5 \% \text { median } \\
\text { g/day formula at } 9 \text { mo: } 169 \\
\text { (IQR=0-410); median mo }\end{array}$ & $\begin{array}{l}\text { Intervention/Exposure: } \\
\text { Age of CFB introduction: earliest age receiving one of } 19 \text { food } \\
\text { categories in mo; } \\
\text { Age: } 9,18 \text {, and } 36 \text { mo } \\
\text { Assessment Methods: } \\
\text { Parental interview } \\
\text { Outcomes: } \\
\text { Body composition } \\
\text { Age: } 36 \text { mo }( \pm 3) \\
\text { Assessment Methods: } \\
\text { Body composition: } \\
\text { - Fat free mass index, fat mass index: calculated from BIA and DXA } \\
\quad \text { measures for fat mass and fat free mass } \\
\text { Confounders accounted for: } \\
\text { Education: } X \\
\text { Sex: } X \\
\text { Feeding practices: } X \text { (Table } 7) \\
\text { Birth size: } X \\
\text { Other: WAZ 0-5mo; Maternal BMI }\end{array}$ & $\begin{array}{l}\text { Limitations: } \\
\text { Cannot determine whether groups } \\
\text { were similar at baseline on key } \\
\text { characteristics; Cannot determine } \\
\text { whether outcome assessors were } \\
\text { blinded; Cannot determine validity and } \\
\text { reliability of dietary assessment; Did } \\
\text { not assess the impact of high loss to } \\
\text { follow-up ( 29\%); Did not adjust for } \\
\text { key confounders such as SES, } \\
\text { maternal age, race/ethnicity, or } \\
\text { gestational age } \\
\text { Insufficient power; prediction error of } \\
\text { FFM and FM from BIA vs. DXA; age of } \\
\text { introduction to CFB collected } \\
\text { retrospectively; Fully BF for } 6 \text { mo group } \\
\text { drove results; }\end{array}$ \\
\hline
\end{tabular}




\begin{tabular}{|c|c|c|}
\hline $\begin{array}{l}\text { introduction of solids: } 4.0 \\
(I Q R=4.0-5.0)\end{array}$ & & \\
\hline $\begin{array}{l}\text { Fairley, } 2015 \\
\text { Prospective Cohort Study; } \\
\text { United Kingdom } \\
\text { Sample Size: } \\
\text { Baseline N: } 1707 \\
\text { Analytic N: } 987 \\
\text { Attrition: } 42 \% \\
\text { Power Analysis and Sufficient } \\
\text { Sample Size: NR } \\
\text { Sex: } \\
\text { NR } \\
\text { Race/Ethnicity: } \\
39 \% \text { White British } \\
48 \% \text { Pakistani } \\
\text { 13\% Other } \\
\text { Background Diet: } \\
27 \% \text { never BF; } 26.3 \% \text { BF } \\
<1 \text { mo; 18.5\% BF } 1-4 \mathrm{mo} ; 28.2 \% \\
\text { BF >4mo }\end{array}$ & $\begin{array}{l}\text { Intervention/Exposure: } \\
\text { Age of CFB introduction: }<17 \text { or } \geq 17 \mathrm{wk} \\
\text { Age: } 6 \text { and } 12 \mathrm{mo} \\
\text { Assessment Methods: } \\
\text { Caregiver questionnaire } \\
\text { Outcomes: } \\
\text { Weight status; body composition } \\
\text { Age: } 36 \text { mo } \\
\text { Assessment Methods: } \\
\text { Weight status: relative risk of overweight classified as BMIZ } \geq 85 \text { th } \\
\text { \%tile } \\
\text { Body composition: BMI Z-scores by WHO, } 2006 \text { standards from } \\
\text { weight and height } \\
\text { Confounders accounted for: } \\
\text { Education: } X \\
\text { Sex: } X \\
\text { Maternal age: } X \\
\text { Race/ethnicity: } X \\
\text { Feeding practices: } X \\
\text { Birth size: } X \\
\text { Gestational age: } X \\
\text { Other: Parity; Delivery Mode }\end{array}$ & $\begin{array}{l}\text { Limitations: } \\
\text { Cannot determine whether groups } \\
\text { were similar at baseline on key } \\
\text { characteristics; Cannot determine } \\
\text { whether outcome assessors were } \\
\text { blinded; Cannot determine } \\
\text { validity/reliability of outcome } \\
\text { assessment; Did not assess the impact } \\
\text { of high loss to follow-up ( } \sim 2 \%) \text {; Did } \\
\text { not adjust analyses for SES } \\
\text { Insufficient power to examine Obesity; } \\
\text { Multiple testing not accounted for }\end{array}$ \\
\hline $\begin{array}{l}\text { Fawzi, } 1997 \\
\text { Prospective Cohort Study; } \\
\text { Israel } \\
\text { Sample Size: } \\
\text { Baseline N: } 1041 \\
\text { Analytic N: } 351 \\
\text { Attrition: } 66 \% \\
\text { Power Analysis and Sufficient } \\
\text { Sample Size: NR }\end{array}$ & $\begin{array}{l}\text { Intervention/Exposure: } \\
\text { Age of CFB introduction at } 1,2 \text {, or } 3 \text { mo vs. EBF } \\
\text { CFB: solids exclusively } \\
\text { Age: Birth, } 1-3 \text { mo } \\
\text { Assessment Methods: } \\
\text { Maternal interview } \\
\text { Outcomes: } \\
\text { Weight } \\
\text { Age: } 2 \mathrm{mo}, 3 \mathrm{mo}\end{array}$ & $\begin{array}{l}\text { Limitations: } \\
\text { Cannot determine whether groups } \\
\text { were similar at baseline on key } \\
\text { characteristics; Cannot determine } \\
\text { whether outcome assessors were } \\
\text { blinded; Cannot determine } \\
\text { validity/reliability of outcome } \\
\text { assessment; Did not adjust for } \\
\text { potential confounders of SES, maternal } \\
\text { age, race/ethnicity, or gestational age; }\end{array}$ \\
\hline
\end{tabular}




\begin{tabular}{|c|c|c|}
\hline $\begin{array}{l}\text { Sex: } \\
\text { NR } \\
\text { Race/Ethnicity: } \\
49 \% \text { Israel } 51 \% \text { immigrants } \\
\text { from North African country } \\
\text { Background Diet: } \\
92.3 \% \text { EBF at birth; } 35.9 \% \text { EBF } \\
\text { at } 1 \mathrm{mo} ; 19.6 \% \text { EBF at } 2 \mathrm{mo} ; \\
7.3 \% \text { EBF at } 3 \mathrm{mo} ; 0.7 \% \text { EBF at } \\
6 \mathrm{mo}\end{array}$ & $\begin{array}{l}\text { Assessment Methods: } \\
\text { Weight: gain; beam-balance scales to } 0.1 \mathrm{~kg} \\
\text { Confounders accounted for: } \\
\text { Education: } X \\
\text { Sex: } X \\
\text { Feeding practices: } X \text { (in analyses) } \\
\text { Birth size: } X \text { (Birth weight, weight at first prenatal visit) } \\
\text { Other: Maternal height }\end{array}$ & $\begin{array}{l}\text { Did not account for only } 2 \text { infants being } \\
\text { EBF at } 6 \text { mo so could not do } \\
\text { comparisons as planned } \\
\text { Cannot rule out reverse causality } \\
\text { Limited generalizability due to } \\
\text { immigrant sample } \\
\text { Short-term follow-up }\end{array}$ \\
\hline $\begin{array}{l}\text { Ferris, } 1980 \\
\text { Prospective Cohort Study; } \\
\text { United States } \\
\text { Sample Size: } \\
\text { Baseline N: } 92 \\
\text { Analytic N: } 92 \\
\text { Attrition: } 0 \% \\
\text { Power Analysis and Sufficient } \\
\text { Sample Size: NR } \\
\text { Sex: } \\
\text { 100\% Female } \\
\text { Race/Ethnicity: } \\
\text { NR } \\
\text { Background Diet: } \\
43.5 \% \text { BF alone; } 13 \% \\
\text { BF+solids; } 11 \% \text { FF alone; } \\
21.7 \% \text { FF+solids }\end{array}$ & $\begin{array}{l}\text { Intervention/Exposure: } \\
\text { Age of CFB introduction: at } 2 \mathrm{mo} \text { or }>2 \mathrm{mo} \\
\text { CFB: CFB+FF vs. FF alone } \\
\text { Age: } 2 \mathrm{mo} \\
\text { Assessment Methods: } \\
\text { Food record, } 3 \mathrm{~d} \text { and diary } \\
\text { Outcomes: } \\
\text { Body composition; Weight; Length } \\
\text { Age: birth-6mo } \\
\text { Assessment Methods: } \\
\text { Body composition: weight/length }{ }^{2} \text { from measures } \\
\text { Weight, and height with scale and infantometer } \\
\text { Confounders accounted for: } \\
\text { SES: } X \\
\text { Sex: } X \text { (all female) } \\
\text { Birth size: } X \\
\text { Other: Income, maternal smoking, parent weight, and parity at } \\
\text { baseline similar between groups }\end{array}$ & $\begin{array}{l}\text { Limitations: } \\
\text { Cannot determine whether outcome } \\
\text { assessors were blinded; Did not adjust } \\
\text { analyses for potential key confounders } \\
\text { (education, race/ethnicity, or } \\
\text { gestational age) } \\
\text { Sample was } 100 \% \text { female - male } \\
\text { participants were to be reported } \\
\text { elsewhere; Feeding practices part of } \\
\text { analyses }\end{array}$ \\
\hline $\begin{array}{l}\text { Flores, 2013a } \\
\text { Prospective Cohort Study; U.S. } \\
\text { Sample Size: } \\
\text { Baseline N: } 14000 \\
\text { Analytic N: } 6800 \\
\text { Attrition: } \sim 51 \%\end{array}$ & $\begin{array}{l}\text { Intervention/Exposure: } \\
\text { Age of CFB introduction: Continuous, mo; }<3.5 \text { vs. } \geq 3.5 \mathrm{mo} \\
\text { CFB: solid food a/o finger food } \\
\text { Age: NR } \\
\text { Assessment Methods: } \\
\text { Caregiver a/o teacher report }\end{array}$ & $\begin{array}{l}\text { Limitations: } \\
\text { Cannot determine whether groups } \\
\text { were similar at baseline on key } \\
\text { characteristics; Cannot determine } \\
\text { whether outcome assessors were } \\
\text { blinded; Did not assess the impact of }\end{array}$ \\
\hline
\end{tabular}




\begin{tabular}{|c|c|c|}
\hline $\begin{array}{l}\text { Power Analysis and Sufficient } \\
\text { Sample Size: NR } \\
\text { Sex: } \\
48.9 \% \text { Female } \\
\text { Race/Ethnicity: } \\
54 \% \text { White; } 25 \% \text { Latino; } 14 \% \\
\text { African- American; } 2.7 \% \\
\text { Asian/PI; 0.4\% Al/AN; 3.8\% } \\
\text { Multi } \\
\text { Background Diet: } \\
\text { Average duration BF } ~ 3.7 m o \\
\text { Average introduction to formula } \\
\sim 3.5 \text { mo }\end{array}$ & $\begin{array}{l}\text { Outcomes: } \\
\text { Weight status } \\
\text { Age: Kindergarten entry ( 7.5y) } \\
\text { Assessment Methods: } \\
\text { Weight status: prevalance of overweight, based on age- and sex- } \\
\text { specific BMl } \geq 85 \text { th \%tile; BMI by CDC standards, calculated from } \\
\text { measured weight, and height (in triplicate with a digital scale and } \\
\text { stadiometer) } \\
\text { Confounders accounted for: } \\
\text { None } \\
\text { Recursive partioning analyses accounted for: BMl at preschool age } \\
\text { and at 2y, race/ethnicity, maternal gestational diabetes, birth weight }\end{array}$ & $\begin{array}{l}\text { high loss to follow-up ( } 51 \%) \text {; Did not } \\
\text { adjust analyses for potential key } \\
\text { confounders (education, SES, sex, } \\
\text { maternal age, or gestational age) } \\
\text { RPA selects highest significance for } \\
\text { binary split based on reducing variance } \\
\text { not by P values; groups varied by } \\
\text { outcome status in all risk factors } \\
\text { assessed in bivariate analyses. }\end{array}$ \\
\hline $\begin{array}{l}\text { Flores, 2013b } \\
\text { Prospective Cohort Study; } \\
\text { United States } \\
\text { Sample Size: } \\
\text { Baseline N: } 14000 \\
\text { Analytic N: } 6800 \\
\text { Attrition: } 51 \% \\
\text { Power Analysis and Sufficient } \\
\text { Sample Size: NR } \\
\text { Sex: } \\
48.9 \% \text { Female } \\
\text { Race/Ethnicity: } \\
54 \% \text { White } \\
25 \% \text { Latino } \\
\text { 14\% African- American } \\
2.7 \% \text { Asian/PI } \\
0.4 \% \text { Al/AN } \\
3.8 \% \text { Multi } \\
\text { Background Diet: } \\
\text { Average duration BF 3.7mo }\end{array}$ & $\begin{array}{l}\text { Intervention/Exposure: } \\
\text { Age of CFB introduction: Continuous, mo } \\
\text { CFB: solid food a/o finger food } \\
\text { Age: NR } \\
\text { Assessment Methods: } \\
\text { Caregiver a/o teacher report } \\
\text { Outcomes: } \\
\text { Weight status } \\
\text { Age: Kindergarten entry ( 7.5y) } \\
\text { Assessment Methods: } \\
\text { Weight status: prevalance of obesity classified as BMI } \geq 99 \text { th \%tile; } \\
\text { age- and sex- specific BMI by CDC standards calculated from } \\
\text { weight, and height, which were measured in triplicate with a digital } \\
\text { scale and stadiometer } \\
\text { Confounders accounted for: } \\
\text { Feeding practices: } X \text { (in model) }\end{array}$ & $\begin{array}{l}\text { Limitations: } \\
\text { Cannot determine whether groups } \\
\text { were similar at baseline on key } \\
\text { characteristics; Cannot determine } \\
\text { whether outcome assessors were } \\
\text { blinded; Did not assess the impact of } \\
\text { high loss to follow-up ( } 51 \%) \text {; Did not } \\
\text { adjust analyses for potential } \\
\text { confounders (education, SES, sex, } \\
\text { maternal age, race/ethnicity, birth size } \\
\text { or gestational age) } \\
\text { Lack of information provided on } \\
\text { intervention/exposure measurement; } \\
\text { Feeding practices included in model }\end{array}$ \\
\hline
\end{tabular}




\begin{tabular}{|c|c|c|}
\hline $\begin{array}{l}\text { Average introduction to formula } \\
\sim 3.5 \mathrm{mo}\end{array}$ & & \\
\hline $\begin{array}{l}\text { Forsyth, } 1993 \\
\text { Prospective Cohort Study ; } \\
\text { Scotland } \\
\text { Sample Size: } \\
\text { Baseline N: } 671 \\
\text { Analytic N: } 584 \text { at 8wk; } 576 \text { at } \\
\text { 13wk; } 544 \text { at } 26 w k ; 548 \text { at } \\
52 w k ; 392 \text { at 104wk } \\
\text { Attrition: } 13 \% \text { at } 8 w k ; ~ 14 \% \\
\text { at 13wk; 19\% at 26wk; 18\% } \\
\text { at 52wk; 42\% at 104wk } \\
\text { Sample Size Calculation: NR } \\
\text { Sex: } \\
\text { 52\% Female } \\
\text { Race/Ethnicity: } \\
\text { NR } \\
\text { Background Diet: } \\
14 \% \text { EBF, 19.3\% PBF, 66\% } \\
\text { MF }\end{array}$ & $\begin{array}{l}\text { Intervention/Exposure: } \\
\text { Age of CFB introduction: }<8,8-12 \text {, or }>12 w k \\
\text { Age: } 8-12 w k(\sim 2-3 m o) \\
\text { Assessment Methods: } \\
\text { Maternal questionnaire } \\
\text { Outcomes: } \\
\text { Weight } \\
\text { Age: } 4,8,13,26,52,104 w k \\
\text { Assessment Methods: } \\
\text { Weight: weighing machine with } 20 \mathrm{~g} \text { divisions } \\
\text { Confounders accounted for: } \\
\text { SES: } X \\
\text { Sex: } X \\
\text { Maternal age: } X \\
\text { Feeding practices: } X \\
\text { Birth size: X (Birth weight) } \\
\text { Gestational age: All full-term } \\
\text { Other: Smoking; Atopy risk }\end{array}$ & $\begin{array}{l}\text { Limitations: } \\
\text { Groups differed at baseline on key } \\
\text { characteristics but analyses were not } \\
\text { adjusted for differences; Cannot } \\
\text { determine reliability/validity of outcome } \\
\text { assessment; Did not adjust analyses } \\
\text { for potential key confounders } \\
\text { (education, race/ethnicity, or } \\
\text { gestational age). } \\
\text { Cannot rule out reverse causality } \\
\text { (males earlier CFB and birth weights } \\
>4 \mathrm{~kg} \text { ) }\end{array}$ \\
\hline $\begin{array}{l}\text { Friel, } 1985 \\
\text { Prospective Cohort Study; } \\
\text { Canada } \\
\text { Sample Size: } \\
\text { Baseline } \mathrm{N}=110 \text { (50 pre-term, } \\
60 \text { full-term) } \\
\text { Analytic } \mathrm{N}=110 \\
\text { Attrition }=\mathrm{N} / \mathrm{A} \\
\text { Power Analysis and Sufficient } \\
\text { Sample Size: NR } \\
\text { Sex: } \\
48 \% \text { Female } \\
\text { Race/Ethnicity: }\end{array}$ & $\begin{array}{l}\text { Intervention/Exposure: } \\
\text { Age of first CFB introduction (NR) } \\
\text { Age: } 6 \mathrm{mo} \\
\text { Assessment Methods: } \\
\text { Diet record, 3d } \\
\text { Outcomes: } \\
\text { Weight } \\
\text { Age: } 12 \mathrm{mo} \\
\text { Assessment Methods: } \\
\text { Weight: spring balance (pre-term); beam balance (full-term) to } 50 \mathrm{~g} \\
{ }^{*} \text { Only weight at } 12 \text { mo reported in relation to CFB } \\
\text { Confounders accounted for: } \\
\text { SES: } \mathrm{X}\end{array}$ & $\begin{array}{l}\text { Limitations: } \\
\text { Cannot determine if inclusion/exclusion } \\
\text { criteria were similar across groups; } \\
\text { Cannot determine whether groups } \\
\text { were similar at baseline on key } \\
\text { characteristics; Cannot determine } \\
\text { whether outcome assessors were } \\
\text { blinded; Did not adjust analyses for } \\
\text { potential key confounders (maternal } \\
\text { age though groups were similar, } \\
\text { education, race/ethnicity, or feeding } \\
\text { practices) } \\
\text { Only reported outcomes in relation to } \\
\text { weight at } 12 \text { mo and cross-sectional }\end{array}$ \\
\hline
\end{tabular}




\begin{tabular}{|c|c|c|}
\hline $\begin{array}{l}\text { Background Diet: } \\
12 \% \text { (pre-term) and } 47 \% \text { (full- } \\
\text { term) BF at 3mo } \\
\text { At } 6 \text { mo, } 10 \% \text { (pre-term) and } \\
25 \% \text { (full-term) given cow's milk } \\
\text { Pre-term mixed-fed earlier than } \\
\text { full-term infants (at 166d vs. } \\
85 d \text { ) }\end{array}$ & $\begin{array}{l}\text { Sex: } X \\
\text { Birth size: } X \\
\text { Gestational age: } X \\
\text { Other: Maternal age was similar between groups }\end{array}$ & $\begin{array}{l}\text { analysis of age of introduction to CFB } \\
\text { and length at } 3 \mathrm{mo}\end{array}$ \\
\hline $\begin{array}{l}\text { Gaffney, } 2012 \\
\text { Prospective Cohort Study; U.S. } \\
\text { Sample Size: } \\
\text { Baseline } N=4902 \\
\text { Analytic } N=691 \\
\text { Attrition = 85.9\% } \\
\text { Power Analysis and Sufficient } \\
\text { Sample Size: NR } \\
\text { Sex: } \\
49.3 \% \text { Female } \\
\text { Race/Ethnicity: } \\
\text { NR } \\
\text { Background Diet: } \\
\text { BF intensity in late infancy: } \\
52.1 \% \text { low, 24.3\% medium, } \\
23.6 \% \text { high }\end{array}$ & $\begin{array}{l}\text { Intervention/Exposure: } \\
\text { Age of CFB introduction: }<4,4-6 \text {, and }>/=6 \mathrm{mo} \\
\text { Age: } 1,2,3,4,5,6,7,9,10 \text {, and } 12 \mathrm{mo} \\
\text { Assessment Methods: } \\
\text { Maternal questionnaire } \\
\text { Outcomes: } \\
\text { Weight } \\
\text { Age: } 12 \mathrm{mo} \\
\text { Assessment Methods: } \\
\text { Weight: WAZ via maternal-report of weight from recent doctor visit; } \\
\text { calculated age and sex-specific } z \text { scores by CDC standards } \\
\text { Confounders accounted for: } \\
\text { Education: } X \\
\text { SES: } X \\
\text { Maternal age: } X \\
\text { Race/ethnicity: } X \\
\text { Feeding practices: } X \\
\text { Birth size: } X \text { (Birth weight) } \\
\text { Other: Mother's smoking status, weight gain in pregnancy, pregravid } \\
\text { BMl }\end{array}$ & $\begin{array}{l}\text { Limitations: } \\
\text { Cannot determine whether groups } \\
\text { were similar at baseline on key } \\
\text { characteristics; Cannot determine } \\
\text { reliability/validity of outcome } \\
\text { assessment and outcome assessors } \\
\text { were not blinded due to both feeding } \\
\text { and outcome data self-reported by } \\
\text { mothers; Did not assess the impact of } \\
\text { high loss to follow-up ( } 40 \%) \text {; Did not } \\
\text { adjust analyses for potential } \\
\text { confounders (sex, gestational age) } \\
\text { Generalizability limited due to sample } \\
\text { not being representative (Data not } \\
\text { derived from a nationally } \\
\text { representative sample, } \\
\text { underrepresented minority groups; } \\
\text { sample biased toward mothers with } \\
\text { more education and higher SES) }\end{array}$ \\
\hline $\begin{array}{l}\text { Garden, } 2012 \\
\text { Prospective Cohort Study; } \\
\text { Australia } \\
\text { Sample Size: } \\
\text { Baseline N: } 616\end{array}$ & $\begin{array}{l}\text { Intervention/Exposure: } \\
\text { Age of CFB introduction: } \leq 3 \text { or }>3 \mathrm{mo} \\
\text { CFB: solid food ever } \\
\text { Age: } 3 \mathrm{mo} \\
\text { Assessment Methods: } \\
\text { Questionnaire }\end{array}$ & $\begin{array}{l}\text { Limitations: } \\
\text { Cannot determine whether groups } \\
\text { were similar on baseline } \\
\text { characteristics; Cannot determine } \\
\text { whether outcome assessors were } \\
\text { blinded; Did not account for high } \\
\text { attrition rate/lost to follow-up (40\%); }\end{array}$ \\
\hline
\end{tabular}




\begin{tabular}{|c|c|c|}
\hline $\begin{array}{l}\text { Analytic N: } 370 \text { (at 3mo, } \\
\text { Timing) Analytic N: } 298 \text { (at } \\
\text { 18mo, Type) } \\
\text { Attrition: 40\% (3mo) } \\
\text { Attrition: } 51.6 \% \text { (18mo) } \\
\text { Power Analysis and Sufficient } \\
\text { Sample Size: NR } \\
\text { Sex: } \\
49 \% \text { female } \\
\text { Race/Ethnicity: } \\
\text { NR } \\
\text { Background Diet: } \\
40 \% \text { BF 0-3mo; } 16.8 \% \text { BF 3- } \\
6 \mathrm{mo} ; 43.2 \% \text { BF } \geq 6 \mathrm{mo}\end{array}$ & 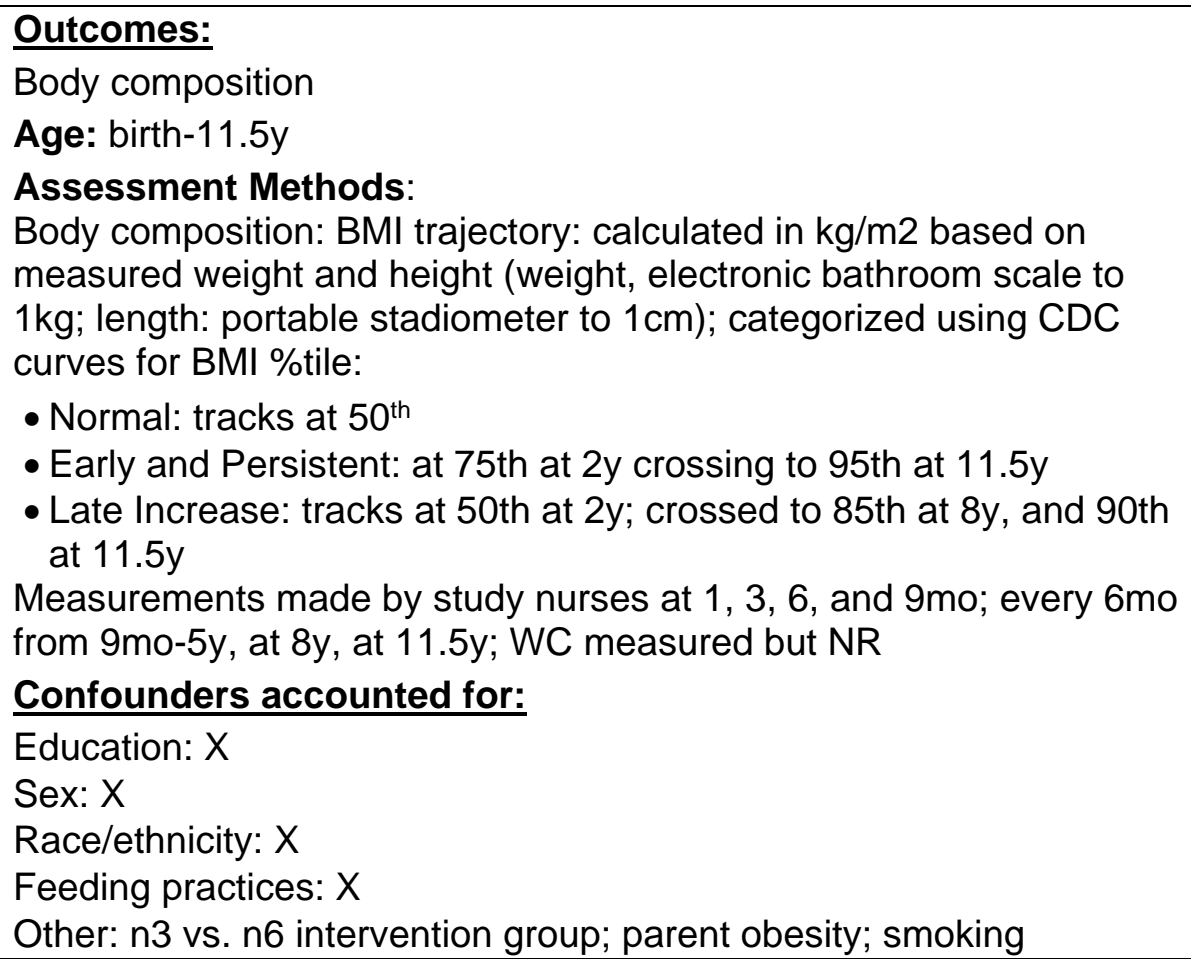 & $\begin{array}{l}\text { Did not adjust for potential key } \\
\text { confounders (SES, maternal age, birth } \\
\text { size, gestational age) }\end{array}$ \\
\hline $\begin{array}{l}\text { Gibbs, } 2014 \\
\text { Prospective Cohort Study; U.S. } \\
\text { Sample Size: } \\
\text { Baseline } N=10,500 \\
\text { Analytic } N=7,880 \\
\text { Attrition = } 25 \% \\
\text { Power Analysis and Sufficient } \\
\text { Sample Size: NR } \\
\text { Sex: } \\
50 \% \text { Female } \\
\text { Race/Ethnicity: } \\
50 \% \text { White; } 16 \% \text { Black, } 18 \% \\
\text { Hispanic, } 10 \% \text { Asian, and } 11 \% \\
\text { other }\end{array}$ & $\begin{array}{l}\text { Intervention/Exposure: } \\
\text { Age of CFB introduction: }<4 \mathrm{mo} \text { or not }<4 \mathrm{mo} \\
\text { Age: } 9 \mathrm{mo} \\
\text { Assessment Methods: } \\
\text { Maternal interview } \\
\text { Outcomes: } \\
\text { Weight status (Obesity) } \\
\text { Age: } 24 \mathrm{mo} \\
\text { Assessment Methods: } \\
\text { Weight status: risk of obesity, defined as } 98 \text { th \%tile for weight; also } \\
\text { reported by WHO and CDC standards and by International Obesity } \\
\text { Task Force (IOTF) cut-offs low/high BMI by sex according to } \\
\text { measured weight } \\
\text { Confounders accounted for: } \\
\text { Education: } X\end{array}$ & $\begin{array}{l}\text { Limitations: } \\
\text { Cannot determine whether groups } \\
\text { were similar on baseline } \\
\text { characteristics; Did not account for } \\
\text { high attrition rate/lost to follow-up } \\
(25 \%)\end{array}$ \\
\hline
\end{tabular}




\begin{tabular}{|c|c|c|}
\hline $\begin{array}{l}\text { Background Diet: } \\
28 \% \text { BF for } \geq 3 \mathrm{mo}, 16 \% \mathrm{BF} \\
\geq 6 \mathrm{mo}, 12 \% \text { BF } 3-5 \mathrm{mo}\end{array}$ & $\begin{array}{l}\text { SES: } X \\
\text { Sex: } X \\
\text { Maternal age: } X \\
\text { Race/ethnicity: } X \\
\text { Feeding practices: } X \\
\text { Birth size: } X \text { (Birth weight) } \\
\text { Gestational age: } X \\
\text { Other: Smoking; Depression, Model } 1 \text { or } 3 \text { : both biological parents in } \\
\text { home, sibling size, mother US born, mother's BMI, twin status, } \\
\text { daycare }\end{array}$ & \\
\hline $\begin{array}{l}\text { Griffiths, } 2009 \\
\text { Prospective Cohort Study; } \\
\text { United Kingdom } \\
\text { Sample Size: } \\
\text { Baseline N: } 18,296 \\
\text { Analytic N: 10,533 } \\
\text { Attrition: 42\% } \\
\text { Power Analysis and Sufficient } \\
\text { Sample Size: NR } \\
\text { Sex: } \\
50.1 \% \text { Female } \\
\text { Race/Ethnicity: } \\
\text { NR } \\
\text { Background Diet: } \\
2.1 \% \text { never BF; } 67.9 \% \text { ever } \\
\text { BF; } 60.1 \% \text { BF <4mo; } 39.9 \% \text { BF } \\
\geq 4 \text { mo }\end{array}$ & $\begin{array}{l}\text { Intervention/Exposure: } \\
\text { Age of CFB introduction: }<4 \text { vs. } \geq 4 \mathrm{mo} \\
\text { Age: } 9 \text { mo } \\
\text { Assessment Methods: } \\
\text { Parental interview } \\
\text { Outcomes: } \\
\text { Weight } \\
\text { Age: birth-3y } \\
\text { Assessment Methods: } \\
\text { Weight: conditional weight gain from measured weight (Tanita } \\
\text { scales) and z scores by British } 1990 \text { standards } \\
\text { Confounders accounted for: } \\
\text { Education: } X \\
\text { SES: } X \\
\text { Sex: } X \\
\text { Feeding practices: } X \\
\text { Other: Smoking; Parental BMI; Current child height 3y }\end{array}$ & $\begin{array}{l}\text { Limitations: } \\
\text { Cannot determine whether groups } \\
\text { were similar on baseline } \\
\text { characteristics; Cannot determine if } \\
\text { outcome assessors were blinded; } \\
\text { Cannot determine validity/reliability of } \\
\text { outcome assessment; Did not adjust } \\
\text { for potential key confounders (maternal } \\
\text { age, race/ethnicity, birth size, or } \\
\text { gestational age) } \\
\text { Retrospective recall; reverse causality } \\
\text { Results were significant before } \\
\text { adjustment for current child height z- } \\
\text { score (not a key confounder); } \\
\text { Race/ethnicity NR appears sample } \\
\text { was } 100 \% \text { white }\end{array}$ \\
\hline $\begin{array}{l}\text { Griffiths, } \mathbf{2 0 1 0} \\
\text { Prospective Cohort Study; } \\
\text { United Kingdom } \\
\text { Sample Size: } \\
\text { Baseline N: } 18,296 \\
\text { Analytic N: } 11,653\end{array}$ & $\begin{array}{l}\text { Intervention/Exposure: } \\
\text { Age of CFB introduction: }<17.4 \mathrm{vs.} \geq 17.4 \mathrm{wk} \\
\text { Age: } 9 \mathrm{mo} \\
\text { Assessment Methods: } \\
\text { Parental interview }\end{array}$ & $\begin{array}{l}\text { Limitations: } \\
\text { Cannot determine whether groups } \\
\text { were similar on baseline } \\
\text { characteristics; Cannot determine if } \\
\text { outcome assessors were blinded; } \\
\text { Cannot determine validity/reliability of } \\
\text { outcome assessment; Did not adjust }\end{array}$ \\
\hline
\end{tabular}




\begin{tabular}{|c|c|c|}
\hline $\begin{array}{l}\text { Attrition: } 36 \% \\
\text { Power Analysis and Sufficient } \\
\text { Sample Size: NR } \\
\text { Sex: } \\
50 \% \text { Female } \\
\text { Race/Ethnicity: } \\
88 \% \text { White } \\
3 \% \text { Mixed } \\
2 \% \text { Indian } \\
3 \% \text { Pakistani } \\
0.8 \% \text { Bangladeshi } \\
2 \% \text { Black } \\
1 \% \text { Other } \\
\text { Background Diet: } \\
29 \% \text { never BF; } 42 \% \text { BF }<4 \mathrm{mo} ; \\
29 \% \text { BF } \geq 4 \mathrm{mo}\end{array}$ & $\begin{array}{l}\text { Outcomes: } \\
\text { Weight } \\
\text { Age: } 3-5 y \\
\text { Assessment Methods: } \\
\text { Weight: rapid weight gain, weight measured via Tanita scales } \\
\text { Measured but NR in relation to CFB: Height: stadiometer; } z \text { scores } \\
\text { from measures by British } 1990 \text { standards; Overweight or Obesity by } \\
\text { BMI based on IOTF cut-offs } \\
\text { Confounders accounted for: } \\
\text { Race/ethnicity: X } \\
\text { Gestational age: All full-term } \\
\text { Other: Parental overweight; Smoking; \# children in house }\end{array}$ & $\begin{array}{l}\text { for potential key confounders } \\
\text { (education, SES, sex, maternal age, } \\
\text { race/ethnicity, feeding practices, birth } \\
\text { size, or gestational age) }\end{array}$ \\
\hline 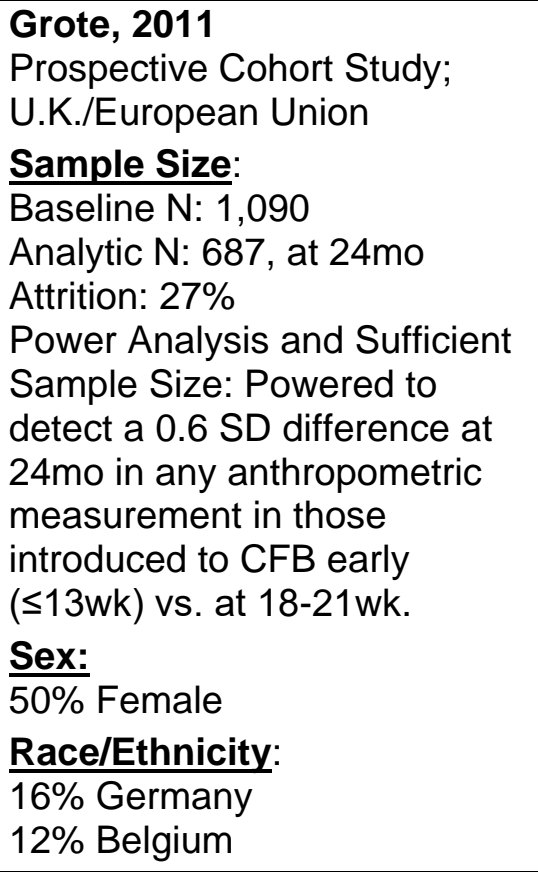 & $\begin{array}{l}\text { Intervention/Exposure: } \\
\text { Age of CFB introduction: } \leq 13,14-17,18-21 \text {, and } \geq 22 \text { wk } \\
\text { Age: } 3,6,9 \text { mo } \\
\text { Assessment Methods: } \\
\text { Food record, 3d, weighed; parental questionnaire } \\
\text { Outcomes: } \\
\text { Body composition; Weight; Length } \\
\text { Age: } 3,6,12,24 \text { mo; } 3-24 m o \\
\text { Assessment Methods: } \\
\text { Body composition: } \\
\text {-WLZ, BMI (kg/cm3), BMIZ based on measures;by WHO standards } \\
\text { Weight, length (via digital scale, recumbent length board, or } \\
\text { stadiometer): used to calculate WAZ/LAZ and weight velocity } \\
\text { (kg/30d or cm/30d) from baseline } \\
\text { Confounders accounted for: } \\
\text { Education: } X \\
\text { Sex: } X \\
\text { Feeding practices: } X \text { (Formula group) }\end{array}$ & $\begin{array}{l}\text { Limitations: } \\
\text { Cannot determine if outcome } \\
\text { assessors were blinded; Cannot } \\
\text { determine validity/reliability of outcome } \\
\text { assessment (lack of detail provided for } \\
\text { single vs. multiple measures); Did not } \\
\text { adjust for potential key confounders } \\
\text { (SES, maternal age, race/ethnicity, or } \\
\text { gestational age) } \\
\text { Article notes types of CFB introduced } \\
\text { may have had a stronger influence } \\
\text { than the timing of CFB on growth } \\
\text { Did not adjust for multiple comparisons }\end{array}$ \\
\hline
\end{tabular}




\begin{tabular}{|c|c|c|}
\hline $\begin{array}{l}24 \% \text { Italy } \\
19 \% \text { Poland } \\
29 \% \text { Spain } \\
\text { Background Diet: } \\
100 \% \text { FF (mixed feeding } \\
\text { allowed <8wk) } \\
\text { Median age of introduction to } \\
\text { CFB } 19 w k ; 7 \% \text { introduced to } \\
\text { CFB } \leq 13 w k ; 97 \% \text { CFB }<6 \text { mo }\end{array}$ & $\begin{array}{l}\text { Other: Country, Birth Order, Age, Partnership Status, Smoking; } \\
\text { Maternal BMI; Baseline infant anthropometric values }\end{array}$ & \\
\hline $\begin{array}{l}\text { Haschke, } 2000 \\
\text { Prospective Cohort Study; } \\
\text { Austria, Germany, Spain, } \\
\text { France, Greece, UK, Hungary, } \\
\text { Croatia, Italy, Ireland, Portugal, } \\
\text { Sweden } \\
\text { Sample Size: } \\
\text { Baseline } N=2,245 \\
\text { Analytic } N=1,071 \\
\text { Attrition = 52\% } \\
\text { Power Analysis and Sufficient } \\
\text { Sample Size: NR } \\
\text { Sex: } \\
48.6 \% \text { Female } \\
\text { Race/Ethnicity: } \\
\text { NR } \\
\text { Background Diet: } \\
15.8 \% \text { WHO group (EBF); } \\
9.1 \% \text { (Early CFB); } 74.9 \% \\
\text { (controls) }\end{array}$ & 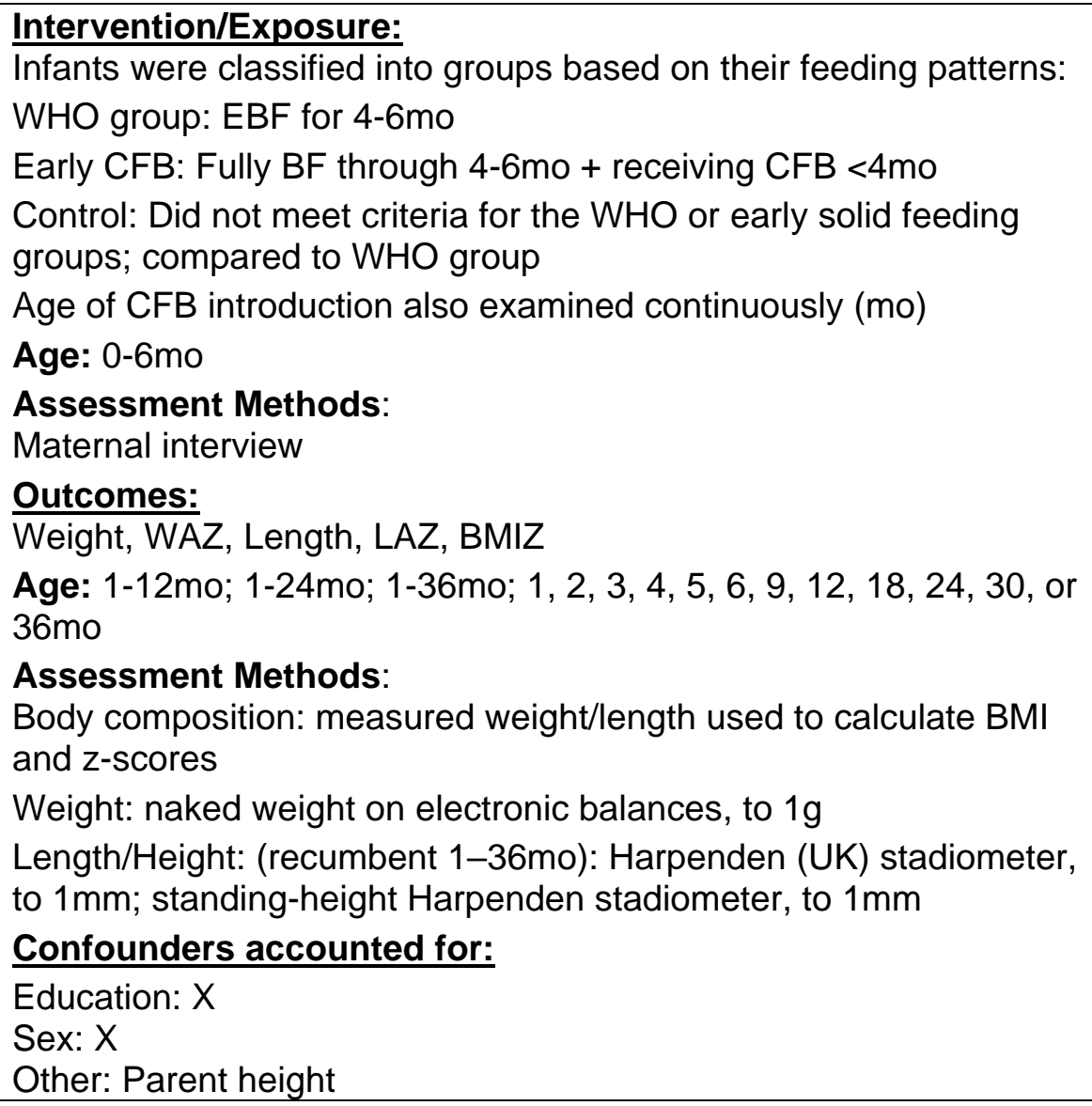 & $\begin{array}{l}\text { Limitations: } \\
\text { Cannot determine whether outcome } \\
\text { assessors were blinded; Cannot } \\
\text { determine validity/reliability of outcome } \\
\text { assessment due to lack of details; Did } \\
\text { not adjust for potential key } \\
\text { confounders (SES, maternal age, } \\
\text { race/ethnicity, feeding practices, birth } \\
\text { size, or gestational age) } \\
\text { Article notes study would have been } \\
\text { strengthened by addition of other } \\
\text { relevant covariates, such as maternal } \\
\text { smoking, family income, etc. }\end{array}$ \\
\hline
\end{tabular}




\begin{tabular}{|c|c|c|}
\hline $\begin{array}{l}\text { Hawkins, 2009 } \\
\text { Prospective Cohort Study; U.K. } \\
\text { (England, Wales, Scotland, } \\
\text { Northern Ireland) } \\
\text { Sample Size: } \\
\text { Baseline N: } 18,296 \\
\text { Analytic N: } 13,188 \\
\text { Attrition: } 27.9 \% \\
\text { Power Analysis and Sufficient } \\
\text { Sample Size: NR } \\
\text { Sex: } \\
50 \% \text { Female } \\
\text { Race/Ethnicity: } \\
7 \% \text { White; } 3 \% \text { Mixed; } 2 \% \\
\text { Indian } \\
3 \% \text { Pakistani; } 0.9 \% \\
\text { Bangladeshi; } 3 \% \text { Black; } 1 \% \\
\text { Other } \\
\text { Background Diet: } \\
30 \% \text { never BF; } 42 \% \text { BF <4mo; } \\
28 \% \text { BF } \geq 4 m o\end{array}$ & $\begin{array}{l}\text { Intervention/Exposure: } \\
\text { Age of CFB introduction: }<4 \text { vs. } \geq 4 \mathrm{mo} \\
\text { Age: } 9 \text { mo } \\
\text { Assessment Methods: } \\
\text { Parental interview } \\
\text { Outcomes: } \\
\text { Weight status } \\
\text { Age: } 0 \text {-3y } \\
\text { Assessment Methods: } \\
\text { Weight status: risk of overweight and obesity, categorized based on } \\
\text { BMI by IOTF cut-offs; BMl calculated from measure weight (Tanita } \\
\text { scales) and height (stadiometer) z scores by British } 1990 \text { standards } \\
\text { Confounders accounted for: } \\
\text { Education: X } \\
\text { SES: X } \\
\text { Sex: X } \\
\text { Maternal age: X } \\
\text { Race/ethnicity: X } \\
\text { Feeding practices: } X \\
\text { Birth size: } X \text { (Birth weight z-scores) } \\
\text { Gestational age: X } \\
\text { Other: Smoking; Parental BMl; Maternal hours; Country; Garden } \\
\text { access; Food access; Parity; Income; Ward; Marital status }\end{array}$ & $\begin{array}{l}\text { Limitations: } \\
\text { Cannot determine whether groups } \\
\text { were similar on baseline } \\
\text { characteristics; Cannot determine if } \\
\text { outcome assessors were blinded; } \\
\text { Cannot determine validity/reliability of } \\
\text { outcome assessment }\end{array}$ \\
\hline $\begin{array}{l}\text { Heinig, } 1993 \\
\text { Prospective Cohort Study; } \\
\text { U.S.; DARLING study } \\
\text { Sample Size: } \\
\text { Baseline N: } 105 \\
\text { Analytic N: } 87 \text { (N: } 46 \text { BF; N: } 41 \\
\text { FF at 9mo) } \\
\text { Attrition: 17\% } \\
\text { Power Analysis and Sufficient } \\
\text { Sample Size: NR (N: } 56 \text { per } \\
\text { group Dewey, 1992) }\end{array}$ & $\begin{array}{l}\text { Intervention/Exposure: } \\
\text { Age of CFB introduction: BF: }<26 \text { vs. } \geq 26 w k ; F F: \text { continuously and } \\
<20 \text { vs. } \geq 20 w k \\
\text { CFB: Date when first CFB offered was accepted } \\
\text { Age: } 0-26 w k(\sim 0-6 \mathrm{mo}) \\
\text { Assessment Methods: } \\
\text { Food record,4d, weighed } \\
\text { Outcomes: } \\
\text { Weight; Length; Body composition } \\
\text { Age: birth-4mo, 4-6mo, 6-9mo, 9-12mo; 1-18mo }\end{array}$ & $\begin{array}{l}\text { Limitations: } \\
\text { Cannot determine if outcome } \\
\text { assessors were blinded; Cannot } \\
\text { determine validity/reliability of outcome } \\
\text { assessment; Did adjust for potential } \\
\text { key confounders (gestational age) } \\
\text { Results were partially reported for FF } \\
\text { infants by age of introduction to CFB in } \\
\text { text (results/discussion) but data not } \\
\text { shown } \\
\text { Limited generalizability due to small } \\
\text { geographic region }\end{array}$ \\
\hline
\end{tabular}




\begin{tabular}{|c|c|c|}
\hline $\begin{array}{l}\text { Sex: } \\
52.8 \% \text { Female } \\
\text { Race/Ethnicity: } \\
87 \% \text { White, non-Hispanic } \\
\text { Background Diet: } \\
\text { All infants were BF or FF and } \\
\text { were categorized into groups } \\
\text { based on when the child was } \\
\text { introduced to solids } \\
\text { Mean age of CFB introduction } \\
\text { was later in FF vs. BF infants } \\
\text { (23wk vs. } 20 \text { wk) }\end{array}$ & $\begin{array}{l}\text { Assessment Methods: } \\
\text { Body composition: WLZ scores by NCHS reference were calcuated } \\
\text { from measured weight/height } \\
\text { Weight: velocity(g/month) of mean gain calcuated from measured } \\
\text { weight; WAZ } \\
\text { Length: velocity (cm/month) of mean gain calcuated from measured } \\
\text { height; LAZ } \\
\text { Confounders accounted for: } \\
\text { Sex: X } \\
\text { Feeding practices: X } \\
\text { Birth size: X (Based on stratified matching of birth weight) } \\
\text { Gestational age: All full-term } \\
\text { Other: Growth from 0-4mo; protein intake; energy intake; Stratified } \\
\text { matching was used to account for education, SES, maternal age, } \\
\text { race/ethnicity }\end{array}$ & \\
\hline $\begin{array}{l}\text { Huh, } 2011 \\
\text { Prospective Cohort Study; U.S. } \\
\text { Sample Size: } \\
\text { Baseline N: } 1579 \\
\text { Analytic N: } 847 \\
\text { Attrition: } 46 \% \\
\text { Power Analysis and Sufficient } \\
\text { Sample Size: NR } \\
\text { Sex: } \\
50.3 \% \text { Female } \\
\text { Race/Ethnicity: } \\
29.3 \% \text { non-white } \\
\text { Background Diet: } \\
67 \% \text { BF; 33\% FF at } 4 \mathrm{mo}\end{array}$ & $\begin{array}{l}\text { Intervention/Exposure: } \\
\text { Age of CFB introduction: }<4,4-5 \text {, and } \geq 6 \mathrm{mo} \text { in FF and in BF infants } \\
\text { CFB: } 10 \text { solids/food groups, including infant cereal, other starches } \\
\text { (teething biscuits, crackers), fruit, vegetables, meat, } \\
\text { chicken or turkey, peanut butter, other cow's milk dairy product } \\
\text { Age: } 0-6 m o \\
\text { Assessment Methods: } \\
\text { Maternal questionnaire } \\
\text { Outcomes: } \\
\text { Weight status, Body composition (Adiposity); Weight; Height; } \\
\text { Age: } 3 y \\
\text { Assessment Methods: } \\
\text { Weight status: BMl calculated from measured weight/height; BMI z; } \\
\text { used for obesity=BMI } \geq 95 \mathrm{th} \% \text { tile } \\
\text { Body composition: skinfold thickness via calipers; BMI and BMIZ } \\
\text { from measures } \\
\text { Weight: Seca scale to } 0.1 \mathrm{~kg} \\
\text { Height: stadiometer to } 0.1 \mathrm{~cm}\end{array}$ & $\begin{array}{l}\text { Limitations: } \\
\text { Groups differed on baseline } \\
\text { characteristics and differences were } \\
\text { not adjusted for in analyses; Cannot } \\
\text { determine if outcome assessors were } \\
\text { blinded; Cannot determine } \\
\text { reliability/validity of outcome } \\
\text { assessment; Did not adjust for } \\
\text { potential key confounders (maternal } \\
\text { age) } \\
\text { Intake regulation may differ in FF vs. } \\
\text { BF infants, leading to greater intake of } \\
\text { CFB; Wider Cl reflecting small number } \\
\text { of infants in CFB>6mo group } \\
\text { ( } n=25 / 279 \text { ); Limited generalizability }\end{array}$ \\
\hline
\end{tabular}




\begin{tabular}{|c|c|c|}
\hline & $\begin{array}{l}\text { Confounders accounted for: } \\
\text { Education: } X \\
\text { SES: } X \\
\text { Sex: X } \\
\text { Race/ethnicity: X } \\
\text { Birth size: X (Birth weight, birth weight-for-gestational age z-score) } \\
\text { (Excluded, no effect) } \\
\text { Gestational age: (Excluded, no effect) } \\
\text { Other: Early infant-growth 0-4mo; Maternal and Paternal BMI }\end{array}$ & \\
\hline $\begin{array}{l}\text { Imai, } 2014 \\
\text { Prospective Cohort Study; } \\
\text { Iceland } \\
\text { Sample Size: } \\
\text { Baseline N: } 250 \\
\text { Analytic N: } 154 \\
\text { Attrition: } 38.4 \% \\
\text { Power Analysis and Sufficient } \\
\text { Sample Size: NR } \\
\text { Sex: } \\
51 \% \text { Female } \\
\text { Race/Ethnicity: } \\
\text { NR } \\
\text { Background Diet: } \\
40 \% \text { EBF; } 7.7 \% \text { EFF at } 5 \mathrm{mo}\end{array}$ & $\begin{array}{l}\text { Intervention/Exposure: } \\
\text { Age of CFB introduction: at } 5 \mathrm{mo} \\
\text { CFB: EBF (N: 62), CFB+BF (N: } 57) \\
\text { Age: } 5-8 m o \\
\text { Assessment Methods: } \\
\text { Caregiver questionnaire; } 24 \mathrm{~h} \text { recall; Food record, weighed 3d } \\
\text { Outcomes: } \\
\text { Weight; Length; Body compsotion } \\
\text { Age: } 6-12 \mathrm{mo} ; 12-18 \mathrm{mo}, 6 \mathrm{y} \\
\text { Assessment Methods: } \\
\text { Body composition: BMI from measured height and weight } \\
\text { Weight: scale to 0.1kg; reported as absolute and change in; used for } \\
\text { BMl } \\
\text { Length: stadiometer to 0.5cm; reported as absolute and change in; } \\
\text { used for BMI } \\
\text { Confounders accounted for: } \\
\text { Education: } X \\
\text { Sex: } X \\
\text { Feeding practices: } X \text { (in model) } \\
\text { Birth size: } X \text { (Birth weight) }\end{array}$ & $\begin{array}{l}\text { Limitations: } \\
\text { Cannot determine if outcome } \\
\text { assessors were blinded; Cannot } \\
\text { determine validity/reliability of outcome } \\
\text { assessment; Did not adjust for } \\
\text { potential confounders (SES, maternal } \\
\text { age, race/ethnicity, or gestational age); } \\
\text { Did not account for high attrition rate } \\
\text { (38\%) } \\
\text { Small sample size (insufficient power); } \\
\text { Did not collect information on exact } \\
\text { time of introduction of CFB }\end{array}$ \\
\hline $\begin{array}{l}\text { Johnson, } 2014 \\
\text { Prospective Cohort Study; U.K. } \\
\text { Sample Size: } \\
\text { Baseline N: } 4860\end{array}$ & $\begin{array}{l}\text { Intervention/Exposure: } \\
\text { Age of CFB introduction: }<4 \mathrm{mo}(\mathrm{n}=1714), \sim 5 \mathrm{mo}(\mathrm{n}=1667), \geq 6 \mathrm{mo} \\
(\mathrm{n}=1364) \\
\text { Age: } 8.2 \mathrm{mo}\end{array}$ & $\begin{array}{l}\text { Limitations: } \\
\text { Cannot determine validity/reliability of } \\
\text { outcome assessment (parent report); } \\
\text { Outcome assessors not blind; Did not }\end{array}$ \\
\hline
\end{tabular}




\begin{tabular}{|c|c|c|}
\hline $\begin{array}{l}\text { Analytic N: } 4251 \\
\text { Attrition: } 12.5 \% \\
\text { Power Analysis and Sufficient } \\
\text { Sample Size: NR } \\
\text { Sex: } \\
50.3 \% \text { Female } \\
\text { Race/Ethnicity: } \\
\text { NR } \\
\text { Background Diet: } \\
23 \% \text { never BF, } n=1097 ; 53 \% \\
\text { BF birth to } 4 \text { mo, } n=2507 ; 24 \% \\
\text { BF > 4 mo, } n=1168 ; \sim 2 \% \text { EBF } \\
\geq 4 \text { mo, n=95 }\end{array}$ & $\begin{array}{l}\text { Assessment Methods: } \\
\text { Caregiver questionnaire } \\
\text { Outcomes: } \\
\text { Weight } \\
\text { Age: } 6 \text { mo, } 0-6 \text { mo } \\
\text { Assessment Methods: } \\
\text { Weight: Parent report of child health record weight: SDS according } \\
\text { to reported weight; SITAR weight trajectory: Tempo was the age of } \\
\text { peak weight velocity in wk (higher=delayed tempo); Weight velocity } \\
\text { (kg/wk) } \\
\text { Confounders accounted for: } \\
\text { Education: X } \\
\text { SES: X } \\
\text { Sex: } X \\
\text { Maternal age: X } \\
\text { Feeding practices: } X \\
\text { Gestational age: } X \\
\text { Other: Birth order; parity; occupation; smoking; zygosity; child age at } \\
\text { baseline }\end{array}$ & $\begin{array}{l}\text { adjust for potential confounders (birth } \\
\text { size, race/ethnicity) } \\
\text { Groups unbalanced ( } 29 \% \text { in CFB } \\
>6 \text { mo group) }\end{array}$ \\
\hline $\begin{array}{l}\text { Kalies, } 2005 \\
\text { Prospective Cohort Study; } \\
\text { Germany } \\
\text { Sample Size: } \\
\text { Baseline } N=3,036 \\
\text { Analytic } N=2,337 \\
\text { Attrition }=23 \% \\
\text { Power Analysis and Sufficient } \\
\text { Sample Size: NR } \\
\text { Sex: } \\
49 \% \text { Female } \\
\text { Race/Ethnicity: } \\
\text { NR } \\
\text { Background Diet: } \\
\text { Feeding mode during time of } \\
\text { observation: } 24.4 \% \text { EBF first } 0 \text { - }\end{array}$ & $\begin{array}{l}\text { Intervention/Exposure: } \\
\text { Age of CFB introduction: } 1-3 \mathrm{mo}, 4-6 \mathrm{mo},>6 \mathrm{mo} \\
\text { Age: } 2 \mathrm{y} \\
\text { Assessment Methods: } \\
\text { Caregiver questionnaire } \\
\text { Outcomes: } \\
\text { Weight } \\
\text { Age: } 24 \mathrm{mo} \\
\text { Assessment Methods: } \\
\text { Weight, height: recorded at preventive medical check-ups; reported } \\
\text { as risk of elevated weight gain at } 24 \mathrm{mo} \\
\text { Confounders accounted for: } \\
\text { SES: } X \\
\text { Sex: } X \\
\text { Feeding practices: } X\end{array}$ & $\begin{array}{l}\text { Limitations: } \\
\text { Cannot determine whether groups } \\
\text { were similar on baseline } \\
\text { characteristics; Cannot determine if } \\
\text { outcome assessors were blinded; } \\
\text { Cannot determine validity/reliability of } \\
\text { outcome assessment (height and } \\
\text { weight non-duplicate at medical check- } \\
\text { ups); Did not account for high attrition } \\
\text { rate (23\%); Did not adjust for potential } \\
\text { key confounders (education; maternal } \\
\text { age; race/ethnicity, birth size or } \\
\text { gestational age) } \\
\text { Parent self-reported feeding data; } \\
\text { primary objective of study was not } \\
\text { timing/type of CFB as such it appears }\end{array}$ \\
\hline
\end{tabular}




\begin{tabular}{|c|c|c|}
\hline $\begin{array}{l}1 \mathrm{mo}, 16.3 \% \text { BF for } 2-3 \mathrm{mo}, \\
20.8 \% \mathrm{BF} \text { for } 4-5 \mathrm{mo}, 38.6 \% \mathrm{BF} \\
>\text { or }=6 \mathrm{mo} .\end{array}$ & Other: Smoking; maternal BMI; birth order; study center; All full-term & $\begin{array}{l}\text { IV/Exposure was used as a } \\
\text { confounding in models }\end{array}$ \\
\hline $\begin{array}{l}\text { Khadivzadeh, } 2004 \\
\text { Prospective Cohort Study; Iran } \\
\text { Sample Size: } \\
\text { Baseline } N=200 \\
\text { Analytic } N=193 \\
\text { Attrition }=3.5 \% \\
\text { Power Analysis and Sufficient } \\
\text { Sample Size: NR } \\
\text { Sex: } \\
\text { 47\% Female } \\
\text { Race/Ethnicity: } \\
\text { NR } \\
\text { Background Diet: } \\
100 \% \text { BF at 4mo }\end{array}$ & $\begin{array}{l}\text { Intervention/Exposure: } \\
\text { Age of CFB introduction: CFB+BF at } 4 \mathrm{mo} \text { vs EBF 4-6mo } \\
\text { Age: } 4.5 \mathrm{mo} \\
\text { Assessment Methods: } \\
\text { Maternal interview } \\
\text { Outcomes: } \\
\text { Weight; Length } \\
\text { Age: } 5,6 \text { mo; } 4-6 \text { mo } \\
\text { Assessment Methods: } \\
\text { Weight: Digital scale; weight and weight gain } \\
\text { Length: Recumbent board; length and length gain } \\
\text { Confounders accounted for: } \\
\text { Sex: } X \\
\text { Maternal age: } X \\
\text { Race/ethnicity: } X\end{array}$ & $\begin{array}{l}\text { Limitations: } \\
\text { Cannot determine whether groups } \\
\text { were similar on baseline } \\
\text { characteristics; Cannot determine if } \\
\text { outcome assessors were blinded; } \\
\text { Cannot determine validity/reliability of } \\
\text { outcome assessment; Did not adjust } \\
\text { for potential key confounders } \\
\text { (education, SES, birth size, gestational } \\
\text { age, feeding practices) } \\
\text { Degree of antenatal care was } \\
\text { significantly higher in exclusively BF vs } \\
\text { BF + CFB group but not included as } \\
\text { confounder in analysis. }\end{array}$ \\
\hline $\begin{array}{l}\text { Kramer, } 1985 \\
\text { Prospective Cohort Study; } \\
\text { Canada } \\
\text { Sample Size: } \\
\text { Baseline N: } 462 \\
\text { Analytic N: } 347 \\
\text { Attrition: } 25 \% \\
\text { Power Analysis and Sufficient } \\
\text { Sample Size: NR } \\
\text { Sex: } \\
46.1 \% \text { Female } \\
\text { Race/Ethnicity: } \\
\text { NR } \\
\text { Background Diet: } \\
\text { At birth, } 58.4 \% \text { EBF, } 72.6 \%\end{array}$ & $\begin{array}{l}\text { Intervention/Exposure: } \\
\text { Age of CFB introduction: Continuous } \\
\text { CFB: cereals and other solids } \\
\text { Age: birth-1y } \\
\text { Assessment Methods: } \\
\text { Maternal interview } \\
\text { Outcomes: } \\
\text { Weight; Body composition } \\
\text { Age: } 24 \text { mo } \\
\text { Assessment Methods: } \\
\text { Body composition: } \\
\text { - BMI: calculated via weight/height2 } \\
\text { - Skinfold thickness: sum of triceps, subbscapular, and suprailiac } \\
\text { measures via calipers by research staff }\end{array}$ & $\begin{array}{l}\text { Limitations: } \\
\text { Cannot determine whether groups } \\
\text { were similar on baseline } \\
\text { characteristics; Cannot determine } \\
\text { validity/reliability of outcome } \\
\text { assessment (insufficient detail); Did not } \\
\text { account for high rate of attrition (>20\%) } \\
\text {; Did not adjust for potential key } \\
\text { confounders (race/ethnicity, birth size, } \\
\text { gestational age) }\end{array}$ \\
\hline
\end{tabular}




\begin{tabular}{|c|c|c|}
\hline $\begin{array}{l}\text { Any BF; At } 4 \mathrm{mo}, 18.8 \% \text { EBF, } \\
37.2 \% \text { Any BF; At } 6 \mathrm{mo}, 10.4 \% \\
\text { EBF, } 22.5 \% \text { Any BF (Kramer, } \\
1985 \mathrm{~b}, \text { based on n: } 382 \text { at } \\
6 \mathrm{mo})\end{array}$ & $\begin{array}{l}\text { Weight: measured at home-visit by research staff } \\
\text { Confounders accounted for: } \\
\text { Education: } X \\
\text { SES: } X \\
\text { Sex: X } \\
\text { Maternal age: } X \\
\text { Feeding practices: } X \text { (duration of BF, step-wise) } \\
\text { Gestational age: All full-term }\end{array}$ & \\
\hline $\begin{array}{l}\text { Kramer, 1985b } \\
\text { Prospective Cohort Study; } \\
\text { Canada } \\
\text { Sample Size: } \\
\text { Baseline N: } 462 \\
\text { Analytic N: } 382 \\
\text { Attrition: } 17 \% \\
\text { Power Analysis and Sufficient } \\
\text { Sample Size: NR } \\
\text { Sex: } \\
46.1 \% \text { Female } \\
\text { Race/Ethnicity: } \\
\text { NR } \\
\text { Background Diet: } \\
\text { At birth, 58.4\% EBF, 72.6\% } \\
\text { Any BF; At 4mo, 18.8\% EBF, } \\
37.2 \% \text { Any BF; At } 6 m 0,10.4 \% \\
\text { EBF, 22.5\% Any BF }\end{array}$ & $\begin{array}{l}\text { Intervention/Exposure: } \\
\text { Age of CFB introduction: Continuous } \\
\text { CFB: cereals and other solids } \\
\text { Age: } 0-4 \text { mo } \\
\text { Assessment Methods: } \\
\text { Maternal interview } \\
\text { Outcomes: } \\
\text { Body composition; Weight } \\
\text { Age: } 6 \text { mo, } 12 \text { mo } \\
\text { Assessment Methods: } \\
\text { Body composition: } \\
\text { - BMI: calculated via weight/height2 } \\
\text { - Skinfold thickness: sum of triceps, subbscapular, and suprailiac } \\
\text { measures via calipers by research staff } \\
\text { Weight: measured at home-visit by research staff } \\
\text { Confounders accounted for: } \\
\text { Education: } X \\
\text { SES: } X \\
\text { Sex: } X \\
\text { Maternal age: } X \\
\text { Feeding practices: } X \\
\text { Birth size: } X \text { (birth weight) } \\
\text { Gestational age: All full-term }\end{array}$ & $\begin{array}{l}\text { Limitations: } \\
\text { Cannot determine whether groups } \\
\text { were similar on baseline } \\
\text { characteristics; Cannot determine } \\
\text { validity/reliability of outcome } \\
\text { assessment (insufficient detail); Did not } \\
\text { adjust for potential key confounders } \\
\text { (race/ethnicity, birth size, gestational } \\
\text { age) } \\
\text { Lack of detail on outcome } \\
\text { measurements }\end{array}$ \\
\hline
\end{tabular}




\begin{tabular}{|c|c|c|}
\hline $\begin{array}{l}\text { Kramer, 2004 } \\
\text { Prospective Cohort Study; } \\
\text { Belarus } \\
\text { Sample Size: } \\
\text { Baseline N: } 17,601 \\
\text { Analytic N: } 17,046 \\
\text { Attrition: } 3 \% \\
\text { Power Analysis and Sufficient } \\
\text { Sample Size: NR } \\
\text { Sex: } \\
\text { NR } \\
\text { Race/Ethnicity: } \\
\text { NR } \\
\text { Background Diet: } \\
\text { NR; } 100 \% \text { were BF at birth }\end{array}$ & $\begin{array}{l}\text { Intervention/Exposure: } \\
\text { Age of CFB introduction: intake (any vs. none) of CFB at the start of } \\
\text { the respective growth interval } \\
\text { CFB: cereals, other solids, and juices } \\
\text { Age: } 0-12 \text { mo } \\
\text { Assessment Methods: } \\
\text { Maternal interview } \\
\text { Outcomes: } \\
\text { Body composition; Weight; Length; HC } \\
\text { Age: } 1 \text {-3mo; 3-6mo; } 6-9 \text { mo; } 9-12 \mathrm{mo} \\
\text { Assessment Methods: } \\
\text { Body composition: WLZ based on CDC/WHO, } 2000 \\
\text { Weight: NR; used to calculate WAZ } \\
\text { Length: NR; used to calculate LAZ } \\
\text { HC: NR } \\
\text { Confounders accounted for: } \\
\text { Education: X } \\
\text { Birth size: X (All with birth weight }>2.5 \mathrm{~kg} \text { ) } \\
\text { Gestational age: All full-term } \\
\text { Other: Anthropometric outcome at current time (WAZ, LAZ, WLZ, } \\
\text { HC), geographic region, urbanicity, hospital }\end{array}$ & $\begin{array}{l}\text { Limitations: } \\
\text { Cannot determine whether groups } \\
\text { were similar on baseline } \\
\text { characteristics; Cannot determine } \\
\text { whether outcome assessors were } \\
\text { blinded; Cannot determine } \\
\text { validity/reliability of outcome } \\
\text { assessment (unstandardardized); Did } \\
\text { not adjust for potential key } \\
\text { confounders (SES, sex, maternal age, } \\
\text { race/ethnicity though participants were } \\
\text { from RCT) } \\
\text { Cereals consumed may not be } \\
\text { generalizable; Only } 1.2 \% \text { of infants } \\
\text { were consuming cereals at } 3 \text { mo; } \\
\text { Cannot rule out reverse causality, } \\
\text { residual confounding, and selection } \\
\text { bias; Trial promoted breast-feeding; } \\
\text { Outcome assessment was } \\
\text { unstandardized (various methods } \\
\text { used; not primary outcome of original } \\
\text { RCT) }\end{array}$ \\
\hline $\begin{array}{l}\text { Kuperberg, } 2006 \\
\text { Prospective Cohort Study; } \\
\text { Canada } \\
\text { Sample Size: } \\
\text { Baseline N: } 102 \\
\text { Analytic N: } 71 \\
\text { Attrition: } 30 \% \\
\text { Power Analysis and Sufficient } \\
\text { Sample Size: NR } \\
\text { Sex: } \\
49 \% \text { Female } \\
\text { Race/Ethnicity: } \\
\text { NR }\end{array}$ & $\begin{array}{l}\text { Intervention/Exposure: } \\
\text { Age of CFB introduction: Continuous; Dichotomous }(\mathrm{y} / \mathrm{n})<4 \mathrm{mo} \\
\text { CFB: solids, and cow's milk } \\
\text { Age: } 48 \mathrm{mo} \\
\text { Assessment Methods: } \\
\text { Maternal questionnaire; } 24 \mathrm{hr} \text { recall } \\
\text { Outcomes: } \\
\text { Weight status } \\
\text { Age: } 48 \mathrm{mo} \\
\text { Assessment Methods: } \\
\text { Weight status: risk of overweight, based on } \mathrm{BMI} \geq 85 \mathrm{th} \% \text { tile from } \\
\text { measured length/height and weight. Weight at } 3 \text { and } 18 \mathrm{mo} \\
\text { measured via portable infant scale; at } 33 \text { and } 48 \mathrm{mo} \text {, electronic scale. }\end{array}$ & $\begin{array}{l}\text { Limitations: } \\
\text { Cannot determine whether groups } \\
\text { were similar on baseline } \\
\text { characteristics; Cannot determine } \\
\text { whether outcome assessors were } \\
\text { blinded; Did not account for high rate } \\
\text { of attrition to 48mo; Did not adjust for } \\
\text { potential key confounders (education, } \\
\text { SES, sex, maternal age, race/ethnicity, } \\
\text { feeding practices, birth size, or } \\
\text { gestational age) } \\
\text { Mother's health information not } \\
\text { available at 3mo; Limited IV/Exposure }\end{array}$ \\
\hline
\end{tabular}




\begin{tabular}{|c|c|c|}
\hline $\begin{array}{l}\text { Background Diet: } \\
75 \% \text { initiated } \mathrm{BF} \\
57.1 \% \text { CFB by } 4 \mathrm{mo}\end{array}$ & $\begin{array}{l}\text { Length: at } 3 \text { and } 18 \text { mo measured via pediatric length board; height } \\
\text { at } 33 \text { and } 48 \mathrm{mo} \text {, measured via microtoise modified tape measure } \\
\text { Confounders accounted for: } \\
\text { Other: NR }\end{array}$ & $\begin{array}{l}\text { assessment to maternal self-report and } \\
\text { one, } 24 \mathrm{hr} \text { recall; Lack of relevant data }\end{array}$ \\
\hline $\begin{array}{l}\text { Lande, } 2005 \\
\text { Prospective Cohort Study; } \\
\text { Norway } \\
\text { Sample Size: } \\
\text { Baseline N: } 1934 \text { (3000 mailed; } \\
1934 \text { returned at } 12 \mathrm{mo}) \\
\text { Analytic N: } 1441 \\
\text { Attrition: } 25 \% \\
\text { Power Analysis and Sufficient } \\
\text { Sample Size: NR } \\
\text { Sex: } \\
47 \% \text { Female } \\
\text { Race/Ethnicity: } \\
\text { NR } \\
\text { Background Diet: } \\
\text { NR }\end{array}$ & $\begin{array}{l}\text { Intervention/Exposure: } \\
\text { Age of CFB introduction: } \leq 4,4-5,>5 \mathrm{mo} \\
\text { Age: } 6 \mathrm{mo} ; 12 \mathrm{mo} \\
\text { Assessment Methods: } \\
\text { Parental FFQ } \\
\text { Outcomes: } \\
\text { Body composition } \\
\text { Age: } 12 \mathrm{mo} \\
\text { Assessment Methods: } \\
\text { Body composition: } \\
\text { - BMl: calculated based on height and weight measured by health- } \\
\text { care personnel } \\
\text { Confounders accounted for: } \\
\text { Education: } X \\
\text { SES: } X \\
\text { Sex: } X \\
\text { Maternal age: } X \\
\text { Feeding practices: } X \\
\text { Birth size: } X \\
\text { Gestational age: All full-term } \\
\text { Other: Smoking; Birth order }\end{array}$ & $\begin{array}{l}\text { Limitations: } \\
\text { Cannot determine whether groups } \\
\text { were similar on baseline } \\
\text { characteristics; Cannot determine } \\
\text { whether outcome assessors were } \\
\text { blinded; Cannot determine } \\
\text { validity/reliability of outcome } \\
\text { assessment; Cannot determine if } \\
\text { length of follow-up differed between } \\
\text { groups; Did not adjust analyses for } \\
\text { potential key confounders } \\
\text { (race/ethnicity, and gestational age) } \\
\text { Not all relevant data was } \\
\text { shown/reported; } \\
\text { Note: daily/weekly use of CFB at } 6 \text { mo } \\
\text { was collected but only compared } \\
\text { against PI at birth; or in univariate } \\
\text { analyses; and not against BMl at } \\
12 \text { mo; univariate analyses showed } \\
\text { significant effect of age of CFB intro }\end{array}$ \\
\hline $\begin{array}{l}\text { Lauver, } 1981 \\
\text { Prospective Cohort Study; } \\
\text { United States } \\
\text { Sample Size: } \\
\text { Baseline N: } 202 \\
\text { Analytic N: } 77 \\
\text { Attrition: } 62 \% \\
\text { Power Analysis and Sufficient } \\
\text { Sample Size: NR }\end{array}$ & $\begin{array}{l}\text { Intervention/Exposure: } \\
\text { Age of CFB introduction: } \leq 5 \text { or }>5 \mathrm{mo} \\
\text { CFB: Beikost/solids; Non-compliant were given CFB } \leq 5 \mathrm{mo} \text {; } \\
\text { compliant were given CFB }>5 \mathrm{mo} \\
\text { Age: } 5 \mathrm{mo} \\
\text { Assessment Methods: } \\
\text { Caregiver interview }\end{array}$ & $\begin{array}{l}\text { Limitations: } \\
\text { Cannot determine whether groups } \\
\text { were similar on baseline } \\
\text { characteristics; Cannot determine } \\
\text { adherence to protocols across groups; } \\
\text { Unintended exposures not accounted } \\
\text { for (differences between groups in BF } \\
\text { and cow's milk); Cannot determine } \\
\text { whether investigators were blinded; }\end{array}$ \\
\hline
\end{tabular}




\begin{tabular}{|c|c|c|}
\hline $\begin{array}{l}\text { Sex: } \\
\text { NR } \\
\text { Race/Ethnicity: } \\
47 \% \text { Black } \\
48 \% \text { White } \\
5 \% \text { Other } \\
\text { Background Diet: } \\
25 \% \text { BF; } 75 \% \text { formula; } \\
12 \% \text { consumed CM by } 6 \text { mo }\end{array}$ & $\begin{array}{l}\text { Outcomes: } \\
\text { Weight; Length } \\
\text { Age: } 6 \text { mo } \\
\text { Assessment Methods: } \\
\text { Weight and length recorded at clinic visits } \\
\text { Confounders accounted for: } \\
\text { Maternal age: } X \\
\text { Feeding practices: } X \\
\text { Birth size: } X\end{array}$ & $\begin{array}{l}\text { Cannot determine validity/reliability of } \\
\text { outcome assessment; Did not adjust } \\
\text { for potential key confounders } \\
\text { (education, SES, sex, or gestational } \\
\text { age); Adequacy of statistical methods } \\
\text { was insufficient }\end{array}$ \\
\hline $\begin{array}{l}\text { Layte, } 2014 \\
\text { Prospective Cohort Study; } \\
\text { Ireland } \\
\text { Sample Size: } \\
\text { Baseline N: } 16,136 \\
\text { Analytic N: } 11,134 \\
\text { Attrition: } 31 \% \\
\text { Power Analysis and Sufficient } \\
\text { Sample Size: NR } \\
\text { Sex: } \\
49 \% \text { Female } \\
\text { Race/Ethnicity: } \\
86.3 \% \text { Irish; } 1.8 \% \text { UK; } \\
6.6 \% \text { EU } \\
2.4 \% \text { African } \\
2.8 \% \text { Other } \\
\text { Background Diet: } \\
57.1 \% \text { never BF; } 4.3 \% \\
\text { BF<3mo; } 5.6 \% \text { BF<6mo; } 8.8 \% \\
\text { BF 6+mo }\end{array}$ & $\begin{array}{l}\text { Intervention/Exposure: } \\
\text { Age of CFB introduction: }<4 \mathrm{mo},<5 \mathrm{mo},<6 \mathrm{mo}, \geq 6 \mathrm{mo} \\
\text { Age: } 9 \mathrm{mo} \\
\text { Assessment Methods: } \\
\text { Maternal interview } \\
\text { Outcomes: } \\
\text { Weight status; Weight } \\
\text { Age: } 0-9 \mathrm{mo}, 3 y \\
\text { Assessment Methods: } \\
\text { Weight status: risk of obesity based on BMI , calculated from } \\
\text { measured height/weight by IOTF thresholds } \\
\text { Weight: rapid gain calculated by measured weight on Seca scale to } \\
0.5 \mathrm{~kg} \text { and length/height measured by height stick and indexed by } \\
\text { IOTF } \\
\text { Confounders accounted for: } \\
\text { SES: } X \\
\text { Sex: } X \\
\text { Maternal age: } X \\
\text { Feeding practices: } X \text { (BF in model) } \\
\text { Birth size: } X \text { (Birth weight) } \\
\text { Gestational age: } X \\
\text { Other: Multiples; Birth order; Weight gain in pregnancy }\end{array}$ & $\begin{array}{l}\text { Limitations: } \\
\text { Cannot determine whether groups } \\
\text { were similar on baseline } \\
\text { characteristics; Cannot determine } \\
\text { whether outcome assessors were } \\
\text { blinded; Cannot determine } \\
\text { validity/reliability of outcome } \\
\text { assessment; Did not adjust analyses } \\
\text { for potential key confounders } \\
\text { (education, race/ethnicity, and feeding } \\
\text { practices though part of modeling) }\end{array}$ \\
\hline
\end{tabular}




\begin{tabular}{|c|c|c|}
\hline $\begin{array}{l}\text { Leary, } 2015 \\
\text { Prospective Cohort Study; } \\
\text { United Kingdom } \\
\text { Sample Size: } \\
\text { Baseline N: } 5,112 \\
\text { Analytic N: } 4,750 \\
\text { Attrition: } 7 \% \\
\text { Power Analysis and Sufficient } \\
\text { Sample Size: NR } \\
\text { Sex: } \\
52.3 \% \text { Female } \\
\text { Race/Ethnicity: } \\
\text { NR } \\
\text { Background Diet: } \\
36.4 \% \text { EBF; 49.5\% PBF; } \\
\text { 14.1\% no BF }\end{array}$ & $\begin{array}{l}\text { Intervention/Exposure: } \\
\text { Age of CFB introduction: } \leq 2,3, \geq 4 \mathrm{mo} \\
\text { Age: } 6 \text { mo } \\
\text { Assessment Methods: } \\
\text { Maternal questionnaire } \\
\text { Outcomes: } \\
\text { Body composition } \\
\text { Age: } 15 y \\
\text { Assessment Methods: } \\
\text { Body composition: FM, lean mass via DXA; BMI: not reported in } \\
\text { relation to CFB } \\
\text { Confounders accounted for: } \\
\text { Education: } X \\
\text { SES: } X \\
\text { Sex: } X \\
\text { Maternal age: } X \\
\text { Feeding practices: } X \\
\text { Birth size: } X \\
\text { Gestational age: } X \\
\text { Other: Current age; Maternal height; Parity }\end{array}$ & $\begin{array}{l}\text { Limitations: } \\
\text { Cannot determine whether groups } \\
\text { were similar on baseline } \\
\text { characteristics; Did not adjust for } \\
\text { potential key confounders } \\
\text { (race/ethnicity) }\end{array}$ \\
\hline $\begin{array}{l}\text { Makela, 2014 } \\
\text { Prospective Cohort Study; } \\
\text { Finland } \\
\text { Sample Size: } \\
\text { Baseline N: } 1797 \\
\text { Analytic N: } 848 \\
\text { Attrition: } 52 \% \\
\text { Power Analysis and Sufficient } \\
\text { Sample Size: NR } \\
\text { Sex: } \\
\text { 48\% Female } \\
\text { Race/Ethnicity: } \\
\text { NR }\end{array}$ & $\begin{array}{l}\text { Intervention/Exposure: } \\
\text { Age of CFB introduction: Continuous, mo; Categorical }<4,4-6,>6 \mathrm{mo} \\
\text { CFB: vegetables, fruits, grains, meat, fish and dairy categories not } \\
\text { defined } \\
\text { Age: birth-12mo } \\
\text { Assessment Methods: } \\
\text { Food diaries, collected in real-time } \\
\text { Outcomes: } \\
\text { Weight status; Weight } \\
\text { Age: birth-24mo; at } 24 \mathrm{mo} \\
\text { Assessment Methods: } \\
\text { Weight status: risk of overweight or obesity based on Finnish age- } \\
\text { and sex-specific growth references }\end{array}$ & $\begin{array}{l}\text { Limitations: } \\
\text { Cannot determine whether groups } \\
\text { were similar at baseline on key } \\
\text { characteristics; Cannot determine } \\
\text { whether outcome assessors were } \\
\text { blinded; Cannot determine the } \\
\text { validity/reliability of outcome } \\
\text { assessment (i.e., single vs multiple } \\
\text { growth measures); Cannot determine } \\
\text { how timing of introduction of individual } \\
\text { food groups was analyzed; did not } \\
\text { report outcomes for length or BMl; Did } \\
\text { not account for high loss to follow-up } \\
\text { ( 52\%); Did not adjust for potential }\end{array}$ \\
\hline
\end{tabular}




\begin{tabular}{|c|c|c|}
\hline $\begin{array}{l}\text { Background Diet: } \\
\text { EBF duration: } 2.6 \mathrm{mo}, \mathrm{BF} \\
\text { duration: } 8.6 \mathrm{mo}\end{array}$ & $\begin{array}{l}\text { Weight: Measured by study personnel, to nearest } 0.1 \mathrm{~kg} \\
\text { Confounders accounted for: } \\
\text { Sex: } X \\
\text { Feeding practices: X (BF, EBF duration) } \\
\text { Birth size: } X \\
\text { Gestational age: } X \\
\text { Other: Gestational weight gain, maternal pre-pregnancy BMI }\end{array}$ & $\begin{array}{l}\text { key confounders (education, SES, } \\
\text { maternal age, race/ethnicity) }\end{array}$ \\
\hline $\begin{array}{l}\text { Moss, } 2014 \\
\text { Prospective cohort study; U.S. } \\
\text { Sample Size: } \\
\text { Baseline } N=9550 \\
\text { Analytic } N=7200 \text { at } 2 y, 6950 \\
\text { at } 4 y \\
\text { Attrition = } 25 \% \text { at } 2 y, 27 \% \text { at } 4 y \\
\text { Power Analysis and Sufficient } \\
\text { Sample Size: NR } \\
\text { Sex: } \\
\text { NR } \\
\frac{\text { Race/Ethnicity: }}{\text { NR }} \\
\text { Background Diet: } \\
\text { Ever BF: } 70 \% \\
\text { Age CFB introduced: } 24 \% \\
<4 \mathrm{mo}, 50 \% 4-5 \mathrm{mo}, 26 \%>6 \mathrm{mo}\end{array}$ & $\begin{array}{l}\text { Intervention/Exposure: } \\
\text { Age of CFB introduction: }<4,4-5, \geq 6 \mathrm{mo} \\
\text { Age: } 9 \text { mo } \\
\text { Assessment Methods: } \\
\text { Maternal interview } \\
\text { Outcomes: } \\
\text { Weight status } \\
\text { Age: } 2 y, 4 y \\
\text { Assessment Methods: } \\
\text { Weight status: BMl percentile calculated using measured } \\
\text { height/weight; classified as healthy weight }\left(<85^{\text {th }}\right) \text {, overweight }(85- \\
\left.94^{\text {th }}\right) \text {, or obese }\left(\geq 95^{\text {th }}\right) \\
\text { Confounders accounted for: } \\
\text { Education: } X \\
\text { SES: } X \\
\text { Sex: } X \\
\text { Maternal age: } X \\
\text { Race/ethnicity: } X \\
\text { Feeding practices: } X \\
\text { Birth size: } X \text { (Birth weight) }\end{array}$ & $\begin{array}{l}\text { Limitations: } \\
\text { Cannot determine whether groups } \\
\text { were similar at baseline on key } \\
\text { characteristics; cannot determine } \\
\text { whether outcome assessors were } \\
\text { blinded; Did not account for high loss } \\
\text { to follow-up ( }>20 \% \text { ); Did not adjust for } \\
\text { potential key confounders (gestational } \\
\text { age) }\end{array}$ \\
\hline $\begin{array}{l}\text { Neutzling, } 2009 \\
\text { Prospective Cohort Study; } \\
\text { Brazil } \\
\text { Sample Size: } \\
\text { Baseline N: } 1460 \\
\text { Analytic N: } 1204 \\
\text { Attrition }=18 \%\end{array}$ & $\begin{array}{l}\text { Intervention/Exposure: } \\
\text { Age of CFB introduction: }<4,>4 \mathrm{mo} \\
\text { Age: } 6,12 \mathrm{mo} \\
\text { Assessment Methods: } \\
\text { Maternal interview } \\
\text { Outcomes: } \\
\text { Weight status }\end{array}$ & $\begin{array}{l}\text { Limitations: } \\
\text { Cannot determine whether outcome } \\
\text { assessors were blinded; Did not adjust } \\
\text { for potential key confounders (maternal } \\
\text { age, gestational age) }\end{array}$ \\
\hline
\end{tabular}




\begin{tabular}{|c|c|c|}
\hline $\begin{array}{l}\text { Power Analysis and Sufficient } \\
\text { Sample Size: Yes, } \mathrm{N}=1,204 \text { (to } \\
\text { detect statistically significant } \\
\text { relative risks of } 0.7 \text { ) } \\
\frac{\text { Sex: }}{50 \% \text { Female }} \\
\text { Race/Ethnicity: } \\
71 \% \text { White, } 29 \% \text { Black or } \\
\text { mixed skin color } \\
\text { Background Diet: } \\
\text { BF duration: } 18 \%>12 \mathrm{mo} \text {; EBF: } \\
15 \%>4 \text { mo; Ever BF: } 96 \% \\
\text { Age of CFB introduction: } 49 \% \\
<4 \text { mo }\end{array}$ & $\begin{array}{l}\text { Age: } 11 \mathrm{y} \\
\text { Assessment Methods: } \\
\text { Weight status: risk of overweight (>85th percentile for BMI), and } \\
\text { obesity (>85th percentile for BMI, >90th percentile for skinfolds); BMI } \\
\text { percentile calculated using measured weight, height; } \\
\text { triceps/subscapular skinfolds measured with calipers } \\
\text { Confounders accounted for: } \\
\text { Education: } X \\
\text { SES: } X \\
\text { Sex: } X \\
\text { Race/ethnicity: X } \\
\text { Feeding practices: } X \\
\text { Birth size: } X \\
\text { Other: Smoking, maternal pregestational BMI }\end{array}$ & \\
\hline $\begin{array}{l}\text { Pan, } 2016 \\
\text { Prospective Cohort Study; U.S } \\
\text { Sample Size: } \\
\text { Baseline N: } 1542 \\
\text { Analytic N: } 1189 \\
\text { Attrition: } 22.9 \% \\
\text { Power Analysis and Sufficient } \\
\text { Sample Size: NR } \\
\text { Sex: } \\
50 \% \text { Female } \\
\text { Race/Ethnicity: } \\
88 \% \text { White non-Hispanic, } 3.4 \% \\
\text { Black non-Hispanic, } 4.8 \% \\
\text { Hispanic, } 3.9 \% \text { Other non- } \\
\text { Hispanic } \\
\text { Background Diet: } \\
\text { BF duration: } 48.6 \%<6 \text { mo ; } \\
51.4 \% \geq 6 \text { mo }\end{array}$ & $\begin{array}{l}\text { Intervention/Exposure: } \\
\text { Age of CFB introduction ( }<4 \mathrm{mo}, \mathrm{Y} / \mathrm{N}) \text {; Age of SSB (juice drinks, soft } \\
\text { drinks, soda, sweet tea, Kool-Aid) introduction: never, }<6 \mathrm{mo}, \geq 6 \mathrm{mo} \\
\text { Age: } 10-12 \mathrm{mo} \\
\text { Assessment Methods: } \\
\text { Caregiver questionnaire } \\
\text { Outcomes: } \\
\text { Weight status } \\
\text { Age: } 6 y \\
\text { Assessment Methods: } \\
\text { Obesity: based on BMl-for-age } \geq 95 \text { th \%tile, calculated from } \\
\text { maternal-report of weight and height taken with scale and tape } \\
\text { Confounders accounted for: } \\
\text { Education: } X \\
\text { SES: } X \\
\text { Sex: } X \\
\text { Maternal age: } X \\
\text { Race/ethnicity: } X \\
\text { Feeding practices: } X \\
\text { Birth size: } X\end{array}$ & $\begin{array}{l}\text { Limitations: } \\
\text { Cannot determine reliability/validity of } \\
\text { outcome assessment and outcome } \\
\text { assessors were not blinded due to } \\
\text { both feeding and outcome data self- } \\
\text { reported by mothers; Did not assess } \\
\text { the impact of high loss to follow-up } \\
\text { ( 22.9\%); Did not adjust analyses for } \\
\text { potential confounders (gestational } \\
\text { age) } \\
\text { Limited generalizability due to sample } \\
\text { not being representative (Data not } \\
\text { derived from a nationally } \\
\text { representative sample, } \\
\text { underrepresented minority groups; } \\
\text { sample biased toward mothers with } \\
\text { more education and higher SES) }\end{array}$ \\
\hline
\end{tabular}




\begin{tabular}{|c|c|c|}
\hline & $\begin{array}{l}\text { Gestational age: } \\
\text { Other: age at CFB introduction; pregnancy weight status }\end{array}$ & \\
\hline $\begin{array}{l}\text { Piwoz, } 1996 \\
\text { Prospective Cohort Study; Peru } \\
\text { Sample Size: } \\
\text { Baseline N: } 156 \\
\text { Analytic N: } 140 \\
\text { Attrition: } 11 \% \\
\text { Power Analysis and Sufficient } \\
\text { Sample Size: NR } \\
\text { Sex: } \\
\text { NR } \\
\text { Race/Ethnicity: } \\
\text { NR } \\
\text { Background Diet: } \\
\text { EBF to } 4 \mathrm{mo:} 21 \%, \text { BF/FF at } \\
4 \text { mo: } 13 \% \\
\text { Age of introduction of CFB: } \\
61 \% \text { by } 4 \text { mo }\end{array}$ & $\begin{array}{l}\text { Intervention/Exposure: } \\
\text { Age of CFB introduction: }<4(\mathrm{BF} / \mathrm{FF}+\mathrm{CFB}),>4 \mathrm{mo}(\mathrm{BF} / \mathrm{FF}) \\
\text { Age: } 4 \mathrm{mo} \\
\text { Assessment Methods: } \\
\text { Caregiver questionnaire } \\
\text { Outcomes: } \\
\text { Weight } \\
\text { Age: birth-3mo; }>3 \mathrm{mo} \\
\text { Assessment Methods: } \\
\text { Weight: Measured by study personnel in duplicate, to nearest } 100 \mathrm{~g} \\
\text { Confounders accounted for: } \\
\text { Birth size: } X \text { (All with birth weight }>2.5 \mathrm{~kg})\end{array}$ & $\begin{array}{l}\text { Limitations: } \\
\text { Groups differed at baseline on key } \\
\text { characteristics that were not adjusted } \\
\text { for in analyses (birthweight, age of } \\
\text { formula and CFB introduction, calorie } \\
\text { intake, diarrhea and fever prevalence); } \\
\text { Cannot determine whether outcome } \\
\text { assessors were blinded; Did not adjust } \\
\text { for key confounders (sex, gestational } \\
\text { age, race/ethnicity); Data and } \\
\text { statistical comparisons reported in } \\
\text { figures is not described (mean values, } \\
\text { P-values, etc.) } \\
\text { Limited generalizability of population } \\
\text { due to time of data collection; Cannot } \\
\text { rule out reverse causality (BF/FF } \\
\text { practices during age of outcome) }\end{array}$ \\
\hline $\begin{array}{l}\text { Quandt, } 1984 \\
\text { Prospective Cohort Study; U.S. } \\
\text { Sample Size: } \\
\text { Baseline N: } 91 \\
\text { Analytic N: } 45 \\
\text { Attrition: } 50 \% \\
\text { Power Analysis and Sufficient } \\
\text { Sample Size: NR } \\
\text { Sex: } \\
47 \% \text { Female } \\
\text { Race/Ethnicity: } \\
100 \% \text { White } \\
\text { Background Diet: } \\
\text { EBF at baseline: } 100 \%\end{array}$ & $\begin{array}{l}\text { Intervention/Exposure: } \\
\text { Age of CFB introduction: }<4,>4 \mathrm{mo} \\
\text { Age: } 2 \text { wk to } 6 \text { mo } \\
\text { Assessment Methods: } \\
\text { Food diaries, collected in real-time } \\
\text { Outcomes: } \\
\text { Body composition; Weight; Length } \\
\text { Age: } 1,2,3,4,5,6 \text { mo } \\
\text { Assessment Methods: } \\
\text { Body composition: WLZ calculated from measures } \\
\text { Weight and Length (recumbent): Measured by study personnel using } \\
\text { standard anthropometric techniques } \\
\text { Confounders accounted for: } \\
\text { None }\end{array}$ & $\begin{array}{l}\text { Limitations: } \\
\text { Cannot determine whether groups } \\
\text { were similar at baseline on key } \\
\text { characteristics; Cannot determine } \\
\text { whether outcome assessors were } \\
\text { blinded; Cannot determine the } \\
\text { validity/reliability of outcome } \\
\text { assessment (i.e., single vs multiple } \\
\text { growth measures); Did not account for } \\
\text { high loss to follow-up (50\%); Did not } \\
\text { adjust for potential key confounders } \\
\text { (education, SES, sex, maternal age, } \\
\text { race/ethnicity, feeding practices, birth } \\
\text { size, gestational age) }\end{array}$ \\
\hline
\end{tabular}




\begin{tabular}{|c|c|c|}
\hline $\begin{array}{l}\text { Age of CFB introduction: } \sim 120 \mathrm{~d} \\
(36-178 \mathrm{~d})\end{array}$ & & $\begin{array}{l}\text { Small sample size; Cannot rule out } \\
\text { reverse-causality (tming of CFB during } \\
\text { outcome assessment) }\end{array}$ \\
\hline $\begin{array}{l}\text { Reilly, } 2005 \\
\text { Prospective Cohort Study; } \\
\text { United Kingdom } \\
\text { Sample Size: } \\
\text { Baseline N: } 13971 \\
\text { Analytic N: } 5493 \\
\text { Attrition: } 61 \% \\
\text { Power Analysis and Sufficient } \\
\text { Sample Size: NR } \\
\text { Sex: } \\
\text { 49\% Female } \\
\text { Race/Ethnicity: } \\
86 \% \text { White } \\
\text { Background Diet: } \\
\text { EBF at 2mo: 28\%; Never BF: } \\
\text { 15\% }\end{array}$ & $\begin{array}{l}\text { Intervention/Exposure: } \\
\text { Age of CFB introduction: }<1,1-2,2-3,3-4,4-6,>6 \text { mo } \\
\text { Age: } 6 \text { mo } \\
\text { Assessment Methods: } \\
\text { Parent questionnaire } \\
\text { Outcomes: } \\
\text { Weight status } \\
\text { Age: } 7 y \\
\text { Assessment Methods: } \\
\text { Weight status: risk of obesity (BMI } \geq 95 \text { th \%tile); BMl calculated using } \\
\text { measured weight, height } \\
\text { Confounders accounted for: } \\
\text { Education: } X \\
\text { Sex: } X \\
\text { Maternal age: } X \\
\text { Race/ethnicity: } X \\
\text { Feeding practices: } X \\
\text { Birth size: } X \\
\text { Gestational age: } X \\
\text { Other: Energy intake at } 3 y, \text { parity, maternal smoking, season of birth, } \\
\text { \# of fetuses, parental obesity, siblings, time spent watching tv and in } \\
\text { the }\end{array}$ & $\begin{array}{l}\text { Limitations: } \\
\text { Cannot determine whether groups } \\
\text { were similar at baseline on key } \\
\text { characteristics; Cannot determine } \\
\text { whether outcome assessors were } \\
\text { blinded; Cannot determine the } \\
\text { validity/reliability of outcome } \\
\text { assessment (i.e., single vs multiple } \\
\text { growth measures); Did not account for } \\
\text { high loss to follow-up ( } 61 \% \text { ); Did not } \\
\text { adjust for potential key confounders } \\
\text { (SES) }\end{array}$ \\
\hline $\begin{array}{l}\text { Robinson, } 2009 \\
\text { Prospective Cohort Study; U.K. } \\
\text { Sample Size: } \\
\text { Baseline N: } 1195 \\
\text { Analytic N: } 536 \\
\text { Attrition: } 55 \% \\
\text { Power Analysis and Sufficient } \\
\text { Sample Size: NR }\end{array}$ & $\begin{array}{l}\text { Intervention/Exposure: } \\
\text { Age of CFB introduction: Not defined } \\
\text { Age: } 6 \mathrm{mo}, 12 \mathrm{mo} \\
\text { Assessment Methods: } \\
\text { FFQ; maternal interview } \\
\text { Outcomes: } \\
\text { Body composition } \\
\text { Age: } 4 y\end{array}$ & $\begin{array}{l}\text { Limitations: } \\
\text { Cannot determine whether groups } \\
\text { were similar at baseline on key } \\
\text { characteristics; Cannot determine } \\
\text { whether outcome assessors were } \\
\text { blinded; Cannot determine the } \\
\text { validity/reliability of some outcome } \\
\text { measures (i.e., single vs multiple } \\
\text { height, weight measures); Did not }\end{array}$ \\
\hline
\end{tabular}




\begin{tabular}{|c|c|c|}
\hline $\begin{array}{l}\text { Sex: } \\
47 \% \text { Female } \\
\text { Race/Ethnicity: } \\
\text { NR } \\
\text { Background Diet: } \\
\text { Never BF: } 12.5 \% ; \text { BF for } 6 \mathrm{mo:} \\
20 \% \\
\text { Age of introduction to CFB: } \\
76 \% \text { by } 4 \mathrm{mo}\end{array}$ & $\begin{array}{l}\text { Assessment Methods: } \\
\text { Body composition: } \\
\text { - BMI: Calculated using measured height, weight } \\
\text { - Lean mass: Whole-body DXA measurement; Lean mass index: } \\
\text { lean mass divided by height }{ }^{2} \text {; } \\
\text { - Fat mass (FM): Whole-body DXA measurement; Fat mass index } \\
\text { (FMI): FM divided by height }{ }^{2} \\
\text { Confounders accounted for: } \\
\text { SES: } X \\
\text { Maternal age: X } \\
\text { Feeding practices: } X \\
\text { Birth size: } X \\
\text { Other: Maternal BMI, maternal height, smoking in late pregnancy }\end{array}$ & $\begin{array}{l}\text { define age of introduction of CFB } \\
\text { variable; Did not account for high loss } \\
\text { to follow-up ( } 55 \% \text { ); Did not adjust for } \\
\text { potential key confounders (education, } \\
\text { sex, race/ethnicity, gestational age) }\end{array}$ \\
\hline $\begin{array}{l}\text { Rossiter, } 2013 \\
\text { Prospective Cohort Study; } \\
\text { Canada } \\
\text { Sample Size: } \\
\text { Baseline N: } 546 \\
\text { Analytic N: } 377 \\
\text { Attrition: } 31 \% \\
\text { Power Analysis and Sufficient } \\
\text { Sample Size: NR } \\
\text { Sex: } \\
43 \% \text { Female } \\
\text { Race/Ethnicity: } \\
\text { NR } \\
\text { Background Diet: } \\
\text { BF initiated: } 75 \% ; \text { BF at 3mo: } \\
44 \% \\
\text { Age of CFB introduction: } 50 \% \\
<4 m o, 10 \%<2 \text { mo }\end{array}$ & $\begin{array}{l}\text { Intervention/Exposure: } \\
\text { Age of CFB introduction: }<4,>4 \mathrm{mo} \\
\text { Age: } 3,18,33 \text {, and } 48 \mathrm{mo} \\
\text { Assessment Methods: } \\
\text { Maternal interview } \\
\text { Outcomes: } \\
\text { Weight status } \\
\text { Age: } 4 y \\
\text { Assessment Methods: } \\
\text { Weight status: prevalance of overweight/obesity vs. normal/under } \\
\text { weight with overweight classified as BMl } \geq 85 \text { th percentile, and } \\
\text { obesity as BMl } \geq 95 \text { th percentile; BMl calculated using measured } \\
\text { (duplicate) weight, height } \\
\text { Confounders accounted for: } \\
\text { Education: } X \\
\text { SES: } X \\
\text { Sex: } X \\
\text { Maternal age: } X \\
\text { Feeding practices: } X \\
\text { Birth size: } X \\
\text { Gestational age: }\end{array}$ & $\begin{array}{l}\text { Limitations: } \\
\text { Cannot determine whether groups } \\
\text { were similar at baseline on key } \\
\text { characteristics; Cannot determine } \\
\text { whether outcome assessors were } \\
\text { blinded; Did not account for high loss } \\
\text { to follow-up ( } 31 \%) \text {; Did not adjust for } \\
\text { potential key confounders } \\
\text { (race/ethnicity, gestational age) }\end{array}$ \\
\hline
\end{tabular}




\begin{tabular}{|c|c|c|}
\hline & $\begin{array}{l}\text { Other: Maternal birthplace, smoking, siblings, marital status, number } \\
\text { of people in home, parental classes, maternal weight }\end{array}$ & \\
\hline $\begin{array}{l}\text { Salmenpera, } 1985 \\
\text { Prospective Cohort Study; } \\
\text { Finland } \\
\text { Sample Size: } \\
\text { Baseline N: } 198 \\
\text { Analytic N: at 6-9mo, N: } 113 \\
\text { (FF+CFB N: 32; EBF } \\
\text { N:36; BF+CFB N:45) } \\
\text { Analytic N: at 9-12mo, N: } 84 \\
\text { (FF+CFB N: 32; EBF } \\
\text { N:7; BF+CFB N:45) } \\
\text { Attrition: at 6-9mo, 42\%; at 9- } \\
\text { 12mo, 58\% } \\
\text { Power Analysis and Sufficient } \\
\text { Sample Size: NR } \\
\text { Sex: } \\
53 \% \text { Female } \\
\text { Race/Ethnicity: } \\
\text { NR } \\
\text { Background Diet: } \\
\text { BF at 6mo: 59\% } \\
\text { Age of CFB introduction: 42\% } \\
\text { between 4-6mo }\end{array}$ & $\begin{array}{l}\text { Intervention/Exposure: } \\
\text { Age of CFB introduction: } \mathrm{BF}+\mathrm{CFB}(6-9 \mathrm{mo} \text {, or } 9-12 \mathrm{mo}, \mathrm{N}: 45) \text { vs. EBF } \\
(6-9 \mathrm{mo}, \mathrm{N}: 36 \text { or } 9-12 \mathrm{mo}, \mathrm{N}: 7) \\
{ }^{*} \text { Article "comparison group" of } F F+C F B<3.5 \mathrm{mo} \text { of age is not } \\
\text { included here } \\
\text { Age: NR } \\
\text { Assessment Methods: } \\
\text { NR } \\
\text { Outcomes: } \\
\text { Weight; Length } \\
\text { Age: } 6-12 \text { mo } \\
\text { Assessment Methods: } \\
\text { Weight: velocity from weight measured by study personnel to } \\
\text { nearest } 5 \mathrm{~g} \\
\text { Length: velocity from recumbent length measured with length board } \\
\text { by study personnel to nearest } 1 \mathrm{~mm} \\
\text { Confounders accounted for: } \\
\text { Gestational age: } X\end{array}$ & $\begin{array}{l}\text { Limitations: } \\
\text { Cannot determine whether groups } \\
\text { were similar at baseline on key } \\
\text { characteristics; Cannot determine } \\
\text { whether outcome assessors were } \\
\text { blinded; Cannot determine the } \\
\text { validity/reliability of outcome } \\
\text { asssessment (i.e., single vs multiple } \\
\text { growth measures); limited information } \\
\text { provided regarding infant feeding } \\
\text { measures; Did not account for high } \\
\text { loss to follow-up (>40\%); Did not adjust } \\
\text { for key confounders (education, SES, } \\
\text { sex, maternal age, race/ethnicity, } \\
\text { feeding practices, birth size) } \\
\text { Data and statistical comparisons } \\
\text { reported in tables/figures for other } \\
\text { relevant outcomes and sub-groups } \\
\text { were not described (e.g., head } \\
\text { circumference and skinfold } \\
\text { comparisons not shown for BF+CFB } \\
\text { vs. EBF) } \\
\text { Article notes potential for reverse } \\
\text { causality (faster growing infants had } \\
\text { earlier timing of CFB/greater infant } \\
\text { demand) }\end{array}$ \\
\hline $\begin{array}{l}\text { Santorelli, } 2014 \\
\text { Prospective Cohort Study; } \\
\text { United Kingdom } \\
\text { Sample Size: } \\
\text { Baseline N: } 1735 \\
\text { Analytic N: } 1327 \\
\text { Attrition: } 24 \%\end{array}$ & $\begin{array}{l}\text { Intervention/Exposure: } \\
\text { Age of CFB introduction: }<17,>17 \mathrm{wk} \\
\text { CFB: sweetened foods a/o drinks } \\
\text { Age: } 6,12 \mathrm{mo} \\
\text { Assessment Methods: } \\
\text { Maternal questionnaire }\end{array}$ & $\begin{array}{l}\text { Limitations: } \\
\text { Cannot determine whether groups } \\
\text { were similar at baseline on key } \\
\text { characteristics; Cannot determine } \\
\text { whether outcome assessors were } \\
\text { blinded; Cannot determine the } \\
\text { validity/reliability of outcome measures } \\
\text { (i.e., single vs multiple growth }\end{array}$ \\
\hline
\end{tabular}




\begin{tabular}{|c|c|c|}
\hline $\begin{array}{l}\text { Power Analysis and Sufficient } \\
\text { Sample Size: NR } \\
\text { Sex: } \\
\text { NR } \\
\text { Race/Ethnicity: } \\
38 \% \text { White, } 49 \% \text { Pakistani, } 7 \% \\
\text { South Asian, } 6 \% \text { Other } \\
\text { Background Diet: } \\
\text { BF after CFB introduction: } 32 \% \\
\text { Age of CFB introduction: } 10 \% \\
<17 \text { wk }\end{array}$ & $\begin{array}{l}\text { Outcomes: } \\
\text { Body composition } \\
\text { Age: } 3 y \\
\text { Assessment Methods: } \\
\text { Body composition: BMIZ: calculated using measured height, weight } \\
\text { and converted into age- and sex-adjusted z-scores based on WHO, } \\
2006 \text { growth standards } \\
\text { Confounders accounted for: } \\
\text { Education: } X \\
\text { Sex: } X \\
\text { Maternal age: } X \\
\text { Race/ethnicity: } X \\
\text { Feeding practices: } X \\
\text { Birth size: } X \\
\text { Gestational age: X } \\
\text { Other: Marital status, smoking during pregnancy, maternal BMI, } \\
\text { delivery mode }\end{array}$ & $\begin{array}{l}\text { measures); Did not account for high } \\
\text { loss to follow-up ( } 24 \% \text { ); Did not adjust } \\
\text { for potential key confounders (SES) }\end{array}$ \\
\hline $\begin{array}{l}\text { Schack-Nielsen, } 2010 \\
\text { Prospective Cohort Study; } \\
\text { Denmark } \\
\text { Sample Size: } \\
\text { Baseline N: } 8129 \\
\text { Analytic N: } 5068 \\
\text { Attrition: } 39 \% \\
\text { Power Analysis/Sample Size } \\
\text { Calculation: NR } \\
\text { Sex: } \\
\text { NR } \\
\text { Race/Ethnicity: } \\
\text { NR } \\
\text { Background Diet: } \\
\text { BF duration: 3.5mo; BF <2wk: } \\
\text { Ag\% of CFB introduction: } 3.8 m o\end{array}$ & $\begin{array}{l}\text { Intervention/Exposure: } \\
\text { Age of introduction to CFB: Continuous, mo; Categorical, } \geq 4 \text { vs } \\
<4 \text { mo } \\
\text { CFB: spoon-feeding, vegetables, eggs, meat, firm food } \\
\text { Age: } 1 y \\
\text { Assessment Methods: } \\
\text { Maternal questionnaire } \\
\text { Outcomes: } \\
\text { Weight status; Body composition; WC } \\
\text { Age: } 1-42 y \\
\text { Assessment Methods: } \\
\text { Weight status: risk of overweight based on BMI } \geq 25 \text { using self- } \\
\text { reported height and weight at } 42 y \\
\text { Body composition: BMI calculated using height, weight measured by } \\
\text { study personnel at } 1,3 \text {, and } 6 y ; \text { school records at } 7-14 y ; \text { self- } \\
\text { reported at } 2-34 y, 42 y \\
\text { WC: Self-reported at } 42 y \text { via mailed questionnaire and tape measure } \\
\text { Confounders accounted for: }\end{array}$ & $\begin{array}{l}\text { Limitations: } \\
\text { Cannot determine whether groups } \\
\text { were similar at baseline on key } \\
\text { characteristics; Outcome assessors } \\
\text { were not blinded at 20-34y, 42y; Did } \\
\text { not use valid/reliable measures to } \\
\text { assess outcomes (weight was self- } \\
\text { reported at 20-34y and 42y); Did not } \\
\text { account for high loss to follow-up } \\
\text { (39\%); Did not adjust for potential key } \\
\text { confounders (race/ethnicity); Not all } \\
\text { variables were analyzed in relation to } \\
\text { all outcomes, and data from figures not } \\
\text { adequately described } \\
\text { Limited generalizability (CFB practices } \\
\text { in 1959-1960) }\end{array}$ \\
\hline
\end{tabular}




\begin{tabular}{|c|c|c|}
\hline & $\begin{array}{l}\text { Education: } X \\
\text { SES: } X \\
\text { Sex: } X \\
\text { Maternal age: } X \\
\text { Race/ethnicity: } \\
\text { Feeding practices: } X \\
\text { Birth size: } X \text { (Birth weight) } \\
\text { Gestational age: } X \\
\text { Other: Prepregnancy BMI, gestational weight gain, smoking during } \\
\text { pregnancy, marital status, weight at } 1 \mathrm{y}\end{array}$ & \\
\hline $\begin{array}{l}\text { Seach, } 2010 \\
\text { Prospective Cohort Study; } \\
\text { Australia } \\
\text { Sample Size: } \\
\text { Baseline N: } 620 \\
\text { Analytic N: } 307 \\
\text { Attrition: } 50 \% \\
\text { Power Analysis and Sufficient } \\
\text { Sample Size: NR } \\
\text { Sex: } \\
\text { NR } \\
\text { Race/Ethnicity: } \\
99 \% \text { White } \\
\text { Background Diet: } \\
\text { Ever BF: } 94 \% \text {; EBF duration: } \\
\text { 15wk; BF duration: 48wk } \\
\text { Age of CFB introduction: } 20 w k \\
\text { (6\% >6mo) }\end{array}$ & $\begin{array}{l}\text { Intervention/Exposure: } \\
\text { Age of CFB introduction: Continuous, wk } \\
\text { Age: } 0-64 w k \text { (weekly), 78wk, 2y } \\
\text { Assessment Methods: } \\
\text { Maternal interview } \\
\text { Outcomes: } \\
\text { Weight status } \\
\text { Age: } 10 y \\
\text { Assessment Methods: } \\
\text { Weight status: BMl calculated using measured height, weight } \\
\text { (methods NR); classified into age- and sex-standardized BMl } \\
\text { categories (normal weight, overweight and obese); overweight and } \\
\text { obese were combined to create a binary outcome variable of an } \\
\text { 'above healthy' BMI vs. 'healthy' } \\
\text { Confounders accounted for: } \\
\text { Education: X } \\
\text { SES: } X \\
\text { Race/ethnicity: } X \\
\text { Feeding practices: } X \\
\text { Birth size: } X \\
\text { Other: Parental smoking, childcare }\end{array}$ & $\begin{array}{l}\text { Limitations: } \\
\text { Cannot determine whether groups } \\
\text { were similar at baseline on key } \\
\text { characteristics; Cannot determine } \\
\text { whether outcome assessors were } \\
\text { blinded; Cannot determine the } \\
\text { validity/reliability of outcome measures } \\
\text { (i.e., single vs multiple growth } \\
\text { measures); Did not account for high } \\
\text { loss to follow-up (50\%); Did not adjust } \\
\text { for potential key confounders (sex, } \\
\text { maternal age, gestational age) }\end{array}$ \\
\hline $\begin{array}{l}\text { van Rossem, } 2013 \\
\text { Prospective Cohort Study; The } \\
\text { Netherlands }\end{array}$ & $\begin{array}{l}\text { Intervention/Exposure: } \\
\text { Age of CFB introduction: } 0-3,3-6,>6 \mathrm{mo} \\
\text { Age: } 12 \mathrm{mo}\end{array}$ & $\begin{array}{l}\text { Limitations: } \\
\text { Birth weight differed between timing of } \\
\text { CFB groups, and was not adjusted for } \\
\text { in analyses (all subjects were normal }\end{array}$ \\
\hline
\end{tabular}




\begin{tabular}{|c|c|c|}
\hline $\begin{array}{l}\text { Sample Size: } \\
\text { Baseline N: } 7295 \\
\text { Analytic N: } 3184 \\
\text { Attrition: } 56 \% \\
\text { Power Analysis and Sufficient } \\
\text { Sample Size: NR } \\
\text { Sex: } \\
\text { NR } \\
\text { Race/Ethnicity: } \\
68 \% \text { Dutch, } 12 \% \text { Other } \\
\text { Western, } 21 \% \text { Non-Western } \\
\text { Background Diet: } \\
\text { BF at } 2 \mathrm{mo}: 69 \% \text {, BF at } 6 \mathrm{mo:} \\
33 \% \\
\text { Age of CFB introduction: } 5 \% 0 \text { - } \\
3 \mathrm{mo}, 57 \% 3-6 \mathrm{mo}, 38 \%>6 \mathrm{mo}\end{array}$ & $\begin{array}{l}\text { Assessment Methods: } \\
\text { Maternal questionnaire } \\
\text { Outcomes: } \\
\text { Body composition } \\
\text { Age: } 12-45 \text { mo } \\
\text { Assessment Methods: } \\
\text { Body composition: } \\
\text { - WLZ/WHZ: change in weight-for-length/height (kg/cm gain in } \\
\text { length) z score according to Dutch reference; weight (SECA } \\
\text { scale), length/height (supine/stadiometer) were measured by study } \\
\text { personnel } \\
\text { Confounders accounted for: } \\
\text { Education: } X \\
\text { Race/ethnicity: } X \\
\text { Feeding practices: } X \\
\text { Gestational age: } X \text { (All >37wk) } \\
\text { Other: Smoking during pregnancy, maternal BMI, history of allergy or } \\
\text { hospital admission in the first year of life }\end{array}$ & $\begin{array}{l}\text { birth weight); Cannot determine if } \\
\text { outcome assessors were blinded; } \\
\text { Cannot determine the validity/reliability } \\
\text { of outcome measures (i.e., single vs } \\
\text { multiple measures); Did not adjust for } \\
\text { several potential key confounders } \\
\text { (SES, sex, maternal age, birth size); } \\
\text { Adequacy of statistical methods } \\
\text { insufficient (statistical comparisons } \\
\text { between different timing of CFB } \\
\text { introduction were not clearly made in } \\
\text { tables, figures, or text) } \\
\text { Infants introduced to CFB earlier had } \\
\text { higher WLZ before CFB introduction; } \\
\text { confidence intervals in tables indicate } \\
\text { significance but comparisons were not } \\
\text { clear }\end{array}$ \\
\hline $\begin{array}{l}\text { Victora, } 1998 \\
\text { Prospective Cohort Study; } \\
\text { Brazil } \\
\text { Sample Size: } \\
\text { Baseline N: } 655 \\
\text { Analytic N: } 627 \\
\text { Attrition: } 4 \% \\
\text { Power Analysis and Sufficient } \\
\text { Sample Size: NR } \\
\text { Sex: } \\
51 \% \text { Female } \\
\text { Race/Ethnicity: } \\
\text { NR } \\
\text { Background Diet: } \\
\text { BF: } 100 \%\end{array}$ & $\begin{array}{l}\text { Intervention/Exposure: } \\
\text { Age of CFB introduction: }<3,>3 \mathrm{mo} \\
\text { Age: } 1,3,6 \text { mo } \\
\text { Assessment Methods: } \\
\text { 24-hr recall } \\
\text { Outcomes: } \\
\text { Body composition; Weight; Length } \\
\text { Age: } 3-6 \text { mo } \\
\text { Assessment Methods: } \\
\text { Body composition: ponderal index derived from weight divided by } \\
\text { length } 3 \\
\text { Weight: Measured by study personnel, to nearest } 100 \mathrm{~g} \\
\text { Length: Measured by study personnel, recumbent } \\
\text { Confounders accounted for: } \\
\text { Education: } X\end{array}$ & $\begin{array}{l}\text { Limitations: } \\
\text { Cannot determine whether groups } \\
\text { were similar at baseline on key } \\
\text { characteristics; Cannot determine } \\
\text { whether outcome assessors were } \\
\text { blinded; Cannot determine the } \\
\text { validity/reliability of outcome } \\
\text { assessment (i.e., single vs multiple } \\
\text { measures); Did not adjust for potential } \\
\text { key confounders (sex, maternal age, } \\
\text { race/ethnicity, feeding practices, birth } \\
\text { size, gestational age) } \\
\text { Limited generalizability due to study } \\
\text { setting (lower HDI at the time of data } \\
\text { collection) }\end{array}$ \\
\hline
\end{tabular}




\begin{tabular}{|c|c|c|}
\hline & $\begin{array}{l}\text { SES: } X \\
\text { Other: Housing quality, crowding index, number of children }<5 y \text { in } \\
\text { house }\end{array}$ & \\
\hline $\begin{array}{l}\text { Villalpando, } 2000 \\
\text { Prospective Cohort Study; } \\
\text { Mexico } \\
\text { Sample Size: } \\
\text { Baseline N: } 216 \\
\text { Analytic N: } 170 \\
\text { Attrition: } 21 \% \\
\text { Power Analysis and Sufficient } \\
\text { Sample Size: NR } \\
\text { Sex: } \\
\text { NR } \\
\text { Race/Ethnicity: } \\
\text { NR } \\
\text { Background Diet: } \\
\text { BF at birth: } 90 \% ; \text { BF at } 6 \mathrm{mo:} \\
25 \% \\
\text { Age of CFB introduction: } 86 \% \\
\text { by } 4 \text { mo }\end{array}$ & $\begin{array}{l}\text { Intervention/Exposure: } \\
\text { Age of CFB introduction: } 15 \mathrm{~d} \text { intervals from } 0-6 \mathrm{mo} \text {, Continuous } \\
\text { Age: } 0-6 \mathrm{mo} \\
\text { Assessment Methods: } \\
\text { Maternal interview, every } 15 \mathrm{~d} \\
\text { Outcomes: } \\
\text { Weight; Length } \\
\text { Age: } 0-6 \mathrm{mo} \\
\text { Assessment Methods: } \\
\text { Weight: Measured by study personnel with an electronic balance } \\
\text { Length: Measured by study personnel using a length board } \\
\text { Confounders accounted for: } \\
\text { Feeding practices: } X \\
\text { Birth size: X (All with birth weight } 2500-4000 \mathrm{~g} \text {; Birth BMI) } \\
\text { Gestational age: X (All full term) } \\
\text { Other: Age, episodes of infection }\end{array}$ & $\begin{array}{l}\text { Limitations: } \\
\text { Cannot determine whether groups } \\
\text { were similar at baseline on key } \\
\text { characteristics; Cannot determine } \\
\text { whether outcome assessors were } \\
\text { blinded; Cannot determine the } \\
\text { validity/reliability of outcome } \\
\text { assessment (i.e., insufficient detail } \\
\text { regarding single vs multiple measures); } \\
\text { Did not account for high loss to follow- } \\
\text { up ( } 21 \% \text { ); Did not adjust for potential } \\
\text { key confounders (education, SES, sex, } \\
\text { maternal age, race/ethnicity) } \\
\text { Limited generalizability (sample from } \\
\text { slum population); Cannot rule out } \\
\text { reverse causaliy (timing of CFB during } \\
\text { outcome assessment) }\end{array}$ \\
\hline $\begin{array}{l}\text { Warrington, } 1988 \\
\text { Prospective Cohort Study; U.K. } \\
\text { Sample Size: } \\
\text { Baseline N: } 109 \\
\text { Analytic N: } 78 \\
\text { Attrition: } 28 \% \\
\text { Power Analysis and Sufficient } \\
\text { Sample Size: NR } \\
\text { Sex: } \\
\text { NR } \\
\text { Race/Ethnicity: } \\
50 \% \text { White, } 50 \% \text { Asian }\end{array}$ & $\begin{array}{l}\text { Intervention/Exposure: } \\
\text { Age of CFB introduction: Continuous, wk } \\
\text { Age: } 3,6,912 \text {, and } 24 \mathrm{mo} \\
\text { Assessment Methods: } \\
\text { Food records, 3d, weighed } \\
\text { Outcomes: } \\
\text { Weight } \\
\text { Age: } 1 \mathrm{y}, 2 \mathrm{y} \\
\text { Assessment Methods: } \\
\text { Weight: Measured by study personnel } \\
\text { Confounders accounted for: } \\
\text { Race/ethnicity: } X\end{array}$ & $\begin{array}{l}\text { Limitations: } \\
\text { Cannot determine whether groups } \\
\text { were similar at baseline on key } \\
\text { characteristics; Cannot determine if } \\
\text { adherence was similar across study } \\
\text { groups; Cannot determine whether } \\
\text { outcome assessors were blinded; } \\
\text { Cannot determine the validity/reliability } \\
\text { of outcome assessment (methods not } \\
\text { described); Did not account for high } \\
\text { loss to follow-up (28\%); Did not adjust } \\
\text { for potential key confounders } \\
\text { (Education, SES, sex, maternal age, } \\
\text { feeding practices, birth size, }\end{array}$ \\
\hline
\end{tabular}




\begin{tabular}{|c|c|c|}
\hline $\begin{array}{l}\text { Background Diet: } \\
\text { EBF for } 12 w k+: 5 \% ; \text { FF from } \\
\text { birth: } 40 \% \\
\text { Age of CFB introduction: } 46 \% \\
<12 w k\end{array}$ & & $\begin{array}{l}\text { gestational age); Adequacy of } \\
\text { statistical methods insufficient (did not } \\
\text { provide data table or statistics to } \\
\text { accompany results reported via figure } \\
\text { and text) }\end{array}$ \\
\hline $\begin{array}{l}\text { Wells, } 1998 \\
\text { Prospective Cohort Study; U.K. } \\
\text { Sample Size: } \\
\text { Baseline N: } 50 \\
\text { Analytic N: } 20 \\
\text { Attrition: } 60 \% \\
\text { Power Analysis and Sufficient } \\
\text { Sample Size: NR } \\
\text { Sex: } \\
\text { NR } \\
\text { Race/Ethnicity: } \\
\text { NR } \\
\text { Background Diet: } \\
\text { BF and FF }\end{array}$ & $\begin{array}{l}\text { Intervention/Exposure: } \\
\text { Age of CFB introduction: }<12,>12 w k \\
\text { Age: } 12 w k \\
\text { Assessment Methods: } \\
\text { Food records, } 1 \mathrm{~d} \text {, weighed } \\
\text { Outcomes: } \\
\text { Body composition (Adiposity); Weight; Height } \\
\text { Age: } 2-3.5 y \\
\text { Assessment Methods: } \\
\text { Body composition: } \\
\text { - FM, FFM: Calculated using total body water and weight } \\
\text { - Skinfold thickness (triceps, subscapular): Measured by study } \\
\text { personnel } \\
\text { Weight: Measured by study personnel } \\
\text { Height: Measured by study personnel } \\
\text { Confounders accounted for: } \\
\text { Feeding practices: } X \text { (energy intake, milk volume intake) } \\
\text { Gestational age: } X \text { (all full term) } \\
\text { Other: Child age, infant soothability score }\end{array}$ & $\begin{array}{l}\text { Limitations: } \\
\text { Cannot determine whether groups } \\
\text { were similar at baseline on key } \\
\text { characteristics; Cannot determine } \\
\text { whether outcome assessors were } \\
\text { blinded; Cannot determine the } \\
\text { validity/reliability of outcome } \\
\text { assessment (i.e., single vs multiple } \\
\text { measures); Length of follow-up ranged } \\
\text { from 2-3.5y; Did not account for high } \\
\text { loss to follow-up (60\%); Did not adjust } \\
\text { for potential key confounders } \\
\text { (education, SES, sex, maternal age, } \\
\text { birth size); Did not provide data table to } \\
\text { accompany all results reported in text }\end{array}$ \\
\hline $\begin{array}{l}\text { Wen, 2014b } \\
\text { Prospective Cohort Study; } \\
\text { Australia } \\
\text { Sample Size: } \\
\text { Baseline N: } 330 \\
\text { Analytic N: } 242 \\
\text { Attrition: } 27 \%\end{array}$ & $\begin{array}{l}\text { Intervention/Exposure: } \\
\text { Age of CFB introduction: }<2,3-5,>6 \mathrm{mo} \\
\text { Age: } 6 \mathrm{mo} \\
\text { Assessment Methods: } \\
\text { Maternal interview } \\
\text { Outcomes: } \\
\text { Weight status; Body composition } \\
\text { Age: } 2 \mathrm{y}\end{array}$ & $\begin{array}{l}\text { Limitations: } \\
\text { Cannot determine whether groups } \\
\text { were similar at baseline on key } \\
\text { characteristics; Cannot determine } \\
\text { whether outcome assessors were } \\
\text { blinded; Did not account for high loss } \\
\text { to follow-up (27\%); Did not adjust for }\end{array}$ \\
\hline
\end{tabular}




\begin{tabular}{|c|c|c|}
\hline $\begin{array}{l}\text { Power Analysis and Sufficient } \\
\text { Sample Size: NR } \\
\text { Sex: } \\
54 \% \text { Female } \\
\text { Race/Ethnicity: } \\
\text { NR } \\
\text { Background Diet: } \\
\text { Duration of BF: } 5.82 \mathrm{mo} \text {; Ever } \\
\text { BF: } 97 \% \\
\text { Age of CFB introduction: } 8 \% \\
<2 \mathrm{mo}, 28 \% 3-5 \mathrm{mo}, 64 \%>6 \mathrm{mo}\end{array}$ & $\begin{array}{l}\text { Assessment Methods: } \\
\text { Weight status: Overweight classified as BMl>25kg/m2, obese as } \\
\text { BMl>30kg/m2 } \\
\text { Body composition: BMI calculated using measured (duplicate) } \\
\text { weight, length } \\
\text { Confounders accounted for: } \\
\text { SES: } X \text { (Household income; employment status) } \\
\text { Sex: } X \\
\text { Education: } X \\
\text { Feeding practices: } X \\
\text { Birth size: } X \text { (birth weight) } \\
\text { Other: Marital status, tv time, mothers BMl and weight status, } \\
\text { mothers county of birth; age of starting tummy time; using bottle at } \\
\text { 1y; outdor activity time; food for reward; dietary intake at } 2 y ; \\
\text { maternal age }\end{array}$ & $\begin{array}{l}\text { potential key confounders } \\
\text { (race/ethnicity, gestational age) } \\
\text { Limited generalizability (sample was } \\
\text { "very disadvantaged") }\end{array}$ \\
\hline $\begin{array}{l}\text { WHO, } 2002 \\
\text { Prospective Cohort Study; } \\
\text { China, India, Guatemala, } \\
\text { Nigeria, Chile, Sweden, } \\
\text { Australia } \\
\text { Sample Size: } \\
\text { Baseline N: NR } \\
\text { Analytic N: } 1252 \\
\text { Attrition: CD } \\
\text { Power Analysis and Sufficient } \\
\text { Sample Size: NR } \\
\text { Sex: } \\
45 \% \text { Female } \\
\text { Race/Ethnicity: } \\
\text { NR } \\
\text { Background Diet: } \\
\text { BF: } 100 \%\end{array}$ & $\begin{array}{l}\text { Intervention/Exposure: } \\
\text { Age of CFB introduction: } 1-8,9-16,25-32 w k \text { vs } 17-24 w k \\
\text { Age: } 0-32 w k \\
\text { Assessment Methods: } \\
\text { Maternal interview } \\
\text { Outcomes: } \\
\text { Weight; Length } \\
\text { Age: } 8 w k \text { (midpoint of } 1-16 w k), 24 w k \text { (midpoint of 17-32wk) } \\
\text { Assessment Methods: } \\
\text { Weight: Measured by study personnel } \\
\text { Length: Measured by study personnel } \\
\text { Confounders accounted for: } \\
\text { Education: } X \\
\text { Sex: } X \\
\text { Maternal age: } X \\
\text { Feeding practices: } X \text { (BF duration, food given to infant before BF, } \\
\text { access of infant to breast, BF per day) } \\
\text { Birth size: } X \text { (all with birth weight }>2500 \mathrm{~g} \text { ) } \\
\text { Gestational age: } X\end{array}$ & $\begin{array}{l}\text { Limitations: } \\
\text { Cannot determine whether groups } \\
\text { were similar at baseline on key } \\
\text { characteristics; Cannot determine } \\
\text { whether outcome assessors were } \\
\text { blinded; Cannot determine loss to } \\
\text { follow-up, baseline sample size not } \\
\text { reported; Did not adjust for potential } \\
\text { key confounders (SES, race/ethnicity); } \\
\text { Statistical analyses were difficult to } \\
\text { interpret (i.e., in several cases, the } \\
\text { exposure of CFB introduction occurred } \\
\text { after the time point at which the } \\
\text { outcome was measured) } \\
\text { Results showed small differences in } \\
\text { growth based on timing of CFB } \\
\text { introduction that were statistically } \\
\text { significant but probably not biologically } \\
\text { important }\end{array}$ \\
\hline
\end{tabular}




\begin{tabular}{|c|c|c|}
\hline & Other: maternal surgery, number of previous births, maternal health & $\begin{array}{l}\text { Limited generalizability (exclusively } \\
\text { enrolled high SES) }\end{array}$ \\
\hline $\begin{array}{l}\text { Wolman, } 1984 \\
\text { Prospective Cohort Study; } \\
\text { United States } \\
\text { Sample Size: } \\
\text { Baseline N: } 262 \\
\text { Analytic N: } 164 \\
\text { Attrition: } 37 \% \\
\text { Power Analysis and Sufficient } \\
\text { Sample Size: NR } \\
\text { Sex: } \\
\text { NR } \\
\text { Race/Ethnicity: } \\
\text { NR } \\
\text { Background Diet: } \\
\text { BF 1-12 wk: } 16 \% ; \text { BF >13wk: } \\
5 \% \\
\text { Age of introduction to CFB: } \\
89 \%<3 \text { mo; } 65 \% \text { before } 1 \mathrm{mo}\end{array}$ & $\begin{array}{l}\text { Intervention/Exposure: } \\
\text { Age of CFB introduction: }<12,>13 w k \\
\text { Age: } 0-24 m o \\
\text { Assessment Methods: } \\
\text { Parent questionnaire } \\
\text { Outcomes: } \\
\text { Body composition (BMI); Weight; Height } \\
\text { Age: 4-6y } \\
\text { Assessment Methods: } \\
\text { Body composition: } \\
\text { - BMI calculated using height, weight taken from medical records } \\
\text { Weight: Taken from medical records } \\
\text { Height: Taken from medical records } \\
\text { Confounders accounted for: } \\
\text { None }\end{array}$ & $\begin{array}{l}\text { Limitations: } \\
\text { Cannot determine whether groups } \\
\text { were similar at baseline on key } \\
\text { characteristics; Cannot determine } \\
\text { whether outcome assessors were } \\
\text { blinded; Did not use valid/reliable } \\
\text { measures to assess outcomes (growth } \\
\text { measures taken from medical records); } \\
\text { Cannot determine whether length of } \\
\text { follow-up was similar across groups } \\
\text { (follow-up age ranged from 4-6y); Did } \\
\text { not account for high loss to follow-up } \\
\text { (37\%); Did not adjust for potential key } \\
\text { confounders (SES, sex, education, } \\
\text { maternal age, race/ethnicity, feeding } \\
\text { practices, birth size, gestational age) }\end{array}$ \\
\hline $\begin{array}{l}\text { Woo, } 2013 \\
\text { Prospective Cohort Study; } \\
\text { United States, Mexico, China } \\
\text { Sample Size: } \\
\text { Baseline N: } 365 \\
\text { Analytic N: } 285 \\
\text { Attrition: } 22 \% \\
\text { Power Analysis and Sufficient } \\
\text { Sample Size: NR } \\
\text { Sex: } \\
\text { NR } \\
\text { Race/Ethnicity: } \\
33 \% \text { US, 33\% Mexican, 33\% } \\
\text { Chinese }\end{array}$ & $\begin{array}{l}\text { Intervention/Exposure: } \\
\text { Age of CFB introduction: Continuous, mo } \\
\text { Age: } 0 \text {-1y } \\
\text { Assessment Methods: } \\
\text { 24-hr food frequency recall of } 21 \text { food items, weekly } \\
\text { Outcomes: } \\
\text { Body composition (BMI); Weight; Length } \\
\text { Age: } 1 y \\
\text { Assessment Methods: } \\
\text { Body composition: } \\
\text {-BMI calculated using measured height, weight } \\
\text { Weight: Measured by study personnel in duplicate } \\
\text { Length: Measured by study personnel in duplicate, supine }\end{array}$ & $\begin{array}{l}\text { Limitations: } \\
\text { Cannot determine whether groups } \\
\text { were similar at baseline on key } \\
\text { characteristics; Cannot determine } \\
\text { whether outcome assessors were } \\
\text { blinded; Did not account for high loss } \\
\text { to follow-up (22\%); Did not adjust for } \\
\text { key confounders (SES, race/ethnicity) }\end{array}$ \\
\hline
\end{tabular}




\begin{tabular}{|c|c|c|}
\hline $\begin{array}{l}\text { Background Diet: } \\
\text { EBF duration: 5wk in China, } \\
\text { 14wk in US, 7wk in Mexico; BF } \\
\text { duration: } 37 w k \text { in China, 50wk } \\
\text { in US, 52wk in Mexico } \\
\text { Age of CFB introduction: 18wk } \\
\text { in China, 23wk in US, 25wk in } \\
\text { Mexican }\end{array}$ & $\begin{array}{l}\text { Confounders accounted for: } \\
\text { Education: } X \\
\text { Sex: } X \\
\text { Maternal age: } X \\
\text { Feeding practices: } X \\
\text { Birth size: } X \\
\text { Gestational age: } X \text { (All >37wk) } \\
\text { Other: Prepregnancy BMI, gestational weight gain, gestational } \\
\text { diabetes, type of delivery, cohort site }\end{array}$ & \\
\hline $\begin{array}{l}\text { Worobey, } 2009 \\
\text { Prospective Cohort Study; } \\
\text { United States } \\
\text { Sample Size: } \\
\text { Baseline N: } 242 \\
\text { Analytic N: } 96 \\
\text { Attrition: } 60 \% \\
\text { Power Analysis and Sufficient } \\
\text { Sample Size: NR } \\
\text { Sex: } \\
51 \% \text { Female } \\
\text { Race/Ethnicity: } \\
24 \% \text { Black, } 76 \% \text { Hispanic } \\
\text { Background Diet: } \\
\text { FF: } 100 \% \\
\text { Age of CFB introduction: } \\
4.23 \text { mo }\end{array}$ & $\begin{array}{l}\text { Intervention/Exposure: } \\
\text { Age of CFB introduction: Continuous, mo } \\
\text { Age: } 3,6,12 \text { mo } \\
\text { Assessment Methods: } \\
\text { Food record } \\
\text { Outcomes: } \\
\text { Weight } \\
\text { Age: } 3-6 \text { mo } \\
\text { Assessment Methods: } \\
\text { Weight: Measured by study personnel trained in reliable } \\
\text { measurement } \\
\text { Confounders accounted for: } \\
\text { Education: } X \\
\text { Sex: } X \\
\text { Maternal age: } X \\
\text { Race/ethnicity: } X \\
\text { Feeding practices: } X \text { (\# feeds per day) } \\
\text { Birth size: } X \\
\text { Other: Maternal BMI, weight and length gain from 0-3mo, maternal } \\
\text { sensitivity to infant signals, country of origin, prepregnancy BMI, } \\
\text { gestational weight gain }\end{array}$ & $\begin{array}{l}\text { Limitations: } \\
\text { Cannot determine whether groups } \\
\text { were similar at baseline on key } \\
\text { characteristics; Did not account for } \\
\text { high loss to follow-up ( } 60 \%) \text {; Did not } \\
\text { adjust for potential key confounders } \\
\text { (SES, gestational age) }\end{array}$ \\
\hline $\begin{array}{l}\text { Worobey, } 2014 \\
\text { Prospective Cohort Study; } \\
\text { United States }\end{array}$ & $\begin{array}{l}\text { Intervention/Exposure: } \\
\text { Age of CFB introduction: Continuous, mo } \\
\text { Age: } 3,6,12 \mathrm{mo}\end{array}$ & $\begin{array}{l}\text { Limitations: } \\
\text { Cannot determine whether groups } \\
\text { were similar at baseline on key } \\
\text { characteristics; Cannot determine }\end{array}$ \\
\hline
\end{tabular}




\begin{tabular}{|c|c|c|}
\hline $\begin{array}{l}\text { Sample Size: } \\
\text { Baseline N: } 242 \\
\text { Analytic N: } 154 \\
\text { Attrition: } 36 \% \\
\text { Power Analysis and Sufficient } \\
\text { Sample Size: NR } \\
\text { Sex: } \\
\text { NR } \\
\text { Race/Ethnicity: } \\
24 \% \text { Black, } 54 \% \text { Mexican, } 25 \% \\
\text { other Latino } \\
\text { Background Diet: } \\
\text { FF: } 100 \%\end{array}$ & $\begin{array}{l}\text { Assessment Methods: } \\
\text { Food record } \\
\text { Outcomes: } \\
\text { Weight } \\
\text { Age: } 3-6 \mathrm{mo} \\
\text { Assessment Methods: } \\
\text { Weight: Measured by study personnel trained in reliable } \\
\text { measurement } \\
\text { Confounders accounted for: } \\
\text { Feeding practices: } X \text { (\# of feeds at } 3 \mathrm{mo}) \\
\text { Birth size: X } \\
\text { Other: Maternal BMI, \# of cries at } 3 \mathrm{mo}, \# \text { of cry-feeds at } 3 \mathrm{mo} \text {, infant } \\
\text { weight at } 3 \mathrm{mo}, \text { total sleep at } 3 \mathrm{mo}\end{array}$ & $\begin{array}{l}\text { whether outcome assessors were } \\
\text { blinded; Did not account for high loss } \\
\text { to follow-up ( } 36 \% \text { ); Did not adjust for } \\
\text { potential key confounders (education, } \\
\text { SES, sex, maternal age, race/ethnicity, } \\
\text { gestational age) }\end{array}$ \\
\hline $\begin{array}{l}\text { Wilson, } 1998 \\
\text { Prospective Cohort Study; U.K. } \\
\text { Sample Size: } \\
\text { Baseline N: } 674 \\
\text { Analytic N: } 545 \\
\text { Attrition: } 19 \% \\
\text { Sample Size Calculation: NR } \\
\text { Sex: } \\
54 \% \text { Female } \\
\text { Race/Ethnicity: } \\
\text { NR } \\
\text { Background Diet: } \\
\text { EBF at } 15 w k: 26 \%, \text { partial BF } \\
\text { at 15wk: } 37 \% \text {, formula fed at } \\
\text { 15wk: } 37 \%\end{array}$ & $\begin{array}{l}\text { Intervention/Exposure: } \\
\text { Age of CFB introduction: }<15,>15 w k \\
\text { Age: } 0-2 y \\
\text { Assessment Methods: } \\
\text { Parent questionnaire } \\
\text { Outcomes: } \\
\text { Weight, height, BMI, \% body fat } \\
\text { Age: } 7 y \\
\text { Assessment Methods: } \\
\text { Body composition: } \\
\text { - BMI calculated using measured height, weight } \\
\text {-\% body fat calculated based on bioelectrical impedance (and } \\
\text { skinfold thickness NR) } \\
\text { Weight: Measured by study personnel } \\
\text { Height: Measured by study personnel } \\
\text { Confounders accounted for: } \\
\text { SES: } X \\
\text { Sex: } X \\
\text { Feeding practices: } x \\
\text { Birth size: } X \text { (Birth weight) } \\
\text { Other: Height, maternal height, weight at first solids }\end{array}$ & $\begin{array}{l}\text { Limitations: } \\
\text { Cannot determine whether groups } \\
\text { were similar at baseline on key } \\
\text { characteristics; Cannot determine } \\
\text { whether outcome assessors were } \\
\text { blinded; Cannot determine the } \\
\text { validity/reliability of outcome } \\
\text { assessment (i.e., single vs multiple } \\
\text { measures); Did not adjust for potential } \\
\text { key confounders (education, } \\
\text { race/ethnicity, gestational age) } \\
\text { Article states skinfold thickness } \\
\text { measures were taken but data NR }\end{array}$ \\
\hline
\end{tabular}




\begin{tabular}{|c|c|c|}
\hline $\begin{array}{l}\text { Yeung, } 1981 \\
\text { Prospective Cohort Study; } \\
\text { Canada } \\
\text { Sample Size: } \\
\text { Baseline N: } 403 \\
\text { Analytic N: } 316 \\
\text { Attrition: } 22 \% \\
\text { Power Analysis and Sufficient } \\
\text { Sample Size: NR } \\
\text { Sex: } \\
\text { NR } \\
\text { Race/Ethnicity: } \\
\text { NR } \\
\text { Background Diet: } \\
\text { BF: } 56 \% \text { at } 1 \mathrm{mo}, 39 \% \text { at } 3 \mathrm{mo}, \\
26 \% \text { at } 5 \mathrm{mo}, 21 \% \text { at } 6 \mathrm{mo}\end{array}$ & $\begin{array}{l}\text { Intervention/Exposure: } \\
\text { Age of CFB introduction: }<2,>2 \mathrm{mo} \\
\text { Age: } 1,3,5,6,7,8,10,12,15,18 \mathrm{mo} \\
\text { Assessment Methods: } \\
\text { Food record, } 4 \mathrm{~d} \\
\text { Outcomes: } \\
\text { Body composition } \\
\text { Age: } 6 \text { mo } \\
\text { Assessment Methods: } \\
\text { Body composition: } \\
\text { - Skinfold thickness sum of triceps and subscapular skinfolds } \\
\text { measured by study personnel } \\
\text { Confounders accounted for: } \\
\text { Gestational age: } X \text { (All full term) }\end{array}$ & $\begin{array}{l}\text { Limitations: } \\
\text { Cannot determine whether groups } \\
\text { were similar at baseline on key } \\
\text { characteristics; Cannot determine } \\
\text { whether outcome assessors were } \\
\text { blinded; Cannot determine the } \\
\text { validity/reliability of outcome } \\
\text { assessment (i.e., single vs multiple } \\
\text { measures); Did not account for high } \\
\text { loss to follow-up (22\%); Did not adjust } \\
\text { for ptoential key confounders } \\
\text { (education, SES, sex, maternal age, } \\
\text { race/ethnicity, feeding practices, birth } \\
\text { size) }\end{array}$ \\
\hline $\begin{array}{l}\text { Zheng, } 2015 \\
\text { Prospective Cohort Study; } \\
\text { China } \\
\text { Sample Size: } \\
\text { Baseline N: } 90066 \\
\text { Analytic N: } 40510 \\
\text { Attrition: } 55 \% \\
\text { Power Analysis and Sufficient } \\
\text { Sample Size: NR } \\
\text { Sex: } \\
\text { 48\% Female } \\
\text { Race/Ethnicity: } \\
\text { NR } \\
\text { Background Diet: } \\
\text { Ever BF: } 93 \%\end{array}$ & $\begin{array}{l}\text { Intervention/Exposure: } \\
\text { Age of CFB introduction: } \leq 3,4-6,>6 \mathrm{mo} \\
\text { CFB: fish liver oil, rice cereal/porridge, egg yolk, fish paste, liver } \\
\text { paste, tofu, animal blood, bread/steamed bun/fine dried noodle, } \\
\text { ground meat/soy product, and pureed noodle/cookies } \\
\text { Age: } 1,3,6 \text { mo } \\
\text { Assessment Methods: } \\
\text { Maternal interview } \\
\text { Outcomes: } \\
\text { Weight status; Body composition } \\
\text { Age: } 4-5 y \\
\text { Assessment Methods: } \\
\text { Weight status: Obesity defined as BMI z-score>2SD, overweight } \\
\text { defined as between 1-2SD } \\
\text { Body composition: BMl calculated using self-reported weight, height } \\
\text { Confounders accounted for: } \\
\text { Education: } X \\
\text { SES: } X \\
\text { Sex: } X\end{array}$ & $\begin{array}{l}\text { Limitations: } \\
\text { Outcome assessors were not blinded; } \\
\text { Did not use valid/reliable measures to } \\
\text { assess outcomes (weight was self- } \\
\text { reported; lack of detail provided); Did } \\
\text { not account for high loss to follow-up } \\
\text { (55\%); Did not adjust for potential key } \\
\text { confounders (sex, maternal age, } \\
\text { race/ethnicity) } \\
\text { Concern for multiple testing (analyzed } \\
\text { two correlated outcomes [BMI, weight } \\
\text { status] across many types of foods, } \\
\text { increasing chance of false-positives } \\
\text { Cohort data collected over a long } \\
\text { interval (1999-2009) } \\
\text { Limited generalizability of CFB (i.e., } \\
\text { animal blood is not representative of } \\
\text { CFB in the U.S.) and due to population }\end{array}$ \\
\hline
\end{tabular}




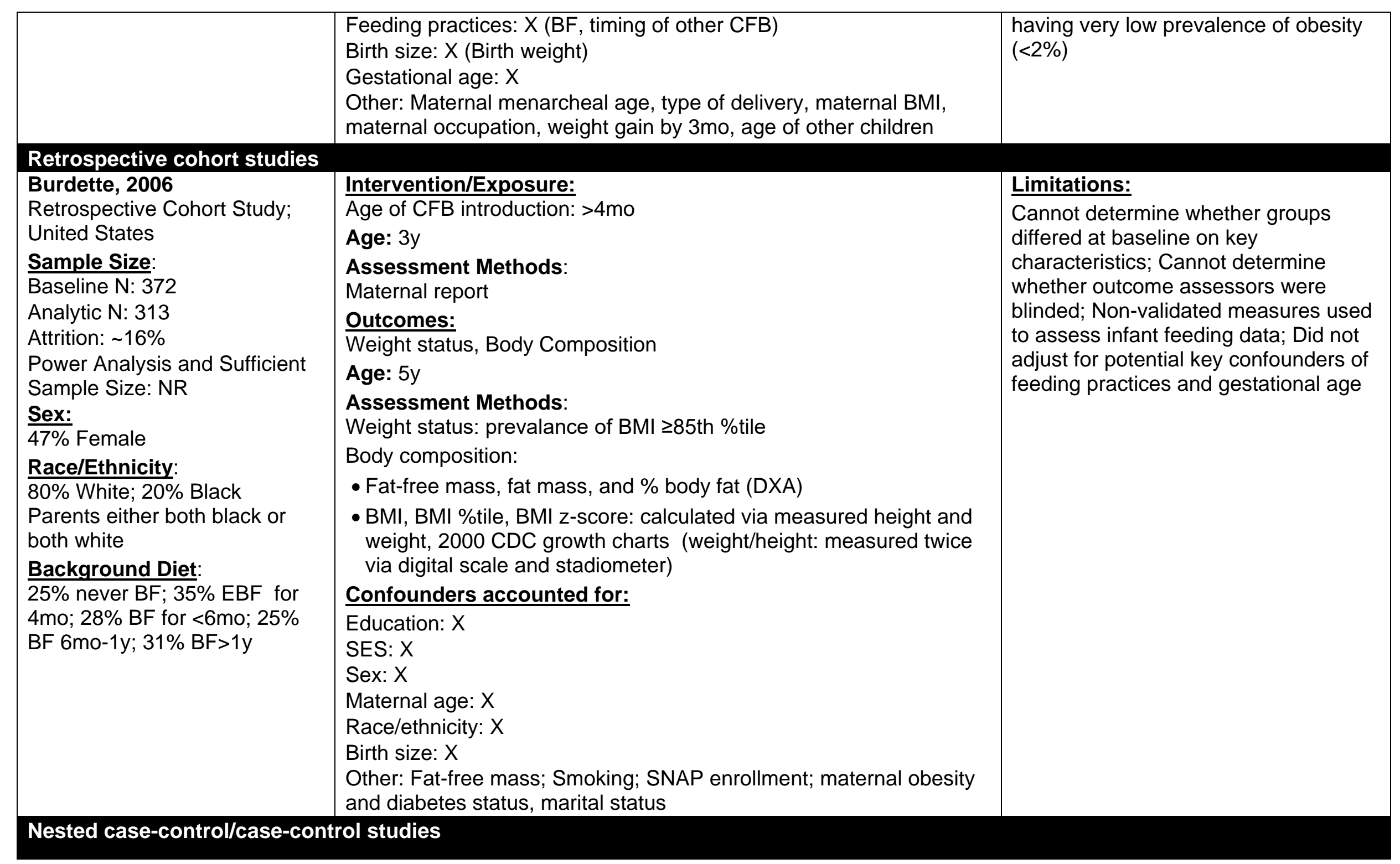




\begin{tabular}{|c|c|c|}
\hline $\begin{array}{l}\text { Bammann, } 2014 \\
\text { Nested Case-Control Study; } 8 \\
\text { European countries: Belgium, } \\
\text { Cyprus, Estonia, Germany, } \\
\text { Hungary, Italy, Spain and } \\
\text { Sweden } \\
\text { Sample Size: } \\
\text { Baseline N: } 1024 \\
\text { Analytic N: } 1024 \\
\text { Attrition: N/A } \\
\text { Power Analysis and Sufficient } \\
\text { Sample Size: Yes; OR 1.6+ } \\
\text { can be detected at } 90 \% \text { power, } \\
\text { two-sided p=0.05 } \\
\text { Sex: } \\
50.3 \% \text { Female } \\
\text { Race/Ethnicity: } \\
\text { NR } \\
\text { Background Diet: } \\
\text { NR }\end{array}$ & $\begin{array}{l}\text { Intervention/Exposure: } \\
\text { Age of CFB introduction:<4mo } \\
\text { CFB: cereals, meat, vegetables or fruits } \\
\text { Age: } 2-9 y \\
\text { Assessment Methods: } \\
\text { Caregiver report } \\
\text { Outcomes: } \\
\text { Weight status (Obesity) } \\
\text { Age: } 2-9 y \\
\text { Assessment Methods: } \\
\text { Weight status: risk of obesity based on IOTF categories; BMI } \\
\text { calculated from measured weight (electronic scale) and length } \\
\text { stadiometer } \\
\text { Confounders accounted for: } \\
\text { Education: } X \\
\text { Sex: } X \\
\text { Feeding practices: } X \\
\text { Birth size: } X \\
\text { Other: Country; Parent BMI; C-section; Smoking; Gestational weight } \\
\text { gain; }\end{array}$ & $\begin{array}{l}\text { Limitations: } \\
\text { Cannot determine whether groups } \\
\text { were similar at baseline on key } \\
\text { characteristics; Cannot determine } \\
\text { whether outcome assessors were } \\
\text { blinded; Cannot determined } \\
\text { validity/reliability of outcome } \\
\text { assessment; Did not adjust for key } \\
\text { confounders (SES, race/ethnicity, } \\
\text { maternal age; gestational age) } \\
\text { Adjusted for maternal BMI which } \\
\text { accounts for maternal age }\end{array}$ \\
\hline $\begin{array}{l}\text { Gungor, } 2010 \\
\text { Case-Control Study; U.S. } \\
\text { Sample Size: } \\
\text { Baseline N: } 129 \\
\text { Analytic N: } 129 \text { (N: } 70 \text { controls } \\
\text { vs. N: } 32 \text { cases; N: } 27 \text { not at } \\
\text { risk) } \\
\text { Attrition: } 0 \% \\
\text { Power Analysis and Sufficient } \\
\text { Sample Size: N/A } \\
\text { Sex: } \\
\text { 54.3\% Female } \\
\text { Race/Ethnicity: }\end{array}$ & $\begin{array}{l}\text { Intervention/Exposure: } \\
\text { Age of CFB introduction: Continuous, mo } \\
\text { Age: } 1 \mathrm{wk}, 1,2,4,6,9,12,15,18,24 \mathrm{mo} \\
\text { Assessment Methods: } \\
\text { Caregiver report via clincal record } \\
\text { Outcomes: } \\
\text { Weight status } \\
\text { Age: } \sim 6.63 \mathrm{y} \text { (SD: } 0.62) \\
\text { Assessment Methods: } \\
\text { Weight, length: measured nude on digital scale to } 0.01 \mathrm{~kg} \text { and } \\
\text { stadiometer to } 0.1 \mathrm{~cm} \text { by trained nurses and recorded } \\
\text { Weight status: overweight defined by age- and sex-specific BMI } \geq \\
\text { 85th percentile at ages } 6,7,8 \mathrm{y} \text { by CDC, } 2000 \text { standards }\end{array}$ & $\begin{array}{l}\text { Limitations: } \\
\text { Cannot determine whether groups } \\
\text { were similar on baseline characteristics } \\
\text { (i.e., other data from baseline } 1 \text { wk- } \\
24 \text { mo NR - maternal age; SES; } \\
\text { race/ethnicity); Cannot determine } \\
\text { reliability/validity of IV/Exposure } \\
\text { (parental feeding data) from clinical } \\
\text { records at ages 1wk, and 1, 2, 4, 6, 9, } \\
12,15,18,24 \text { mo; Weight status } \\
\text { outcomes from recorded weight/height } \\
\text { (measured via trained nurses) between } \\
6-8 y ; \text { Did not adjust for potential key } \\
\text { confounders (maternal age; SES; } \\
\text { race/ethnicity); Other potential }\end{array}$ \\
\hline
\end{tabular}




\begin{tabular}{|c|c|c|}
\hline $\begin{array}{l}\text { Background Diet: } \\
31 \% \text { EBF } \geq 6 \mathrm{mo} \text {; mean BF } \\
\text { duration } 4.57 \mathrm{mo}(\mathrm{SD}: 5.37 \text { ) }\end{array}$ & $\begin{array}{l}\text { Confounders accounted for: } \\
\text { Education: } X \\
\text { Sex: } X \\
\text { Feeding practices: } X \\
\text { Birth size: } X \\
\text { Gestational age: } X \\
\text { Other: Maternal and Paternal education; Parity; Earlier weight gain at } \\
\text { multiple intervals was included in the model from } 0-6 \mathrm{mo} ; 6-12 \mathrm{mo} ; \\
12-18 \mathrm{mo} ; 18-24 \mathrm{mo} ; 0-24 \mathrm{mo}\end{array}$ & $\begin{array}{l}\text { confounders included in analyses as } \\
\text { concurrent exposures showed } \\
\text { differences between resilient vs. } \\
\text { overweight (both parental education; } \\
\text { EBF; earlier weight gain gain intervals } \\
\text { differed bn groups) } \\
\text { Small sample size (N:102 analyzed) }\end{array}$ \\
\hline $\begin{array}{l}\text { Hui, } 2003 \\
\text { Case-Control Study; China } \\
\text { Sample Size: } \\
\text { Baseline N: } 386 \\
\text { Analytic N: } 343 \\
\text { Attrition: } 11 \% \\
\text { Power Analysis and Sufficient } \\
\text { Sample Size: NR } \\
\text { Sex: } \\
52 \% \text { Female } \\
\text { Race/Ethnicity: } \\
\text { NR } \\
\text { Background Diet: } \\
\text { OW: } 71.5 \% \text { never BF; } 10.8 \% \\
\text { ever BF; } 17.7 \% \text { BF } \geq 3 \mathrm{mo} \\
\text { MW: } 70.5 \% \text { never BF; } 18 \% \\
\text { ever BF; } 11.5 \% \text { BF } \geq 3 \mathrm{mo} \\
\text { NW: } 68.5 \% \text { never BF; } 19.2 \% \\
\text { ever BF; } 12.3 \% \text { BF } \geq 3 \mathrm{mo}\end{array}$ & $\begin{array}{l}\text { Intervention/Exposure: } \\
\text { Age of CFB introduction: }<4 \mathrm{mo}(\mathrm{Y} / \mathrm{N}) \\
\text { Age: } 6-7 y \\
\text { Assessment Methods: } \\
\text { Caregiver questionnaire and interview; Diet record, 3d } \\
\text { Outcomes: } \\
\text { Weight status } \\
\text { Age: } 6-7 y \\
\text { Assessment Methods: } \\
\text { Weight status: \% based on derived BMI percentile: overweight group } \\
\left(92^{\text {nd }}\right) \text {, normal middle-weight group }\left(45 \text { th- } 55^{\text {th }}\right) \text {, and normal low- } \\
\text { weight group (<8 }) \\
\text { Confounders accounted for: } \\
\text { Other: parental obesity }\end{array}$ & $\begin{array}{l}\text { Limitations: } \\
\text { Cannot determine whether groups } \\
\text { were similar at baseline; Cannot } \\
\text { determine validity/reliability of outcome } \\
\text { assessment; Did not adjust for } \\
\text { potential key confounders (education, } \\
\text { SES, sex, maternal age, race/ethnicity, } \\
\text { birth size, or gestational age) } \\
\text { Appears they treated IV separately } \\
\text { therefore collinearity is not a concern } \\
\text { but key confounders were not adjusted } \\
\text { for and appeared to differ between } \\
\text { groups }\end{array}$ \\
\hline $\begin{array}{l}\text { Zhou, 2011 } \\
\text { Case-Control Study; China } \\
\text { Sample Size: } \\
\text { Cases: } 81 \\
\text { Controls: } 81 \\
\text { Attrition: N/A }\end{array}$ & $\begin{array}{l}\text { Intervention/Exposure: } \\
\text { Age of CFB introduction: }<4,4-6,>6 \mathrm{mo} \\
\text { Age: } 3-6 y \\
\text { Assessment Methods: } \\
\text { Parent questionnaire }\end{array}$ & $\begin{array}{l}\text { Limitations: } \\
\text { Cannot determine if groups were } \\
\text { similar at baseline on key } \\
\text { characteristics; Did not use } \\
\text { valid/reliable measures to assess } \\
\text { outcomes (growth measures taken }\end{array}$ \\
\hline
\end{tabular}




\begin{tabular}{|l|l|l|}
\hline Power Analysis and Sufficient & Outcomes: & from school records); Did not adjust for \\
Sample Size: NR & Weight status & potential key confounders \\
Sex: & Age: $3-6 y$ & Small sample size; Cannot rule out \\
NR & Assessment Methods: & reverse causality \\
Race/Ethnicity: & Weight status: risk of obese vs. healthy weight based on age- and & sex- specific BMl from IOTF categories, calculated using weight, \\
BR & height from kindergarten records & \\
Background Diet: & Confounders accounted for: & \\
\hline Education: $X$ & SES: $X$ & \\
& Sex: $X$ & Maternal age: $X$ \\
& Feeding practices: $X$ \\
& Birth size: $X$ (Birth weight) \\
& Gestational age: $X$ \\
& Other: Siblings, delivery mode, birth injuries, fetal education, \\
& pregnancy complications, small meal after kindergarten, sleep time, \\
& sports, pickiness, fast $f$ \\
\hline
\end{tabular}

